\title{
Frozen but alive: Molecular responses to autophagy, angiogenesis and energy metabolism in the stress-tolerant wood frog, Rana sylvatica
}

\author{
by \\ Rasha Al-attar
}

B.Sc. University of Ottawa, 2014

M.Sc. Carleton University, 2016

A Thesis Submitted to the Faculty of Graduate Studies and Research in Partial fulfillment of the requirements for the degree of

Doctor of Philosophy

Department of Biology, Specialization in Biochemistry

(C) copyright 2020

Rasha Al-attar 
The undersigned herby recommend to the Faculty of Graduate Studies and Research acceptance of this thesis

Frozen but alive: Molecular responses to autophagy, angiogenesis and energy metabolism in the stress-tolerant wood frog, Rana sylvatica

\author{
Submitted by \\ Rasha Al-attar, B.Sc. M.Sc. \\ in partial fulfillment of the requirements for the degree of Doctor of Philosophy
}

Chair, Department of Biology

Thesis Supervisor

External Examiner

Carleton University 


\begin{abstract}
The freeze-tolerant wood frogs (Rana sylvatica) are incredible creatures that can tolerate the freezing of up to $\sim 70 \%$ of their total body water during winter. Once frozen, these frogs are considered clinically dead, exhibiting no signs of breathing, heartbeat, muscle movement and nerve conductance; yet, they come back to life, unharmed, after a few hours of thawing. Freezing is associated with ischemia due to the freezing of the blood, with hyperglycemia due to the production of large quantities of glucose for cryoprotection, and with dehydration as water moves from inside the cell to the extracellular space to prevent intracellular freezing. Interestingly, wood frogs can tolerate all these stresses independently of freezing, thereby creating a multifactorial model for studying vertebrate freeze-tolerance. Oxygen availability is very low to non-existing during freezing, anoxia, and dehydration; therefore, wood frogs are hypothesized to reduce their overall metabolic rates to balance energy production with energy expenditure in a process called metabolic rate depression (MRD). Animals that undergo MRD reduce energy expensive or detrimental processes and allocate the limited energy available only to pro-survival responses. This thesis examined the effects of freezing and its associated stress on responses to autophagy, angiogenesis, select group of antioxidant enzymes, and energy metabolism. Molecular responses to autophagy demonstrate a significant reduction in autophagosome formation and lysosomal biogenesis in response to anoxia/reoxygenation and to a lesser degree in response to dehydration/rehydration in liver, whereas these two processes were significantly reduced under all conditions in skeletal muscle. Current results also indicate that angiogenesis is regulated in a temporal and stress-dependent manner, where wood frogs increase the expression of certain pro-
\end{abstract}


and anti-angiogenic factors in anticipation of potential damage to capillaries or injury to tissues. Investigation into the role of ETS1 as a transcriptional activator and repressor demonstrated its potential involvement in promoting the expression of select antioxidant enzymes, while repressing the expression of certain nuclear-encoded mitochondrial proteins. Overall, findings in this thesis demonstrate the complexity of the mechanisms involved in controlling metabolic rate depression in adaptive responses in wood frogs. 


\section{Statement of Contribution}

I designed and performed all experiments outlined in this thesis, analyzed the data, generated the figures, and wrote all chapters. Dr. Kenneth B. Storey provided funding, materials and reagents, editorial review, and has approved all chapters. Janet M. Storey assisted in animal sampling and provided editorial review of this thesis.

Chapter 1: Multifaceted regulation of the autophagic machinery in wood frogs Awaiting submission to Biochimica et Biophysica Acta- Gene Regulatory Mechanisms.

Al-attar, R. \& Storey, K.B. (2020). Multifaceted regulation of the autophagic machinery in wood frogs

Chapter 2: RAGE management: ETS1- EGR1 mediated transcriptional networks regulate angiogenesis in wood frogs

Awaiting submission to The International Journal of Biochemistry and Cell Biology.

Al-attar, R. \& Storey, K.B. (2020). RAGE management: ETS1- EGR1 mediated transcriptional networks regulate angiogenesis in wood frogs

Chapter 3: RAGE against the stress: Mitochondrial suppression in hypometabolic hearts This chapter has been published in Gene.

R. Al-Attar, K.B. Storey, RAGE against the stress: Mitochondrial suppression in hypometabolic hearts., $\quad$ Gene. $761 \quad$ (2020) 145039. https://doi.org/10.1016/j.gene.2020.145039.

Permission for inclusion of this manuscript, in part or in full, in an academic thesis or dissertation has been granted by the publisher, Elsevier. 


\section{Acknowledgement}

First and foremost, I would like to give my deepest, most heartfelt thanks to my thesis supervisor, Dr. Kenneth Storey for giving me the opportunity to work under his supervision. Your passion for research, your enthusiasm for teaching, and your leadership in dealing with different people and situations is greatly inspiring. You taught me invaluable lessons both inside and outside the world of science that I will cherish and carry with me for the rest of my life. Your guidance and the opportunities you have given me will no doubt help me excel in my career and put me at a great advantage in my future endeavors. For that, I am forever grateful to you. You are truly one of a kind!

Second, I would like to thank Jan Storey. Jan, you have helped me greatly in the past 6 years, and I could not have done without your support. Thank you for always helping me, being patient with me, and for sending me all those adorable Hudson pictures. You are an incredible human being, and Ken is lucky to have a person like you in his life (and he cannot deny it!).

Third, I would like to thank all the past and present Storey Lab members for all their help and support through my time at the lab. Thank you Hanane for always being there for me, for all the scientific and non-scientific conversations, all the laughter, and for tolerating my NANDOS addiction. Thank you Ranim for being such an awesome friend and TA partner, and for all the entertaining moments. Thank you, Stuart for all the [random] facts, "Rasha's word of the day", and your help with the mousebird stuff. You will be a great entomologist! Thank you, Liam, Alex and Sam, for the awesome 6 years, and I wish you the best in your future careers. The list goes on and on with many more wonderful people, past and present, that made my stay at the Storey Lab a truly great 
experience. I am saddened that I didn't get to say "thank you" to you in person before I leave the lab but blame that on COVID! However, this is not goodbye, and I am anticipating many more outings when I come visit ().

I would like to thank my parents, my aunt, my cousin, and my honorary family member/sister, Tabassom. You were always there for me and pushed me to be the best person I can be. Thank you for everything you have done for me, and I want you to know that without your love and support, I would not be where I am today. Thank you Tabassom for always being there for me, for cheering me through the bad times, for celebrating with me through the good times. I am lucky to have a friend like you.

I would like to extend a special thanks to the amazing wood frog for being so awesome and letting me use its abilities to expand my scientific knowledge. Lastly, thank you to all reviewer $\# 2$ 's out there for always going above and beyond with your very necessary suggestions to make my manuscripts... better... 
Abstract................................................................................................................................................................... III

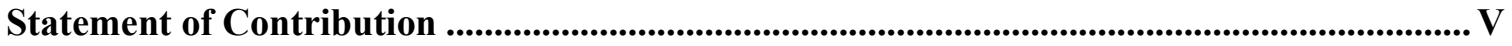

Acknowledgement ...................................................................................................................................VI

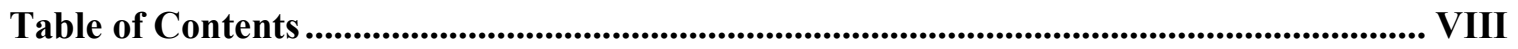

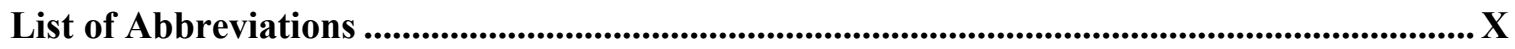

List of Figures............................................................................................................................................. XV

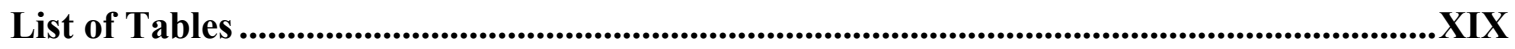

List of Appendices...................................................................................................................................... XX

Chapter 1: General Introduction.............................................................................................................. 1

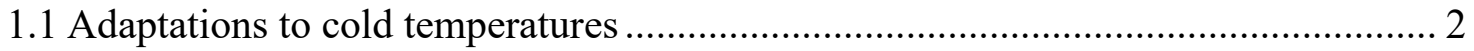

1.2 The freeze-tolerant wood frog, Rana sylvatica .................................................... 3

1.3 Ice nucleation in wood frogs ........................................................................ 4

1.4 Challenges associated with freezing ............................................................. 5

1.5 Adaptations to freezing and thawing in wood frogs ........................................ 6

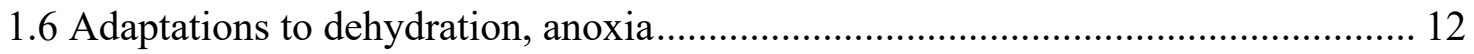

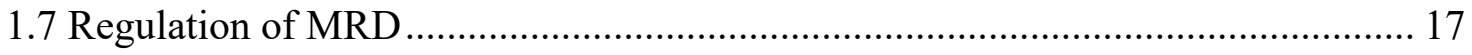

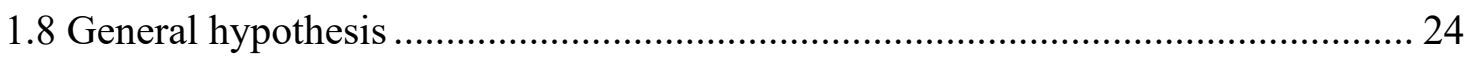

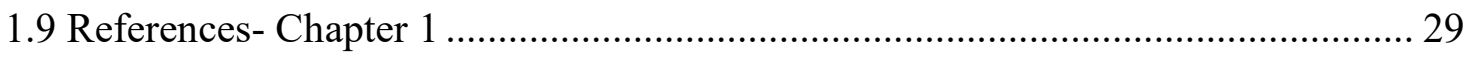

Chapter 2: Multifaceted regulation of the autophagic machinery in wood frogs ...................40

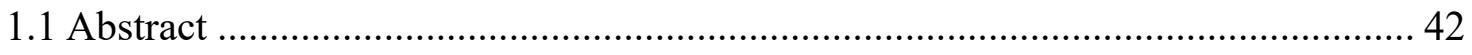

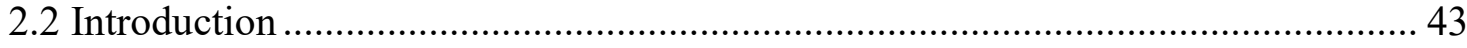

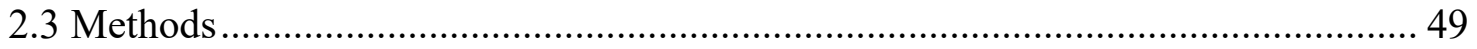

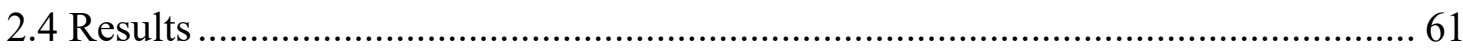

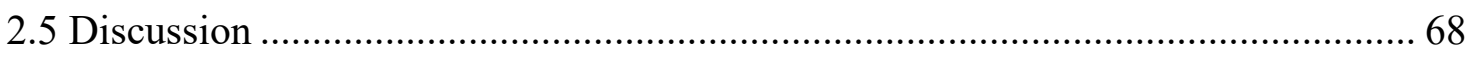

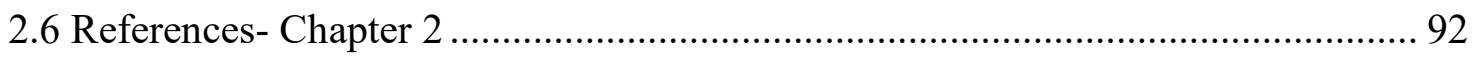

Chapter 3: RAGE management: ETS1- EGR1 mediated transcriptional networks regulate angiogenesis in wood frogs ............................................................................................................117

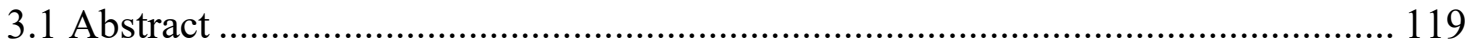

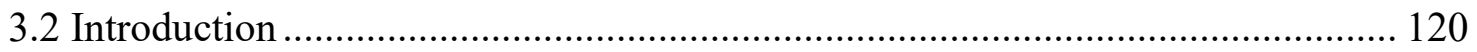

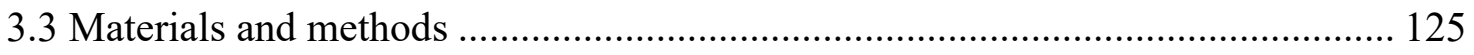

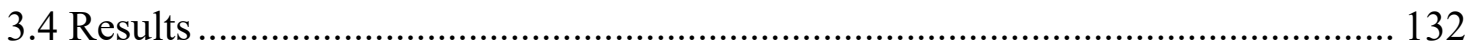

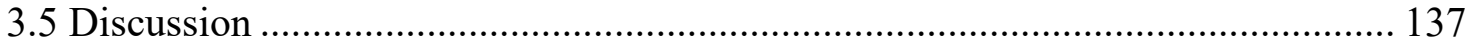

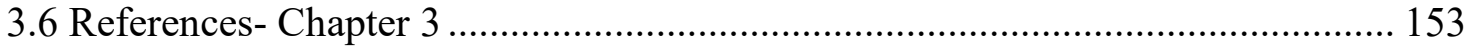


Chapter 4: RAGE against the stress: mitochondrial suppression in hypometabolic hearts 177

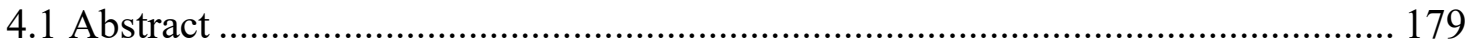

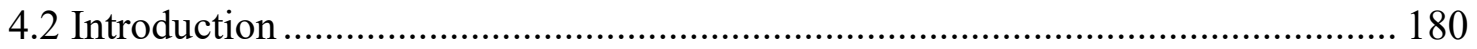

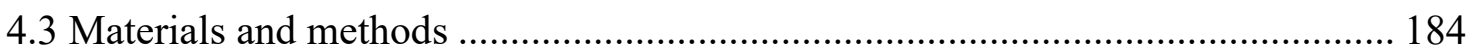

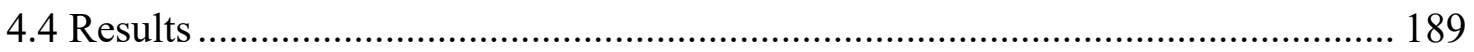

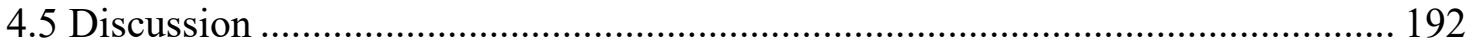

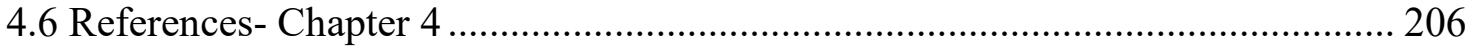

Chapter 5: General discussion ..........................................................................................................222

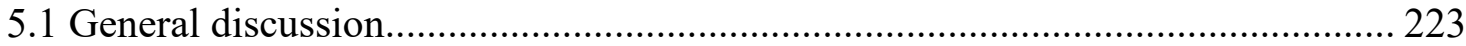

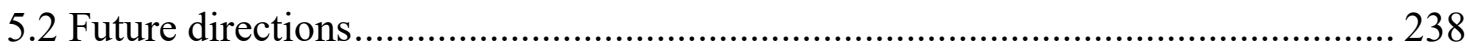

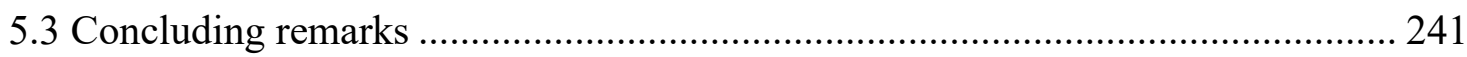

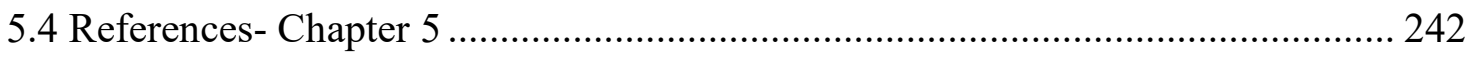


4EBP

ACC

Ac-HMGB1

ADP

AGE

ALEs

AMP

AMPK

ANP

AOX1

ASAH1

ATG

ATP

ATP5A1

ATP6V0D1

BECN1

BGT1

BH3

BSA

CCD

CDS

ChIP-Seq

ChREBP

CLCN7

CLEAR

CML

\section{List of Abbreviations}

Eukaryotic initiation factor 4 binding protein

Acetyl-CoA carboxylase

Acetylated HMGB1

Adenosine diphosphate

Advanced glycation end products

Advanced lipoxidation end products

Adenosine monophosphate

AMP-activated protein kinase

Atrial natriuretic peptide

Aldehyde oxidase 1

Acid ceramidase

Autophagy regulating proteins

Adenosine triphosphate

ATP synthase $\mathrm{H}^{+}$transporting mitochondrial F1 complex, $\alpha$ subunit 1 cardiac muscle

ATPase $\mathrm{H}+$ transporting V0 subunit D1

Beclin1

Betaine-GABA transporters

Bcl-2 homology-3 domain

Bovine serum albumin

Coiled-coil domain

Coding sequence

Chromatin immunoprecipitation with massive parallel DNA sequencing

Carbohydrate-responsive element binding protein

Chloride channel 7

Coordinated lysosomal expression and regulation

Carboxymethyllysine 


\begin{tabular}{|c|c|}
\hline $\mathrm{CS}$ & Citrate synthase \\
\hline CTNS & Cystinosin \\
\hline CTSA & Cathepsin A \\
\hline CYC1 & Cytochrome c1 \\
\hline DMSO & Dimethyl Sulfoxide \\
\hline DNA & Deoxyribonucleic acid \\
\hline dNTP & Deoxynucleoside triphosphate \\
\hline DPI-ELISA & DNA-protein interaction enzyme linked immunosorbent assay \\
\hline DTT & Dithiothreitol \\
\hline ECD & Evolutionarily conserved domain \\
\hline ECL & Enhanced chemiluminescence \\
\hline EDTA & Ethylenediaminetetraacetic acid \\
\hline eEF-2 & Eukaryotic elongation factor 2 \\
\hline eEF-2K & eEF-2 kinase \\
\hline EGF & Endothelial growth factor \\
\hline EGR1 & Early growth response 1 \\
\hline ENCODE & Encyclopedia of DNA elements \\
\hline ER & Endoplasmic reticulum \\
\hline ETS1 & E26 transformation-specific sequence 1 \\
\hline EZH2 & Enhancer of zeste homolog 2 \\
\hline FGF & Fibroblast growth factor \\
\hline FIP200 & FAK family interacting protein 200 \\
\hline GALNS & $\mathrm{N}$-acetylgalactosamine-6-sulfate sulfatase \\
\hline GLUT & Glucose transporter \\
\hline GRP & Glucose-regulated proteins \\
\hline GS & Glycogen synthase \\
\hline GSK3 & Glycogen synthase kinase 3 \\
\hline Gww & Gram wet weight \\
\hline
\end{tabular}




\begin{tabular}{|c|c|}
\hline $\mathrm{HCl}$ & Hydrochloric acid \\
\hline HEPES & 4-(2-hydroxyethyl)-1-piperazineethanesulfonic acid \\
\hline HEXA/B & $\beta$-hexosaminidase \\
\hline HIF $1 \alpha$ & Hypoxia inducible factor $1 \alpha$ \\
\hline HMGB1 & High mobility group box 1 \\
\hline HRP & Horse-radish peroxidase \\
\hline HSP & Heat shock proteins \\
\hline $\mathrm{IgG}$ & Immunoglobulin G \\
\hline JNK1 & c-jun N-terminal kinase 1 \\
\hline $\mathrm{KCl}$ & Potassium chloride \\
\hline $\mathrm{KH}_{2} \mathrm{PO}_{4}$ & Potassium dihydrogen phosphate \\
\hline LC3B & Microtubule-associated proteins light chain 3B \\
\hline LYNUS & Lysosomal nutrient sensing \\
\hline M.W. & Molecular weight \\
\hline MCOLN1 & Mucolipin 1 \\
\hline mDia-1 & Diaphanous-1 \\
\hline MEF2 & Myocyte enhancer factor2 \\
\hline $\mathrm{MgCl}_{2}$ & Magnesium chloride \\
\hline $\mathrm{miR}$ & MicroRNA \\
\hline $\mathrm{MiT} / \mathrm{TFE}$ & Microphthalmia/transcription factor E \\
\hline MRD & Metabolic rate depression \\
\hline mRNA & Messenger RNA \\
\hline mTORC1 & Mechanistic target of rapamycin complex I \\
\hline $\mathrm{Na}_{2} \mathrm{HPO}_{4}$ & Disodium phosphate \\
\hline $\mathrm{Na}_{3} \mathrm{VO}_{4}$ & Sodium orthovanadate \\
\hline NAB1 & NGFI-A binding-1 \\
\hline $\mathrm{NaCl}$ & Sodium chloride \\
\hline $\mathrm{NADH}$ & Nicotinamide adenine dinucleotide \\
\hline
\end{tabular}




\begin{tabular}{|c|c|}
\hline $\mathrm{NaF}$ & Sodium fluoride \\
\hline NAGPA & $\begin{array}{l}\mathrm{N} \text {-acetylglucosamine-1-phosphodiester alpha-N- } \\
\text { acetylglucosaminidase }\end{array}$ \\
\hline NDUFAB1 & NADH dehydrogenase (ubiquinone 1 ) $\alpha / \beta$ subcomplex 1 \\
\hline NFAT5 & Nuclear factor of activated T cell 5 \\
\hline NFATc4 & Nuclear factor of activated T cell 4 \\
\hline NO & Nitric oxide \\
\hline NOS & Nitric oxide synthase \\
\hline NRBF2 & Nuclear receptor binding factor 2 \\
\hline PBS & Phosphate buffer saline \\
\hline $\mathrm{PDH}$ & Pyruvate dehydrogenase complex \\
\hline $\mathrm{PE}$ & Phosphatidylethanolamine \\
\hline Peroxiredoxin & PRX \\
\hline PI3P & Phosphatidylinositol 3-phosphate \\
\hline PKC $\beta I I$ & protein kinase C $\beta I I$ \\
\hline PMSF & Phenylmethylsulfonyl fluoride \\
\hline Pre-microRNA & precursor- microRNA \\
\hline PtdIns $3 K$ & phosphoinositide-3-kinase \\
\hline PTMs & Post-translational modifications \\
\hline PVDF & Polyvinylidiene difluoride \\
\hline RAGE & Receptor for AGE \\
\hline RISC & RNA-induced silencing complex \\
\hline RNA & Ribonucleic acid \\
\hline RNS & Reactive nitrogen species \\
\hline ROS & Reactive oxygen species \\
\hline RT & Room temperature \\
\hline S6K & Ribosomal protein S6 kinase \\
\hline SDS-PAGE & Sodium dodecyl sulphate-polyacrylamide gel electrophoresis \\
\hline SEM & Standard error of mean \\
\hline
\end{tabular}


SMIT

SMURF1

SNARE

SOD

SQSTM1

TBST

TCA

TFEB

TGF

T-HMGB

Tie1/2

TMB

TMEM55B

$\mathrm{TNF} \alpha$

ULK1

UTR

VAMP8

VEGF

VEGFR

VSP
Sodium/myo-inositol cotransporter

SMAD-specific E3 ubiquitin protein ligase 1

SNAP receptor

Superoxide dismutase

Sequestosome 1, p62

Tris buffered saline Tween-20

Tricarboxylic acid cycle

Transcription factor EB

Transforming growth factor

Total HMGB1

Tyrosine kinase with immunoglobulin-like receptors, and EGF-like domain $1 / 2$ receptor

Tetramethylbenzidine

Phosphatidylinositol-4,5-bisphosphate 4-phosphatase

Tumor necrosis factor $\alpha$

Unc-51 like autophagy activating kinase 1

Untranslated region

Vesicle-associated membrane protein 8

Vascular endothelial growth factor

VEGF receptor

Vacuolar sorting protein 


\section{List of Figures}

Figure 1.1: Schematic diagram of microRNA biogenesis. ....................................... 39

Figures 2.1: Schematic diagram showing different phases of autophagy. 104

Figure 2.2: Relative protein levels of key proteins involved in autophagosome formation in liver over the anoxia/reoxygenation cycle measured by immunoblotting. 106

Figure 2.3: Relative protein expression levels of key proteins involved in autophagosome formation in liver over the dehydration/rehydration cycle measured using immunoblotting. 107

Figure 2.4: Relative protein expression levels of key proteins involved in autophagosome formation in skeletal muscle over the anoxia/reoxygenation cycle measured using immunoblotting. 108

Figure 2.5: Relative protein expression levels of key proteins involved in autophagosome formation in skeletal muscle over the dehydration/rehydration cycle measured using immunoblotting.

Figure 2.6: Relative DNA binding activity of TFEB to its consensus sequence in liver and muscle measured by DPI-ELISA. Measurements were done using tissues of wood frogs exposed to A) anoxia/reoxygenation, and B) dehydration/rehydration cycle. ...... 110

Figure 2.7: Relative protein expression levels of TFEB and one of its downstream targets, ATP6V0D1, in liver measured by immunoblotting. Measurements were done using tissues of wood frogs exposed to A) anoxia/reoxygenation, and B) dehydration/rehydration cycle.

Figure 2.8: Relative protein expression levels of TFEB and one of its downstream targets, ATP6V0D1 in muscle measured by immunoblotting. Measurements were done using tissues of wood frogs exposed to A) anoxia/reoxygenation, and B) dehydration/rehydration cycle.

Figure 2.9: Heatmap of relative transcript levels of select autophagy-regulating miRNAs in liver measured using RT-qPCR. Data are presented as heat maps for A) anoxia/reoxygenation and $\mathrm{B}$ ) dehydration/rehydration cycles.

Figure 2.10: Heatmap of relative transcript levels of select autophagy-regulating miRNAs in skeletal muscle measured using RT-qPCR. Data are presented as heat maps for the A) anoxia/reoxygenation and B) dehydration/rehydration cycles. 
Figure 2.11: Relative transcript levels of p62 (sqstm1) in liver of wood frogs measured using RT-qPCR. Measurements were done using liver of wood frogs exposed to A) anoxia/reoxygenation, and B) dehydration/rehydration cycle.

Figure 2.12: Schematic diagram showing the predicted binding sites of mmu-miR-17-5p to different regions of the $p 62$ transcript.

Figure 3.1: Schematic diagram of HMGB1/AGE/RAGE-induced transcriptional responses leading to angiogenic gene transcription. 162

Figure 3.2: Relative protein expression of HMGB1, Ac-HMGB1 (K29) and RAGE measured in liver using immunoblotting. Measurements were done on liver from wood frogs exposed to A) freeze/thaw, B) anoxia/reoxygenation, and C) dehydration/rehydration treatments.

Figure 3.3: Levels of AGE-adducts present in livers measured using the OxiSelect ${ }^{\mathrm{TM}}$ Advanced Glycation End Product (AGE) Competitive ELISA Kit. Measurements were done using liver from wood frogs exposed to A) freeze/thaw, B) anoxia/reoxygenation, C) dehydration/rehydration treatments. 165

Figure 3.4: Relative transcript levels of ets 1 in liver measured by RT-qPCR. Measurements were done in liver of wood frogs expose to A) freeze/thaw, B) anoxia/reoxygenation, C) dehydration/rehydration treatments.

Figure 3.5: Total protein levels of ETS1 and EGR1 in liver measured using immunoblotting. Measurements were done using liver of wood frogs expose to A) freeze/thaw, B) anoxia/reoxygenation, C) dehydration/rehydration treatments. For 169

Figure 3.6: Nuclear localization levels of ETS1, EGR1 and two of EGR1 co-repressors $\mathrm{NAB} 1$ and NAB2 in liver measured using immunoblotting. Measurements were done in liver of wood frogs exposed to A) freeze/thaw, B) anoxia/reoxygenation, C) dehydration/rehydration treatments.

Figure 3.7: Relative DNA binding activity of ETS1 and EGR1 to their consensus sequence in liver measured by DPI-ELISA. Measurements were done using liver of wood frogs expose to A) freeze/thaw, B) anoxia/reoxygenation, C) dehydration/rehydration treatments. 173

Figure 3.8: Relative protein levels of angiogenic factors in liver from wood frogs exposed to freeze/thaw treatments measured by immunoblotting.

Figure 3.9: Relative protein levels of angiogenic factors in liver of wood frogs exposed to anoxia/reoxygenation treatments measured by immunoblotting. 175 
Figure 3.10: Relative protein levels of angiogenic factors in liver of wood frogs expose to dehydration/rehydration treatments measured by immunoblotting. 176

Figure 4.1: Schematic diagram representing physiological and molecular responses that occur during metabolic rate depression in wood frogs. 213

Figure 4.2: Levels of AGE-adducts in wood frog heart in response to freezing, anoxia or dehydration measured using a commercially available assay.

Figure 4.3: Protein levels of upstream RAGE activators and downstream RAGEregulated targets measured using western blotting..... 215

Figure 4.4: Relative nuclear localization of ETS1 proteins in response to freezing, anoxia or dehydration measured by western blotting in wood frog heart. 216

Figure 4.5: Relative DNA binding activity of p-ETS1 (T38) in response to freezing, anoxia or dehydration in wood frog heart.

Figure 4.6: Schematic diagram showing the similarities between the ETS1 monomer generated using the protein sequence of Xenopus tropicalis (NP_001123840_1) and Humans (P14921.1) 218

Figure 4.7: Relative protein expression levels of select ETS1 downstream targets measured by western blotting in wood frog heart.

Figure S4.1: Alignment showing similarities between the ETS1 protein sequence of Xenopus tropicalis (NP_001123840_1) and Humans (P14921.1), generated using Clustal Omega 220

Figure D1: Representative immunoblot showing the A) enhanced chemiluminescent band and B) Coomassie stained membrane of the ATP6V0D1 protein in liver of frogs exposed to control, 24-hour anoxic, and 4-hour reoxygenated treatments. 263

Figure E1: Standard curve generated using AGE-BSA standard following the manufacturer's protocol. 265

Figure F1: Analysis of upstream regulations of NFATc3 measured in wood frog heart in response to freezing, anoxia or dehydration measured by immunoblotting. 267

Figure F2: Relative nuclear levels of NFATc3 (A), and B) the DNA binding activity of total NFATc3 in heart of wood frogs exposed to freezing, anoxia and dehydration measured by immunoblotting. 268

Figure F3: Relative protein levels of downstream targets of NFATc3 and Tfam in wood frog heart exposed to freezing, anoxia and dehydration measured by immunoblotting. 269 
Figure F4: Schematic diagram showing the similarities between the partial NFATc3 protein sequence of Xenopus tropicalis (NP_001135546.1) and splice variant 3 of humans (N P 775186.1). 


\section{List of Tables}

Table 2.1: Nucleotide sequences of miRNA specific forward primers, universal primer and the stem-loop adapter.

Table 2.2: Nucleotide sequences (5'-3') of forward and reverse primers used for mRNA

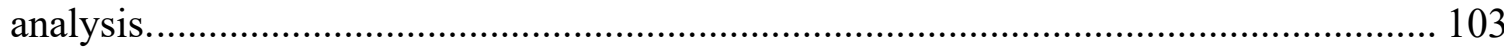

Table S4.1: Shows different posttranslational modifications that could modulate ETS-1 activity.....

Table C1: Immunoblot antibody and protein information. 260 


\section{List of Appendices}

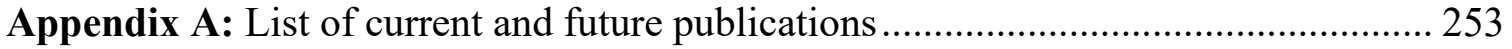

Appendix B: List of Scientific communications at meetings ................................. 257

Appendix C: List of antibodies and immunoblot conditions.................................. 259

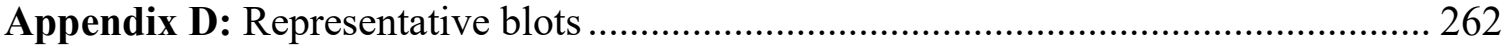

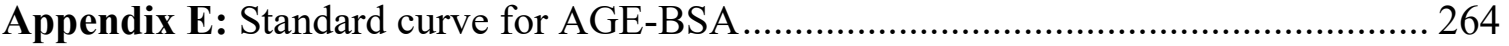

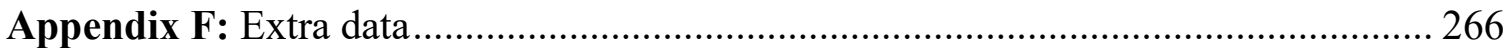




\section{Chapter 1: General Introduction}


Animals living in the wild can be challenged with various unfavorable environmental conditions (e.g. low oxygen levels, scarce food sources, extreme hot or cold temperatures, etc.) that can put their life at risk. Some organisms are highly susceptible to stressful environmental conditions, whereas others have developed sophisticated physiological and biochemical mechanisms that enable them to endure harsh conditions. Understanding the molecular adaptations that stress-tolerant animals use not only increases our knowledge on the plasticity of different biochemical/physiological mechanisms that support life in these conditions, but could also potentially help us solve various medical complications such as diabetes, ischemic stroke and prolonging the storage time of organs prior to transplantation by designing interventions that mimic natural adaptive responses.

\subsection{Adaptations to cold temperatures}

Surviving winter in the wild can be challenging; therefore, adapting a survival strategy becomes imperative for dealing with the deleterious effects of cold temperatures. While different organisms such as some species of birds and butterflies opt for migrating to places with warmer temperatures, others deal with cold temperatures by developing adaptive strategies such as vitrification, anhydrobiosis, freeze-avoidance or simply embracing the cold whether by developing a freeze-tolerance [1]. Vitrification is a survival strategy where the free water available is solidified into an amorphous glass, without forming ice crystals [2]. This process is used by many insect larvae (such as the flat bark beetle) and select species of plants (such as birch trees) to survive winter [2,3]. Anhydrobiosis is another surviving strategy where organisms (such as some prokaryotes and micro fauna) undergo complete dehydration and survive long periods in "suspended 
animation" until favorable conditions arrive $[4,5]$. While anhydrobiosis is not a freezespecific response, a study demonstrated that the Antarctic tardigrades undergoing anhydrobiosis were able to survive and reproduce after $\sim 30$ years of freezing [6]. Freezeavoidance is a winter survival strategy used by many insects, arthropods, and invertebrates to prevent the body fluids from freezing in winter. This is done through the accumulation of high levels of antifreeze proteins, solutes and cryoprotectants (e.g. sorbitol, ribitol, xylitol, erythritol, mannitol, glycerol, glucose, sucrose, or trehalose), which promote the supercooling of the body fluids to temperatures well below the limits that these organisms might have encountered in their natural overwintering habitats [7]. Lastly, freeze-tolerance is an overwintering strategy used by many reptiles, invertebrates, insects, intertidal species, and amphibians $[1,8-11]$. In this category of species, freezing is initiated with a controlled ice nucleation process resulting in the formation of ice crystals on the skin, extracellular and extra-organ spaces, while maintaining a liquid intracellular state.

\subsection{The freeze-tolerant wood frog, Rana sylvatica}

The wood frog, Rana sylvatica, can endure whole body freezing during the winter months. As such, it has become a suitable vertebrate model for studying natural freeze tolerance (and its implications for organ cryopreservation). With a geographic distribution stretching from Alaska, across the boreal forests of Canada and northeastern USA, and down the Appalachian mountain range into the southern USA, these animals endure varying degrees of sub-zero temperatures of $-18{ }^{\circ} \mathrm{C}$ to $-3{ }^{\circ} \mathrm{C}$ for weeks or months at a time $[12,13]$. The large range of geographical distribution of these animals suggests that wood frogs have different degrees of freeze tolerance. Indeed, a study comparing two 
populations of wood frogs demonstrated that frogs native to interior Alaska have a higher degree of freeze-tolerance than frogs native to southern Ohio, as the former population can survive freezing at $-16{ }^{\circ} \mathrm{C}$, which is $\sim 10-13{ }^{\circ} \mathrm{C}$ below the tolerance limit of the Ohioan frogs [14]. Moreover, Alaskan wood frogs were shown to endure 193 consecutive days of freezing in their natural habitat, with $100 \%$ of survival rate upon thawing [15]. This was partly attributed to the fact that Alaskan wood frogs had accumulated higher levels of hepatic glycogen ( $233 \%$ higher during freezing), higher plasma urea $(106 \mu \mathrm{mol} / \mathrm{mL})$, and higher levels of unidentified solutes $(73 \mu \mathrm{mol} / \mathrm{mL})$ compared to Ohioan frogs, which accumulated much lower levels of glycogen, lower uremia $(28 \mu \mathrm{mol} / \mathrm{mL})$ and exhibited no additional unidentified solutes [14].

\subsection{Ice nucleation in wood frogs}

When the temperatures fall in late autumn, wood frogs hide into the subnivean space, under layers of duff and liter on forest floors in hibernacula, that would later prove to be pivotal for their survival. Prolonged periods of physical inactivity (as experienced during freezing) in plain sight renders wood frogs susceptible to predation and extreme dehydration; therefore, hiding under the forest floor is necessary to mitigate these risks. Controlled ice nucleation is an important aspect of freeze-tolerance as it would provide enough time for the wood frog to adjust its physiology and metabolism in preparation for full body freezing. When the moisture in the hibernacula freezes, it seeds the wood frog's skin, and that seeding is necessary to initiate the ice nucleation process that typically occur at $-2.5^{\circ} \mathrm{C}$ to $-3{ }^{\circ} \mathrm{C}$ [8]. Indeed, while $98 \%$ of the wood frogs kept on damp surfaces froze at $-2{ }^{\circ} \mathrm{C}$ without supercooling below the freezing point of their body fluid $\left(-0.5{ }^{\circ} \mathrm{C}\right)$, only $\sim 20 \%$ of these frogs were able to freeze on dry surfaces after they supercooled to - 
$3{ }^{\circ} \mathrm{C}[16,17]$. On dry surfaces, ice nucleation can be triggered by bacterial species (Pseudomonas and Enterobacter species) found on frog's skin or in the gut, a finding that is consistent with the limited supercooling capabilities of these tissues [18]. Amphibian skin is permeable, and this allows ice crystals on the surface of the skin to penetrate into the body and inoculate body fluids, thereby causing internal ice nucleation [17]. Indeed, the movement of ice through the wood frog body was visualized by proton magnetic resonance imaging, and results show that ice moves from outside-in and accumulates in the abdominal cavity, bladder, ventricles of the brain, eye lenses and creates ice sheets between the skin and the skeletal muscle [19].

\subsection{Challenges associated with freezing}

Frozen frogs exhibit no signs of heartbeat, breathing, nerve conductivity or physical movement, but miraculously, recover with no measurable sign of damage soon after thawing [8]. Freezing can be lethal for many reasons. First, formation of ice crystals within the cell can result in architectural damage to the cell membrane and subcellular compartments, the latter resulting in dysfunctional compartmentalization of different cellular processes [20]. Freezing typically occurs in the extracellular space, with ice slowly propagating through the space between organs (between the skin, skeletal muscle, abdominal cavity, etc.), the lumen of the vascular system, and the extracellular fluids that surround the tissues. While intact cell membrane prevents ice penetration into the cell, there are serious challenges that are associated with ice crystal formation in the body. These challenges include but are not limited to [1,2,8,21]: A) potential rupture of capillaries due to ice crystal expansion leading to the loss of vascular integrity and internal bleeding upon thawing, B) potential membrane damage due to extreme 
dehydration as water is withdrawn from the cell to form ice crystals in the extracellular space, resulting in dangerously high cytoplasmic ionic strength C) ischemic damage due to interruption in blood flow, which not only prevents oxygen delivery to organs, but also prevents the cleanup of accumulated waste products, D) metabolic dysfunction as normal oxygen delivery is interrupted, E) oxidative damage due to outburst in reactive oxygen species (ROS) levels soon after thawing and F) potential disuse-induced atrophy of muscles due to prolonged freezing. As such, freeze-tolerant animals must have mechanisms that protects them against the deleterious effects of freezing both at the physiological and molecular levels.

\subsection{Adaptations to freezing and thawing in wood frogs}

Wood frogs have developed physiological, biochemical, and molecular mechanisms that enables their successful survival following freeze-thaw episodes. One of the most important issues that must be addressed in response to freezing is maintaining the architectural integrity of cell and organelle membranes. Indeed, freeze-tolerant invertebrates stabilize the architecture of their cell membranes by incorporating trehalose and prolines, and use large quantities of various cryoprotectants to maintain cellular integrity [1]. Recent studies report the presence of a membrane-associated high molecular mass antifreeze glycolipid (xylomannan) in many freeze-tolerant animals including the Alaskan wood frog, which function to stabilize the membranes in winter [15,22]. Moreover, transcript levels of stearoyl-CoA desaturase (SCD), an enzyme reported to play a significant role in membrane fluidity and repair, were significantly up-regulated in liver of wood frogs during freezing [23,24]. SCD converts palmitoyl-CoA and stearoylCoA to palmitoleate and oleate; two monounsaturated fatty acids that can provide the 
substrates needed for synthesizing different membrane phospholipids [24]. In support of this, a study demonstrated a seasonal change in the liver membrane composition of wood frogs, where winter increased levels of phosphatidylethanolamine to increase membrane fluidity [25]. In addition, organisms that survive long term freezing often have specialized ice-binding proteins that either prevent the recrystallization of smaller ice crystals or minimize their growth into larger crystals, thereby reducing the risk of injuries to tissues or the lumen of the capillaries [8]. For example, wood frogs were reported to express a novel freeze-responsive protein, FR10, which has been recently identified as an ice binding protein. The transcript levels of this protein were significantly induced in liver, brain, lungs, heart, testes, and skeletal muscle in response to freezing, and has been also shown to increase in response to dehydration in heart and brain, and in response to anoxia in the heart, kidneys and lungs [26,27]. Although direct interactions between FR10 and ice crystals has not been shown yet, the 3-dimentional modeling of this protein suggests that it could function as either an ice-binding protein or an anti-freeze protein $[8,26,27]$.

Maintaining extracellular freeze-tolerance and intracellular freeze-avoidance is crucial for wood frog survival, therefore wood frogs and other freeze-tolerant animals use large quantities of cryoprotectants to address this problem. In fact, wood frogs accumulate large reserves of hepatic glycogen in the fall $(180 \mathrm{mg} / \mathrm{g}$ wet weight, $233 \%$ higher in Alaskan frogs) to use as glucose later during freezing $[14,17,28]$. As early as 25 minutes post the initial ice nucleation event on wood frog skin, signals are sent through $\beta$-adrenergic signaling to the liver in efforts to initiate glycogenolysis and generate glucose [29-31]. Glucose levels rise rapidly within minutes, increasing from $5 \mathrm{mM}$ to 
$\sim 40 \mathrm{mM}$ within the first hour post ice nucleation and increasing up to $150-300 \mathrm{mM}$ in core organs in fully frozen animals [28,32]. Simultaneously, within 1 minute of ice nucleation, the heart rate of wood frog doubles to 8 beats/minute to facilitate glucose distribution to different organs [33]. In support of this, wood frogs showed a tissuedependent increase in the numbers of glucose transporters (GLUT) in cell membranes during freezing to enable fast export (from liver) and uptake (by all other organs) of cryoprotectants. For example, it was shown that Glut4 transcripts increased by 2-3 fold in heart to allow glucose uptake, whereas GLUT2 proteins increased significantly in liver to allow for glucose distribution $[32,34]$. The uptake of blood glucose in different tissues is rapid, however it appears that the brain and the core organs have higher concentration of glucose than peripheral tissues such as skeletal muscle and skin, where glucose levels in peripheral tissues rising only to $30-60 \mathrm{mM}$ during freezing [17]. This is potentially due to the inward propagation of ice after ice nucleation, which cuts blood circulation to these tissues, thereby inhibiting glucose delivery to these organs. While glucose is the main cryoprotectant used during freezing, a study on Alaskan wood frogs also reported a modest increase in plasma urea $(106 \mu \mathrm{mol} / \mathrm{mL})$, and additional levels of unidentified solutes $(73 \mu \mathrm{mol} / \mathrm{mL})$ that could be important during freezing [14]. Carbohydrates such as glucose are excellent source of cryoprotectants during freezing for many reasons: a) glucose can be easily synthesized from stored hepatic glycogen, b) it can be easily transported in or out of the cell, c) it can be easily removed from the body when its presence is not needed, d) it has the ability to stabilize proteins and maintain their function during stress, and lastly, e) it is a compatible solute and its accumulation at high levels does not interfere with various enzymatic activities [20]. Although the use of 
glycerol as a cryoprotectant can offer similar benefits without the potential cytotoxicity, glucose is a better choice as its synthesis is more energy efficient [8]. However, high levels of glucose in presence of oxidative stress can result in the formation of highly reactive carbonyl containing compounds that could be conjugated to macromolecules, resulting in various unfavorable outcomes [35]. As such, wood frogs must have developed a regulatory mechanism that would reduce the formation of these conjugates to prevent glucose-induced damage.

Ice crystals exclude solutes from their matrix and given that $65 \%$ of the wood frog's total body water is converted into ice, there is a profound imbalance in the fluid contents between the intracellular and the extracellular compartments. As such, the fluid left in cells and organs will have highly elevated osmolality and ionic strength, that is exacerbated even further after the freeze-induced production of various cryoprotectants [8]. To manage this situation, cells restrict the movements of water as well as small solutes across the plasma membrane and strictly regulate this process via either active transport or facilitated diffusion to maintain an electrochemical gradient that is conducive to each cell's function [36]. The movement of water and small solutes are regulated by the availability and the activity of specific transporters that are either already localized to the surface of the plasma membrane or that are transported to that location via secretory vesicle in response to external stimuli [8]. The availability of these transporters is regulated at multiple biological levels, including during transcription, post-transcription and during translation, whereas their activity is regulated by post-translational modification, localization, and various allosteric inhibitors or activators in response to various internal stimuli [8]. For example, the nuclear factor of activated T cell 5 (NFAT5) 
is an osmoregulatory transcription factor that is sensitive to hypertonicity in different cell types partly by regulating the transporters needed to facilitate the movement of betaine and myo-inositol molecules in or out of the cell [37-40]. NFAT5 regulates the expression of the sodium/myo-inositol cotransporter (SMIT) and the sodium and chloride-dependent betaine-GABA transporters (BGT1) in different cells, and these were shown to be important in regulating the osmotic gradient [37-40]. Previous studies have reported differential regulation of NFAT5 in response to freezing, anoxia and dehydration in multiple tissues of wood frogs $[39,40]$. Interestingly, while protein levels of NFAT5 increased significantly in response to freezing and thawing in wood frog muscle, levels of SMIT remained the same over the course of freezing and thawing, while levels of BGT1 only increased in response to thawing [39]. The same study also demonstrated that despite an increase in NFAT5 levels in liver during freezing, levels of BGT1 and SMIT remained unchanged under the same conditions, there were no significant changes in either protein levels or NFAT5 in response to freezing in the kidney. In response to anoxia, protein levels of NFAT5 were significantly increased which correlated with an increase in BGT1, but not SMIT under the same conditions in the muscle, whereas in liver and kidney there was a significant reduction in NFAT5 levels in response to 24 hour anoxia [40]. Interestingly, the decrease in NFAT5 in the liver had no effect on the protein expression of BGT1 and SMIT in response to anoxia, but levels of BGT1 increased significantly in the kidney under the same condition [40]. On the other hand, responses to dehydration were somewhat different where levels of SMIT in muscle, and BGT1 and SMIT in liver increased significantly despite no change or a decrease in total protein levels of NFAT 5[40]. Altogether, both studies concluded that the expression of these 
transporters is in part regulated at the transcriptional level by NFAT5, and that their expression is tissue and stress dependent in efforts to maintain appropriate intracellular osmolality. While a few of these transporters are studied in wood frogs (GLUTs, as discussed above), future studies are needed to decipher the regulatory mechanisms involved in facilitating or inhibiting the activity of other important transporters in response to stress.

Thawing is another highly regulated process that must be orchestrated in a controlled manner to ensure survival. When temperatures rise in the spring, wood frogs begin to thaw slowly; but unlike freezing, thawing occurs depending on glucose concentrations in tissues. Because high glucose concentration lowers the melting point of the cellular fluids, when temperatures rise, wood frogs thaw from the inside out, with tissues that have higher glucose concentration thawing first [19]. This is advantageous to the frog because it allows the heart, which is necessary to pump blood to all other organs to facilitate thawing, to defrost first. In fact, the first measurable physiological change after thawing is the initiation of cardiac function as early as one hour post thaw, although heart rate is slow and arrhythmic $(\sim 1$ beat/min) at that time point [33]. Moreover, heart rates increased to 13.6 beats/min after 6 hours of thawing as the body temperature of the wood frogs increased to $5^{\circ} \mathrm{C}$, suggesting that perhaps increased cardiac activity plays a crucial role in thawing the remaining organs. Indeed, it was hypothesized that resumption of tissue reperfusion in early hours of thawing and the increase in energy demands in warming organs is potentially the switch needed to change from anaerobic to aerobic metabolism [33]. Following increases in cardiac function and tissue reperfusion, pulmonary breathing, nerve excitability, limb reflexes and normal postures were restored 
within 12 hours post-thawing [13,33]. However, despite a fast-physical recovery from freezing, some physiological and biochemical parameters remain in place for longer periods. For example, given that temperatures in early spring fluctuate, wood frogs retain their high glucose levels for over a week following the initial thawing episode in case another freezing episode occurs [41]. As such, wood frogs must also have regulatory mechanisms in place not only to deal with the ROS generated from reperfusion, but also to protect themselves against glucose-induced damage.

\subsection{Adaptations to dehydration, anoxia}

As discussed earlier, one of the main regulatory mechanisms that enables freezing survival is intracellular dehydration, which not only prevents intracellular ice formation but also provides a sequestration mechanism where ice crystals can form. The skin of many terrestrial amphibians including wood frogs, has very low resistance to water loss under dry or hot environmental conditions; therefore, such amphibians display greater tolerance to dehydration, increased ionic strength and intracellular osmolality compared to other vertebrates [1]. Indeed, it is highly likely that freeze-tolerance stems from preexisting mechanisms that enable animal survival in response to dehydration. For example, when subjecting two freeze-tolerance species of frogs (wood frogs and the spring peeper) to controlled dehydration at $5{ }^{\circ} \mathrm{C}$, both species lost up to $60 \%$ of their total body water and responded with rapid initiation of glycogenolysis in liver and glucose secretion to different organs $[42,43]$. Like thawing, glucose levels in both animals returned to normal after rehydration. It is noteworthy to mention that initiation of hyperglycemic responses during dehydration are not due to water loss by liver cells

directly. Instead, water is initially lost from extra-organ pools without affecting the water 
content of the core organs, unless high percentage of total body water is lost $[42,43]$. This can be seen in wood frogs where despite undergoing $\sim 60 \%$ dehydration of total body water, the water content in liver remained unchanged [43]. Moreover, dehydrating anurans also accumulate high levels of urea and this trend has also been observed in freeze-tolerant animals, albeit to a lower degree $[44,45]$. Urea plays a significant role in increasing the osmolality of body fluids and provides resistant to extreme volume reductions in the cell during dehydration or freezing $[14,46]$.

Wood frogs and other freeze-tolerant animals must also have adaptations to overcome problems associated with anoxia (ischemia) and reoxygenation (reperfusion). This is because after the full onset of freezing, cardiac function comes to a halt and blood freezes, thereby prevent oxygen delivery to organs and creating a state of ischemia. Not surprisingly, wood frogs have been shown to tolerate up to 48 hours of anoxia in chambers filled with $\mathrm{N}_{2}$ gas without exhibiting any sign of damage, independently freezing [47]. Interestingly, exposure to anoxic conditions did not have a significant effect on inducing a hyperglycemic response in wood frogs $[9,48]$. Understandably, a strong hyperglycemic or uremic response is used for cryoprotection purposes during freezing and dehydration where cell volumes are significantly reduced; a response that is absent in anoxic frogs. As such, the limited amount of glucose available is probably used for energy metabolism rather than cryoprotectant purposes. In support of this, a recent phosphoproteomics study of anoxic wood frog liver demonstrated that levels of phosphorylated fructokinase-2 remained unchanged in liver, probably in efforts to promote anaerobic glycolysis until the available glucose is depleted [49]. 
While anoxia tolerance is not extensively studied in the wood frog, research on other anoxia-tolerant models have provided insightful information on the molecular responses needed to survive. Long-term anoxia survival requires several adaptive mechanisms that if absent, could negatively impact survival. These adaptive mechanisms include: A) maintaining a large internal reverse of fermentable fuels such as glycogen, B) supplementing basic glycolytic pathways with other reactions to increase ATP yield per molecule of glucose, C) optimizing the neutralization and acid buffering capacities of the cell, D) managing ATP production with ATP consumption rates, and E) maintaining or inducing a strong antioxidant response system [50]. In most organisms, lipids cannot be used as a source of fuel in anaerobic conditions, therefore such organisms rely on carbohydrate catabolism via glycolysis using glycogen (in animals), starch (plants) or free sugars available in the system to generate energy [50]. Indeed, many anoxia-tolerant vertebrates, including some species of turtles and frogs, build up large glycogen reserves in their liver, which can be converted to glucose and mobilized via the blood to other organs for energy production [51]. Studies on mollusks and other anoxia tolerant organisms also show the use of certain amino acids (e. g. aspartate, asparagine, glutamate, and glutamine) as metabolic fuels under oxygen deprived conditions [50,52]. While using glycolysis to generate energy under anerobic conditions is logical, it has its own limitations: A) low ATP yield per glucose molecule and B) high cellular acidification due to end-product accumulation [50]. As such, some anoxia-tolerant species generate alternative end products (such as opines, ethanol, alanine, propionate, acetate or succinate) to lactate, which not only generate more ATP per glucose molecule catabolized, but also reduce intracellular acidification [50]. In wood frogs, exposure to 
freezing was associated with a significant increase in lactate levels in liver (going from $0.76 \pm 0.11$ in control to $12.1 \pm 1.40 \mu \mathrm{mol} / \mathrm{g}$ wet weight (gww)), heart (going from $6.17 \pm$ $1.00 \mu \mathrm{mol} / \mathrm{gww}$ to $31.0 \pm \mu \mathrm{mol} / \mathrm{gww}$ ), kidney ( going from 8.15 to $35.2 \pm 2.36$ $\mu \mathrm{mol} / \mathrm{gww}$ ) and skeletal muscle (going from $1.99 \pm 0.70$ to $14.8 \pm 1.83 \mu \mathrm{mol} / \mathrm{gww}$ ); suggesting a strong reliance on glycolysis for energy production under this condition [41]. In a species of hypoxia tolerant frogs (Rana temporario), submergence in hypoxic water at $3{ }^{\circ} \mathrm{C}$ resulted in the elevation of plasma lactate levels $(\sim 9 \mathrm{mmol} / \mathrm{L})$, which was returned to baseline $(\sim 1.5 \mathrm{mmol} / \mathrm{L})$ after 8 weeks of treatment [53], suggesting that perhaps alternative regulatory mechanisms exist to buffer lactic acidosis. In anoxic turtles, excessive production of lactate and $\mathrm{H}^{+}$is buffered by the release of $\mathrm{Ca}_{2}^{+}$and $\mathrm{HCO}_{3}^{-}$, which allows for maintaining a safe acid-base homeostatic balance in cells [51].

Due to limited oxygen availability under freezing, anoxia and dehydration, the mitochondrial electron transport chain becomes greatly reduced, thereby shifting cellular metabolism toward anaerobiosis [54]. Despite the presence of large quantities of fermentable fuels under various conditions in wood frogs and adequate buffering systems to prevent intracellular lactic acidosis, using glycolysis to generate energy will not be enough to sustain normal metabolic rates. Under aerobic conditions, one mole of glucose yields 38 moles of ATP; however, under anaerobic conditions, the catabolism of glucose to lactate and $\mathrm{H}^{+}$provides a net of only 2 moles of ATP [55]. The imbalance between energy production and energy consumption is so great that without reprioritizing energy expenditure, wood frogs will not be able to fuel their cells. Hence, these frogs (and many other stress-tolerant species) have developed a coordinated biochemical response that enables them to allocate the finite amount of energy available to fuel pro-survival 
processes while decreasing or completely interrupting all other nonessential or highly energy-expensive processes $[8,51,53,55,56]$. This phenomenon is termed metabolic rate depression (MRD). While metabolic rates have not measured in wood frogs, studies in other animals undergoing similar conditions suggest that without MRD, surviving such conditions is not possible. For example, some species of adult turtles (Chrysemys picta belli) submerged in anoxic water set to $3{ }^{\circ} \mathrm{C}$ reduce their metabolic rates by $\sim 90 \%$ compared to normal conditions, and rely mainly on hepatic glycogen reserve for months [57]. Moreover, frogs that over winter in ice-locked ponds also demonstrate a significant reduction in metabolic rates (down to $\sim 90 \%$ ), as do toads and other species of frogs that live in arid regions of the world [44,58]. Accurate measurement of metabolic rates in frozen animals is difficult because decreasing the body temperature causes a substantial decrease in metabolic rates, and interruption in breathing and cardiac function after the full onset of freezing prevents accurate measurement of oxygen consumption and carbon dioxide release [8]. A study on freeze-tolerant moor frogs (Rana arvalis) showed that oxygen consumption was reduced significantly as body temperature decreased, and this reduction continued until frogs reached a body temperature of $-2{ }^{\circ} \mathrm{C}$, thereafter oxygen consumption remained the same [59]. Moreover, a study on frozen lizards (Lacerta vivipara) showed initiation of freezing resulted in a $40 \%$ decrease in oxygen consumption compared to supercooled animals, and both oxygen consumption and carbon dioxide release fell to zero after $\sim 6$ hours of freezing [60]. These studies show that freezing, anoxia and dehydration (hypoxia-like state)-induced MRD is common amongst stresstolerant animals; suggesting that perhaps wood frogs also reduce their metabolic rates in response to these treatments. This was also partly evident by a study on wood frogs 
where exposure cooling temperatures $\left(4^{\circ} \mathrm{C}\right.$ to $\left.1{ }^{\circ} \mathrm{C}\right)$ showed a significant reduction in carbon dioxide release; suggesting that cold temperatures could potentially reduce metabolic rates [61].

\subsection{Regulation of MRD}

The process of MRD is a complicated network of events that requires synchrony between all cellular processes. Under MRD, the limited energy available is used only to fuel pro-survival processes (e.g. antioxidant response, anti-apoptotic response, etc.) while shutting down all detrimental (e. g. apoptosis), non-essential, or energy expensive processes (e. g. cell cycle, growth, and proliferation) [62-67]. Multiple stress-tolerant organisms decrease their metabolic rates to $1-30 \%$ of their normal levels when faced with unfavorable conditions to conserve the finite amount of energy available [8,51]. By decreasing the metabolic rates, many stress-tolerant animals can stay "inactive" for prolonged periods of time (days, weeks, months or even years) and emerge once environmental conditions are favorable again.

MRD is regulated at various levels of metabolic organization such as at transcriptional, post-transcriptional, translational and post-translational levels [8]. Successful entry into and exist from MRD relies on the synchrony between all regulatory stages; as such, wood frogs must have a coordinated response that enable the induction of few pro-survival processes while reducing or halting the majority of non-essential or detrimental processes. At the transcriptional level, epigenetic modification can alter the accessibility of the DNA to the transcriptional machinery; thereby preventing transcription $[68,69]$. For example, DNA methylation and histone acetylation were shown to play a significant role in regulating hypometabolism in hibernating 13-lined ground 
squirrels and anoxia tolerant freshwater turtles [70,71]. With respect to wood frogs, a reduction in protein levels of histone methyl transferases as well as monomethylated histone (Lys4) proteins were evidence of translational suppression during freezing [68]. The availability of certain transcription factors also contributes to transcriptional regulation in wood frogs. For example, one of the downstream targets of the nuclear factor of activated $\mathrm{T}$ cell 4 (NFATc4) transcription factor is the atrial natriuretic peptide (ANP) gene, which plays a significant role in liver regeneration and repair under stress [72]. As such, NFATc4 DNA binding was elevated in response to anoxia in liver but not skeletal muscle of wood frogs, resulting in the activation of ANP transcription in the former but not the latter tissue [72]. Moreover, the myocyte enhancer factor-2 (MEF2) transcription factor family has been shown to regulate the expression of several genes involved in glucose transport, phosphagen homeostasis and protein quality control check [73]. In wood frogs, total levels of phosphorylated MEF2C remained the same in response to 24 -hour anoxia but not $40 \%$ dehydration in skeletal muscle, a trend that was also similar to MEF2C nuclear localization [73]. Interestingly, transcript expression patterns of glut4, one of the targets regulated by MEF2C that is responsible for glucose uptake, also exhibited no change in response to dehydration but demonstrated a significant increase in response to anoxia in wood frog muscle [73]. The same study also reported no significant change in transcript levels of calreticulin and muscle creatine kinase, suggesting that perhaps their induction is not necessary during dehydration or anoxia survival. Altogether, wood frogs use a combination of DNA modifications and transcription factor availability to regulate transcription under MRD in a temporal and tissue-dependent manner. 
At the post-transcriptional level, wood frogs and other stress-tolerant species have been shown to use microRNA-mediated mechanisms to control the availability and levels of specific transcripts; whereby the increased abundance of specific microRNAs imply translational suppression of the mRNA targets, and reduced microRNA levels imply translation initiation [74,75]. MicroRNAs are short (21-25 nucleotide) single stranded non-coding RNA molecules that negatively regulate the expression of their target genes by silencing the messenger RNA (mRNA) [76]. MicroRNAs are transcribed as primicroRNAs and subsequently cleaved by Drosha to form $\sim 70$ nucleotide precursormicroRNAs (pre-microRNAs) consisting of hairpin structures [76] (Figure 1.2). PremicroRNAs are then exported to the cytoplasm using a RanGTP dependent transporter, Exportin 5 [77]. Once in the cytoplasm, the pre-microRNA is cleaved by Dicer to form short (21-25 nucleotide) single stranded RNAs that will further associate with different proteins to form the RISC structure (RNA-induced silencing complex) and target mRNA via complementarity [76]. The degree of complementary between the microRNA and the target mRNA transcript dictates the fate of that mRNA. High complementarity leads to complete degradation of the transcript, whereas partial complementarity can signal the transcript for storage in p-bodies and stress-granules for future retrieval [78]. In wood frogs, a series of studies have shown differential regulation of microRNAs in a tissue and stress specific manner. For example, miR-16 (microRNA-16) levels increased significantly in liver when wood frogs were exposed to freezing, indicating that translation of downstream transcripts under its control (all components of the cell cycle) was suppressed [79]. Indeed, wood frogs suppress cell cycle activity in response to freezing, anoxia or dehydration stresses using multiple mechanisms including 
microRNA-mediated action [66]. Furthermore, screening of 53 microRNAs in cardiac and skeletal muscle of wood frogs over freeze/thaw showed differential microRNA regulation in a tissue specific manner with 21 microRNAs showing decreased abundance in heart during thawing whereas 16 microRNAs increased during freezing in skeletal muscle [80]. The differentially regulated microRNAs regulated targets involved in arrhythmogenic right ventricular cardiomyopathy, and actin cytoskeleton remodeling [80]. Moreover, differential regulation of microRNAs in response to freezing and thawing in wood frog brain hinted to potential activation of neuroprotective mechanisms such as synaptic signaling and various intracellular signal transduction pathways that lead to protection against ischemia/reperfusion injuries. For example, both exposure to freezing and thawing resulted in the downregulation of miR-204, a microRNA known to negatively impact the expression of the anti-apoptotic BCL2 protein in neuronal cells [81]; suggesting a potential role for miRNAs in regulating apoptosis and promoting survival [82].

Protein synthesis can consume up to $\sim 40 \%$ of the total energy available in the cell. As such, this process is strictly regulated in animals that undergo MRD in efforts to conserve energy. Regulation of protein synthesis occurs mainly by modulating the activity of the translational machinery. This could occurs via polysome disaggregation, which is a hallmark of translational suppression $[50,83]$. Indeed, protein translation rates in liver, kidney and brain was significantly reduced, with levels in the latter tissue decreasing to $0.04 \%$ in response to hibernation (a condition also characterized by MRD) in ground squirrels compared to their euthermic counterparts $[84,85]$. Regulating the activity of the translational machinery can also dictate the rate of protein synthesis. For 
example, studies on liver from frozen wood frogs showed that the AMP-activated protein kinase (AMPK) phosphorylates and activates eEF-2K (eukaryotic elongation factor 2 kinase) which in turn leads to the phosphorylation of eEF-2 (eukaryotic elongation factor 2), thereby inhibiting peptide chain elongation in the frozen state [86]. Moreover, exposure to freezing caused a greater than 50\% decrease in 4EBP (eukaryotic initiation factor 4 binding protein) and S6K (ribosomal protein S6 kinase) total and phosphorylated levels (Jing Zhang, unpublished data). Both 4EBP and S6K play significant roles in controlling mRNA translation and the decrease in both total and active forms of these proteins indicate a significant reduction of mRNA translation during freezing.

At the post-translational level, reversible protein modification (such as phosphorylation, acetylation, ubiquitination, SUMOylation, etc.) has been involved in regulating MRD in several cases $[8,83]$. Post-translational modifications (PTMs) are mediated by various enzymes and the level and activity of these enzymes is subjected to tight regulation in response to various stress conditions. For example, AMPK activity was increased by 2-fold in liver of frozen wood frogs and by 2.5, 4.5 and 3-fold in skeletal muscle of dehydrated, frozen or thawed wood frogs compared to controls [86]. AMPK inhibits multiple biosynthetic enzymes, the best-known being acetyl-CoA carboxylase (ACC), a rate-limiting enzyme of fatty acid biosynthesis, that is inhibited by AMPK phosphorylation at S79. Accordingly, phosphorylated ACC levels rose by 2.5 -folds in liver of frozen wood frogs compared to controls, which lead to the inhibition of fatty acid biosynthesis [86]. Moreover, glycogen synthase kinase 3 (GSK3) was originally known for its involvement in regulating glycogen synthase (GS) activity via phosphorylation and inhibition. Indeed, while total levels of GSK3 did not change in most tissues, 
phosphorylation at S9 decreased significantly during freezing in most wood frog organs, implying that GSK3 was active [87]. Active GSK3 then inhibits GS, and that prevents the re-conversion of glucose back to glycogen under conditions where cryoprotection is still needed [87]. Moreover, the activity of the pyruvate dehydrogenase complex (PDH), a multi-subunit enzyme that links anaerobic glycolysis to the aerobic tricarboxylic acid cycle (TCA), was also shown to be regulated at the post-translational level in wood frog [88]. A study demonstrated an increase in levels of various pyruvate dehydrogenase kinases in response to freezing and but not anoxia in liver of wood frogs [88]. Accordingly, PDH also demonstrated an increase in phosphorylation levels at S293 and S300 in response to freezing but not anoxia; suggesting that this complex is inhibited in response to the former stress [88]. Regulation by PTMs can also facilitate localization and the activity of transcription factors. Indeed, under metabolically depressed states when energy levels are limiting, it is more ideal for animals to stabilize or increase the activity of certain proteins instead of increasing their expression. For example, while the total levels of MEF2C did not change in response to anoxia exposure, it phosphorylated form increased under the same condition, which resulted in its nuclear localization and the induction of select downstream genes [73]. Moreover, the localization of the carbohydrate-responsive element binding protein (ChREBP) transcription factor was hypothesized to be regulated by PTMs in response to freezing in a temporal manner [23]. Phosphorylation can also act to inhibit the activity of select transcription factors. For example, NFATc4's activity is inhibited by phosphorylation; and an increase in its phosphatase (calcineurin A) was demonstrated in response to anoxia exposure in wood frog liver [72]. This increase resulted in NFATc4 dephosphorylation, NFATc4 activation 
and its subsequent nuclear translocation, resulting in the induction of its pro-survival downstream genes in anoxic wood frogs [72]. Altogether, reversible PTMs play a significant role in regulating MRD by modulating the activity, stability, and localization of various proteins.

Although each regulatory stage is significant on its own, MRD requires a coordinated response at all regulatory levels. For example, while inhibitory mechanisms at the epigenetic level may be enough to prevent transcription by denying DNA accessibility to the transcriptional machinery, decreased activity (controlled by posttranslational modifications) of specific transcription factors or co-regulators could also be involved. Likewise, even if total protein levels of specific targets remain unchanged, an increase in various post-translational modifications could modulate their activity by either causing temporary inhibition or by stabilizing the available proteins to increase their halflife. However, even under conditions with $\sim 80-95 \%$ overall suppression in metabolism, stress-tolerant organisms manage to increase the activity a small set of pro-survival pathways by strategically channeling the available energy to those processes. For example, whereas the cell cycle is halted, the anti-apoptotic response is induced to help protect cells. A study on wood frogs exposed to anoxic conditions showed the overexpression of anti-apoptotic proteins, Bcl-xL and c-IAP in the liver [64]. Moreover, ROS production is a major hallmark of hypoxic/ischemic events and can cause even more problems upon reoxygenation/reperfusion. Furthermore, hyperglycemia and increased protein glycation are also known to promote prooxidant environments in the cell. Therefore, wood frogs and other stress-tolerant animals increase their antioxidant defense system accordingly. Indeed, wood frogs were confirmed to have higher basal levels of 
antioxidant defenses compared to leopard frogs [8,62]. For example, glutathione peroxidase activity rose by 2.5 -fold in various tissues, and was also accompanied by an increase in glutathione levels during freezing $[8,62]$. Moreover, levels of select antioxidant enzymes such as the cytosolic superoxide dismutase1 (SOD1) and the mitochondrial SOD2 were upregulated in brain and heart of wood frogs respectively in response to freezing [65]. Additionally, protein levels of catalase was also upregulated in response to anoxia in brain, suggesting that this antioxidant enzymes is required for ROS detoxification in this tissue [65]. Wood frogs also differentially regulate the protein levels of various heat shock proteins (HSPs) and glucose-regulated proteins (GRPs) in response to stress. Indeed, protein levels of HSP110 was significantly elevated in response to freezing in liver and kidney, in response to anoxia treatment in liver, brain, and heart, and in response to dehydration treatments in muscle, brain heart and skin of wood frogs [63]. Protein levels of HSP70 also showed differential regulation, with levels rising in liver, muscle, heart and kidney in response to freezing, in muscle in response to anoxia, and in muscle, heart and kidney in response to dehydration [63]. Differential regulation of HSP60, HSP10, GRP78, and GRP94 were also reported in a tissue and stress-dependent manner [63]. Altogether, it is clear that despite strict energy utilization under MRD, wood frogs strategically and selectively induce the expression of select antioxidant and prosurvival factors to mitigate potential damage that could be cause in early stages, prolonged exposure or early phases of recovery from different stresses.

\subsection{General hypothesis: Molecular response to freezing, anoxia and dehydration require the induction of various genes involved in regulating autophagy, angiogenesis, antioxidant response and energy metabolism in a temporal, tissue, and stress-dependent manner in anticipation for potential damage.}


Objective 1: Multifaceted regulation of the autophagic machinery in wood frogs

Autophagy is a complex process, characterized by multiple stages (initiation, nucleation, elongation, and fusion) involving the formation of an autophagosome, a vesicle-like structure that engulfs defective cytoplasmic molecules, before fusing with the lysosome to facilitate cargo degradation $[89,90]$. Autophagy has been extensively studied in multiple animal models and in response to many environmental and disease conditions, as dysregulation in autophagy could result in cell death [89,91,92]. Given its importance in maintaining cellular homeostasis, it was of interest to see how autophagy is regulated in response to dehydration/rehydration and anoxia/reoxygenation treatments in wood frog liver and skeletal muscle. Briefly, regulation of autophagy involves the activity of several transcription factors, chiefly Transcription factor EB (TFEB), which promotes the transcription of numerous genes involved in autophagosome formation and lysosomal biogenesis $[93,94]$. Autophagy is also regulated at the post-transcriptional level by microRNAs, which target specific components of the autophagosome machinery, thereby promoting or inhibiting their translation $[95,96]$. Moreover, autophagy can also be regulated at the post-translational level by PTMs [97]. Indeed, series of PTMs (phosphorylation, glycosylation, acetylation, methylation, lipidation, etc.) have been demonstrated to be crucial for either inducing or inhibiting autophagy in response to various stimuli [97,98]. In Chapter 2, regulation of autophagy will be studied at multiple biological levels to decipher the dominant mode of regulation under each stress and tissue in wood frogs. Logically, wood frogs have regulatory mechanisms that are induced early after the initial exposure to stress in anticipation of potential damage that could result after the full onset of stress or in early hours of recovery. While these regulatory 
mechanisms may not be required at first, their availability on standby mode may be crucial for mitigating potential damage when it arises. However, autophagy is an energy expensive process, therefore it is likely that wood frogs reduce autophagic flux during stress.

Specific hypothesis 1: Autophagy is an energy expensive process, therefore autophagosome formation and lysosomal biogenesis will be attenuated in response to stress, and this process is regulated at multiple biological levels in a tissue and stress dependent manner.

Objective 2: RAGE management: ETS1- EGR1 mediated transcriptional networks regulate angiogenesis in wood frogs

Freezing, thawing, dehydration, and or rehydration can cause potential rupture of capillaries, leading to the loss of vascular integrity. Moreover, these stresses could also result in tissue damage that if not dealt with appropriately, could result in organ failure. As such, one of the protective mechanisms that could be induced in anticipation of these events is angiogenesis; the formation of new blood vessels rooting from pre-existing parent vessels [99-101]. Induction of angiogenesis in response to ischemia in the heart or in response to tissue injury has been demonstrated to improve cardiac function and accelerate wound healing $[102,103]$. Angiogenesis, like many other processes in the cell is regulated at multiple biological levels. At the transcriptional level, pro and antiangiogenic targets are regulated by many transcription factors chiefly the early growth response 1 (EGR1) and the E26 transformation-specific sequence 1 (ETS-1) transcript 
factors [99-101]. Upstream to these two factors is the activation of the receptor for advanced glycation end product (RAGE), which results in phosphorylation of several kinases that lead to the induction and activation of EGR1 and ETS1 in response to stress $[104,105]$. Freezing and dehydration are hyperglycemic events, and the presence of high levels of glucose in the cell concurrently with the presence of oxidative stress accelerates the formation of advanced glycation end products (AGEs) [35]. AGEs can associate with various macromolecules, and negatively impact their function, stability, and clearance. An earlier study on wood frogs demonstrated that glycated serum albumin is elevated 7 days post-thawing, thereby providing the first line of evidence that AGE formation could be occurring in wood frogs. On the other hand, exposure to oxidative stress causes the release of the nuclear high mobility group box 1 (HMGB1) protein to the cytoplasm and subsequently extracellular space [106,107]. Both AGEs and HMGB1 are ligands of RAGE and could potentially result in the activation of this receptor and subsequent induction of EGR1 and ETS1 transcription factors. In Chapter 3, activation of HMGB1/AGE/RAGE in response to freezing/thawing, anoxia/reoxygenation and dehydration/rehydration in wood frog liver will be investigated. Moreover, this chapter will focus on EGR1 and ETS1- regulated targets that are involved in angiogenesis to determine whether this process occurs in wood frogs in response to stress.

Specific hypothesis 2: Pro and anti-angiogenic factors will be induced in response to stress in wood frog liver, however their expression will be regulated in a temporal manner in preparation for potential repair of damaged capillaries or wound healing. 


\section{Objective 3: RAGE-mediated transcriptional responses in wood frog heart}

As mentioned in objective 2, freezing and dehydration are hyperglycemic events that could result in the formation of AGEs, HMGB1 release from the nucleus to the cytoplasm, and RAGE activation. A previous cDNA array screen in wood frog heart demonstrated an increase in RAGE levels in response to freezing [32]. RAGE-mediated signaling results in the activation of ETS1, a transcription factor that regulates a wide range of downstream genes involved in numerous processes [108-112]. Other than angiogenesis (discussed in objective 2), ETS1 also regulates genes encoding antioxidant enzymes, glucose transport and metabolism, and nuclear encoded mitochondrial proteins [110-113]. Interestingly, ETS1 can also act as a transcriptional repressor to select group of downstream genes $[114,115]$. Studies have shown that overexpression of ETS1 results in transcriptional inhibition of select nuclear encoded mitochondrial genes such as the NADH dehydrogenase (ubiquinone 1) $\alpha / \beta$ subcomplex 1 (NDUFAB1), cytochrome c1 (CYC1), and ATP synthase $\mathrm{H}^{+}$transporting mitochondrial $\mathrm{F} 1$ complex, $\alpha$ subunit 1 cardiac muscle (ATP5A1) [111,112]. This chapter will examine whether AGE/HMGB1/RAGE mediated activation of ETS1 occurs in the heart of wood frogs exposed to freezing, anoxia and dehydration, and whether this activation will result in induction or repression of select downstream genes.

Specific hypothesis 3: ETS1 acts both as a transcriptional activator and a transcriptional repressor. Specifically, ETS1 will inhibit the transcription of select nuclear encoded mitochondrial genes, while inducing the expression of select antioxidant enzymes in response to stress. 


\subsection{References- Chapter 1}

[1] K.B. Storey, J.M. Storey, Natural freeze survival in animals, Annu. Rev. Ecol. Syst. 27 (1996) 365-386. https://doi.org/10.1146/annurev.ecolsys.27.1.365.

[2] K.B. Storey, J.M. Storey, Physiology, biochemistry and molecular biology of vertebrate freeze tolerance: The wood frog, in: B.J. Fuller, N. Lane, E.E. Benson (Eds.), Life a Frozen State, CRC Press, Boca Raton, 2004: pp. 243-274.

[3] T. Sformo, K. Walters, K. Jeannet, B. Wowk, G.M. Fahy, B.M. Barnes, J.G. Duman, Deep supercooling, vitrification and limited survival to $-100 \mathrm{C}$ in the Alaskan beetle Cucujus clavipes puniceus (Coleoptera: Cucujidae) larvae, J. Exp. Biol. 213 (2010) 502-509. https://doi.org/10.1242/jeb.035758.

[4] M. Potts, Desiccation tolerance of prokaryotes., Microbiol. Rev. 58 (1994) 755805. https://doi.org/10.1128/MMBR.58.4.755-805.1994.

[5] D.A. Wharton, Water loss and morphological changes during desiccation of the anhydrobiotic nematode Ditylenchus dipsaci, J. Exp. Biol. 199 (1996) 1085-1093.

[6] M. Tsujimoto, S. Imura, H. Kanda, Recovery and reproduction of an Antarctic tardigrade retrieved from a moss sample frozen for over 30 years, Cryobiology. 72 (2016) 78-81. https://doi.org/10.1016/j.cryobiol.2015.12.003.

[7] K.B. Storey, J.M. Storey, Insect cold hardiness: Metabolic, gene, and protein adaptation, Can. J. Zool. 90 (2012) 456-475. https://doi.org/10.1139/z2012-011.

[8] K.B. Storey, J.M. Storey, Molecular physiology of freeze tolerance in vertebrates, Physiol. Rev. 97 (2017) 623-665. https://doi.org/10.1152/physrev.00016.2016.

[9] K.B. Storey, J.M. Storey, Natural freeze tolerance in ectothermic vertebrates., Annu. Rev. Physiol. 54 (1992) 619-37. https://doi.org/10.1146/annurev.ph.54.030192.003155.

[10] K.B. Storey, Reptile freeze tolerance: Metabolism and gene expression, Cryobiology. 52 (2006) 1-16. https://doi.org/10.1016/j.cryobiol.2005.09.005.

[11] K.B. Storey, J.M. Storey, Biochemistry of freeze tolerance in terrestrial insects, Trends Biochem. Sci. 8 (1983) 242-245. https://doi.org/10.1016/09680004(83)90349-3.

[12] L.B. Middle, B.M. Barnes, Overwintering physiology of the wood frog, Rana sylvatica, in interior Alaksa, Am. Zool. 41 (2001) 1526-1527.

[13] J.P. Costanzo, R.E. Lee, Avoidance and tolerance of freezing in ectothermic vertebrates, J. Exp. Biol. 216 (2013) 1961-1967. https://doi.org/10.1242/jeb.070268.

[14] J.P. Costanzo, M.C.F. do Amaral, A.J. Rosendale, R.E. Lee, Hibernation physiology, freezing adaptation and extreme freeze tolerance in a northern population of the wood frog., J. Exp. Biol. (2013).

https://doi.org/10.1242/jeb.089342. 
[15] D.J. Larson, L. Middle, H. Vu, W. Zhang, A.S. Serianni, J. Duman, B.M. Barnes, Wood frog adaptations to overwintering in Alaska: New limits to freezing tolerance, J. Exp. Biol. (2014). https://doi.org/10.1242/jeb.101931.

[16] R.E. Lee, J.P. Costanzo, Biological ice nucleation and ice distribution in coldhardy ectotermic animals., Annu. Rev. Physiol. 60 (1998) 55-72. https://doi.org/10.1146/annurev.physiol.60.1.55.

[17] K.B. Storey, J.M. Storey, Freeze tolerance in animals, Physiol. Rev. 68 (1988) 2784. https://doi.org/10.1152/physrev.1988.68.1.27.

[18] M.R. Lee, R.E. Lee, J.M. Strong-Gunderson, S.R. Minges, Isolation of icenucleating active bacteria from the freeze-tolerant frog, Rana sylvatica, Cryobiology. 32 (1995) 358-365. https://doi.org/10.1006/cryo.1995.1036.

[19] B. Rubinsky, S.T. Wong, J.S. Hong, J. Gilbert, M. Roos, K.B. Storey, 1H magnetic resonance imaging of freezing and thawing in freeze-tolerant frogs., Am. J. Physiol. 266 (1994) R1771-7. https://doi.org/10.1152/ajpregu.1994.266.6.R1771.

[20] J.M. Storey, K.B. Storey, Cold hardiness and freeze tolerance, in: Funct. Metab., John Wiley \& Sons, Inc., Hoboken, NJ, USA, 2005: pp. 473-503. https://doi.org/10.1002/047167558X.ch17.

[21] K.B. Storey, J.M. Storey, Molecular biology of freezing tolerance, Compr. Physiol. 3 (2013) 1283-1308. https://doi.org/10.1002/cphy.c130007.

[22] K.R. Walters, A.S. Serianni, Y. Voituron, T. Sformo, B.M. Barnes, J.G. Duman, A thermal hysteresis-producing xylomannan glycolipid antifreeze associated with cold tolerance is found in diverse taxa, J. Comp. Physiol. B. 181 (2011) 631-640. https://doi.org/10.1007/s00360-011-0552-8.

[23] R. Al-attar, C.-W. Wu, K.K. Biggar, K. Storey, Carb-loading: Freeze-induced activation of the glucose-responsive ChREBP transcriptional network in wood frogs, Physiol. Biochem. Zool. (2019) 706463. https://doi.org/10.1086/706463.

[24] M. Miyazaki, J.M. Ntambi, Role of stearoyl-coenzyme A desaturase in lipid metabolism, Prostaglandins, Leukot. Essent. Fat. Acids. 68 (2003) 113-121. https://doi.org/10.1016/S0952-3278(02)00261-2.

[25] A.M. Reynolds, R.E. Lee, J.P. Costanzo, Membrane adaptation in phospholipids and cholesterol in the widely distributed, freeze-tolerant wood frog, Rana sylvatica, J. Comp. Physiol. B. 184 (2014) 371-383. https://doi.org/10.1007/s00360-014-0805-4.

[26] Q. Cai, K.B. Storey, Upregulation of a novel gene by freezing exposure in the freeze-tolerant wood frog (Rana sylvatica), Gene. 198 (1997) 305-312. https://doi.org/10.1016/S0378-1119(97)00332-6.

[27] K.J. Sullivan, K.K. Biggar, K.B. Storey, Transcript expression of the freeze responsive gene fr10 in Rana sylvatica during freezing, anoxia, dehydration, and development, Mol. Cell. Biochem. 399 (2015) 17-25. 
https://doi.org/10.1007/s11010-014-2226-9.

[28] K.B. Storey, J.M. Storey, Freeze tolerant frogs: Cryoprotectants and tissue metabolism during freeze-thaw cycles, Can. J. Zool. 64 (1986) 49-56. https://doi.org/10.1139/z86-008.

[29] S.J. Hemmings, K.B. Storey, Alterations in hepatic adrenergic receptor status in Rana sylvatica in response to freezing and thawing: Implications to the freezeinduced glycemic response., Can. J. Physiol. Pharmacol. 72 (1994) 1552-60. http://www.ncbi.nlm.nih.gov/pubmed/7736347.

[30] J.M. Storey, K.B. Storey, Triggering of cryoprotectant synthesis by the initiation of ice nucleation in the freeze tolerant frog, Rana sylvatica, J. Comp. Physiol. B. 156 (1985) 191-195. https://doi.org/10.1007/BF00695773.

[31] K.B. Storey, Glycolysis and the regulation of cryoprotectant synthesis in liver of the freeze tolerant wood frog, J. Comp. Physiol. B. 157 (1987) 373-380. https://doi.org/10.1007/BF00693364.

[32] K.B. Storey, Strategies for exploration of freeze responsive gene expression: Advances in vertebrate freeze tolerance, Cryobiology. 48 (2004) 134-145. https://doi.org/10.1016/j.cryobiol.2003.10.008.

[33] J.R. Layne, R.E. Lee, T.L. Heil, Freezing-induced changes in the heart rate of wood frogs (Rana sylvatica)., Am. J. Physiol. 257 (1989) R1046-9. https://doi.org/10.1152/ajpregu.1989.257.5.R1046.

[34] A.J. Rosendale, B.N. Philip, R.E. Lee, J.P. Costanzo, Cloning, characterization, and expression of glucose transporter 2 in the freeze-tolerant wood frog, Rana sylvatica, Biochim. Biophys. Acta. 1840 (2014) 1701-1711. https://doi.org/10.1016/j.bbagen.2013.12.011.

[35] T.J. Lyons, A.J. Jenkins, Glycation, oxidation, and lipoxidation in the development of the complications of diabetes: A carbonyl stress hypothesis., Diabetes Rev. (Alexandria, Va.). 5 (1997) 365-391.

[36] L. Reuss, Water transport across cell membranes, in: ELS, John Wiley \& Sons, Ltd, Chichester, UK, 2012. https://doi.org/10.1002/9780470015902.a0020621.pub2.

[37] Z. Zhang, J.D. Ferraris, H.L. Brooks, I. Brisc, M.B. Burg, Expression of osmotic stress-related genes in tissues of normal and hyposmotic rats, Am. J. Physiol. Physiol. 285 (2003) F688-F693. https://doi.org/10.1152/ajprenal.00028.2003.

[38] S. Maallem, A. Berod, M. Mutin, H.M. Kwon, M.L. Tappaz, Large discrepancies in cellular distribution of the tonicity-induced expression of osmoprotective genes and their regulatory transcription factor TonEBP in rat brain, Neuroscience. 142 (2006) 355-368. https://doi.org/10.1016/j.neuroscience.2006.06.028.

[39] Y. Zhang, R. Al-attar, K.B. Storey, TonEBP/NFAT5 regulates downstream osmoregulatory proteins during freeze-thaw stress in the wood frog, Cryobiology. 79 (2017) 43-49. https://doi.org/10.1016/j.cryobiol.2017.09.003. 
[40] R. Al-attar, Y. Zhang, K.B. Storey, Osmolyte regulation by TonEBP/NFAT5 during anoxia-recovery and dehydration-rehydration stresses in the freeze-tolerant wood frog (Rana sylvatica), PeerJ. 5 (2017) e2797. https://doi.org/10.7717/peerj.2797.

[41] K.B. Storey, J.M. Storey, Biochemical adaption for freezing tolerance in the wood frog, Rana sylvatica, J. Comp. Physiol. B. 155 (1984) 29-36. https://doi.org/10.1007/BF00688788.

[42] T.A. Churchill, K.B. Storey, Effects of dehydration on organ metabolism in the frog Pseudacris crucifer : Hyperglycemic responses to dehydration mimic freezinginduced cryoprotectant production, J. Comp. Physiol. B. 164 (1994) 492-498. https://doi.org/10.1007/BF00714587.

[43] T.A. Churchill, K.B. Storey, Dehydration tolerance in wood frogs: A new perspective on development of amphibian freeze tolerance, Am. J. Physiol. Integr. Comp. Physiol. 265 (1993) R1324-R1332. https://doi.org/10.1152/ajpregu.1993.265.6.R1324.

[44] K.B. Storey, Life in the slow lane: Molecular mechanisms of estivation., Comp. Biochem. Physiol. A. Mol. Integr. Physiol. 133 (2002) 733-54. https://doi.org/10.1016/S1095-6433(02)00206-4.

[45] J.P. Costanzo, R.E. Lee, Cryoprotection by urea in a terrestrially hibernating frog, J. Exp. Biol. 208 (2005) 4079-4089. https://doi.org/10.1242/jeb.01859.

[46] J.P. Costanzo, R.E. Lee, Urea loading enhances freezing survival and postfreeze recovery in a terrestrially hibernating frog, J. Exp. Biol. 211 (2008) 2969-2975. https://doi.org/10.1242/jeb.019695.

[47] C.P. Holden, K.B. Storey, Second messenger and cAMP-dependent protein kinase responses to dehydration and anoxia stresses in frogs., J. Comp. Physiol. B. 167 (1997) 305-12. https://doi.org/10.1007/s003600050078.

[48] K.B. Storey, J.M. Storey, Signal transduction and gene expression in the regulation of natural freezing survival, in: Cell Mol. Response to Stress, 2001: pp. 1-19. https://doi.org/10.1016/S1568-1254(01)80003-6.

[49] L.J. Hawkins, M. Wang, B. Zhang, Q. Xiao, H. Wang, K.B. Storey, Glucose and urea metabolic enzymes are differentially phosphorylated during freezing, anoxia, and dehydration exposures in a freeze tolerant frog, Comp. Biochem. Physiol. Part D Genomics Proteomics. 30 (2019) 1-13. https://doi.org/10.1016/j.cbd.2019.01.009.

[50] K.B. Storey, J.M. Storey, Oxygen limitation and metabolic rate depression, in: Funct. Metab., John Wiley \& Sons, Inc., Hoboken, NJ, USA, 2005: pp. 415-442. https://doi.org/10.1002/047167558X.ch15.

[51] D.C. Jackson, G.R. Ultsch, Physiology of hibernation under the ice by turtles and frogs, J. Exp. Zool. Part A Ecol. Genet. Physiol. 313 A (2010) 311-327.

https://doi.org/10.1002/jez.603. 
[52] G. Gäde, W.R. Ellington, The anaerobic molluscan heart: Adaptation to environmental anoxia. Comparison with energy metabolism in vertebrate hearts, Comp. Biochem. Physiol. Part A Physiol. 76 (1983) 615-620. https://doi.org/10.1016/0300-9629(83)90463-2.

[53] P.H. Donohoe, R.G. Boutilier, The protective effects of metabolic rate depression in hypoxic cold submerged frogs, Respir. Physiol. 111 (1998) 325-336. https://doi.org/10.1016/S0034-5687(97)00125-4.

[54] A. Krivoruchko, K.B. Storey, Forever young: Mechanisms of natural anoxia tolerance and potential links to longevity., Oxid. Med. Cell. Longev. 3 (2010) 186198. https://doi.org/10.4161/oxim.3.3.4.

[55] K.B. Storey, Molecular mechanisms of anoxia tolerance, Int. Congr. Ser. 1275 (2004) 47-54. https://doi.org/10.1016/j.ics.2004.08.072.

[56] A. Gupta, K.B. Storey, Regulation of antioxidant systems in response to anoxia and reoxygenation in Rana sylvatica, Comp. Biochem. Physiol. Part B Biochem. Mol. Biol. 243-244 (2020) 110436. https://doi.org/10.1016/j.cbpb.2020.110436.

[57] C. V. Herbert, D.C. Jackson, Temperature effects on the responses to prolonged submergence in the turtle Chrysemys picta bellii. II. Metabolic rate, blood acidbase and ionic changes, and cardiovascular function in aerated and anoxic water, Physiol. Zool. 58 (1985) 670-681. https://doi.org/10.1086/physzool.58.6.30156071.

[58] P.H. Donohoe, T.G. West, R.G. Boutilier, Respiratory, metabolic, and acid-base correlates of aerobic metabolic rate reduction in overwintering frogs, Am. J. Physiol. Integr. Comp. Physiol. 274 (1998) R704-R710. https://doi.org/10.1152/ajpregu.1998.274.3.R704.

[59] Y. Voituron, L. Paaschburg, M. Holmstrup, H. Barré, H. Ramløv, Survival and metabolism of Rana arvalis during freezing, J. Comp. Physiol. B. 179 (2009) $223-$ 230. https://doi.org/10.1007/s00360-008-0307-3.

[60] Y. Voituron, B. Verdier, C. Grenot, The respiratory metabolism of a lizard (Lacerta vivipara) in supercooled and frozen states, Am. J. Physiol. Integr. Comp. Physiol. 283 (2002) R181-R186. https://doi.org/10.1152/ajpregu.00378.2001.

[61] B.J. Sinclair, J.R. Stinziano, C.M. Williams, H.A. Macmillan, K.E. Marshall, K.B. Storey, Real-time measurement of metabolic rate during freezing and thawing of the wood frog, Rana sylvatica: Implications for overwinter energy use, J. Exp. Biol. 216 (2013) 292-302. https://doi.org/10.1242/jeb.076331.

[62] D.R. Joanisse, K.B. Storey, Oxidative damage and antioxidants in Rana sylvatica, the freeze-tolerant wood frog., Am. J. Physiol. 271 (1996) R545-53. https://doi.org/10.1152/ajpregu.1996.271.3.R545.

[63] J.M. Storey, K.B. Storey, In defense of proteins: Chaperones respond to freezing, anoxia, or dehydration stress in tissues of freeze tolerant wood frogs, J. Exp. Zool. Part A Ecol. Integr. Physiol. 331 (2019) 392-402. https://doi.org/10.1002/jez.2306. 
[64] V.E.M. Gerber, S. Wijenayake, K.B. Storey, Anti-apoptotic response during anoxia and recovery in a freeze-tolerant wood frog (Rana sylvatica)., PeerJ. 4 (2016) e1834. https://doi.org/10.7717/peerj.1834.

[65] C.-W. Wu, S.N. Tessier, K.B. Storey, Stress-induced antioxidant defense and protein chaperone response in the freeze-tolerant wood frog Rana sylvatica, Cell Stress Chaperones. 23 (2018) 1205-1217. https://doi.org/10.1007/s12192-0180926-x.

[66] J. Zhang, K.B. Storey, Cell cycle regulation in the freeze-tolerant wood frog, Rana sylvatica, Cell Cycle. 11 (2012) 1727-1742. https://doi.org/10.4161/cc.19880.

[67] R. Roufayel, K.K. Biggar, K.B. Storey, Regulation of cell cycle components during exposure to anoxia or dehydration stress in the wood frog, Rana sylvatica, J. Exp. Zool. Part A Ecol. Genet. Physiol. 315A (2011) 487-494. https://doi.org/10.1002/jez.696.

[68] L.J. Hawkins, K.B. Storey, Histone methylation in the freeze-tolerant wood frog (Rana sylvatica), J. Comp. Physiol. B. 188 (2018) 113-125. https://doi.org/10.1007/s00360-017-1112-7.

[69] K.B. Storey, Regulation of hypometabolism: Insights into epigenetic controls., J. Exp. Biol. 218 (2015) 150-9. https://doi.org/10.1242/jeb.106369.

[70] S. Wijenayake, K.B. Storey, The role of DNA methylation during anoxia tolerance in a freshwater turtle (Trachemys scripta elegans ), J. Comp. Physiol. B Biochem. Syst. Environ. Physiol. 186 (2016) 333-342. https://doi.org/10.1007/s00360-0160960-x.

[71] P.J. Morin, K.B. Storey, Evidence for a reduced transcriptional state during hibernation in ground squirrels, Cryobiology. 53 (2006) 310-318. https://doi.org/10.1016/j.cryobiol.2006.08.002.

[72] R. Al-Attar, K.B. Storey, Effects of anoxic exposure on the nuclear factor of activated T cell (NFAT) transcription factors in the stress-tolerant wood frog., Cell Biochem. Funct. 36 (2018) 420-430. https://doi.org/10.1002/cbf.3362.

[73] M.P. Hoyeck, H. Hadj-Moussa, K.B. Storey, The role of MEF2 transcription factors in dehydration and anoxia survival in Rana sylvatica skeletal muscle, PeerJ. 5 (2017) e4014. https://doi.org/10.7717/peerj.4014.

[74] K.K. Biggar, K.B. Storey, The emerging roles of microRNAs in the molecular responses of metabolic rate depression, J. Mol. Cell Biol. 3 (2011) 167-175. https://doi.org/10.1093/jmcb/mjq045.

[75] K.K. Biggar, K.B. Storey, Functional impact of microRNA regulation in models of extreme stress adaptation, J. Mol. Cell Biol. 10 (2018) 93-101. https://doi.org/10.1093/jmcb/mjx053.

[76] L. He, G.J. Hannon, Correction: MicroRNAs: Small RNAs with a big role in gene regulation, Nat. Rev. Genet. 5 (2004) 631-631. https://doi.org/10.1038/nrg1415. 
[77] A. Grishok, A.E. Pasquinelli, D. Conte, N. Li, S. Parrish, I. Ha, D.L. Baillie, A. Fire, G. Ruvkun, C.C. Mello, Genes and mechanisms related to RNA interference regulate expression of the small temporal RNAs that control C. elegans developmental timing, Cell. 106 (2001) 23-34.

[78] J. Liu, M.A. Valencia-Sanchez, G.J. Hannon, R. Parker, MicroRNA-dependent localization of targeted mRNAs to mammalian P-bodies, Nat. Cell Biol. 7 (2005) 719-723. https://doi.org/10.1038/ncb1274.

[79] K.K. Biggar, A. Dubuc, K. Storey, MicroRNA regulation below zero: Differential expression of miRNA-21 and miRNA-16 during freezing in wood frogs, Cryobiology. 59 (2009) 317-321. https://doi.org/10.1016/j.cryobiol.2009.08.009.

[80] S. Bansal, B.E. Luu, K.B. Storey, MicroRNA regulation in heart and skeletal muscle over the freeze-thaw cycle in the freeze tolerant wood frog, J. Comp. Physiol. B. 186 (2016) 229-241. https://doi.org/10.1007/s00360-015-0951-3.

[81] A. Sacconi, F. Biagioni, V. Canu, F. Mori, A. Di Benedetto, L. Lorenzon, C. Ercolani, S. Di Agostino, A.M. Cambria, S. Germoni, G. Grasso, R. Blandino, V. Panebianco, V. Ziparo, O. Federici, P. Muti, S. Strano, F. Carboni, M. Mottolese, M. Diodoro, E. Pescarmona, A. Garofalo, G. Blandino, miR-204 targets Bcl-2 expression and enhances responsiveness of gastric cancer, Cell Death Dis. 3 (2012) e423-e423. https://doi.org/10.1038/cddis.2012.160.

[82] H. Hadj-Moussa, K.B. Storey, Micromanaging freeze tolerance: The biogenesis and regulation of neuroprotective microRNAs in frozen brains, Cell. Mol. Life Sci. 75 (2018) 3635-3647. https://doi.org/10.1007/s00018-018-2821-0.

[83] R. Al-Attar, K.B. Storey, Suspended in time: Molecular responses to hibernation also promote longevity., Exp. Gerontol. 134 (2020) 110889. https://doi.org/10.1016/j.exger.2020.110889.

[84] J.E. Knight, E.N. Narus, S.L. Martin, A. Jacobson, B.M. Barnes, B.B. Boyer, mRNA stability and polysome loss in hibernating arctic ground squirrels (Spermophilus parryii), Mol. Cell. Biol. 20 (2000) 6374-6379. https://doi.org/10.1128/MCB.20.17.6374-6379.2000.

[85] K.U. Frerichs, C.B. Smith, M. Brenner, D.J. DeGracia, G.S. Krause, L. Marrone, T.E. Dever, J.M. Hallenbeck, Suppression of protein synthesis in brain during hibernation involves inhibition of protein initiation and elongation., Proc. Natl. Acad. Sci. U. S. A. 95 (1998) 14511-6. https://doi.org/10.1073/pnas.95.24.14511.

[86] M.H. Rider, N. Hussain, S. Horman, S.M. Dilworth, K.B. Storey, Stress-induced activation of the AMP-activated protein kinase in the freeze-tolerant frog Rana sylvatica, Cryobiology. 53 (2006) 297-309. https://doi.org/10.1016/j.cryobiol.2006.08.001.

[87] C.A. Dieni, M.C. Bouffard, K.B. Storey, Glycogen synthase kinase-3: Cryoprotection and glycogen metabolism in the freeze-tolerant wood frog., J. Exp. Biol. 215 (2012) 543-51. https://doi.org/10.1242/jeb.065961. 
[88] R. Al-Attar, S. Wijenayake, K.B. Storey, Metabolic reorganization in winter: Regulation of pyruvate dehydrogenase (PDH) during long-term freezing and anoxia., Cryobiology. 86 (2019) 10-18. https://doi.org/10.1016/j.cryobiol.2019.01.006.

[89] N. Mizushima, M. Komatsu, Autophagy: Renovation of cells and tissues, Cell. 147 (2011) 728-741. https://doi.org/10.1016/j.cell.2011.10.026.

[90] C. He, D.J. Klionsky, Regulation mechanisms and signaling pathways of autophagy, Annu. Rev. Genet. 43 (2009) 67-93. https://doi.org/10.1146/annurevgenet-102808-114910.

[91] N. Mizushima, B. Levine, A.M. Cuervo, D.J. Klionsky, Autophagy fights disease through cellular self-digestion, Nature. 451 (2008) 1069-1075. https://doi.org/10.1038/nature06639.

[92] H.S. Jung, K.W. Chung, J. Won Kim, J. Kim, M. Komatsu, K. Tanaka, Y.H. Nguyen, T.M. Kang, K.H. Yoon, J.W. Kim, Y.T. Jeong, M.S. Han, M.K. Lee, K.W. Kim, J. Shin, M.S. Lee, Loss of autophagy diminishes pancreatic $\beta$ cell mass and function with resultant hyperglycemia, Cell Metab. 8 (2008) 318-324. https://doi.org/10.1016/j.cmet.2008.08.013.

[93] M. Palmieri, S. Impey, H. Kang, A. di Ronza, C. Pelz, M. Sardiello, A. Ballabio, Characterization of the CLEAR network reveals an integrated control of cellular clearance pathways, Hum. Mol. Genet. 20 (2011) 3852-3866. https://doi.org/10.1093/hmg/ddr306.

[94] M. Sardiello, M. Palmieri, A. di Ronza, D.L. Medina, M. Valenza, V.A. Gennarino, C. Di Malta, F. Donaudy, V. Embrione, R.S. Polishchuk, S. Banfi, G. Parenti, E. Cattaneo, A. Ballabio, A Gene Network Regulating Lysosomal Biogenesis and Function, Science (80-. ). (2009). https://doi.org/10.1126/science.1174447.

[95] L.B. Frankel, A.H. Lund, MicroRNA regulation of autophagy, Carcinogenesis. 33 (2012) 2018-2025. https://doi.org/10.1093/carcin/bgs266.

[96] H. Li, D. Miao, Q. Zhu, J. Huang, G. Lu, W. Xu, MicroRNA-17-5p contributes to osteoarthritis progression by binding p62/SQSTM1., Exp. Ther. Med. 15 (2018) 1789-1794. https://doi.org/10.3892/etm.2017.5622.

[97] Y. Xie, R. Kang, X. Sun, M. Zhong, J. Huang, D.J. Klionsky, D. Tang, Posttranslational modification of autophagy-related proteins in macroautophagy, Autophagy. 11 (2015) 28-45. https://doi.org/10.4161/15548627.2014.984267.

[98] Y.L. Deribe, T. Pawson, I. Dikic, Post-translational modifications in signal integration, Nat. Struct. Mol. Biol. 17 (2010) 666-672.

https://doi.org/10.1038/nsmb.1842.

[99] A.M. Randi, A. Sperone, N.H. Dryden, G.M. Birdsey, Regulation of angiogenesis by ETS transcription factors., Biochem. Soc. Trans. 37 (2009) 1248-53.

https://doi.org/10.1042/BST0371248. 
[100] N.A. Abdel-Malak, M. Mofarrahi, D. Mayaki, L.M. Khachigian, S.N.A. Hussain, Early growth response-1 regulates angiopoietin-1-induced endothelial cell proliferation, migration, and differentiation, Arterioscler. Thromb. Vasc. Biol. 29 (2009) 209-216. https://doi.org/10.1161/ATVBAHA.108.181073.

[101] R.G. Fahmy, C.R. Dass, L.-Q. Sun, C.N. Chesterman, L.M. Khachigian, Transcription factor Egr-1 supports FGF-dependent angiogenesis during neovascularization and tumor growth, Nat. Med. 9 (2003) 1026-1032. https://doi.org/10.1038/nm905.

[102] P. Kumar, S. Kumar, E.P. Udupa, U. Kumar, P. Rao, T. Honnegowda, Role of angiogenesis and angiogenic factors in acute and chronic wound healing, Plast. Aesthetic Res. 2 (2015) 243. https://doi.org/10.4103/2347-9264.165438.

[103] T. Johnson, L. Zhao, G. Manuel, H. Taylor, D. Liu, Approaches to therapeutic angiogenesis for ischemic heart disease, J. Mol. Med. 97 (2019) 141-151. https://doi.org/10.1007/s00109-018-1729-3.

[104] Y. Xu, F. Toure, W. Qu, L. Lin, F. Song, X. Shen, R. Rosario, J. Garcia, A.M. Schmidt, S.F. Yan, Advanced glycation end product (AGE)-receptor for age (RAGE) signaling and up-regulation of Egr-1 in hypoxic macrophages, J. Biol. Chem. 285 (2010) 23233-23240. https://doi.org/10.1074/jbc.M110.117457.

[105] J.S. Chang, T. Wendt, W. Qu, L. Kong, Y.S. Zou, A.M. Schmidt, S.F. Yan, Oxygen deprivation triggers upregulation of early growth response-1 by the receptor for advanced glycation end products, Circ. Res. 102 (2008) 905-913. https://doi.org/10.1161/CIRCRESAHA.107.165308.

[106] S. Gardella, C. Andrei, D. Ferrera, L. V. Lotti, M.R. Torrisi, M.E. Bianchi, A. Rubartelli, The nuclear protein HMGB1 is secreted by monocytes via a nonclassical, vesicle-mediated secretory pathway, EMBO Rep. 3 (2002) 995-1001. https://doi.org/10.1093/embo-reports/kvf198.

[107] J. Evankovich, S.W. Cho, R. Zhang, J. Cardinal, R. Dhupar, L. Zhang, J.R. Klune, J. Zlotnicki, T. Billiar, A. Tsung, High mobility group box 1 release from hepatocytes during ischemia and reperfusion injury is mediated by decreased histone deacetylase activity., J. Biol. Chem. 285 (2010) 39888-97. https://doi.org/10.1074/jbc.M110.128348.

[108] Z. Gao, G.H. Kim, A.C. Mackinnon, A.E. Flagg, B. Bassett, J.U. Earley, E.C. Svensson, Ets 1 is required for proper migration and differentiation of the cardiac neural crest., Development. 137 (2010) 1543-51. https://doi.org/10.1242/dev.047696.

[109] H. Pei, C. Li, Y. Adereth, T. Hsu, D.K. Watson, R. Li, Caspase-1 is a direct target gene of ETS1 and plays a role in ETS1-induced apoptosis, Cancer Res. 65 (2005) 7205-7213. https://doi.org/10.1158/0008-5472.CAN-04-3566.

[110] M. Shiota, H. Izumi, N. Miyamoto, T. Onitsuka, E. Kashiwagi, A. Kidani, G. Hirano, M. Takahashi, M. Ono, M. Kuwano, S. Naito, Y. Sasaguri, K. Kohno, Ets regulates peroxiredoxin 1 and 5 expressions through their interaction with the high- 
mobility group protein B1, Cancer Sci. 99 (2008) 1950-1959.

https://doi.org/10.1111/j.1349-7006.2008.00912.x.

[111] M.L. Verschoor, L.A. Wilson, C.P. Verschoor, G. Singh, Ets-1 Regulates Energy Metabolism in Cancer Cells, PLoS One. 5 (2010) e13565. https://doi.org/10.1371/journal.pone.0013565.

[112] M.L. Verschoor, C.P. Verschoor, G. Singh, Ets-1 global gene expression profile reveals associations with metabolism and oxidative stress in ovarian and breast cancers, Cancer Metab. 1 (2013) 17. https://doi.org/10.1186/2049-3002-1-17.

[113] X. Zhang, D. Wu, M. Aldarouish, X. Yin, C. Li, C. Wang, ETS-1: A potential target of glycolysis for metabolic therapy by regulating glucose metabolism in pancreatic cancer, Int. J. Oncol. 50 (2017) 232-240. https://doi.org/10.3892/ijo.2016.3770.

[114] G. Mavrothalassitis, J. Ghysdael, Proteins of the ETS family with transcriptional repressor activity, Oncogene. 19 (2000) 6524-6532. https://doi.org/10.1038/sj.onc.1204045.

[115] D. Goldman, M.K. Sapru, S. Stewart, J. Plotkin, T.A. Libermann, B. Wasylyk, K. Guan, Cloning and characterization of GETS-1, a goldfish Ets family member that functions as a transcriptional repressor in muscle, Biochem. J. 335 (1998) 267275. https://doi.org/10.1042/bj3350267.

[116] K.K. Biggar, K.B. Storey, Insight into post-transcriptional gene regulation: Stressresponsive microRNAs and their role in the environmental stress survival of tolerant animals, J. Exp. Biol. 218 (2015) 1281-1289.

https://doi.org/10.1242/jeb.104828. 


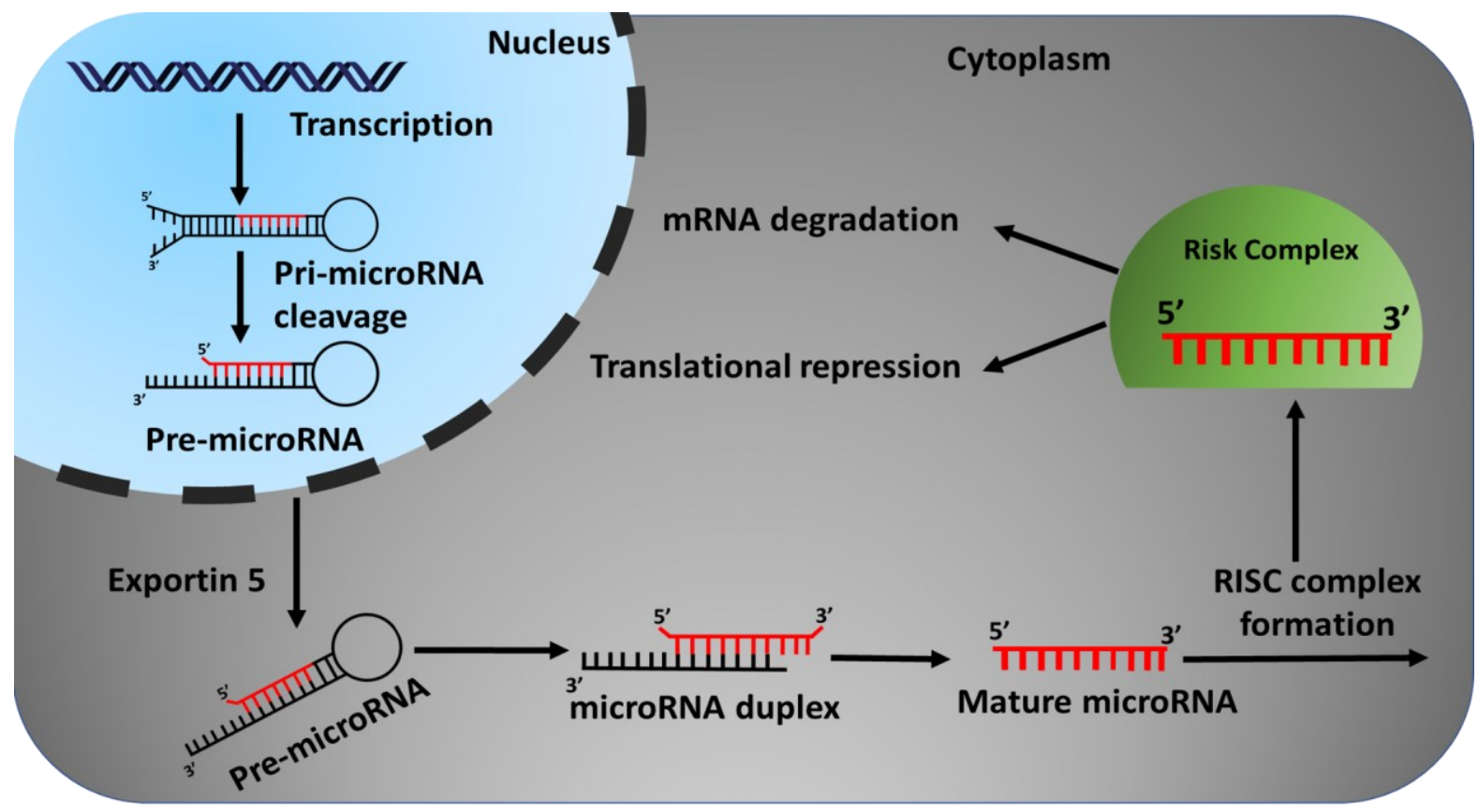

Figure 1.1: Schematic diagram of microRNA biogenesis. 


\section{Chapter 2: Multifaceted regulation of the autophagic machinery in wood frogs}




\section{Multifaceted regulation of the autophagic machinery in wood frogs}

Rasha Al-attar ${ }^{1}$, Kenneth B. Storey ${ }^{1}$

${ }^{1}$ Institude of Biochemistry and Department of Biology, Carleton University, Ottawa, ON, Canada (K1S 5B6)

Corresponding Author:

Kenneth B. Storey

Kenstorey@cunet.carleton.ca

Telephone: +1 (613) 520-2600 x3678 


\subsection{Abstract}

The wood frog, Rana sylvatica, is one of the freeze-tolerant vertebrates known to date. After the full onset of freezing, the wood frog is clinically dead, exhibiting no signs of brain activity, breathing, heartbeat or muscle movement; but returns to normal function hours after thawing. As a consequence of freezing, wood frogs must also endure extreme cellular dehydration, hyperglycemia and anoxia. The current study investigates the effects of the dehydration/rehydration and anoxia/recovery cycles in regulating autophagy and lysosomal biogenesis in wood frog liver and skeletal muscle. The results show that components of the autophagosome biogenesis machinery are significantly downregulated in liver during anoxia/reoxygenation, and in muscle during anoxia/reoxygenation and dehydration/rehydration. However, select components of the autophagosome machinery are upregulated in response to dehydration in wood frog liver; suggesting the formation of few mature autophagosomes that could engulf damaged macromolecules. Components of the lysosomal machinery appear to be either unaffected in response to stress or decrease in response to recovery in both tissues, suggesting potential impairment in autophagosome and lysosome fusion. Autophagic flux under all conditions in liver and muscle was shown to be regulated at multiple levels, mainly by transcriptional control mediated by Transcription Factor EB, microRNAs, and reversible protein modification. Reduced autophagic flux in liver and muscle under all conditions appears to be a coordinated response to conserve energy and contribute to stress-induced metabolic rate depression.

Keywords: Autophagy, lysosomal biogenesis, microRNA, dehydration/rehydration, anoxia/reoxygenation, wood frogs, hypometabolism, wood frog 


\subsection{Introduction}

Natural freeze-tolerance is an astonishing phenomenon that only a handful of species have mastered. One of such species is the wood frog, Rana sylvatica [1,2]. When temperatures decrease, wood frogs burry themselves into subnivean, and start the freezing process as soon as ice from their hibernacula touches their skin $[3,4]$. Nuclear magnetic resonance imaging showed that ice formed on the wood frog skin will slowly propagate inwards to inoculate internal organs [5]. Concurrently, the liver starts the glycogenolysis process and distributes glucose to different organs to be used as a cryoprotectant [6]. In fact, blood glucose levels rise from $5 \mathrm{mM}$ under normal conditions to $\sim 300 \mathrm{mM}$ during freezing, making the wood frog extremely hyperglycemic [7]. Once fully frozen, wood frogs become clinically dead with no signs of brain activity, heartbeat, breathing or muscle movement; but resume to normal soon after thawing [8]. Freezing is also associated with dehydration, because cells move their intracellular water to the extracellular space to prevent internal ice formation in efforts to maintain cellular integrity [9]. During this time, the wood frogs could lose up to $70 \%$ of their total body water and become extremely dehydrated [9]. Moreover, complete cardiac arrest after the full onset of freezing and the freezing of blood itself render the wood frog fully ischemic, therefore freeze-tolerant frogs must also be anoxia tolerant [10]. Indeed, wood frogs have been shown to tolerate up to $40 \%$ dehydration [11], 48-hour anoxia [12] and glucose loading ( 200 mM plasma glucose levels) [13] independently of freezing; thereby providing a novel and multifaceted model for studying adaptations to freezing.

In this study, the effects of dehydration/rehydration and anoxia/reoxygenation treatments on autophagy was investigated in wood frogs. Tolerance toward dehydration is an evolutionarily conserved phenomenon across many species, especially anurans in 
response to dry and hot temperatures [14]. Dehydration decreases cell volume and increases both osmolality and ionic strength in the cytoplasm as it shrinks the cell. To prevent excessive cellular shrinkage and damage to plasma membrane bilayer, wood frogs use colligative cryoprotectants [9]. Indeed, exposure to dehydrating condition stimulated the production and accumulation of glucose in liver, heart, brain, muscle and kidney, with levels increasing by 9 to $\sim 313$ folds (with levels peaking at 1092, 1409, and $1263 \mathrm{nmol} / \mathrm{mg}$ protein in brain, heart and liver respectively) compared to control values [9]. The same study also reported a concurrent decrease in liver glycogen levels and increase in lactate production; the latter suggesting that wood frogs switch to anaerobic energy metabolism as blood viscosity increases and blood volume decreases. Dehydration also stimulates the production of urea, another colligative cryoprotectant that not only retards water loss, but also contributes to increasing the body fluid osmolality that is needed to combat excessive cell volume reduction $[15,16]$. Indeed, Alaskan wood frogs increase their plasma urea levels from $\sim 10$ to $\sim 86 \mu \mathrm{mol} / \mathrm{mL}$ in late summer to over $187 \mu \mathrm{mol} / \mathrm{mL}$ under dehydrating conditions [17]. Less information is available on metabolism in anoxia exposed wood frogs, but it has been reported that exposure to anoxia does not initiate a strong hyperglycemic response as seen during freezing or dehydration $[18,19]$. Interestingly, a lack of hyperglycemic response was also observed in anoxic turtle livers; thereby suggesting that perhaps hyperglycemia is not a suitable response to anoxia [20]. Understandably, a strong hyperglycemic response is required for cryoprotective purposes during freezing and dehydration; a response that may not be necessary under anoxic conditions since there are no evidence of cell volume reduction, cellular shrinkage, or osmotic pressure. Therefore, glucose levels normally 
present in anoxic wood frogs would be allocated for energy production rather than cryoprotection. In support of this, a recent study on the phosphoproteome of the anoxic wood frog liver proposed that the unchanged levels of phosphofructokinase-2 potentially allows for anaerobic glycolysis to move forward until fuel levels are depleted [21]. Current literature lacks information on urea metabolism in anoxic wood frogs; however, it appears that enzymes that regulate the urea cycle are preferentially more active in frozen and dehydrated rather than anoxic wood frog livers [21]. This finding is also supported by findings in turtle liver, where biosynthesis of urea drops to zero in anoxic animals [20]. Although dehydration and anoxia appear to be different from each other, they appear to share common features and elicit some common responses, one of which is selective reduction in metabolic rates.

Under anoxic and dehydrating conditions, wood frogs have limited access to oxygen; as such, they switch to anaerobic metabolism for energy production [22]. However, anaerobic metabolism cannot sustain full metabolic functions, therefore wood frogs strategically reduce their metabolic rates to allocate the limited energy available only for processes that promote survival, while reducing or halting all other detrimental or non-essential ones. For example, cell destructive processes such as apoptosis [23] and energy expensive proliferative processes such as the cell cycle [24] are attenuated, whereas pro-survival targets such as protein chaperons, antioxidant enzymes, and antioxidant metabolites are up-regulated $[25,26]$. Strict regulation of metabolic rate depression (MRD) occurs at multiple biological levels: at the transcriptional level, epigenetic modifications can alter the accessibility of DNA to the transcriptional machinery; thereby preventing transcription [27]. At the post-transcriptional level, wood 
frogs have been shown to use microRNA-mediated mechanisms to control the availability and levels of specific transcripts, where perfect complementarity between mRNA-microRNA would lead to transcript degradation and partial complementary would lead to transcript storage [28]. At the translational level, strict regulation of the ribosomal machinery suppresses protein synthesis to conserve energy [29], and at the post-translational level, reversible protein modification functions to stabilize/enhance the enzymatic activity of a given protein or render it inactive until it is needed [22]. Entry into and exit out of a hypometabolic states depends on perfect synchrony between all these biological levels; as such, any dysregulation at one or multiple level could lead to catastrophic outcomes in wood frogs.

Macroautophagy (hereafter referred to as autophagy) is an energy expensive, catabolic process where defective or dysfunctional cytoplasmic organelles and proteins are degraded to generate metabolic substrates under different conditions [30]. Although initial studies on autophagy focused on its role during starvation, recent studies have investigated the importance of this process in maintaining cellular homeostasis during a stress response and under different conditions [31-33]. Several studies focus on the regulation of autophagy during ischemia/reperfusion and normoglycemia/hyperglycemia events; however, some of these findings are conflicting. For example, one study showed that excessive autophagy in hippocampal neurons treated with hypoxia/ischemia led to cell death and inhibition of autophagy reversed this process [34] whereas another reported that inhibition of autophagy accelerated ischemia-induced liver injury and induction of autophagy in the same model decreased the levels of reactive oxygen species and necrotic cell death [35]. Despite these conflicting reports, it is reasonable to propose 
that basal autophagic activity is beneficial as it could prevent the accumulation of damaged proteins, organelles, and macromolecules inside the cell.

Autophagy is a multiphase process (initiation, nucleation, elongation and fusion) involving the fusion of a double-membraned vesicle (autophagosome) that engulfs defected cytoplasmic material and then fuses with lysosomes, where the cargo will be degraded [32,36]. A detailed explanation of the sequence of events that occur in autophagy is outline in Figure 1.1. Given the complexity and the importance of autophagy, this process is regulated at multiple biological levels. For example, at the transcriptional level, the induction of autophagy and lysosomal biogenesis are controlled by multiple epigenetic modifications (histone methylation [37], acetylation [38]), and multiple transcription factors (for review [39]), one of which is Transcription factor EB (TFEB). TFEB is a member of the MiT/TFE (microphthalmia/transcription factor E) family and a master regulator of both autophagy and lysosomal biogenesis [40-42]. The binding of TFEB to DNA occurs at a conserved E-box sequence (CACGTG), flanked by specific nucleotides (GTCACGTGAC) that are characteristic to the Coordinated Lysosomal Expression And Regulation (CLEAR) motif [40,41]. Bioinformatic analysis revealed the existence of the CLEAR motif within the promoter region of many genes involved in autophagy initiation and lysosome fusion (BECLIN1, NRBF2, RAB7a, SQSTM1, various VPS proteins, etc.), lysosomal hydrolases and accessory proteins (ASAH1, CTSA, GALNS, HEXA/B, etc.), lysosomal membrane formation (CLCN7, CTNS, MCOLN1, TMEM55B), lysosomal acidification (ATP6 variants), and nonlysosomal proteins that are involved in lysosomal biogenesis (SMURF1, NAGPA, etc.) 
$[40,41]$. As such, activation of TFEB plays a significant role in regulating autophagy and must therefore be strictly regulated to prevent excessive autophagic influx.

Beside regulation at the transcriptional level, autophagy is also regulated at the post-transcriptional level by microRNAs (miRNA) [43]. MiRNAs are highly conserved, small non-coding, single stranded RNAs ( $\sim 22$ nucleotides) that bind to the 3'untranslated region (UTR) of specific mRNA transcripts, and based on the degree of complementarity, they can either degrade the transcript or sequester it in stress granules and p-bodies for future retrieval [44]. Given the importance of miRNAs in regulating mRNA translation, they appear to be a feasible and an energy efficient way of controlling protein synthesis during MRD. Indeed, multiple studies in our laboratory have demonstrated the involvement of microRNAs in regulating mRNA translation in different animals that undergo MRD [28,45-48] and also as a response to whole body freezing in wood frogs [45]. The versatility of miRNAs allows them to bind to multiple target transcripts and regulate their translation under different conditions. In theory, given their conserved nature and degree of complementarity to a given transcript, it is reasonable to assume that the same miRNAs under one condition will likely bind to the same transcript under different conditions. For example, overexpression of miR-17 resulted in a significant decrease in protein levels of p62, whereas inhibition of this miRNA increased the protein levels of p62 in human chondrosarcoma cells [49]. Similarly, overexpression of miR-17 (along with its AAAGUGC-seed containing miR-20, 93, and 106) resulted in a decrease protein levels of p62 in hematopoietic progenitors [50].

Autophagy can also be regulated by post-translational modifications (PTMs) [51]. Reversible protein modification is another energy efficient way of controlling protein 
activity that results in either stabilization of the active form or inhibition of enzymatic activities [52]. There are numerous known (phosphorylation, glycosylation, acetylation, methylation, lipidation, etc.) and unknown modifications that can either modify the proteins once or in sequential and combinatorial manner to regulate their activity [51,52]. Autophagy-regulating proteins (ATGs) are subjected to multiple forms of PTMs, namely phosphorylation, acetylation, ubiquitination, and lipidation), where the identity of the modification will dictate the functional role of the corresponding proteins [53,54].

This study evaluated the effects of $40 \%$ dehydration, rehydration and $24 \mathrm{~h}$ anoxia and $4 \mathrm{~h}$ aerobic recovery on autophagy and lysosomal biogenesis at various biological levels. The overall results demonstrate a strong attenuation in autophagic degradation (marked by reduction in protein levels of the autophagic machinery) during anoxia, reoxygenation, and rehydration (less so during dehydration) in liver and more strongly in skeletal muscle in all conditions. Current results also demonstrate that the level at which autophagy is regulated is highly dependent on the type of tissue and the nature of the stress treatment.

\subsection{Methods}

\section{Animal treatment}

Animal treatment was carried out as described previously [11]. Briefly, adult male wood frogs were collected from breeding ponds near Ottawa, Ontario during the spring, kept on ice and transported to Carleton University. Frogs were washed in a tetracycline bath, housed in plastic boxes lined with damp sphagnum moss, and acclimated at $5{ }^{\circ} \mathrm{C}$ for two weeks. The control group (normoxic and hydrated wood frogs) was sampled from this condition. Animals were euthanized by pithing and liver and hind leg skeletal muscle 
samples were excised quickly, and flash frozen in liquid nitrogen before being stored at $80{ }^{\circ} \mathrm{C}$. All animal care, experimental and euthanasia protocols were carried out as previously approved by the Carleton University Animal Care Committee (Protocol no. 106935) following /guidelines set by the Canadian Council on Animal Care.

\section{Anoxia/reoxygenation exposure}

Sealed plastic containers with a layer of damp paper towel on the bottom (wetted with water previously bubbled with $100 \%$ nitrogen gas for $30 \mathrm{~min}$ ) were held on crushed ice. The lid of each containers had two ports: one that would allow $100 \%$ nitrogen gas in and one to vent excess air out. Nitrogen gas was flushed into the containers for approximately $30 \mathrm{~min}$. and then a group of 4-5 frogs was placed in each jar and lids were sealed. The jars were flushed with nitrogen gas for another $15 \mathrm{~min}$, before being transferred to $5^{\circ} \mathrm{C}$ incubators for $24 \mathrm{~h}$. After the $24 \mathrm{~h}$ exposure period (hereafter anoxia), half of the containers were removed sequentially, placed on ice again, and flushing with nitrogen gas was reinstated while frogs were euthanized and sampled, as above. For the reoxygenated group, $24 \mathrm{~h}$ anoxia-exposed frogs in other containers were transferred quickly to new containers lined with damp paper towel (wetted with normoxic water) and allowed to recover in normal air for $4 \mathrm{~h}$ in $5{ }^{\circ} \mathrm{C}$ incubators. Following this, containers were placed on ice and animals were sampled as above.

\section{Dehydration/rehydration exposure}

Other groups of acclimated frogs were individually weighed, ranked based on their weight, and placed in dry plastic containers (10 frogs per box) without lids and then replaced in $5{ }^{\circ} \mathrm{C}$ incubators. Over time, frogs lost water through evaporation, and their weights were monitored at intervals. The amount of body water lost was calculated using 
the following equation $\left(\mathrm{M}_{\mathrm{i}}-\mathrm{M}_{\mathrm{d}}\right) /\left(\mathrm{M}_{\mathrm{i}} \mathrm{x} \% \mathrm{H}_{2} \mathrm{O}\right)$ where $\mathrm{M}_{\mathrm{i}}$ is the frog initial mass, $\mathrm{M}_{\mathrm{d}}$ is the mass at each weighing, and $\% \mathrm{H}_{2} \mathrm{O}$ is the percentage of total body mass of control, normally hydrated frogs that is water (for control wood frogs this is $80.8 \pm 1.2 \%$ ) [55]. Under these conditions, wood frogs lost $\sim 0.5 \%$ of their total body water per hour. Half of the dehydrated frogs were sampled when they reached $40 \%$ of total body water lost. The remaining $40 \%$ dehydrated frogs were transferred to new boxes with $\sim 0.5 \mathrm{~cm}$ of water in the bottom to rehydrate overnight $(\sim 16 \mathrm{~h})$ before being sampled.

\section{Total protein extraction for Western blot analysis}

Total protein extraction procedures were followed as previously published [56]. Approximately $50 \mathrm{mg}$ of frozen liver or muscle samples $(\mathrm{n}=4$ independent biological replicates obtained from different animals) were homogenized 1:2 w/v in ice cold homogenization buffer containing 20 mM HEPES pH 7.4, $100 \mathrm{mM} \mathrm{NaCl}, 10 \mathrm{mM} \mathrm{NaF}, 1$ $\mathrm{mM} \mathrm{Na}_{3} \mathrm{VO}_{4}, 10 \mathrm{mM} \beta$-glycerophosphate, $0.1 \mathrm{mM}$ EDTA, and $1 \mu \mathrm{L}$ of protease inhibitor cocktail (Bioshop, cat. No. PICoo1.1) per mL of homogenization buffer with a few crystals of PMSF (phenylmethylsulfonyl fluoride) added. Samples were homogenized using a Polytron homogenizer for 15-20 seconds. Samples were then centrifuged at $12000 \mathrm{xg}$ for $15 \mathrm{~min}$ at $4{ }^{\circ} \mathrm{C}$, supernatants were collected, and total soluble protein concentrations were determined using the Bio-Rad protein assay (Bio-Rad, cat. No 5000006). Total protein concentration of control, stress and recovery samples were then standardized to a common concentration using homogenization buffer. Aliquots of total protein extracts were then mixed 1:1 v:v with 2X SDS loading buffer (100 mM Tris-HCl, $10 \%$ v:v glycerol, $10 \% \quad \mathrm{v}: \mathrm{v} \quad 2-\beta$-mercaptoethanol, $4 \% \quad \mathrm{w}: \mathrm{v}$ SDS and $0.2 \% \quad \mathrm{w}: \mathrm{v}$ 
bromophenol blue), and then samples were boiled in a water bath for $5 \mathrm{~min}$. After cooling on ice for $5 \mathrm{~min}$, samples were stored at $-80^{\circ} \mathrm{C}$ until use.

\section{Western blotting}

Equal protein amounts from control, stress and recovery samples for each tissue were loaded onto SDS-PAGE gels, $8-10 \%$ depending on protein molecular weight (M.W.), and electrophoresis was carried out using a Bio-Rad Mini Protean III system (60-150 min depending on protein M.W.) as previously explained [11]. Once the desired separation was reached, proteins were transferred onto PVDF (polyvinylidene difluoride) membranes at $160 \mathrm{~mA}$ for $45-120 \mathrm{~min}$, depending on the protein's molecular weight. Following transfer, membranes were washed with TBST (20 mM Tris base pH 7.6, 150 $\mathrm{mM} \mathrm{NaCl}, 0.05 \% \mathrm{v} / \mathrm{v}$ Tween-20) for $5 \mathrm{~min}$ while rocking at room temperature (RT). Membranes were blocked with non-fat dry milk dissolved in TBST $(1-10 \%$ as appropriate) to prevent non-specific binding followed by washing for $3 \times 5$ min in TBST while rocking at RT. Membranes were then incubated with $5 \mathrm{~mL}$ of primary antibody at $4^{\circ} \mathrm{C}$ while rocking overnight, followed by washing as above and then incubation with 5 $\mathrm{mL}$ of secondary antibody conjugated to hose-radish peroxidase enzyme for $30 \mathrm{~min}$ at $\mathrm{RT}$ while rocking. Membranes were washed again as above and visualized via enhanced chemiluminescence reagents (ECL). Subsequently, membranes were stained with Coomassie blue and reimaged to obtain a relative quantification of the total protein in each lane transferred to the membrane. The estimated total protein in each lane will be used to normalize the intensity of chemiluminescent bands of the protein of interest to adjust for slight differences in protein loadings in each gel. 
The following antibodies were purchased from GeneTex: anti-ULK1 (GTX132669), anti-p-ULK1 (S758) (GTX132654), anti-ATG14 (GTX128166), antiLC3B (GTX127375). Antibodies purchased from Abclonal were: anti-p-ULK1(S555) (AP0760), anti-ATG4a (AP1808a), anti-TFEB (A7311), anti-ATP6V0D1 (A4271). The following antibodies were from Abgent: anti-Beclin1 Abgent (AP1818a), anti-ATG3 (AP1807a), anti-ATG5L (AP1812a). The anti-p-Beclin1 (S93) antibody was purchased from Cell Signaling (14717) and anti-p62 was from Biolegend (647701).

Total protein extraction for DNA-Protein interaction enzyme-linked immunosorbent assay (DPI-ELISA)

Samples of frozen tissue $(\sim 50 \mathrm{mg}$ each, $\mathrm{n}=4$ independent biological replicates from different animals) from control, stress and recovery groups were homogenized in 1:5 w/v cold lysis buffer (EMD Millipore, cat. No. 43-045) containing $10 \mathrm{mM} \beta$ glycerophosphate, $10 \mathrm{mM} \mathrm{NaF}, 1 \mathrm{mM} \mathrm{Na} \mathrm{VO}_{4}$, and $1 \%$ protease inhibitor cocktail per $\mathrm{mL}$ of buffer for 15-20 sec using a Polytron homogenizer. Samples were incubated on ice for $\sim 40$ min with intermittent vortexing followed by centrifugation at $14,000 \mathrm{xg}$ at $4{ }^{\circ} \mathrm{C}$ for $20 \mathrm{~min}$. Then supernatants were collected and stored at $-80^{\circ} \mathrm{C}$ until use.

\section{DPI-ELISA}

The DPI-ELISA procedure was followed as previously published [56]. DNA oligonucleotides containing the TFEB consensus sequence were purchased from Integrated DNA Technologies (Integrated DNA Technologies, Coralville, IA, USA). The

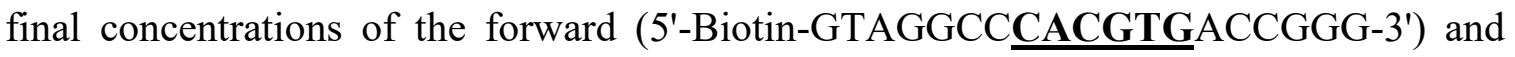

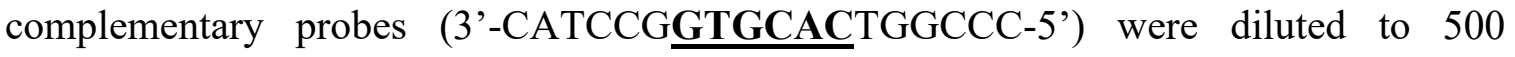


$\mathrm{pmol} / \mu \mathrm{L}$ using double distilled water. The single stranded probes were combined in a 1:1 ratio, before being placed in a thermocycler set to $95^{\circ} \mathrm{C}$ for 10 minutes to hybridize. The double stranded probes were then cooled RT, and diluted with 1x PBS (phosphate buffer saline, $137 \mathrm{mM} \mathrm{NaCl}, 10 \mathrm{mM} \mathrm{Na} 2 \mathrm{HPO}_{4}, 2.7 \mathrm{mM} \mathrm{KCl}$, and $2 \mathrm{mM} \mathrm{KH}_{2} \mathrm{PO}_{4}, \mathrm{pH}$ 7.4). A volume of $50 \mu \mathrm{L}$ of the diluted probe was added to each microplate well (40 pmol probe/well) coated with streptavidin (R\&D Systems, Minneapolis, MB, Canada), and wells were incubated for 1 hour at RT without agitation. Following incubation, excess unbound probes were removed, and each well was washed $2 \mathrm{X}$ with $200 \mu \mathrm{L}$ of wash buffer (1X PBS containing 0.1\% Tween-20) and one time with 1X PBS. Probed microplates were kept at $4^{\circ} \mathrm{C}$ until use.

The concentration of total proteins extracted for DPI-ELISA was measured using a Bio-Rad assay immediately before the experiment was performed. Briefly, equal concentrations $(n=4$ independent biological replicates obtained from different animals, run in duplicates) of the liver and muscle were combined with transcription factor binding buffer containing $50 \mathrm{mM} \mathrm{KCl}, 20 \mathrm{mM}$ DTT, $10 \mathrm{mM}$ HEPES, $3 \mathrm{mM} \mathrm{MgCl} 2,0.5$ mM EDTA, $0.5 \mathrm{mg} / \mathrm{mL}$ BSA, 10\% v:v glycerol, $0.05 \%$ NP-40, pH 7.9 and $1 \mu \mathrm{L}$ of salmon sperm DNA $(1 \mu \mathrm{g} / \mathrm{mL})$ per well and mixed briefly. The streptavidin coated microplate wells were purchased from R\&D systems (R\&D Systems, cat no. CA73521134). A volume of $50 \mu \mathrm{L}$ (containing $15-25 \mu \mathrm{g}$ of total protein extract) was added to each probed well, and the microplate was incubated for $1 \mathrm{~h}$ at RT with mild shaking. Negative control wells contained the same assay components, but an equal volume of lysis buffer (EMD Millipore, cat. no. 43-045) was added in lieu of proteins samples. Following incubation, excess sample was discarded, and the wells were washed $3 \mathrm{X}$ with wash 
buffer. To detect the amount of TFEB bound, $60 \mu \mathrm{L}$ of 1:1000 anti-TFEB antibody diluted in 1X PBST (1X PBS containing 0.05\% Tween-20) was added to each well and the microplate was incubated for $1 \mathrm{~h}$ at RT without agitation. Following the removal of excess primary antibody and washing, $60 \mu \mathrm{L}$ of 1:2000 anti-rabbit $\operatorname{IgG}$ conjugated to HRP was added to each well, and the plate was incubated for another hour at RT without agitation. Excess secondary antibody was removed, the wells were washed and $60 \mu \mathrm{L}$ of TMB (tetramethylbenzidine; Bioshop, cat. no. TMB333.100) was added to each well. Once the desired intensity was reached, $60 \mu \mathrm{L}$ of stop solution $(1 \mathrm{M} \mathrm{HCl})$ was used to terminate the reaction. The absorbance of each well was measured at $450 \mathrm{~nm}$ (with reference wavelength of $655 \mathrm{~nm}$ ) using a Multiskan spectrophotometer (Thermo Electron Corporation, Waltham, MA, USA).

To ensure TFEB probe specificity, the same assay was performed using wells that either lacked DNA probe, protein sample, or TFEB antibody. The optimum protein concentration to be used for each tissue was determined by performing the same assay using pooled samples at varying concentrations. The concentrations that had the lowest protein amount and the highest signal to noise ratio were used for each tissue in the quantification run.

\section{Total RNA extraction}

Total RNA was extracted as previously published [48]. Briefly, $50 \mathrm{mg}$ of frozen liver and muscle ( $\mathrm{n}=4$ independent biological replicates from different animals) was homogenized in $1 \mathrm{~mL}$ of Trizol reagent (Invitrogen, cat. no. 15596-018) using a Polytron homogenizer and subsequently samples were incubated for $5 \mathrm{~min}$ on ice. Then $200 \mu \mathrm{L}$ of chloroform was added to each tube and mixed for $15 \mathrm{sec}$. Samples were then centrifuged 
at $10,000 \mathrm{rpm}$ at $4{ }^{\circ} \mathrm{C}$ for $15 \mathrm{~min}$. The upper aqueous layer was removed and combined with another $200 \mu \mathrm{L}$ of chloroform. Samples were mixed and centrifuged as above, and the resulting aqueous layer containing total RNA was collected. Each sample was mixed with $500 \mu \mathrm{L}$ of isopropanol and placed on ice for $\sim 15 \mathrm{~min}$ to precipitate RNA. Samples were then centrifuged at $12,000 \mathrm{rpm}$ for $15 \mathrm{~min}$ at $4{ }^{\circ} \mathrm{C}$, the supernatant was discarded, and resulting pellets were washed with $1 \mathrm{~mL}$ of cold ethanol (70\%). Samples were then centrifuged at 7,500 rpm for $5 \mathrm{~min}$ at $4{ }^{\circ} \mathrm{C}$, the supernatant was discarded, and remaining pellets were air dried for 15 min at RT. Pellets were resuspended in autoclaved double distilled water (30-50 $\mu \mathrm{L}$ depending on pellet size), and the quality of the extracted RNA was determined by the $260 / 280 \mathrm{~nm}$ ratio using a NanoDrop spectrometer (Fisher Scientific, Wilmington, DE, USA); samples with ratios higher than 1.8 were used for further analysis. The integrity of the extracted RNA was also tested by running equal concentrations of each sample on a $1 \%$ agarose gel stained with SYBR Green to visualize the $28 \mathrm{~S}$ and the $18 \mathrm{~S}$ ribosomal bands. RNA was stored at $-80^{\circ} \mathrm{C}$ for future use.

\section{MicroRNA polyadenylation and stem-loop reverse transcription}

MicroRNA polyadenylation, stem-loop reverse transcription and quantification were performed as previously published [48]. Frozen RNA samples were thawed on ice, and their concentration was again measured using a NanoDrop spectrophotometer. All RNA concentrations were standardized to $0.8 \mu \mathrm{g} / \mu \mathrm{L}$ and used for subsequent steps. The PolyA tailing kit (Epi-Bio, Cat no. PAP5104H) was used for polyadenylation. Briefly, a total of $10 \mu \mathrm{L}$ of reaction mixture [1 $\mu \mathrm{g}$ of total RNA, $2 \mathrm{U}$ of E. coli poly (A) polymerase and $1 \mathrm{mM}$ ATP] diluted in reaction buffer $\left(10 \mathrm{mM} \mathrm{MgCl}_{2}, 0.25 \mathrm{M} \mathrm{NaCl}, 0.1 \mathrm{M}\right.$ Tris- $\mathrm{HCl}$ $\mathrm{pH}$ 8.0) was incubated for $30 \mathrm{~min}$ at $37{ }^{\circ} \mathrm{C}$ to enable microRNA polyadenylation. The 
reaction was then heated to $95{ }^{\circ} \mathrm{C}$ for 5 mins to terminate the process and then incubated on ice for $\sim 15 \mathrm{~min}$. To reverse transcribe the polyadenylated microRNA, $10 \mu \mathrm{L}$ of each sample was combined with $5 \mu \mathrm{L}$ of $250 \mathrm{pM}$ stem-loop adapter primer (Table 2.1) and incubated at $95{ }^{\circ} \mathrm{C}$ for $5 \mathrm{~min}$, followed by $60^{\circ} \mathrm{C}$ for $5 \mathrm{~min}$. Samples were then centrifuged and immediately placed on ice for $\sim 5 \mathrm{~min}$. For the reverse transcription step, $15 \mu \mathrm{L}$ of polyadenylated microRNA was combined with $10 \mu \mathrm{L}$ of master mix containing $1 \mu \mathrm{L}$ (2 units) of mouse Maloney leukemia virus reverse transcriptase (Invitrogen, cat. no. 28025013), $1 \mu \mathrm{L}$ of $25 \mathrm{mM}$ deoxynucleotide triphosphate (BioShop, Burlington, ON, CA), 2 $\mu \mathrm{L}$ of $0.1 \mathrm{M}$ of DTT, $1 \mu \mathrm{L}$ of autoclaved double distilled water, and $5 \mu \mathrm{L}$ of $5 \mathrm{x}$ First Strand buffer (ThermoFisher Scientific, cat. no. 18080044). Samples were then placed in a thermocycler and subjected to the following protocol: $16^{\circ} \mathrm{C}$ for $30 \mathrm{~min}, 42{ }^{\circ} \mathrm{C}$ for 30 $\min$, and $85{ }^{\circ} \mathrm{C}$ for $5 \mathrm{~min}$. Samples were then held at $10^{\circ} \mathrm{C}$ for $\sim 15 \mathrm{~min}$, serially diluted in autoclaved double distilled water and stored at $-20{ }^{\circ} \mathrm{C}$ until needed.

\section{Relative microRNA quantification}

A select group of microRNAs known to be involved in autophagy regulation was curated from literature. Their sequences were retrieved from miRbase (http://www.mirbase.org/), compared to multiple vertebrate species and only the $100 \%$ conserved ones were used for quantification. MicroRNA-specific primers, the universal primer and reference primers were purchased from Integrated DNA Technologies (Coralville, IA, USA) (Table 2.1). Relative microRNA amplification was carried out using the MIQE guidelines [57] using a CFX Connect ${ }^{\mathrm{TM}}$ Real-Time PCR Detection System, as described previously [45]. Briefly, $2 \mu \mathrm{L}$ of cDNA was combined with $18 \mu \mathrm{L}$ of master mix containing: $10.12 \mu \mathrm{L}$ autoclaved double distilled water, $4 \mu \mathrm{L}$ of $1 \mathrm{M}$ 
trehalose (BioShop, cat. No. TRE222), $2 \mu \mathrm{L}$ of qRT-PCR buffer (100 mM Tris-HCl pH 8.5, $500 \mathrm{mM} \mathrm{KCl}, 20 \mathrm{mM} \mathrm{MgCl} 2,2 \mathrm{mM}$ dNTPs, $100 \mathrm{nM}$ Fluorescein and 1.5\% Triton $\mathrm{X}-100$ ), $0.5 \mu \mathrm{L}$ of $100 \%$ formamide (BioShop, cat no. FOR001), $0.5 \mu \mathrm{L}$ of microRNAspecific forward primer $(25 \mathrm{mM}), 0.5 \mu \mathrm{L}$ of universal primer $(25 \mathrm{mM}), 0.1 \mu \mathrm{L}$ of SYBR green diluted in dimethyl sulfoxide (Invitrogen, cat. no. S7585), $0.16 \mu \mathrm{L}$ of dNTPs (25 $\mathrm{mM})$ and $0.13 \mu \mathrm{L}$ of Taq polymerase $(5 \mathrm{U} / \mu \mathrm{L}$, BioShop, cat. no. TAQ001.1). The following program was used for microRNA amplification: initial denaturation for $3 \mathrm{~min}$ at $95{ }^{\circ} \mathrm{C}, 40$ cycles of $15 \mathrm{sec}$ at $95^{\circ} \mathrm{C}$ followed by $1 \mathrm{~min}$ at $60^{\circ} \mathrm{C}$. All RT-qPCR analyses were run in duplicates, subjected to post-run melt curve analysis, and only reactions amplifying a single peak were used for quantification.

\section{microRNA binding-site prediction}

The potential binding sites of miR-17-5p to the $p 62$ mRNA were predicted using SFold 2.2 STarMir software (http://sfold.wadsworth.org/cgi-bin/starmirtest2.pl) [58]. Since the wood frog is not genome sequenced, the mRNA sequences of another frog (Xenopus laevis) were used as input sequences for the prediction. The HITS-CLIP based model (mouse) was used to determine the predicted binding sites. mRNA cDNA synthesis

First strand cDNA synthesis was carried out by combining $1 \mu \mathrm{g}$ of RNA and $1 \mu \mathrm{L}$ of $200 \mathrm{ng} / \mu \mathrm{L}$ Oligo(dT) (Sigma Genosys) diluted to a final volume of $10 \mu \mathrm{L}$ with autoclaved $\mathrm{ddH}_{2} \mathrm{O}$ and incubated at $65{ }^{\circ} \mathrm{C}$ for $5 \mathrm{~min}$ in a thermocycler (iCycler,Biorad). Samples were then chilled on ice for 2 min, combined with $4 \mu \mathrm{L}$ of $5 \mathrm{x}$ First Strand Buffer (Invitrogen), $2 \mu \mathrm{L}$ of $100 \mathrm{mM}$ DTT, $1 \mu \mathrm{L}$ of MMLV reverse transcriptase (Invitrogen), 
and $1 \mu \mathrm{L}$ of $25 \mathrm{mM}$ dNTPs (Bioshop), and incubated in a thermocycler at $42{ }^{\circ} \mathrm{C}$ for 60 min. Samples were then chilled on ice, serially diluted, and stored at $-20{ }^{\circ} \mathrm{C}$ until needed. Primer design \& mRNA quantification

Given that the wood frog genome is not sequenced, the forward and reverse primers for p62 (sequestosome 1; sqstm1) were designed using the Xenopus tropicalis p62 gene sequence as input in PrimerQuest (https://www.idtdna.com/pages/tools/primerquest). Only the forward and reverse primers that were conserved across multiple vertebrate species were used for quantification using a CFX Connect ${ }^{\mathrm{TM}}$ Real-Time PCR Detection System (Bio-Rad, cat. No. 1855201) following MIQE guidelines [57]. Each reaction $(20 \mu \mathrm{L}$ in total) contained $10.12 \mu \mathrm{L}$ of $\mathrm{ddH}_{2} \mathrm{O}, 4 \mu \mathrm{L}$ of $1 \mathrm{M}$ trehalose (BioShop, Cat no. TRE222), $2 \mu \mathrm{L}$ of 10X qRT-PCR buffer (500 mM KCl, 100 mM Tris- $\mathrm{HCl} \mathrm{pH}$ 8.5, 20 mM MgCl $2,2 \mathrm{mM}$ dNTPs, 1.5\% Triton X100, and $100 \mathrm{nM}$ fluorescein), $0.5 \mu \mathrm{L}$ of $100 \%$ formamide (Bioshop, Cat no. For001), 0.5 $\mu \mathrm{L}$ each of forward and reverse primers (both $0.3 \mathrm{nmol} / \mu \mathrm{L}$ ), $0.16 \mu \mathrm{L}$ of $25 \mathrm{mM}$ dNTPs, $0.125 \mu \mathrm{L}$ Taq Polymerase (5U/ $\mu \mathrm{L}$, BioShop, Cat no. Taq001.1), $0.1 \mu \mathrm{L}$ of $100 \mathrm{X}$ SYBR Green diluted with DMSO (Invitrogen, Cat no.S7585), and $2 \mu \mathrm{L}$ of cDNA. The following amplification program was used for p62 transcript amplification (Table 2): touchdown PCR for 3 min at $95{ }^{\circ} \mathrm{C}, 9$ cycles of $10 \mathrm{sec}$ at $95{ }^{\circ} \mathrm{C}, 20 \mathrm{sec}$ at $56^{\circ} \mathrm{C}, 20 \mathrm{sec}$ at $72{ }^{\circ} \mathrm{C}$. The amplification process then was carried out as follows: 39 cycles of $10 \mathrm{sec}$ at $95^{\circ} \mathrm{C}, 20 \mathrm{sec}$ at $54{ }^{\circ} \mathrm{C}$ and $20 \mathrm{sec}$ at $72{ }^{\circ} \mathrm{C}$. The sequence and amplification protocols for $\beta$-actin (Table 2) were performed as described previously [59]. The efficiency of each set of primers was tested prior to the quantification run, and the specificity of the amplification process 
was tested by performing a melt-curve analysis after each run. All primers used in this study produced a single product, and each sample was run in duplicate.

\section{Quantification and statistics}

For western blot analysis, the band densities from ECL signals as well as the corresponding Coomassie blue-stained membranes were determined using the GeneTools software (Syngene, Frederick, MD, USA). The ECL signal for each band of interest was standardized against a group of stably expressed bands (well separated from the band of interest) in the same lane on the Coomassie stained membrane; this accounted for minor variations in protein loading. This method of standardization was reported to more reliable than using a single housekeeping protein as reference [60]. The fold changes in protein levels observed during $24 \mathrm{~h}$ anoxia, $4 \mathrm{~h}$ aerobic recovery, $40 \%$ dehydration or rehydration were calculated relative to the control group, which was set to 1 . Histograms show mean relative protein expression levels (mean \pm SEM) obtained from $n=4$ independent biological replicates. Statistical significance as compared with controls $(p<0.05)$ was evaluated assessed by a one-way ANOVA with a Dunnett's post-hoc test, using the RBIOPLOT statistical and graphical R package [61].

The absorbance values obtained from the DPI-ELISA experiments were measured at $450 \mathrm{~nm}$, using $655 \mathrm{~nm}$ as the reference wavelength in a Multiskan spectrophotometer (Thermo Electron Corporation, Waltham, MA, USA). The relative DNA binding levels of TFEB observed during 24-h anoxia, 4h aerobic recovery, 40\% dehydration and rehydration are calculated relative to the control group, which was set to 1 . Relative DNA binding levels (mean \pm SEM) were obtained from $n=4$ independent biological replicates. Statistical significance from the control $(\mathrm{p}<0.05)$ were calculated by a One-Way ANOVA 
with Dunnette's post-hoc test, using the RBIOPLOT statistical and graphical R package [61].

For all RT-qPCR analyses, the relative microRNA or mRNA levels were calculated using the comparative $\Delta \Delta \mathrm{Cq}$ method. Briefly, raw $\mathrm{Cq}$ values were linearized $\left(2^{-\mathrm{Cq}}\right)$, and the obtained values were standardized against reference genes that showed stable expression under the experimental conditions tested. Relative microRNA transcript levels in liver were standardized against Snord-24-17bp over the anoxia/recovery and dehydration/rehydration cycles. Relative microRNA transcript levels in muscle were standardized against Snord-74a-15bp over for anoxia/recovery data, and against 5S RNA over the dehydration/rehydration cycle in wood frogs. The relative transcript abundance of the p62 gene was normalized against $\beta$-actin in liver because it showed a stable expression during the anoxia/recovery and dehydration/rehydration cycle in wood frogs. Transcript levels of both mRNA and microRNA over the stress/recovery cycle were expressed relative to the control, which was set to 1 . Relative transcript abundance (mean \pm SEM) was obtained from $\mathrm{n}=3-4$ independent biological replicates. Statistically significant differences from controls were calculated by a one-way ANOVA with Dunnett's post-hoc test, with $\mathrm{p}<0.05$ accepted as significant using the RBIOPLOT statistical and graphical R package [61].

\subsection{Results}

Relative protein expression levels of autophagy markers

Using immunoblotting, relative levels of select proteins involved in various stages of autophagy were measured over the anoxia/recovery and dehydration/rehydration cycles in wood frog liver and skeletal muscle. In liver of anoxia-exposed frogs, levels of 
ULK1 increased significantly by $1.6 \pm 0.10$-fold after $24 \mathrm{~h}$ of anoxia exposure and remained high at $1.67 \pm 0.15$-fold over controls after $4 \mathrm{~h}$ of aerobic recovery (Figure 2.2). Phosphorylation of ULK1 also increased strongly under anoxia with p-ULK1 (S555) and p-ULK (S758) contents rising by $5.1 \pm 0.58$-fold and $2.0 \pm 0.10$-fold, respectively, after $24 \mathrm{~h}$ anoxia exposure and remaining high at $5.5 \pm 0.24$-fold and $2.2 \pm 0.2$-fold over controls, respectively, after $4 \mathrm{~h}$ of aerobic recovery. By contrast, total protein levels of ATG3 and ATG4a decreased significantly by $30 \pm 4 \%$ and $43 \pm 4 \%$, respectively, after anoxia exposure and remained low at $30 \pm 5 \%$ and $32 \pm 10 \%$ compared to controls, after aerobic recovery. Beclin1 levels also decreased significantly by $44 \pm 1.0 \%$ after anoxic exposure, while showing no significant difference from controls after $4 \mathrm{~h}$ aerobic recovery. Levels of ATG5L, p-beclin1 (S93) and ATG14 remained unchanged over the anoxia/recovery cycle, whereas p62 increased by $1.26 \pm 0.07$-fold after anoxia exposure compared to the control group but returned to baseline after aerobic recovery. Levels of LC3BI increased by $5.5 \pm 0.42$-fold after anoxia exposure and remained significantly high at $2.7 \pm 0.24$-fold over controls after aerobic recovery. The same antibody capable of detecting both forms of LC3B failed to detect levels of LC3BII in liver from anoxic/reoxygenated wood frogs.

In liver of wood frogs exposed to $40 \%$ dehydration and rehydration treatments, a similar response was seen for the ULK1 proteins (Figure 2.3). Levels of total ULK1, pULK1 (S555) and p-ULK1 (S758) increased significantly by $2.9 \pm 0.36$-fold, $2.2 \pm 0.11$ fold, and 3.8 \pm 0.42-fold after dehydration, respectively. Total ULK1 and p-ULK1 (S555) levels returned to control values after rehydration whereas p-ULK1 (S758) levels remained high at $3.6 \pm 0.5$-fold over controls. Total protein levels of ATG3, ATG4a and 
beclin1 showed no significant change over the dehydration/rehydration cycle, but levels of ATG5a decreased by $53 \pm 4.7 \%$ during dehydration before returning to baseline after rehydration. Levels of phosphorylated beclin1 (S93) increased by $3.6 \pm 0.35$-fold during dehydration. ATG14 content was unchanged after dehydration but decreased by $48 \pm 9 \%$ after rehydration. Levels of p62, LC3BI and lipidated LC3BI (LC3BII) increased by 2.1 \pm 0.13 -fold, $3.7 \pm 0.63$-fold and $8.1 \pm 1.3$-fold respectively during dehydration compared to controls but returned to baseline after rehydration. The ratio of lipidated (LC3BII) to non-lipidated LC3BI increased by $2.2 \pm 0.9$-fold during dehydration before returning to control levels upon recovery.

To investigate whether the expression of autophagy markers occurs in a tissuespecific manner, we measured the same targets in skeletal muscle over the anoxia/reoxygenation and dehydration/rehydration cycles. Notably, most targets showed a significant downregulation, whereas targets such as p-ULK1 (S758) and LC3BII were below the detection limit under both conditions in the muscle.

In muscle, total levels of ULK1 decreased to $14 \pm 6.4 \%$ after $24 \mathrm{~h}$ anoxia and remained low at to $13 \pm 3.2 \%$ after reoxygenation, as compared with controls (Figure 2.4). By contrast, p-ULK1 (S555) levels were unchanged. ATG3 levels decreased by 43 $\pm 15 \%$ after anoxia treatment before returning to baseline after reoxygenation. Levels of ATG4a, ATG5L and beclin1 decreased by $43 \pm 11 \%, 77 \pm 5.0 \%, 64 \pm 7.5 \%$, respectively, after anoxia exposure treatment, and remained reduced after reoxygenation at $52 \pm 6.4 \%, 73 \pm 5.7 \%$, and $47 \pm 7.8 \%$ as compared to controls. While levels of pbeclin1 (S93) showed no change in anoxia, levels decreased by $84 \pm 1.6 \%$ after reoxygenation. Both ATG14 and p62 contents also decreased significantly by $64 \pm 9.2$, 
and $79 \pm 7.2 \%$, respectively, after anoxic treatment and remained low at $54 \pm 7.6$ and 78 $\pm 3.3 \%$ of controls after reoxygenation. Whereas levels of LC3BI were unchanged after anoxia exposure, levels increased by $4.4 \pm 0.98$-fold compared to the control group after reoxygenation.

The effects of dehydration and rehydration on muscle are shown in Figure 2.5. Total ULK1 was decreased by $70 \pm 8.2 \%$ after dehydration and by $86 \pm 1.6 \%$ after rehydration, whereas levels of p-ULK1 (S555) and ATG3 were unchanged. Levels of ATG4a decreased by $44 \pm 15 \%$ after dehydration but showed no significant change after rehydration compared to controls. ATG5L levels decreased by $44 \pm 7.2 \%$ and remained low $42 \pm 3.9 \%$ after dehydration and rehydration treatments, respectively. Whereas total levels of beclin 1 were unchanged, levels of p-beclin1 (S93) decreased by $58 \pm 3.7 \%$ after dehydration and by $69 \pm 5.7 \%$ after rehydration, as compared with controls, respectively. Levels of ATG14 and p62 remained the same over the dehydration/rehydration cycle, whereas LC3BI showed a significant increase by $2.5 \pm 0.15$-fold during rehydration, compared to the control group.

Relative expression levels of TFEB, ATPVOD1, and DNA binding of TFEB

TFEB is a master regulator of lysosomal biogenesis and regulates the expression of several autophagy-related proteins $[40,41]$. To probe TFEB involvement in dealing with anoxia or dehydration stresses, we examined the DNA binding activity of TFEB using an ELISA method, total TFEB protein levels, and the levels of a key lysosomal downstream target, ATPV0D1. Effects of anoxia or dehydration stresses on TFEB binding to its DNA consensus sequence were assessed in liver and muscle. Anoxia exposure led to a significant $1.5 \pm 0.15$-fold increase in liver TFEB binding to DNA 
compared to controls, but this was reversed after reoxygenation (Figure 2.6A). Dehydration stress had the same effect with DNA binding increasing by $1.5 \pm 0.20$-fold after $40 \%$ dehydration but again returning to baseline after rehydration (Figure 2.6B). A very different response was seen for TFEB in skeletal muscle. DNA binding levels by TFEB decreased by $48 \pm 9.6 \%$ after anoxia exposure and by $69 \pm 4.2 \%$ after reoxygenation, as compared with values for control muscle (Figure 2.6A). Dehydration stress had a similar effect in muscle with a substantial downward trend in TFEB binding in muscle of $40 \%$ dehydrated frogs, and a significant $64 \pm 9.4 \%$ decrease in binding in muscle of rehydrated frogs compared to control (Figure 2.6B).

Total protein expression levels of TFEB as determined by immunoblotting were not affected by anoxia/reoxygenation treatment in liver (Figure 2.7A), whereas levels of ATPV0D1 protein (a transcriptional target of TFEB) were unaffected by anoxia but showed a significant decrease (by $45 \pm 5.4 \%$ ) after $4 \mathrm{~h}$ of aerobic recovery compared to the control group. By contrast with the response to anoxia, dehydration treatment led to a $2.1 \pm 0.26$-fold increase in TFEB protein levels in liver, with a return to baseline levels after rehydration (Figure 2.7B). Levels of ATPV0D1 were unaffected by dehydration but were decreased by $85 \pm 2.8 \%$ after rehydration.

Skeletal muscle showed a different pattern of response by TFEB and ATPV0D1. Anoxia exposure led to a strong decreased in both proteins (Figure 2.8A); TFEB and ATPV0D1 decreased by $56 \pm 7.9 \%$ and by $55 \pm 10.7 \%$ after $24 \mathrm{~h}$ anoxia and fell further by $78 \% \pm 2 \%$ and $61 \pm 8.8 \%$, respectively, after reoxygenation, as compared to controls. Dehydration/rehydration had a lesser effect on both TFEB and ATPV0D1 in skeletal muscle. Dehydration to $40 \%$ of total body water loss did not significantly affect the levels 
of these proteins but both TFEB and ATPV0D1 were significantly decreased by $32 \pm$ $2.4 \%$ and $39 \pm 4.7 \%$, respectively, in muscle after rehydration, compared to controls (Figure 2.8B).

Relative transcript levels of autophagy-regulating microRNAs

A list of ten miRNAs known to regulate components of the autophagic machinery was curated from the literature and measured in wood frog liver and muscle over the anoxia/reoxygenation and dehydration/rehydration cycles. Figure 2.9 shows the relative responses of these miRNAs to stress and recovery in liver using a heat map format. Liver transcript levels of miR-25-3p increased by $1.4 \pm 0.1$-fold after reoxygenation, whereas miR-26-5p levels rose by $1.7 \pm 0.16$-fold and by $1.7 \pm 0.1$-fold after anoxia and reoxygenation, respectively (Figure 2.9A). Levels of miR-30a-5p and miR-30d-5p showed significant increases of $1.6 \pm 0.09$ and $1.5 \pm 0.15$-fold, respectively, after aerobic recovery. Transcript levels of miR-106b-5p increased by $1.6 \pm 0.07$-fold after anoxia treatment and remained high after aerobic recovery at $1.6 \pm 0.04$-fold compared to controls.

In response to dehydration/rehydration, all but one of the miRNAs evaluated in liver were downregulated during dehydration (Figure 2.9B). Transcript levels of miR-17$5 p$, miR-20-5p, and miR-25-3p decreased by $50 \pm 7.6 \%, 45 \pm 5.7 \%$, and $59 \pm 2.9 \%$, respectively, after dehydration compared to controls. Similarly, levels of miR-30a-5p and miR-30d-5p decreased by $53 \pm 6.8 \%$ and $46 \pm 7.9 \%$ respectively, in response to dehydration compared with controls. By contrast, the only response to rehydration was a $1.4 \pm 0.11$-fold increase in miR-34a-5p levels compared with controls. 
A comparable analysis of these ten miRNAs in skeletal muscle revealed only one significant change in response to anoxia, a $25 \pm 2.5 \%$ decrease in miR-20-5p in response to anoxia followed by a return to baseline after reoxygenation (Figure 2.10A). However, four miRNAs showed increased levels in response to dehydration/rehydration treatments (Figure 2.10B). Transcript levels of miR-25-3p increased by $1.9 \pm 0.1$-fold in response to dehydration and remained at this level $(1.9 \pm 0.3$-fold) after rehydration, as compared to the control group. MiR-30a-5p levels also rose by $1.9 \pm 0.2$-fold in response to dehydration but decreased again after rehydration. Transcript levels of both miR-34a-5p and miR-106b-5p increased by $1.4 \pm 0.08$-fold and $2.0 \pm 0.1$-fold, respectively, after dehydration and levels remained high at $1.6 \pm 0.07$-fold and by $1.7 \pm 0.22$-fold after rehydration compared to the control group.

\section{Relative transcript levels of 062}

Relative transcript abundance of $p 62$, a downstream target of TFEB and miR-17$5 p$ was assessed in liver over anoxia/reoxygenation and dehydration/rehydration cycles (Figure 2.11). Transcript levels of $p 62$ increased by $2.0 \pm 0.36$-fold after anoxia and by $2.2 \pm 0.3$-fold after dehydration treatment before returning to baseline after recovery in both conditions.

\section{Bioinformatic analysis of miR-17 binding site}

Previous studies have confirmed that miR-17-5p binds to the $p 62$ transcript to regulate its translation under different conditions [49,50]. Given that wood frogs are not genome sequenced, the full-length transcript of p62 (NM_001086451.2) from another frog, Xenopus laevis, was used to study the logistical probability of its binding with miR- 
17-5p (Figure 2.12). The predictions showed both seed-site and seedless-site binding in the 3'-UTR, 5'-UTR and the CDS of the $p 62$ transcript.

\subsection{Discussion}

Autophagy is a highly complex process whereby damaged macromolecules and organelles are tagged, degraded, and recycled in the cytoplasm. Studies have shown that autophagy is initiated in response to many stimuli including but not limited to organismal development [62], pathogenesis and disease [63], and during natural adaptation to environmental stresses [64,65]. While there are conflicting studies on the role of autophagy during stress [34,35], it is reasonable to assume that basal levels of autophagy could be beneficial to cells. Autophagy has been extensively studied during nutrient starvation [66]; however, wood frogs undergoing anoxia/reoxygenation and/or dehydration/rehydration cycles are metabolically depressed and have access to sufficient amounts of fermentable fuel (glucose, glycogen) as well as lipid fuels (in the case of dehydration). As such, it is unlikely that initiation of autophagy during stress in wood frogs is due to nutrient deprivation, but rather it could be a response that acts to clear up stress-induced damaged macromolecules and organelles from the cell.

Immunoblotting was used to assess the effects of anoxia/reoxygenation and dehydration/rehydration treatments on the expression levels of select proteins involved in autophagosome biogenesis. We hypothesized that energy expensive catabolic processes such as autophagy would be downregulated or maintained at a basal level under cell stress conditions to conserve energy under hypometabolic states. The results for liver show that total protein levels of ULK1 increased significantly in response to both anoxia 
and reoxygenation (Figure 2.2), and dehydration (Figure 2.3) before returning to normal upon rehydration. In agreement with these findings, previous studies have also reported an increase in protein levels of ULK1 following hypoxia treatment in different cells lines; suggesting that ULK1 activation may be necessary to initiate a similar stress-response in wood frogs $[67,68]$. ULK1 is regulated by reversible phosphorylation at two different sites: S555 activates and S758 inhibits. The increase in total ULK1 levels in the present study was accompanied by an increase in its active form (p-ULK1 S555) under anoxia Fig. 2.2), reoxygenation (Fig. 2.2), and dehydration in liver (Figure 2.3); similar findings were also observed in hypoxic cells [68]. Interestingly, although p-ULK1 phosphorylation at S555 is mediated by AMPK (AMP activated protein kinase) [69], an earlier study on wood frogs showed that the enzymatic activity of AMPK was not affected by anoxia or dehydration treatments in liver; and modest levels of AMPK remained active [70]. As such, perhaps the basal level of AMPK in liver was enough to phosphorylate ULK1 at S555 to promote its activation. Active ULK1 can then translocate to the isolation membrane (initiating from the endoplasmic reticulum, ER) and initiate the nucleation phase of autophagosome formation [71]. Activation of ULK1 by AMPK is balanced by deactivation of this kinase by mTORC1 (mechanistic target of rapamycin complex 1) to balance autophagic flux. Surprisingly, levels of the inactive form of ULK1 (phosphorylated at S758) also increased during anoxia, reoxygenation, dehydration and rehydration in the liver (Figure 2.2, 2.3). While information on mTORC1 responses to anoxia or dehydration stresses is not available for wood frogs, studies on other animal models capable of resisting various environmental stresses report either no change $[72,73]$ or an increase in mTOR activation following stress in liver [74]. Therefore, it is 
possible that an increase in p-ULK1 (S758) phosphorylation is caused by mTOR activation (or lack of inhibition) in wood frog liver. Interestingly, a recent study demonstrated that hyperosmotic stress also promotes ULK1 phosphorylation at S758 [75]. Given that dehydration in wood frogs not only increases the concentration of all ions and metabolites in cells, but also that frogs counter dehydration by a surge of synthesis of compatible solutes (glucose, urea) to help defend cell volume [9,17]. As such, it is possible that hyperosmotic conditions may be responsible in part for ULK1 inactivation. Hence, it appears that pools of both active and inactive ULK1 are present in liver, perhaps to easily promote or inhibit autophagosome biogenesis depending on the cellular requirements.

Nucleation and further maturation of phagophores involves an interconnected web of known and unknown proteins and complexes, that although have similar functions, are involved in different mechanisms of autophagy induction. A simplistic paradigm of phagophore nucleation depends on ULK1 activation, where active ULK1 in a complex with ATG13, FIP200 (FAK family interacting protein 200) and ATG101 promotes the recruitment of the nucleation complex containing many proteins including beclin1 and ATG14, to the growing phagophore [76,77]. The PtdIns 3K (phosphoinositide-3-kinase) is involved in two complexes, where complex I involving VPS15 (vacuolar protein sorting 15), VPS34, ATG14 and beclin1 regulates autophagy and complex II (VPS15, VPS34, VPS38 and beclin1) regulates vacuolar protein sorting; for review see [78]. The coiled-coil domain of ATG14 interacts with beclin1, and this interaction is necessary for the recruitment of the VPS34 complex to the autophagosome biogenesis site at the ER [79]. This recruitment facilitates the production and incorporation of PI3P 
(phosphatidylinositol 3-phosphate) to the ER membrane, thereby promoting the formation of membrane curvature termed the omegasome (nucleation step) [80]. Beclin1 is another crucial regulator of autophagy, and it contains three domains: a Bcl-2 homology (BH)-3 domain [81], a coiled-coil domain (CCD) [82], and an evolutionarily conserved domain (ECD)[83], where each domain interacts with a set of co-factors to maintain homeostasis between autophagy and apoptosis in the cell; for review see [84]. For example, under normal conditions, the $\mathrm{BH} 3$ domain of beclin1 can physically interact with the anti-apoptotic Bcl-2 and Bcl-XL proteins to keep both processes at homeostatic levels [85]. This interaction also leads to beclin1 homodimerization and inactivation which results in the inhibition of autophagosome biogenesis [86]. There are multiple mechanisms that can induce beclin1 activation and the initiation of autophagosome biogenesis, and while they are different, the outcome is the same. Firstly, phosphorylation of Bcl2 by JNK1 (c-jun N-terminal kinase1) can free beclin1 from its homodimerized state, allowing it to heterodimerize with ATG14 to induce autophagosome biogenesis [87]. Secondly, phosphorylation by kinases such as ULK1 at S30 [88] and or AMPK at S93 [89] can also lead to beclin1 activation, its association with ATG14 [89] and the induction of phagophore nucleation. Thirdly, when cells are faced with oxidative stress, a non-histone chromatin binding protein called the high mobility group box 1 (HMGB1) is translocated to the cytoplasm [90]. HMGB1 shares considerable homology with Bcl2 but has higher affinity for beclin1 [91]. As such, it competes with Bcl2 for beclin1, and its binding frees Bcl2 from beclin1 in favor of inhibiting apoptosis and promoting autophagy [91,92]. Overall, the underlying mechanism is that liberated beclin1 will bind to ATG14 and promote the nucleation of the autophagosome. In the present study, levels of ATG14 
were unaffected by anoxia/reoxygenation and dehydration, but levels decreased significantly during rehydration in liver (Figure 2.2, 2.3). A lack of positive response from ATG14 under such conditions may suggest that autophagosome formation is unaffected in liver of wood frogs exposed to anoxia/reoxygenation and dehydration, whereas further attenuation in autophagosome formation occurs after rehydration. Interestingly, it was reported that ATG14, a crucial regulator of autophagy, is recycled back to the cytoplasm after autophagosome maturation following fusion with the lysosome [93]. Given that mRNA translation is greatly suppressed during MRD, it is energetically more beneficial to increase the stability of proteins by extending protein half-life via PTMs [94]. Therefore, despite a lack of positive response during these conditions, if we suppose that only basal levels of autophagy are retained in the cell, then the present amount of recyclable ATG14 could in theory be enough to facilitate the autophagosome biogenesis process, albeit at lower levels. Total levels of beclin1 decrease under anoxia, returned to baseline after reoxygenation, and were unchanged in response to dehydration/rehydration treatment in liver (Figure 2.2, 2.3). Interestingly, the content of p-beclin1 (S93), the active form, remained unaffected after anoxia/reoxygenation but increased significantly during dehydration before returning to baseline after rehydration in the same tissue (Figure 2.2, 2.3). A decrease in total beclin1 levels during anoxia followed by a lack of response by its active form indicates that perhaps autophagosome initiation is decreased below basal level during anoxia but returns to baseline after reoxygenation. Conversely, a significant increase in the active form of p-beclin1 in dehydrated liver hints at a potential increase in autophagosome biogenesis in this tissue, whereas this response returns to baseline after rehydration. Phosphorylation of p-beclin1 
(S93) by AMPK [89] promotes its association with ATG14 [89], and the recruitment of more complexes to the growing phagophore. Moreover, the increase in active p-ULK1 (p-ULK S555) seen during dehydration (Figure 2.3) could also promote the phosphorylation of beclin1 at an alternative site (S30) to stabilize its interaction with ATG14 [88], and phosphorylation of ATG14 at S29 to promote the PI3P production activity of VPS34 [95].

Binding of ATG14 to PI3P on the omegasome enhances the membrane sensing ability of ATG14, which stabilizes the growing curvature and causes the recruitment of the autophagosome elongation complex (ATG5, ATG12 and ATG16) [80]. The ubiquitin activating enzyme E1-like ATG7 and the ubiquitin conjugating E2-like ATG10 covalently attach ATG5 to the ubiquitin-like ATG12 proteins [96]. The constitutive binding of ATG5 and ATG12 is irrespective of the autophagic state of the cell and together, these proteins are translocated to the isolation membrane upon autophagic stimuli [97]. The ATG5-ATG12 complex can also bind non-covalently with ATG16, a multimeric protein that is crucial for directing the localization of the elongation complex to the growing isolation membrane [97,98]. It was shown that ATG5 is crucial for membrane targeting and phagophore maturation [98], and deletion of ATG5 results in decreased autophagic vesicles in embryonic stem cells [96]. The current data showed no significant change in ATG5 levels over the anoxia/reoxygenation cycle in liver, but surprisingly, levels decreased during dehydration before returning to normal after rehydration (Figure 2.2, 2.3). A lack of response during anoxia could, again, be indicative of basal levels of autophagosome formation; however, a decrease during dehydration would suggest a reduction in this process. The latter is inconsistent with the remaining 
markers that suggest a potential increase in autophagosome formation. During phagophore maturation, ATG5 is localized to the outer membrane of the growing autophagosome [96]. Indeed, the asymmetric distribution of ATG5 allows the ATG5-12 complex to dissociate from the fully formed autophagosome and before its fusion with the lysosome, suggesting that perhaps this complex is also recyclable [96]. The recycling of proteins rather than their degradation and de novo synthesis is a hallmark of MRD as it allows cells to conserve the finite amount of energy available while still maintaining basal activity levels. Alternatively, a decrease in ATG5 during dehydration may be in response to limiting its non-autophagic activities. A previous study reported that cleavage of ATG5 by calpain generates a truncated form of ATG5 that can translocated to the mitochondria and binds with the anti-apoptotic Bcl-XL protein [99]. This association activates Bax and promotes the release of cytochrome $\mathrm{c}$ from the mitochondria resulting in cell death [99]. In wood frogs, anoxia and reoxygenation treatments induced the expression of numerous anti-apoptotic proteins in liver, including Bcl-XL, which in summation demonstrate a strong inhibition of apoptosis under such conditions [23]. Therefore, the limited amount of ATG5 available under the same conditions is probably only allocated to promoting autophagosome assembly and maturation. In contrast, the decrease in protein levels of ATG5 during dehydration could be a response to limit the initiation of apoptosis in liver and channel the limited ATG5 available only toward autophagosome maturation.

Another step necessary for autophagosome maturation and selective autophagy is the attachment of numerous adaptor proteins, including but not limited to LC3 and p62. The LC3 family is composed of multiple proteins (including but LC3A, LC3B and 
LC3C), but LC3B has been extensively used as an autophagy marker $[100,101]$. The ATG12 component of the ATG5-ATG12-ATG16 complex (resembles an E3 enzyme) physically interacts with ATG3, facilitates its E2-like activity, and has been shown to be crucial for the recruitment of LC3 to the growing autophagosome [102]. Pro-LC3B is cleaved by ATG4 to generate LC3I [103]. This cleavage exposes the C-terminal glycine residue of the protein, facilitating the E1-like ATG7, the E2-like ATG3, and the E3-like ATG5-ATG12-ATG16 complex-mediated conjugation of phosphatidylethanolamine (PE) to LC3BI to generate LC3BII $[101,102]$. Lipidated LC3B (LC3BII) can then be localized both on the inside and outside of the autophagosomal membrane [104], and this localization will promote the recruitment of $\mathrm{p} 62$ to the maturing autophagosome [105]. The p62 protein serves as an adaptor molecule that enables the recruitment and engulfment of the polyubiquitinated macromolecules by the maturing autophagosome and their degradation later in the autolysosome [106]. Once the maturation of the autophagosome is complete, ATG14 can also directly bind many proteins, including the SNARE (SNAP receptor) proteins STX17-SNAP29, found on the autophagosome and promote their fusion with VAMP8 (vesicle-associated membrane protein 8) proteins found on the lysosome, to form the autolysosome [93,107]. It was postulated that dephosphorylation of PI3P leads to the dissociation of the majority of ATG14 proteins from the autophagosome to promote curvature instability, a process that will prime the autophagosomal membrane for fusion with the lysosome [80]. Interestingly, the remaining ATG14 along with STX17 proteins can be retrieved from the autolysosome and recycled back into the cytoplasm for reuse [93]. 
The present results show that levels of ATG3 and ATG4a both decreased significantly in response to anoxia and reoxygenation, whereas levels of p62 and LC3BI increased significantly after anoxia treatment liver in (Figure 2.2). Levels of LC3BI remained high after reoxygenation in the same tissue (Figure 2.2). Interestingly, correlated with significantly low levels of ATG3 and ATG4a, immunoblotting failed to detect any LC3BII under the same conditions, suggesting that perhaps the few autophagosome that do form do not fuse with the lysosome to complete the autophagic degradation process. Indeed, an LC3BII/LC3BI ratio of higher than 1 is indicative of autolysosome formation and is considered one of the gold-standard methods for assessing autophagic flux in the cell $[108,109]$. Lack of fusion of the autophagosome with the lysosome during anoxia and reoxygenation in wood frog liver is also consistent with the lack of p62 degradation (Figure 2.2). Therefore, given that p62 is degraded with the tagged cargo in the autolysosome and can be used as a marker to measure autophagic flux $[108,109]$, then an increase in the levels of both p62 and LC3BI could suggest a strong attenuation in autophagosome fusion with the lysosome in following anoxia/reoxygenation treatments. Overall, it appears that cells exposed to these conditions form limited numbers of autophagosomes to capture damaged macromolecules and organelles but delay fusion with lysosomes and cargo degradation until such time as energy levels are high again.

After the dehydration/rehydration treatments, liver ATG3 and ATG4 levels remained constant (Figure 2.3). The data also showed a significant increase in both p62 and LC3BII/I ratio after dehydration treatment in liver before returning to normal after rehydration (Figure 2.3). While levels of the two latter markers increased, the proteins 
that regulate their attachment to the autophagosome remained the same. This is not unusual if low levels of autophagic flux are assumed. Moreover, many proteins of the autophagosome biogenesis machinery, including ATG3 and ATG4, will dissociate from the mature autophagosome before its fusion with the lysosome and are recycled back to the cytoplasm for subsequent conjugations [110]. As such, despite a lack of increase in these levels, it is still possible to have a basal level of autophagic degradation. The increase in LC3BII levels indicates the formation of complete autolysosomes and potentially an increase in autophagic flux, however an increase in p62 levels can also indicate a lack of autophagic degradation. While these results are somewhat conflicting, it is likely that some of the mature autophagosomes fuse with lysosomes during dehydration to degrade the tagged cargo. While dehydration can reduce the frog's metabolic rate substantially, these organisms still have access to oxygen albeit at hypoxic levels. Therefore, in comparison to anoxia where no oxygen is available, dehydration appears to favor the formation of more autolysosomes in support of increasing autophagic flux and cargo degradation under these conditions.

Autophagy can also be regulated at the transcriptional level by tweaking the transcriptional activity of various transcription factors necessary for transcribing proteins involved in the initiation, nucleation, elongation and maturation complexes [111]. Of the many transcription factors involved, this study focused on TFEB that is a master regulator of lysosomal biogenesis and has been shown to regulate various proteins involved in autophagosome biogenesis [39,41,42,112]. Under normal conditions, TFEB is heavily phosphorylated by multiple kinases (mTORC1, ERK2, etc.) and is sequestered in the cytoplasm where it associates with protein 14-3-3 [42,113]. Once the 
transcriptional activity of TFEB is required, calcineurin facilitates TFEB dephosphorylation, thereby allowing it to dissociate from protein 14-3-3 and enter the nucleus [113]. In the nucleus, TFEB binds to many autophagy and lysosomal genes containing the CLEAR element to induce their expression $[39,41,42,112]$. Of interest is the localization of TFEB. This transcription factor can be found in the cytoplasm as well as on the lysosomal surface where it interacts with the LYNUS (lysosome nutrient sensing) machinery, and is either active or inactive depending on the cellular nutrient levels [112]. Given the importance of TFEB in regulating both autophagy and lysosomal biogenesis, an examination of its transcriptional activity under anoxia/reoxygenation and dehydration/rehydration conditions was justified.

Analysis of the DNA binding activity of TFEB to its consensus sequence used DPI-ELISA. The results show that the DNA binding ability of TFEB increased significantly under anoxia before returning to baseline after aerobic recovery in liver (Figure 2.6A). Interestingly, the total protein levels of TFEB remained the same over the anoxia/reoxygenation treatment in the same tissue (Figure 2.7A). This suggests that probable regulation of TFEB at the posttranslational level, instead of its de novo synthesis, is responsible the increase in DNA binding activity. Indeed, TFEB is dephosphorylated in response to various stimuli by calcineurin, and that step is required for its nuclear localization and enhanced DNA binding activity [113]. A previous study from our lab showed an increase in calcineurin levels after anoxia treatment in liver of wood frogs [56]; suggesting that perhaps calcineurin is dephosphorylating and activating TFEB in this tissue. On the other hand, the present results also demonstrate an increase in TFEB DNA binding, and total protein levels in response to dehydration, with levels 
returned to baseline after rehydration (Figure 2.6B, 2.7B). This suggests that perhaps dehydrated cells require a higher number of lysosomes, a finding that could be supported by a potential increase in autophagic flux in liver during dehydration.

TFEB has been shown to directly bind to the promoters of autophagy-related genes including but not limited to ATG4, LC3B, beclin1, p62 and the lysosomal hydrolase ATP6V0D1 (ATPase $\mathrm{H}+$ transporting V0 subunit D1) [41,111,113]. Correlation with the present data, the increase in TFEB DNA binding in liver extracts from anoxic and dehydrated correlates with an increase in p62 and LC3BI levels in the tissue (Figure 2.2, 2.3), but not with the remaining factors. Moreover, levels of ATP6V0D1 remained unchanged during anoxia and dehydration, despite an increase in TFEB DNA binding levels, and decreased further during reoxygenation and recovery in the same tissue (Figure 2.7A, 2.B). Perhaps maintaining only a basal level of this lysosomal hydrolase is necessary during stress in wood frogs. This hypothesis also fits with the proposed potential levels of autophagic flux under these conditions. It is likely that due to limited energy available, degradation of the tagged cargo occurs at later stages of recovery when full aerobic respiration is restored.

Regardless, the differential regulation of autophagy and lysosomal biogenesis genes by TFEB suggests the involvement of a multi-level surveillance system that regulates both processes. Therefore, it is possible that TFEB-mediated gene expression is regulated either upstream of transcription by epigenetic factors that render specific genes inaccessible to TFEB, or at the posttranscriptional level by miRNAs. To determine whether the expression of autophagy targets is regulated at the posttranscriptional level, a 
list of miRNAs that are known to regulate autophagy was curated from the literature. Interestingly, whereas the general pattern of miRNA expression correlates well with the dehydration/rehydration data, the correlation between the same miRNAs and their downstream targets is poor under anoxia/reoxygenation conditions, suggesting that perhaps other modes of regulation may be involved. For example, miRDB (http://www.mirdb.org/) predicted 662 potential mRNA transcripts, including ULK1, that harbored the conserved sequence for mature miR-25-3p. This prediction was also validated by another group, where inhibition or overexpression of miR-25-3p increased and decreased levels of ULK1 proteins, respectively [114]. Whereas these results show a significant increase in miR-25-3p levels during reoxygenation (Figure 2.9A), protein levels of ULK1 remain significantly high under the same condition (Figure 2.2). Given the complexity of anoxia tolerance and autophagy, it is possible that induction of miR-25$3 p$ during reoxygenation is due to its alternative role in regulating other non-autophagy targets. The results also showed an increase in miR-26-5p after both anoxia and reoxygenation treatments (Figure 2.9A). This miRNA was shown to bind to the 3'UTR of ULK2 transcripts in cancer cells leading to ULK2 transcript suppression and the inhibition of autophagy [115]. ULK1 and ULK2 are both functionally redundant, and therefore activation of autophagy could proceed even if only one of these kinases is available. In the present study the levels of ULK1 were significantly increased over the anoxia/reoxygenation treatment. As such, it is possible that an increase in miR-26-5p suppresses the expression of ULK2 under the same conditions to conserve energy during MRD. The present results also show an increase in the levels of miR-30a-5p and miR30d-5p after reoxygenation (Figure 2.9A). Previous studies have shown that miR-30a-5p 
binds to the 3'UTR of the beclin1 transcript [116,117], whereas miR-30d-5p overexpression was shown to reduce protein levels of ATG2B, ATG5, ATG12, beclin1 and BNIP3L, resulting in the reduction in the LC3BI to LC3BII conversion [118]. While levels of ATG5 and beclin1 were not affected by overexpression of miR-30d-5p or miR$30 \mathrm{a}-5 \mathrm{p}$ in the case of beclin1 upon reoxygenation, detection of LC3BII did not occur under the same condition despite an increase in LC3BI levels (Figure 2.2). Although further evaluation is warranted, it is possible that an increase in these microRNAs is responsible for the absence of LCBI lipidation observed after reoxygenation, possibly as an effort to reduce autophagic flux under this condition.

Levels of miR-106b-5p increased over the anoxia/reoxygenation cycle (Figure 2.9A). Previously, it was found that overexpression of miR-106b-5p was shown to downregulate ATG16L1 protein expression by targeting its transcript at the 3'UTR, and reducing the conversion of LC3BI to LC3BII [119]. Interestingly, the same study also demonstrated that transfection with miR-106b-5p inhibited ATG12 gene expression, only minimally reduced beclin 1 gene expression, and surprisingly caused an increase in p62 protein levels [119]. Given that the current study shows the absence of LC3BII, a minimal decrease in beclin 1 and a significant increase in p62 levels, it is possible that overexpression of miR-106-5p was associated with these changes during anoxia. Thus, with the complexity of autophagy, the versatility of miRNAs, and the nature of the stress, it appears that multiple networks of miRNAs regulate the expression of autophagy proteins simultaneously. Regardless, these findings demonstrate the precision and rigor of controls involved in fine-tuning this process over the anoxia/reoxygenation cycle. 
The same analysis was performed for liver from dehydrated/rehydrated frogs. The trend of miRNA expression following dehydration/rehydration treatments correlates well with the liver protein expression data under the same conditions. For example, overexpression of miR-20-5p and miR-25-3p was shown to inhibit ULK1 protein expression $[114,120]$. The current study shows the opposite result - a decrease in transcript levels of both miRNAs (Figure 2.9B) correlated with an increase in ULK1 protein levels during dehydration (Figure 2.3). Therefore, upregulation of ULK1 may be facilitated by the actions of these two miRNAs during dehydration. Moreover, levels of miR-30a-5p and miR-30d-5p were significantly reduced during dehydration (Figure 2.9B) suggesting that lower levels of miR-30a-5p could help to stabilize the protein levels of its downstream target, beclin1, that did not change following dehydration treatment (Figure 2.3). Perhaps, given that autophagy appears to be more active in liver of dehydrated frogs, it is possible that alternative methods of regulation are involved. Indeed, we have demonstrated that beclin1 levels are regulated by phosphorylation and that an increase in p-beclin1(S93) could be responsible for promoting autophagosome maturation (Figure 2.3). It is possible that a decrease in miR-30d-5p levels during dehydration was responsible for the enhanced expression of ATG2B, ATG12 and BNIP3L in wood frog liver. The upregulation of these proteins promotes conversion of LC3BI to LC3BII, a finding that was evident in the results following dehydration/rehydration treatments (Figure 2.3). MiR-40d-5p was also reported to target ATG5 and beclin1 [118]. Surprisingly, despite evidence of some autophagic flux during dehydration, levels of ATG5 were reduced and beclin1 remained the same in liver (Figure 2.3). As such, it is possible that ATG5 and beclin1 levels are regulated by 
alternative mechanisms that enhance their enzymatic activity and protein stability. The present results also demonstrate an increase in miR-34a-5p (Figure 2.9B) during rehydration, and this miRNA was shown to target the 3'UTR of the HMGB1 transcript [121]. Stress-induced cytosolic translocation of HMGB1 displaces Bcl2 from the beclin1Bcl2 complex, thereby allowing beclin1 to participate in autophagosome biogenesis and Bcl2 to inhibit apoptosis [91,92]. Therefore, it is possible that induction of miR-34a-5p contributes to reducing HMGB1 levels to help dampen autophagic flux during rehydration. Further experiments are warranted.

Since the genome of wood frogs have not been sequenced, validation of all gene transcript levels could not be done to support the miRNA results. However, transcript levels of p62 were measured in response to anoxia/reoxygenation and dehydration/ rehydration in liver (Figure 2.11). The results show that both anoxia and dehydration stresses significantly increased p62 transcript levels, but values returned to normal during recovery. Correlated with these results, the protein levels of p62 also increased under the same conditions (Figure 2.2, 2.3). Previous studies have demonstrated that overexpression miR-17 reduces the protein levels of p62 in different cell lines and under different conditions $[49,50]$. The results of the present work show that whereas transcript levels of miR-17-5p show a decreasing trend in response to anoxia and reoxygenation, their difference is not statistically significant (Figure 2.9A). However, in response to dehydration, transcript levels of miR-17-5p decreased significantly but returned to baseline following rehydration (Figure 2.9B). These changes during anoxia and dehydration may be responsible for promoting p62 protein translation in an effort to support autophagosome assembly. Indeed, bioinformatic analysis predicted both seed 
binding and seedless regions in the 3'UTR, 5'UTR and CDS of p62 transcripts where miR-17-5p could bind (Figure 2.12). Therefore, it is highly likely that the expression of miR-17-5p could contribute in part to regulating the expression of p62 protein under stress conditions in wood frog liver. Altogether, it is evident that miRNAs, at least in part, play a role in regulating autophagic flux in response to anoxia/reoxygenation and dehydration/rehydration in wood frog liver.

To study whether the regulation of autophagosome biosynthesis occurs in a tissuespecific manner, the same analyses were carried out on skeletal muscle from wood frogs that underwent anoxia/reoxygenation or dehydration/rehydration treatments. Both liver and muscle contain glycogen stores; however, only liver glycogen is used to generate glucose to be distributed to the other organs. Muscle glycogen is allocated only to muscle-specific use and does not contribute to overall cryoprotection for other organs. As such, this raised interest in comparing autophagy and lysosomal biogenesis in a metabolically active tissue such as liver with a tissue that is less metabolically active under environmental stress conditions such as skeletal muscle.

In contrast to the liver, many of the targets involved in autophagosome biogenesis are significantly downregulated after anoxia/reoxygenation and dehydration/rehydration in wood frog muscle (Figure 2.4, 2.5). The results show a significant decrease in components of the nucleation complex; where levels of ULK1, beclin1, and ATG14 decreased significantly in response to anoxia and reoxygenation in muscle (Figure 2.4). Furthermore, there was no change in the phosphorylation levels of the active form of $\mathrm{p}$ ULK1 (S555) following anoxia/reoxygenation or dehydration/rehydration treatments 
(Figure $2.4,2.5$ ). The activity of AMPK, the kinase that mediates the phosphorylation on ULK1, was not affected by anoxia treatment but increased significantly during dehydration in skeletal muscle [70]. Given the extremely low protein levels of ULK1 following anoxia/reoxygenation, the basal level of AMPK available may be responsible for maintaining its S555 phosphorylation status (Figure 2.4). However, despite an increase in AMPK levels during dehydration, levels of p-ULK1 S555 remained unchanged under the same condition in the muscle (Figure 2.5). This suggests that perhaps a basal level of autophagosome formation is occurring in the muscle; however, given that total ULK1 levels were minuscule, the number of autophagosomes formed must be low.

Recruitment of ATG13, FIP200 and ATG101 following ULK1 activation facilitates the recruitment of beclin1 and ATG14, two factors that are crucial for autophagosome biogenesis [76,77]. The results for wood frog muscle show a decrease in beclin1 and ATG14 levels following anoxia and reoxygenation, but levels of both targets remained unchanged in response to dehydration and rehydration treatments (Figure 2.4, 2.5). A significant decrease in beclin1 and ATG14 strongly supports attenuation of autophagosome nucleation following anoxia/reoxygenation treatments, but a basal level of autophagosome initiation would be maintained following dehydration/rehydration treatment. As mentioned earlier, recruitment of the ATG5-ATG12-ATG16 complex to the growing autophagosome is required for the maturation of the autophagosome and the recruitment of other adaptor proteins that are crucial of cargo targeting and autophagosome closure $[97,98,102]$. Our results demonstrate a significant reduction in ATG5 protein levels following anoxia, reoxygenation, dehydration and rehydration 
treatments in wood frog muscle, again suggesting a strong reduction in autophagosome biogenesis. Although ATG5 has been shown to be recycled in the cell [96], in combination with other factors, a strong case against autophagosome maturation can be made.

As the autophagosome matures, ATG4 cleaves pro-LC3B to generate LC3BI [103], and this facilitates the activity of ATG7, ATG3 and the ATG5-ATG12-ATG16 complex, whereby PE is attached to LC3BI to form LC3BII [101,102]. As a hallmark of autophagosome maturation, LC3BII then coats both the outside and the inside of the autophagosome [104] before recruiting the p62 adaptor protein to promote selective cargo engulfment [106]. The results for wood frog muscle show a significant decrease in ATG4 levels following anoxia, reoxygenation and dehydration before returning to normal upon rehydration (Figure 2.4, 2.5). Interestingly, the changes in ATG4 levels following anoxia, reoxygenation, and rehydration did not appear to affect its downstream activity in cleaving LC3B into LC3BI under the same conditions, although levels rose following the respective recoveries (Figure 2.4, 2.5). Levels of ATG3, one of the E2-like enzymes necessary for LC3BII conversion were significantly downregulated following anoxia but were unchanged upon reoxygenation, dehydration or rehydration. The decrease or lack of response by ATG3 was evident, since under all conditions skeletal muscle failed to convert LC3BI into LC3BII. Together, these results suggest the absence or a very limited number of autophagosomes formed under all conditions. Interestingly, whereas low levels of p62 indicated an increase in cargo recognition and engulfment, autophagosome fusion with the lysosome and increase in autophagic flux, without other markers, these results could be misleading [106]. Levels of p62 decreased strongly under anoxia and 
reoxygenation, as compared with controls, but remained unaffected following dehydration and rehydration (Figure $2.4,2.5$ ). Low levels of p62, in combination with our previous findings, suggest that muscle exposed to anoxia and reoxygenation, and to a lesser degree during dehydration and rehydration treatments, significantly reduces the process of autophagosome formation, leading to incomplete autophagy. As explained earlier, under energy-restricted conditions, ATP-expensive processes such as autophagy are suppressed in an effort to conserve energy for pro-survival processes. Therefore, wood frogs strategically use checkpoints at multiple biological levels to maintain the autophagic machinery at bay under high stress conditions that might otherwise trigger this catabolic process.

As explained earlier, TFEB is a master regulator of lysosomal biogenesis and has been shown to also regulate the expression of several genes involved in autophagosome biogenesis $[39,41,42,112]$. The results of this study showed that the DNA binding activity of TFEB was significantly reduced following anoxia and reoxygenation, remained unaffected during dehydration but decreased significantly following rehydration (Figure 2.6A, 2.6B). Given that this transcription factor regulates the vast majority of genes involved in both autophagy and lysosome formation, the data on TFEB binding to DNA are consistent with a decrease in autophagic flux. The reduction in DNA binding could also be due to a significant decrease in TFEB protein levels following anoxia/reoxygenation treatments (Figure 2.8A). Interestingly, the same correlation was seen for dehydration/rehydration treatments, where unchanged DNA binding (Figure 2.6B) was associated with unchanged protein levels of TFEB following dehydration, before significantly decreasing after rehydration (Figure 2.8B). Thus, these results for 
TFEB protein levels strongly suggest an attenuation of the autophagy and lysosomal biogenesis responses at the transcriptional level. Indeed, transcriptional regulation is one of the main controlling steps involved in MRD [13,27,122].

Downregulation of targets involved in autophagosome biogenesis is also supported by the results for TFEB analysis. Studies have shown that TFEB regulates the transcription of numerous autophagy genes including but not limited to ATG4, beclin1, p62 and ATP6V0D1 [41,111,113]. In response to anoxia, expression of ATG4, beclin1, p62 and ATP6V0D1 in wood frog skeletal muscle (Figure 2.4, Figure 2.8A) correlated well with TFEB DNA binding in response to anoxia, thereby providing another line of evidence that both components of the autophagosome machinery and lysosomal biogenesis are significantly reduced in muscle under stress conditions. Results for dehydrated/rehydrated muscle also showed a correlation between unchanged DNA binding activity of TFEB following dehydration, and the protein levels of Beclin1, p62, and ATP6V0D1 (Figure 2.5, Figure 2.8B). Altogether, these results suggest that transcriptional regulation, mediated in part by TFEB, is responsible for a significant reduction in the levels of proteins involved in autophagosome and lysosomal biogenesis, resulting in a decrease in autophagic flux in muscle.

Finally, we also examined the involvement of miRNAs in regulating protein synthesis under stress and recovery in muscle. Whereas many of the miRNAs measured showed downregulation in response to anoxia, only one miRNA showed a statistically significant decrease. MiR-20 is previously shown to suppress the expression of ULK1 proteins [120], and therefore a reduction in its levels (Figure 2.10A) should lead to an 
increase in ULK1 protein levels. Interestingly, our analysis shows a significant reduction in ULK1 levels; suggesting that perhaps the expression of this kinase is regulated by other factors upstream of miRNA control. Indeed, a reduction in TFEB activity could account for this change and supports the proposal that ULK1 expression is regulated at the transcriptional level during anoxia/reoxygenation in wood frog skeletal muscle.

Most of the miRNAs measured in muscle showed a significant increase following dehydration and rehydration treatments, and our results demonstrate a strong correlation between levels of the miRNA and their downstream targets. For example, levels of miR25-3p increased significantly in response to dehydration and rehydration (Figure 2.10B), which correlates well with the decreased protein levels of ULK1 under the same conditions (Figure 2.5A). Indeed, it was previously shown that overexpression of miR25-3p resulted in reduced levels of ULK1 protein [114]. As such, it is likely that the reduction of ULK1 protein levels seen in wood frog muscle during dehydration and rehydration is mediated post-transcriptionally via miRNAs. Other miRNAs such as miR30a 5p increased following dehydration, whereas miRNA 34a-5p and miR-106b-5p were elevated under both dehydration and rehydration treatments (Figure 2.10B). Previously we correlated the expression of miR30a with regulation of beclin1 [116,117], and while this remains a possibility, our results for the response to dehydration indicate otherwise. The increase in miR30a-5p (Figure 2.10B) did not affect the protein levels of beclin1 (Figure 2.5) following either dehydration or rehydration treatments, but a strong reduction in ATG5 protein levels occurred under the same conditions (Figure 2.5B). Considering that ATG5 plays a significant role in autophagosome maturation, reduced levels of this protein, in part mediated at the posttranscriptional level, could account for 
reduced autophagosome biogenesis in this tissue. Moreover, it was previously hypothesized that induction of miR-34a-5p could be responsible for reducing the expression of HMGB1, leading to promotion of autophagosome formation [121]. Although levels of HMGB1 in muscle were not measured, an increase in this miRNA could suggest a decrease in HMGB1 expression levels. As such, this too could decrease autophagosome biogenesis by retaining the inactive form of beclin1 in the cytoplasm. Lastly, the increase in miR-106b-5p seen in response to dehydration/rehydration could result in the downregulation of ATG16 [119]. Decreased levels of ATG16 could lead to an inhibition of the conversion of LC3BI to LC3BII [119], and this was evident in the results since LC3BII was not detected in any of the muscle samples (Figure 2.5). Altogether, these data demonstrate that miRNAs could be significant contributors to regulating autophagosome biosynthesis in response to dehydration/rehydration in wood frog muscle.

In conclusion, our results demonstrate a strong decrease in autolysosome formation in response to anoxia/reoxygenation in liver and muscle, a decrease in autolysosome formation in response to dehydration/rehydration in muscle, and a potential maintenance (or perhaps a small increase) of formation of autolysosomes in liver post dehydration/rehydration. The data also show that autophagosome and lysosomal biogenesis are regulated at all biological levels (transcript, post-transcription and posttranslation), and that perfect regulation of autophagic flux depends on perfect synchrony between all these regulatory check points. 
Acknowledgements: We thank J.M. Storey for editorial review of this manuscript. This work was supposed by a Discovery Grant from the Natural Sciences and Engineering Research Council of Canada (Grant \#6793). K.B.S. holds the Canadian Research Chair in Molecular Physiology and R.A. holds an Ontario Graduate Scholarship. 


\subsection{References- Chapter 2}

[1] W.D. Schmid, Survival of frogs in low temperature., Science. 215 (1982) 697-8. https://doi.org/10.1126/science.7058335.

[2] K.B. Storey, J.M. Storey, Natural freeze tolerance in ectothermic vertebrates., Annu. Rev. Physiol. 54 (1992) 619-37. https://doi.org/10.1146/annurev.ph.54.030192.003155.

[3] J.R. Layne Jr., R.E. Lee Jr., J.L. Huang, Inoculation triggers freezing at high subzero temperatures in a freeze-tolerant frog (Rana sylvatica) and insect (Eurosta solidaginis), Can. J. Zool. 68 (1990) 506-510. https://doi.org/10.1139/z90-074.

[4] J.P. Costanzo, J.M. Bayuk, R.E. Lee, Inoculative freezing by environmental ice nuclei in the freeze-tolerant wood frog, Rana sylvatica, J. Exp. Zool. 284 (1999) 7-14. https://doi.org/10.1002/(SICI)1097-010X(19990615)284:1<7::AIDJEZ2>3.0.CO;2-4.

[5] B. Rubinsky, S.T. Wong, J.S. Hong, J. Gilbert, M. Roos, K.B. Storey, 1H magnetic resonance imaging of freezing and thawing in freeze-tolerant frogs., Am. J. Physiol. 266 (1994) R1771-7. https://doi.org/10.1152/ajpregu.1994.266.6.R1771.

[6] K.B. Storey, Glycolysis and the regulation of cryoprotectant synthesis in liver of the freeze tolerant wood frog, J. Comp. Physiol. B. 157 (1987) 373-380. https://doi.org/10.1007/BF00693364.

[7] K.B. Storey, J.M. Storey, Biochemical adaption for freezing tolerance in the wood frog, Rana sylvatica, J. Comp. Physiol. B. 155 (1984) 29-36. https://doi.org/10.1007/BF00688788.

[8] J.P. Costanzo, R.E. Lee, Avoidance and tolerance of freezing in ectothermic vertebrates, J. Exp. Biol. 216 (2013) 1961-1967. https://doi.org/10.1242/jeb.070268.

[9] T.A. Churchill, K.B. Storey, Dehydration tolerance in wood frogs: A new perspective on development of amphibian freeze tolerance, Am. J. Physiol. Integr. Comp. Physiol. 265 (1993) R1324-R1332. https://doi.org/10.1152/ajpregu.1993.265.6.R1324.

[10] J.R. Layne, R.E. Lee, T.L. Heil, Freezing-induced changes in the heart rate of wood frogs (Rana sylvatica)., Am. J. Physiol. 257 (1989) R1046-9. https://doi.org/10.1152/ajpregu.1989.257.5.R1046.

[11] R. Al-attar, Y. Zhang, K.B. Storey, Osmolyte regulation by TonEBP/NFAT5 during anoxia-recovery and dehydration-rehydration stresses in the freeze-tolerant wood frog (Rana sylvatica), PeerJ. 5 (2017) e2797. https://doi.org/10.7717/peerj.2797.

[12] C.P. Holden, K.B. Storey, Second messenger and cAMP-dependent protein kinase responses to dehydration and anoxia stresses in frogs., J. Comp. Physiol. B. 167 (1997) 305-12. https://doi.org/10.1007/s003600050078.

[13] J. Zhang, L.J. Hawkins, K.B. Storey, DNA methylation and regulation of DNA 
methyltransferases in a freeze-tolerant vertebrate, Biochem. Cell Biol. (2019) 1-9. https://doi.org/10.1139/bcb-2019-0091.

[14] K.B. Storey, Life in the slow lane: Molecular mechanisms of estivation., Comp. Biochem. Physiol. A. Mol. Integr. Physiol. 133 (2002) 733-54. https://doi.org/10.1016/S1095-6433(02)00206-4.

[15] J.P. Costanzo, M.C.F. do Amaral, A.J. Rosendale, R.E. Lee, Hibernation physiology, freezing adaptation and extreme freeze tolerance in a northern population of the wood frog., J. Exp. Biol. (2013). https://doi.org/10.1242/jeb.089342.

[16] J.P. Costanzo, R.E. Lee, Urea loading enhances freezing survival and postfreeze recovery in a terrestrially hibernating frog, J. Exp. Biol. 211 (2008) 2969-2975. https://doi.org/10.1242/jeb.019695.

[17] J.P. Costanzo, A.M. Reynolds, M.C.F. do Amaral, A.J. Rosendale, R.E. Lee, Cryoprotectants and extreme freeze tolerance in a subarctic population of the wood frog., PLoS One. 10 (2015) e0117234. https://doi.org/10.1371/journal.pone.0117234.

[18] K.B. Storey, J.M. Storey, Biochemical adaptation to extreme environments, in: Integr. Physiol. Proteomics Post-Genomics Age, 2005: pp. 169-200. https://doi.org/10.1385/1-59259-925-7:169.

[19] K.B. Storey, J.M. Storey, Natural freeze survival in animals, Annu. Rev. Ecol. Syst. 27 (1996) 365-386. https://doi.org/10.1146/annurev.ecolsys.27.1.365.

[20] P.W. Hochachka, L.T. Buck, C.J. Doll, S.C. Land, Unifying theory of hypoxia tolerance: Molecular/metabolic defense and rescue mechanisms for surviving oxygen lack., Proc. Natl. Acad. Sci. U. S. A. 93 (1996) 9493-9498. https://doi.org/10.1073/pnas.93.18.9493.

[21] L.J. Hawkins, M. Wang, B. Zhang, Q. Xiao, H. Wang, K.B. Storey, Glucose and urea metabolic enzymes are differentially phosphorylated during freezing, anoxia, and dehydration exposures in a freeze tolerant frog, Comp. Biochem. Physiol. Part D Genomics Proteomics. 30 (2019) 1-13. https://doi.org/10.1016/j.cbd.2019.01.009.

[22] K.B. Storey, J.M. Storey, Molecular physiology of freeze tolerance in vertebrates, Physiol. Rev. 97 (2017) 623-665. https://doi.org/10.1152/physrev.00016.2016.

[23] V.E.M. Gerber, S. Wijenayake, K.B. Storey, Anti-apoptotic response during anoxia and recovery in a freeze-tolerant wood frog (Rana sylvatica)., PeerJ. 4 (2016) e1834. https://doi.org/10.7717/peerj.1834.

[24] R. Roufayel, K.K. Biggar, K.B. Storey, Regulation of cell cycle components during exposure to anoxia or dehydration stress in the wood frog, Rana sylvatica, J. Exp. Zool. Part A Ecol. Genet. Physiol. 315A (2011) 487-494. https://doi.org/10.1002/jez.696.

[25] C.-W. Wu, S.N. Tessier, K.B. Storey, Stress-induced antioxidant defense and protein chaperone response in the freeze-tolerant wood frog Rana sylvatica, Cell Stress Chaperones. 23 (2018) 1205-1217. https://doi.org/10.1007/s12192-018- 
0926-x.

[26] J.M. Storey, K.B. Storey, In defense of proteins: Chaperones respond to freezing, anoxia, or dehydration stress in tissues of freeze tolerant wood frogs, J. Exp. Zool. Part A Ecol. Integr. Physiol. 331 (2019) 392-402. https://doi.org/10.1002/jez.2306.

[27] K.B. Storey, Regulation of hypometabolism: Insights into epigenetic controls., J. Exp. Biol. 218 (2015) 150-9. https://doi.org/10.1242/jeb.106369.

[28] K.K. Biggar, K.B. Storey, The emerging roles of microRNAs in the molecular responses of metabolic rate depression, J. Mol. Cell Biol. 3 (2011) 167-175. https://doi.org/10.1093/jmcb/mjq045.

[29] K.B. Storey, J.M. Storey, Metabolic rate depression in animals: Transcriptional and translational controls, Biol. Rev. 79 (2004) 207-233. https://doi.org/10.1017/S1464793103006195.

[30] Z. Zhang, M. Guo, S. Zhao, W. Xu, J. Shao, F. Zhang, L. Wu, Y. Lu, S. Zheng, The update on transcriptional regulation of autophagy in normal and pathologic cells: A novel therapeutic target, Biomed. Pharmacother. 74 (2015) 17-29. https://doi.org/10.1016/j.biopha.2015.06.003.

[31] N. Mizushima, B. Levine, A.M. Cuervo, D.J. Klionsky, Autophagy fights disease through cellular self-digestion, Nature. 451 (2008) 1069-1075. https://doi.org/10.1038/nature06639.

[32] N. Mizushima, M. Komatsu, Autophagy: Renovation of cells and tissues, Cell. 147 (2011) 728-741. https://doi.org/10.1016/j.cell.2011.10.026.

[33] H.S. Jung, K.W. Chung, J. Won Kim, J. Kim, M. Komatsu, K. Tanaka, Y.H. Nguyen, T.M. Kang, K.H. Yoon, J.W. Kim, Y.T. Jeong, M.S. Han, M.K. Lee, K.W. Kim, J. Shin, M.S. Lee, Loss of autophagy diminishes pancreatic $\beta$ cell mass and function with resultant hyperglycemia, Cell Metab. 8 (2008) 318-324. https://doi.org/10.1016/j.cmet.2008.08.013.

[34] M. Koike, M. Shibata, M. Tadakoshi, K. Gotoh, M. Komatsu, S. Waguri, N. Kawahara, K. Kuida, S. Nagata, E. Kominami, K. Tanaka, Y. Uchiyama, Inhibition of autophagy prevents hippocampal pyramidal neuron death after hypoxic-ischemic injury, Am. J. Pathol. 172 (2008) 454-469. https://doi.org/10.2353/ajpath.2008.070876.

[35] K. Sun, X. Xie, Y. Liu, Z. Han, X. Zhao, N. Cai, S. Zhang, J. Song, L. Wei, Autophagy lessens ischemic liver injury by reducing oxidative damage, Cell Biosci. 3 (2013) 26. https://doi.org/10.1186/2045-3701-3-26.

[36] C. He, D.J. Klionsky, Regulation mechanisms and signaling pathways of autophagy, Annu. Rev. Genet. 43 (2009) 67-93. https://doi.org/10.1146/annurevgenet-102808-114910.

[37] J. Sakamaki, S. Wilkinson, M. Hahn, N. Tasdemir, J. O’Prey, W. Clark, A. Hedley, C. Nixon, J.S. Long, M. New, T. Van Acker, S.A. Tooze, S.W. Lowe, I. Dikic, K.M. Ryan, Bromodomain protein BRD4 is a transcriptional repressor of autophagy and lysosomal function, Mol. Cell. 66 (2017) 517-532.e9. https://doi.org/10.1016/j.molcel.2017.04.027. 
[38] J. Füllgrabe, M.A. Lynch-Day, N. Heldring, W. Li, R.B. Struijk, Q. Ma, O. Hermanson, M.G. Rosenfeld, D.J. Klionsky, B. Joseph, The histone H4 lysine 16 acetyltransferase hMOF regulates the outcome of autophagy, Nature. 500 (2013) 468-471. https://doi.org/10.1038/nature12313.

[39] C. Di Malta, L. Cinque, C. Settembre, Transcriptional regulation of autophagy: mechanisms and diseases., Front. Cell Dev. Biol. 7 (2019) 114. https://doi.org/10.3389/fcell.2019.00114.

[40] M. Sardiello, M. Palmieri, A. di Ronza, D.L. Medina, M. Valenza, V.A. Gennarino, C. Di Malta, F. Donaudy, V. Embrione, R.S. Polishchuk, S. Banfi, G. Parenti, E. Cattaneo, A. Ballabio, A Gene Network Regulating Lysosomal Biogenesis and Function, Science (80-. ). (2009). https://doi.org/10.1126/science.1174447.

[41] M. Palmieri, S. Impey, H. Kang, A. di Ronza, C. Pelz, M. Sardiello, A. Ballabio, Characterization of the CLEAR network reveals an integrated control of cellular clearance pathways, Hum. Mol. Genet. 20 (2011) 3852-3866. https://doi.org/10.1093/hmg/ddr306.

[42] C. Settembre, C. Di Malta, V.A. Polito, M.G. Arencibia, F. Vetrini, S. Erdin, S.U. Erdin, T. Huynh, D. Medina, P. Colella, M. Sardiello, D.C. Rubinsztein, A. Ballabio, TFEB links autophagy to lysosomal biogenesis, Science (80-. ). 332 (2011) 1429-1433. https://doi.org/10.1126/science.1204592.

[43] L.B. Frankel, A.H. Lund, MicroRNA regulation of autophagy, Carcinogenesis. 33 (2012) 2018-2025. https://doi.org/10.1093/carcin/bgs266.

[44] J. O’Brien, H. Hayder, Y. Zayed, C. Peng, Overview of microRNA biogenesis, mechanisms of actions, and circulation, Front. Endocrinol. (Lausanne). 9 (2018) 402. https://doi.org/10.3389/fendo.2018.00402.

[45] H. Hadj-Moussa, K.B. Storey, Micromanaging freeze tolerance: The biogenesis and regulation of neuroprotective microRNAs in frozen brains, Cell. Mol. Life Sci. 75 (2018) 3635-3647. https://doi.org/10.1007/s00018-018-2821-0.

[46] S.G. English, H. Hadj-Moussa, K.B. Storey, MicroRNAs regulate survival in oxygen-deprived environments, J. Exp. Biol. 221 (2018) jeb190579. https://doi.org/10.1242/jeb.190579.

[47] S. Bansal, B.E. Luu, K.B. Storey, MicroRNA regulation in heart and skeletal muscle over the freeze-thaw cycle in the freeze tolerant wood frog, J. Comp. Physiol. B. 186 (2016) 229-241. https://doi.org/10.1007/s00360-015-0951-3.

[48] H. Hadj-Moussa, J.A. Moggridge, B.E. Luu, J.F. Quintero-Galvis, J.D. GaitánEspitia, R.F. Nespolo, K.B. Storey, The hibernating South American marsupial, Dromiciops gliroides, displays torpor-sensitive microRNA expression patterns, Sci. Rep. 6 (2016) 24627. https://doi.org/10.1038/srep24627.

[49] H. Li, D. Miao, Q. Zhu, J. Huang, G. Lu, W. Xu, MicroRNA-17-5p contributes to osteoarthritis progression by binding p62/SQSTM1., Exp. Ther. Med. 15 (2018) 1789-1794. https://doi.org/10.3892/etm.2017.5622.

[50] A. Meenhuis, P.A. van Veelen, H. de Looper, N. van Boxtel, I.J. van den Berge, 
S.M. Sun, E. Taskesen, P. Stern, A.H. de Ru, A.J. van Adrichem, J. Demmers, M. Jongen-Lavrencic, B. Löwenberg, I.P. Touw, P.A. Sharp, S.J. Erkeland, MiR17/20/93/106 promote hematopoietic cell expansion by targeting sequestosome 1regulated pathways in mice, Blood. 118 (2011) 916-925. https://doi.org/10.1182/blood-2011-02-336487.

[51] Y. Xie, R. Kang, X. Sun, M. Zhong, J. Huang, D.J. Klionsky, D. Tang, Posttranslational modification of autophagy-related proteins in macroautophagy, Autophagy. 11 (2015) 28-45. https://doi.org/10.4161/15548627.2014.984267.

[52] Y.L. Deribe, T. Pawson, I. Dikic, Post-translational modifications in signal integration, Nat. Struct. Mol. Biol. 17 (2010) 666-672. https://doi.org/10.1038/nsmb.1842.

[53] D.G. McEwan, I. Dikic, The three musketeers of autophagy: Phosphorylation, ubiquitylation and acetylation, Trends Cell Biol. 21 (2011) 195-201. https://doi.org/10.1016/j.tcb.2010.12.006.

[54] T. Hanada, N.N. Noda, Y. Satomi, Y. Ichimura, Y. Fujioka, T. Takao, F. Inagaki, Y. Ohsumi, The Atg12-Atg5 conjugate has a novel E3-like activity for protein lipidation in autophagy, J. Biol. Chem. 282 (2007) 37298-37302. https://doi.org/10.1074/jbc.C700195200.

[55] T.A. Churchill, K.B. Storey, Metabolic responses to dehydration by liver of the wood frog, Rana sylvatica, Can. J. Zool. 72 (1994) 1420-1425. https://doi.org/10.1139/z94-188.

[56] R. Al-Attar, K.B. Storey, Effects of anoxic exposure on the nuclear factor of activated T cell (NFAT) transcription factors in the stress-tolerant wood frog., Cell Biochem. Funct. 36 (2018) 420-430. https://doi.org/10.1002/cbf.3362.

[57] S.A. Bustin, V. Benes, J.A. Garson, J. Hellemans, J. Huggett, M. Kubista, R. Mueller, T. Nolan, M.W. Pfaffl, G.L. Shipley, J. Vandesompele, C.T. Wittwer, The MIQE guidelines: Minimum information for publication of quantitative realtime PCR experiments, Clin. Chem. 55 (2009) 611-622. https://doi.org/10.1373/clinchem.2008.112797.

[58] W. Rennie, S. Kanoria, C. Liu, B. Mallick, D. Long, A. Wolenc, C.S. Carmack, J. $\mathrm{Lu}$, Y. Ding, STarMirDB: A database of microRNA binding sites, RNA Biol. 13 (2016) 554-560. https://doi.org/10.1080/15476286.2016.1182279.

[59] J. Zhang, K.B. Storey, Akt signaling and freezing survival in the wood frog, Rana sylvatica, Biochim. Biophys. Acta. 1830 (2013) 4828-37. https://doi.org/10.1016/j.bbagen.2013.06.020.

[60] S.L. Eaton, S.L. Roche, M. Llavero Hurtado, K.J. Oldknow, C. Farquharson, T.H. Gillingwater, T.M. Wishart, Total protein analysis as a reliable loading control for quantitative fluorescent western blotting, PLoS One. 8 (2013) e 72457. https://doi.org/10.1371/journal.pone.0072457.

[61] J. Zhang, K.B. Storey, RBioplot: An easy-to-use R pipeline for automated statistical analysis and data visualization in molecular biology and biochemistry, PeerJ. 4 (2016) e2436. https://doi.org/10.7717/peerj.2436. 
[62] G. Tettamanti, E. Carata, A. Montali, L. Dini, G.M. Fimia, Autophagy in development and regeneration: role in tissue remodelling and cell survival, Eur. Zool. J. 86 (2019) 113-131. https://doi.org/10.1080/24750263.2019.1601271.

[63] B. Levine, G. Kroemer, Autophagy in the pathogenesis of disease, Cell. 132 (2008) 27-42. https://doi.org/10.1016/j.cell.2007.12.018.

[64] W.A. Vistro, Y. Zhang, X. Bai, P. Yang, Y. Huang, W. Qu, A.S. Baloch, R. Wu, I. Tarique, Q. Chen, In vivo autophagy up-regulation of small intestine enterocytes in Chinese soft-shelled turtles during hibernation, Biomolecules. 9 (2019) 682. https://doi.org/10.3390/biom9110682.

[65] H. Chang, X. Peng, X. Yan, J. Zhang, S. Xu, H. Wang, Z. Wang, X. Ma, Y. Gao, Autophagy and Akt-mTOR signaling display periodic oscillations during torporarousal cycles in oxidative skeletal muscle of Daurian ground squirrels (Spermophilus dauricus), J. Comp. Physiol. B. 190 (2020) 113-123. https://doi.org/10.1007/s00360-019-01245-5.

[66] L. Shang, S. Chen, F. Du, S. Li, L. Zhao, X. Wang, Nutrient starvation elicits an acute autophagic response mediated by Ulk1 dephosphorylation and its subsequent dissociation from AMPK, Proc. Natl. Acad. Sci. 108 (2011) 4788-4793. https://doi.org/10.1073/pnas.1100844108.

[67] W. Li, Y. Yang, Z. Ba, S. Li, H. Chen, X. Hou, L. Ma, P. He, L. Jiang, L. Li, R. He, L. Zhang, D. Feng, MicroRNA-93 regulates hypoxia-induced autophagy by targeting ULK1, Oxid. Med. Cell. Longev. 2017 (2017) 1-13. https://doi.org/10.1155/2017/2709053.

[68] W. Tian, W. Li, Y. Chen, Z. Yan, X. Huang, H. Zhuang, W. Zhong, Y. Chen, W. Wu, C. Lin, H. Chen, X. Hou, L. Zhang, S. Sui, B. Zhao, Z. Hu, L. Li, D. Feng, Phosphorylation of ULK1 by AMPK regulates translocation of ULK1 to mitochondria and mitophagy, FEBS Lett. 589 (2015) 1847-1854. https://doi.org/10.1016/j.febslet.2015.05.020.

[69] D. Egan, J. Kim, R.J. Shaw, K.-L. Guan, The autophagy initiating kinase ULK1 is regulated via opposing phosphorylation by AMPK and mTOR., Autophagy. 7 (2011) 643-4. https://doi.org/10.4161/auto.7.6.15123.

[70] M.H. Rider, N. Hussain, S. Horman, S.M. Dilworth, K.B. Storey, Stress-induced activation of the AMP-activated protein kinase in the freeze-tolerant frog Rana sylvatica, Cryobiology. 53 (2006) 297-309.

https://doi.org/10.1016/j.cryobiol.2006.08.001.

[71] I.G. Ganley, D.H. Lam, J. Wang, X. Ding, S. Chen, X. Jiang, ULK1·ATG13·FIP200 complex mediates mTOR signaling and is essential for autophagy, J. Biol. Chem. 284 (2009) 12297-12305. https://doi.org/10.1074/jbc.M900573200.

[72] R. Al-attar, C.L. Childers, V.C. Nguyen, M.E. Pamenter, K.B. Storey, Differential protein phosphorylation is responsible for hypoxia-induced regulation of the $\mathrm{Akt} / \mathrm{mTOR}$ pathway in naked mole rats, Comp. Biochem. Physiol. Part A Mol. Integr. Physiol. 242 (2020) 110653. https://doi.org/10.1016/j.cbpa.2020.110653. 
[73] S.N. Tessier, J. Zhang, K.K. Biggar, C.W. Wu, F. Pifferi, M. Perret, K.B. Storey, Regulation of the PI3K/AKT pathway and fuel utilization during primate torpor in the gray mouse lemur, Microcebus murinus, Genomics, Proteomics Bioinforma. 13 (2015) 91-102. https://doi.org/10.1016/j.gpb.2015.03.006.

[74] B.E. Luu, S. Wijenayake, J. Zhang, S.N. Tessier, J.F. Quintero-Galvis, J.D. Gaitán-Espitia, R.F. Nespolo, K.B. Storey, Strategies of biochemical adaptation for hibernation in a South American marsupial, Dromiciops gliroides : 2. Control of the Akt pathway and protein translation machinery, Comp. Biochem. Physiol. Part B Biochem. Mol. Biol. 224 (2018) 19-25. https://doi.org/10.1016/j.cbpb.2017.12.006.

[75] N. Tamura, S. Kageyama, M. Komatsu, S. Waguri, Hyperosmotic stress induces unconventional autophagy independent of the Ulk1 complex, Mol. Cell. Biol. 39 (2019). https://doi.org/10.1128/MCB.00024-19.

[76] R.C. Russell, Y. Tian, H. Yuan, H.W. Park, Y.-Y. Chang, J. Kim, H. Kim, T.P. Neufeld, A. Dillin, K.-L. Guan, ULK1 induces autophagy by phosphorylating Beclin-1 and activating VPS34 lipid kinase, Nat. Cell Biol. 15 (2013) 741-750. https://doi.org/10.1038/ncb2757.

[77] S.F. Funderburk, Q.J. Wang, Z. Yue, The beclin 1-VPS34 complex - at the crossroads of autophagy and beyond, Trends Cell Biol. 20 (2010) 355-362. https://doi.org/10.1016/j.tcb.2010.03.002.

[78] K. Obara, Y. Ohsumi, Atg14: A key player in orchestrating autophagy, Int. J. Cell Biol. 2011 (2011) 1-7. https://doi.org/10.1155/2011/713435.

[79] K. Matsunaga, E. Morita, T. Saitoh, S. Akira, N.T. Ktistakis, T. Izumi, T. Noda, T. Yoshimori, Autophagy requires endoplasmic reticulum targeting of the PI3-kinase complex via Atg14L, J. Cell Biol. 190 (2010) 511-521. https://doi.org/10.1083/jcb.200911141.

[80] W. Fan, A. Nassiri, Q. Zhong, Autophagosome targeting and membrane curvature sensing by Barkor/Atg14(L), Proc. Natl. Acad. Sci. 108 (2011) 7769-7774. https://doi.org/10.1073/pnas.1016472108.

[81] A. Oberstein, P.D. Jeffrey, Y. Shi, Crystal structure of the Bcl-X L-beclin1 peptide complex, J. Biol. Chem. 282 (2007) 13123-13132. https://doi.org/10.1074/jbc.M700492200.

[82] X. Li, L. He, K.H. Che, S.F. Funderburk, L. Pan, N. Pan, M. Zhang, Z. Yue, Y. Zhao, Imperfect interface of Beclin1 coiled-coil domain regulates homodimer and heterodimer formation with Atg14L and UVRAG, Nat. Commun. 3 (2012) 662. https://doi.org/10.1038/ncomms 1648 .

[83] W. Huang, W. Choi, W. Hu, N. Mi, Q. Guo, M. Ma, M. Liu, Y. Tian, P. Lu, F.-L. Wang, H. Deng, L. Liu, N. Gao, L. Yu, Y. Shi, Crystal structure and biochemical analyses reveal beclin 1 as a novel membrane binding protein, Cell Res. 22 (2012) 473-489. https://doi.org/10.1038/cr.2012.24.

[84] R. Kang, H.J. Zeh, M.T. Lotze, D. Tang, The Beclin 1 network regulates autophagy and apoptosis, Cell Death Differ. 18 (2011) 571-580. 
https://doi.org/10.1038/cdd.2010.191.

[85] M.C. Maiuri, G. Le Toumelin, A. Criollo, J.-C. Rain, F. Gautier, P. Juin, E.

Tasdemir, G. Pierron, K. Troulinaki, N. Tavernarakis, J.A. Hickman, O. Geneste, G. Kroemer, Functional and physical interaction between Bcl-XL and a BH3-like domain in Beclin-1, EMBO J. 26 (2007) 2527-2539. https://doi.org/10.1038/sj.emboj.7601689.

[86] S. Pattingre, A. Tassa, X. Qu, R. Garuti, H.L. Xiao, N. Mizushima, M. Packer, M.D. Schneider, B. Levine, Bcl-2 antiapoptotic proteins inhibit Beclin 1dependent autophagy, Cell. 122 (2005) 927-939. https://doi.org/10.1016/j.cell.2005.07.002.

[87] Y. Wei, S. Pattingre, S. Sinha, M. Bassik, B. Levine, JNK1-mediated phosphorylation of $\mathrm{Bcl}-2$ regulates starvation-induced autophagy, Mol. Cell. 30 (2008) 678-688. https://doi.org/10.1016/j.molcel.2008.06.001.

[88] J.-M. Park, M. Seo, C.H. Jung, D. Grunwald, M. Stone, N.M. Otto, E. Toso, Y. Ahn, M. Kyba, T.J. Griffin, L. Higgins, D.-H. Kim, ULK1 phosphorylates Ser30 of BECN1 in association with ATG14 to stimulate autophagy induction, Autophagy. 14 (2018) 584-597. https://doi.org/10.1080/15548627.2017.1422851.

[89] J. Kim, Y.C. Kim, C. Fang, R.C. Russell, J.H. Kim, W. Fan, R. Liu, Q. Zhong, K.L. Guan, Differential regulation of distinct Vps34 complexes by AMPK in nutrient stress and autophagy, Cell. 152 (2013) 290-303. https://doi.org/10.1016/j.cell.2012.12.016.

[90] J. Evankovich, S.W. Cho, R. Zhang, J. Cardinal, R. Dhupar, L. Zhang, J.R. Klune, J. Zlotnicki, T. Billiar, A. Tsung, High mobility group box 1 release from hepatocytes during ischemia and reperfusion injury is mediated by decreased histone deacetylase activity., J. Biol. Chem. 285 (2010) 39888-97. https://doi.org/10.1074/jbc.M110.128348.

[91] D. Tang, R. Kang, K.M. Livesey, C.W. Cheh, A. Farkas, P. Loughran, G. Hoppe, M.E. Bianchi, K.J. Tracey, H.J. Zeh, M.T. Lotze, Endogenous HMGB1 regulates autophagy, J. Cell Biol. 190 (2010) 881-892. https://doi.org/10.1083/jcb.200911078.

[92] R. Kang, K.M. Livesey, H.J. Zeh, M.T. Lotze, D. Tang, HMGB1: A novel Beclin 1-binding protein active in autophagy, Autophagy. 6 (2010) 1209-1211. https://doi.org/10.4161/auto.6.8.13651.

[93] R. Liu, X. Zhi, Q. Zhong, ATG14 controls SNARE-mediated autophagosome fusion with a lysosome, Autophagy. 11 (2015) 847-849. https://doi.org/10.1080/15548627.2015.1037549.

[94] K.B. Storey, J.M. Storey, Metabolic rate depression: the biochemistry of mammalian hibernation., Adv. Clin. Chem. 52 (2010) 77-108. https://doi.org/10.1016/S0065-2423(10)52003-1.

[95] M.S. Wold, J. Lim, V. Lachance, Z. Deng, Z. Yue, ULK1-mediated phosphorylation of ATG14 promotes autophagy and is impaired in Huntington's disease models, Mol. Neurodegener. 11 (2016) 76. https://doi.org/10.1186/s13024- 
016-0141-0.

[96] N. Mizushima, A. Yamamoto, M. Hatano, Y. Kobayashi, Y. Kabeya, K. Suzuki, T. Tokuhisa, Y. Ohsumi, T. Yoshimori, Dissection of autophagosome formation using Apg5-deficient mouse embryonic stem cells, J. Cell Biol. 152 (2001) 657668. https://doi.org/10.1083/jcb.152.4.657.

[97] A. Kuma, N. Mizushima, N. Ishihara, Y. Ohsumi, Formation of the 350-kDa Apg12-Apg5·Apg16 multimeric complex, mediated by Apg16 oligomerization, is essential for autophagy in yeast, J. Biol. Chem. 277 (2002) 18619-18625. https://doi.org/10.1074/jbc.M111889200.

[98] N. Mizushima, Mouse Apg16L, a novel WD-repeat protein, targets to the autophagic isolation membrane with the Apg12-Apg5 conjugate, J. Cell Sci. 116 (2003) 1679-1688. https://doi.org/10.1242/jcs.00381.

[99] S. Yousefi, R. Perozzo, I. Schmid, A. Ziemiecki, T. Schaffner, L. Scapozza, T. Brunner, H.-U. Simon, Calpain-mediated cleavage of Atg5 switches autophagy to apoptosis, Nat. Cell Biol. 8 (2006) 1124-1132. https://doi.org/10.1038/ncb1482.

[100] I. Tanida, T. Ueno, E. Kominami, Human light chain 3/MAP1LC3B is cleaved at its carboxyl-terminal Met121 to expose Gly120 for lipidation and targeting to autophagosomal membranes., J. Biol. Chem. 279 (2004) 47704-10. https://doi.org/10.1074/jbc.M407016200.

[101] G. Runwal, E. Stamatakou, F.H. Siddiqi, C. Puri, Y. Zhu, D.C. Rubinsztein, LC3positive structures are prominent in autophagy-deficient cells, Sci. Rep. 9 (2019) 10147. https://doi.org/10.1038/s41598-019-46657-z.

[102] C. Otomo, Z. Metlagel, G. Takaesu, T. Otomo, Structure of the human ATG12 ATG5 conjugate required for LC3 lipidation in autophagy, Nat. Struct. Mol. Biol. 20 (2013) 59-66. https://doi.org/10.1038/nsmb.2431.

[103] M. Li, Y. Fu, Z. Yang, X.-M. Yin, Measurement of the activity of the Atg4 cysteine proteases, in: Methods Enzymol., 2017: pp. 207-225. https://doi.org/10.1016/bs.mie.2016.10.024.

[104] Y. Kabeya, LC3, a mammalian homologue of yeast Apg8p, is localized in autophagosome membranes after processing, EMBO J. 19 (2000) 5720-5728. https://doi.org/10.1093/emboj/19.21.5720.

[105] E. Shvets, E. Fass, R. Scherz-Shouval, Z. Elazar, The N-terminus and Phe52 residue of LC3 recruit p62/SQSTM1 into autophagosomes, J. Cell Sci. 121 (2008) 2685-2695. https://doi.org/10.1242/jcs.026005.

[106] G. Bjørkøy, T. Lamark, A. Brech, H. Outzen, M. Perander, A. Øvervatn, H. Stenmark, T. Johansen, p62/SQSTM1 forms protein aggregates degraded by autophagy and has a protective effect on huntingtin-induced cell death, J. Cell Biol. 171 (2005) 603-614. https://doi.org/10.1083/jcb.200507002.

[107] J. Diao, R. Liu, Y. Rong, M. Zhao, J. Zhang, Y. Lai, Q. Zhou, L.M. Wilz, J. Li, S. Vivona, R.A. Pfuetzner, A.T. Brunger, Q. Zhong, ATG14 promotes membrane tethering and fusion of autophagosomes to endolysosomes, Nature. 520 (2015) 563-566. https://doi.org/10.1038/nature14147. 
[108] Z. Zhang, R. Singh, M. Aschner, Methods for the detection of autophagy in mammalian cells, Curr. Protoc. Toxicol. 69 (2016).

https://doi.org/10.1002/cptx.11.

[109] N. Mizushima, T. Yoshimori, B. Levine, Methods in mammalian autophagy research, Cell. 140 (2010) 313-326. https://doi.org/10.1016/j.cell.2010.01.028.

[110] D.J. Klionsky, The molecular machinery of autophagy: Unanswered questions, J. Cell Sci. 118 (2005) 7-18. https://doi.org/10.1242/jcs.01620.

[111] J. Füllgrabe, G. Ghislat, D.-H. Cho, D.C. Rubinsztein, Transcriptional regulation of mammalian autophagy at a glance, J. Cell Sci. 129 (2016) 3059-3066. https://doi.org/10.1242/jcs.188920.

[112] C. Settembre, R. Zoncu, D.L. Medina, F. Vetrini, S. Erdin, S. Erdin, T. Huynh, M. Ferron, G. Karsenty, M.C. Vellard, V. Facchinetti, D.M. Sabatini, A. Ballabio, A lysosome-to-nucleus signalling mechanism senses and regulates the lysosome via mTOR and TFEB, EMBO J. 31 (2012) 1095-1108. https://doi.org/10.1038/emboj.2012.32.

[113] D.L. Medina, S. Di Paola, I. Peluso, A. Armani, D. De Stefani, R. Venditti, S. Montefusco, A. Scotto-Rosato, C. Prezioso, A. Forrester, C. Settembre, W. Wang, Q. Gao, H. Xu, M. Sandri, R. Rizzuto, M.A. De Matteis, A. Ballabio, Lysosomal calcium signalling regulates autophagy through calcineurin and TFEB, Nat. Cell Biol. 17 (2015) 288-299. https://doi.org/10.1038/ncb3114.

[114] Z. Wang, N. Wang, P. Liu, Q. Chen, H. Situ, T. Xie, J. Zhang, C. Peng, Y. Lin, J. Chen, MicroRNA-25 regulates chemoresistance-associated autophagy in breast cancer cells, a process modulated by the natural autophagy inducer isoliquiritigenin, Oncotarget. 5 (2014). https://doi.org/10.18632/oncotarget.2192.

[115] D.Z. John Clotaire, B. Zhang, N. Wei, R. Gao, F. Zhao, Y. Wang, M. Lei, W. Huang, miR-26b inhibits autophagy by targeting ULK2 in prostate cancer cells, Biochem. Biophys. Res. Commun. 472 (2016) 194-200.

https://doi.org/10.1016/j.bbrc.2016.02.093.

[116] H. Zhu, H. Wu, X. Liu, B. Li, Y. Chen, X. Ren, C.-G. Liu, J.-M. Yang, Regulation of autophagy by a beclin 1-targeted microRNA, miR-30a, in cancer cells, Autophagy. 5 (2009) 816-823. https://doi.org/10.4161/auto.9064.

[117] Y. Yu, L. Cao, L. Yang, R. Kang, M. Lotze, D. Tang, microRNA 30A promotes autophagy in response to cancer therapy, Autophagy. 8 (2012) 853-855. https://doi.org/10.4161/auto.20053.

[118] X. Yang, X. Zhong, J.L. Tanyi, J. Shen, C. Xu, P. Gao, T.M. Zheng, A. DeMichele, L. Zhang, mir-30d Regulates multiple genes in the autophagy pathway and impairs autophagy process in human cancer cells, Biochem. Biophys. Res. Commun. 431 (2013) 617-622. https://doi.org/10.1016/j.bbrc.2012.12.083.

[119] Z. Zhai, F. Wu, A.Y. Chuang, J.H. Kwon, miR-106b fine tunes ATG16L1 expression and autophagic activity in intestinal epithelial HCT116 cells, Inflamm. Bowel Dis. 19 (2013) 2295-2301. https://doi.org/10.1097/MIB.0b013e31829e71cf. 
[120] H. Wu, F. Wang, S. Hu, C. Yin, X. Li, S. Zhao, J. Wang, X. Yan, MiR-20a and miR-106b negatively regulate autophagy induced by leucine deprivation via suppression of ULK1 expression in C2C12 myoblasts, Cell. Signal. 24 (2012) 2179-2186. https://doi.org/10.1016/j.cellsig.2012.07.001.

[121] K. Liu, J. Huang, M. Xie, Y. Yu, S. Zhu, R. Kang, L. Cao, D. Tang, X. Duan, MIR34A regulates autophagy and apoptosis by targeting HMGB1 in the retinoblastoma cell, Autophagy. 10 (2014) 442-452. https://doi.org/10.4161/auto.27418.

[122] P.J. Morin, K.B. Storey, Evidence for a reduced transcriptional state during hibernation in ground squirrels, Cryobiology. 53 (2006) 310-318. https://doi.org/10.1016/j.cryobiol.2006.08.002. 


\section{Tables}

Table 2.1: Nucleotide sequences of miRNA specific forward primers, universal primer and the stem-loop adapter.

\begin{tabular}{ll}
\hline Target & Primer Sequence (5'-3') \\
\hline miR-17-5p & ACACTCCAGCTGGGCAAAGTGCTTACAGT \\
miR-20-5p & ACACTCCAGCTGGGCAAAGTGCTCATAGT \\
miR-25-3p & ACACTCCAGCTGGGCATTGCACTTGTCTC \\
miR-26-5p & ACACTCCAGCTGGGTTCAAGTAATCCAGG \\
miR-30a-5p & ACACTCCAGCTGGGTGTAAACATCCTCGA \\
miR-30d-5p & ACACTCCAGCTGGGTGTAAACATCCCCGA \\
mir-34a-5p & ACACTCCAGCTGGGTGGCAGTGTCTTAGC \\
miR-93-5p & ACACTCCAGCTGGGCAAAGTGCTGTTCGTGC \\
mir-106b-5p & ACACTCCAGCTGGGTAAAGTGCTGACAGTGC \\
miR-124-5p & ACACTCCAGCTGGGCGTGTTCACAGCGGA \\
Snord-24-17bp & ACACTCCAGCTGGGTGCAGATGATGTAAAA \\
Snord-74a-15bp & ACACTCCAGCTGGGGTTGTCAGCTATCCA \\
5S RNA & GGCCTGGTTAGTACTTGGATG \\
Universal Primer & CTCACAGTACGTTGGTATCCTTGTG \\
Stem-loop Adapter & CTCACAGTACGTTGGTATCCTTGTGATGTTCGA \\
\hline
\end{tabular}

Table 2.2: Nucleotide sequences (5'-3') of forward and reverse primers used for mRNA analysis.

\begin{tabular}{lll} 
& Forward & Reverse \\
\cline { 2 - 3 } p62 & GGCCTTTCTCTGCTGAATGA & GGGCAGATGAGGCACTTAAA \\
$\beta-$ & AGAAGTCGTGCCAGGCATCA & AGGAGGAAGCTATCCGTGTT \\
actin & AGATATA
\end{tabular}




\section{Figures}

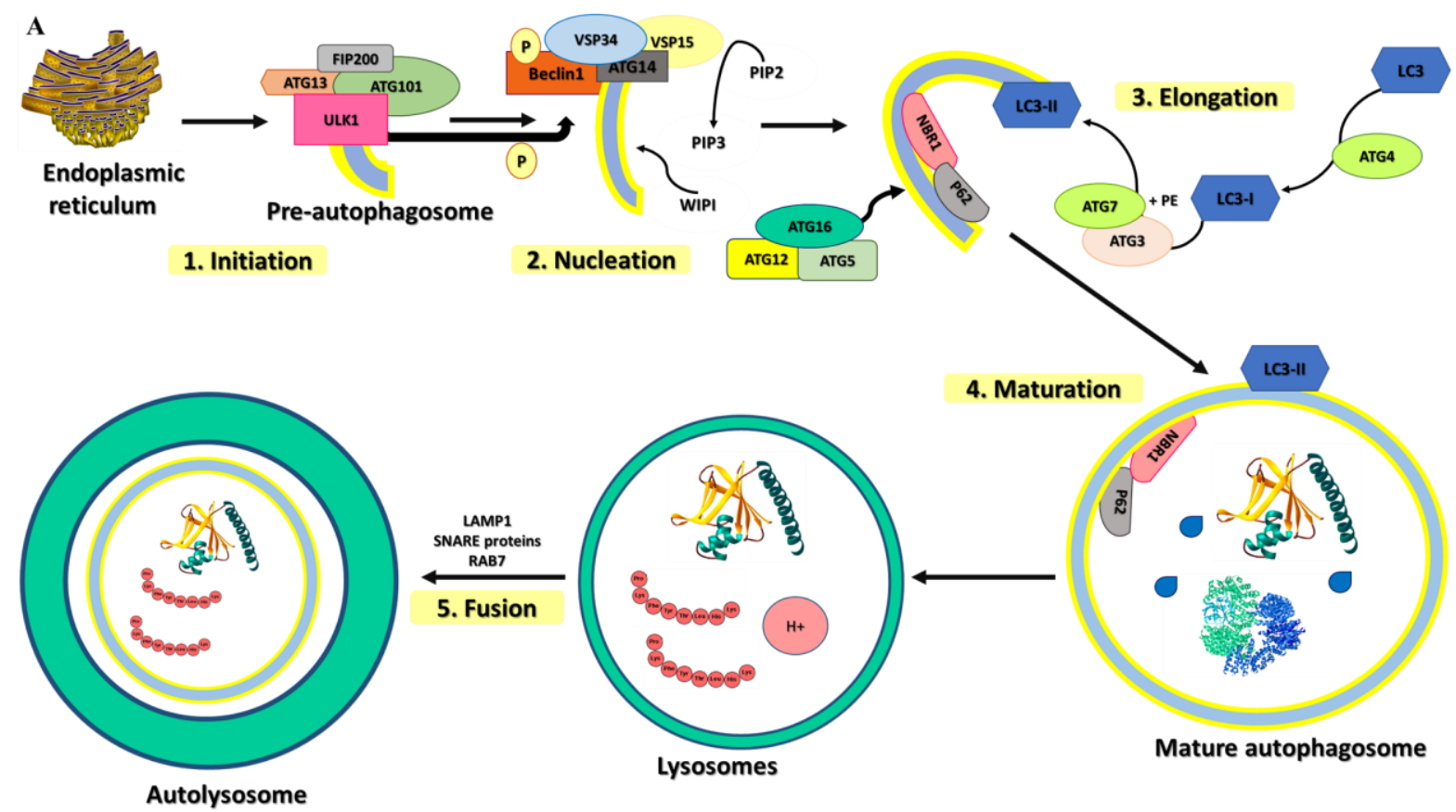

Figures 2.1: Schematic diagram showing different phases of autophagy. The initiation of autophagosome formation is regulated by many protein kinases including AMPK and mTORC1 that function oppositely to each other. These two kinases maintain cellular homeostasis by balancing anabolic and catabolic responses in the cell; where AMPK phosphorylation (S317, S467, S555, T575, S637, and S777) under ATP depleting conditions and mTORC1 phosphorylation (S638 and S758) under nutrient deprived conditions promote the activation and deactivation of ULK1, respectively. ULK1, a key initiator of autophagosome formation, is a serine/threonine kinase that forms a complex with ATG13, FIP200 and ATG101 [74-76]. Both ATG13 and FIP200 promote the stabilization of ULK1, promote its catalytic activity, and facilitate the translocation of the complex to the isolation membrane (pre-autophagosomal structure) before starting the omegasome formation. At the initiation site, the p-ULK1 complex promotes the recruitment of multiple other factors and complexes including the nucleation VPS34 complex, which consists of the class III phosphatidylinositol (PtdIns) kinase complex (VPS34, VPS15, beclin1 and ATG14. Beclin1 activation is mediated by phosphorylation, either by AMPK (S93 in humans) or by ULK1 at S30, and these events are necessary for induction of autophagy. The VPS34 complex promotes the production of PI3P at the growing phagophore, resulting in the recruitment of WIPI proteins, the maturation of the phagosome and the recruitment of the elongation complex. ATG12-ATG5-ATG16L (the elongation complex) functions as a ubiquitin-like conjugating complex that is necessary for phagophore elongation and LC3 recruitment to the growing phagophore. LC3 is then cleaved by ATG4 to LC3I which allows it to form a complex with the E1-like ATG7 and the E2-like ATG3 before getting conjugated with phosphatidylethanolamine (PE, LC3II). WIPI proteins also recruit cargo-recognition proteins such as p62 and NBR1 (neighbor of BRCA1 gene 1) which can bind to both LC3 on the maturing autophagosome and act as 
receptors for ubiquitinated targets for their selective degradation. Both p62 and NBR1 are degraded with the ubiquitinated cargo, thus serving as a marker to measure autophagic flux in cells. Once autophagosome formation and closure has been completed, they are delivered to the perinuclear region, where they can fuse with lysosomes to help many proteins including the those in the SNAREs family. 


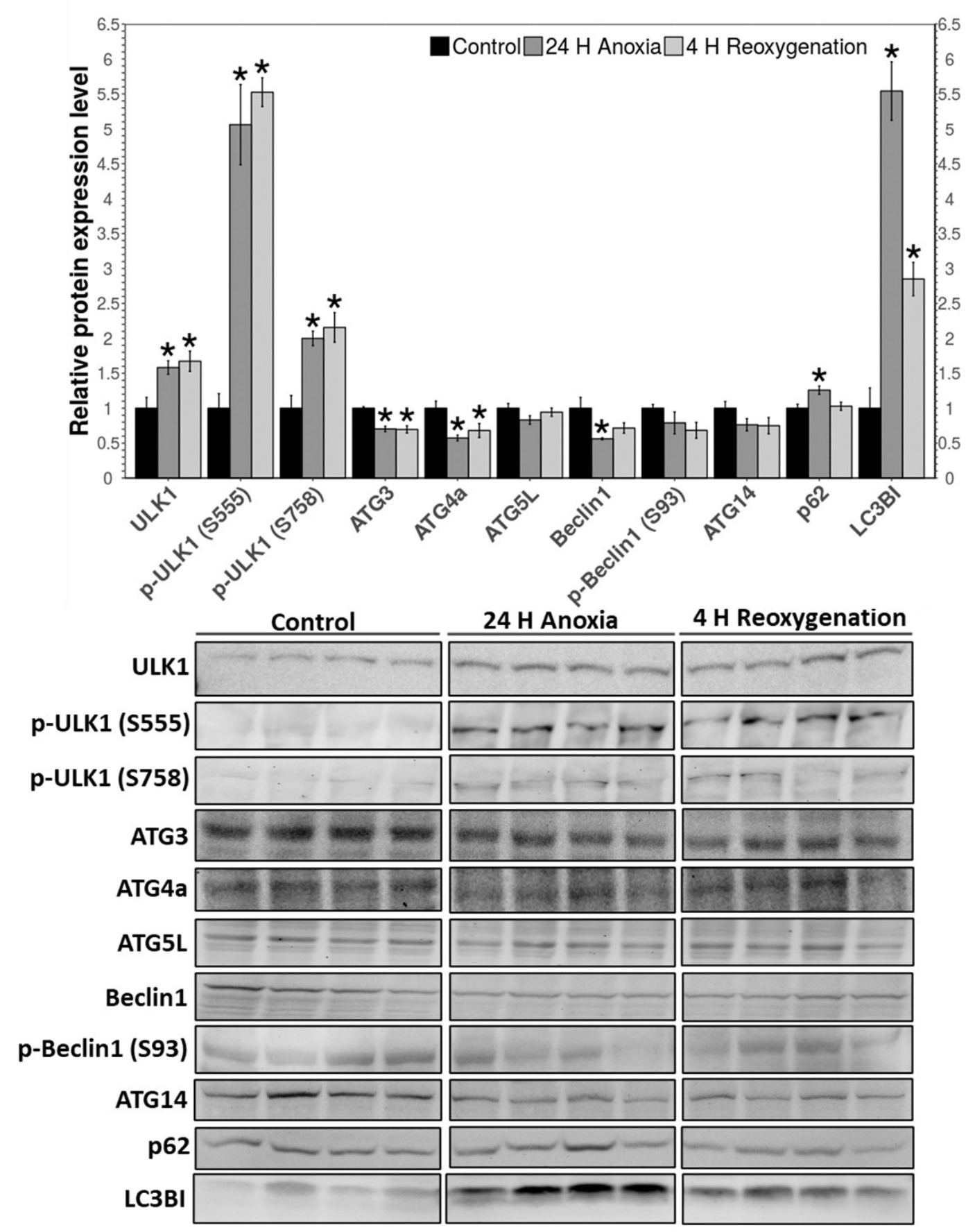

Figure 2.2: Relative protein levels of key proteins involved in autophagosome formation in liver over the anoxia/reoxygenation cycle measured by immunoblotting. Histogram shows means \pm SEM, $n=4$ independent biological replicates obtained from different animals. The corresponding enhanced chemiluminescence bands are also shown. Significance difference from the control $(\mathrm{p}<0.05)$ is denoted by an asterisk $(*)$ as determined using a one-way ANOVA with Dunnett's post-hoc test. 


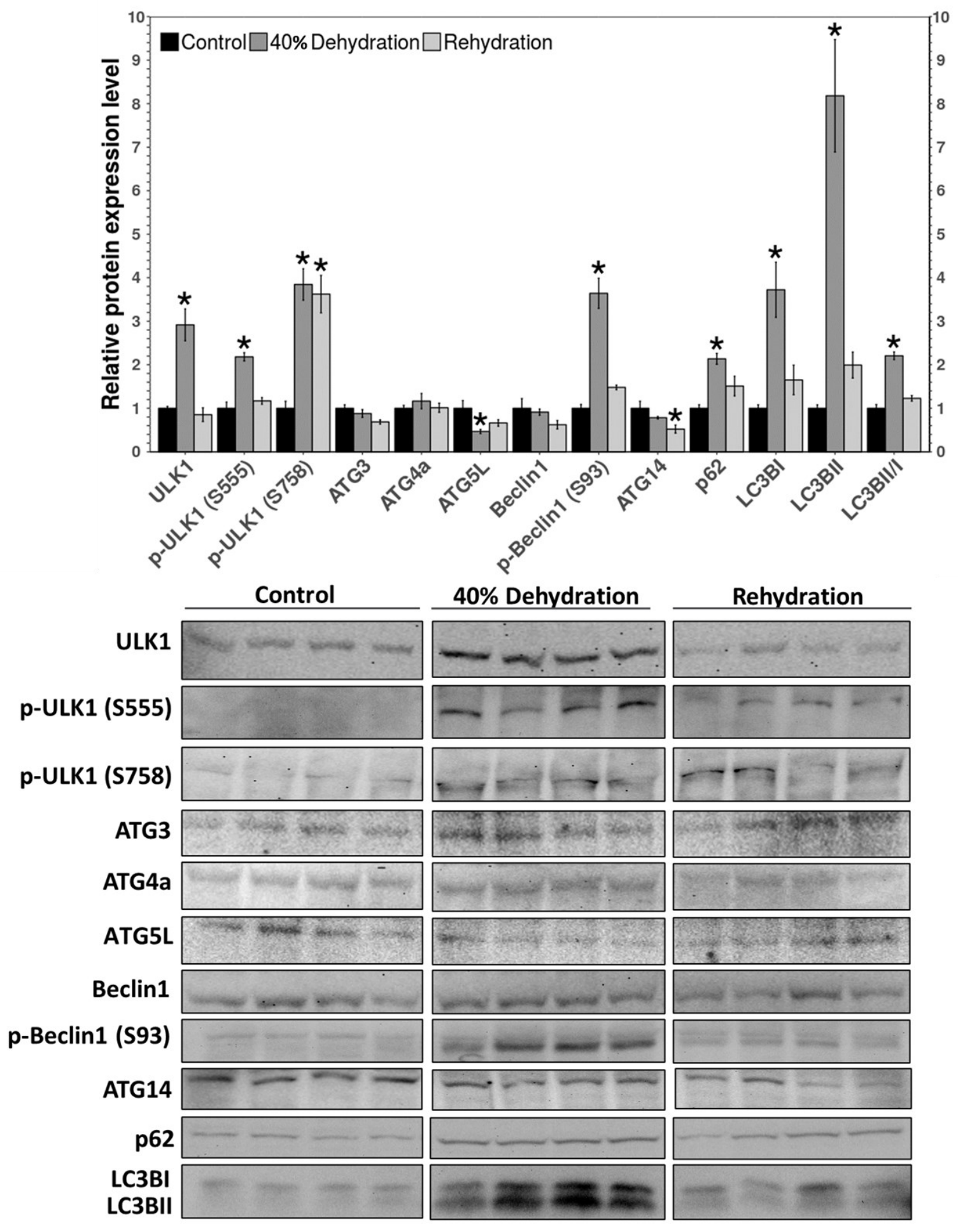

Figure 2.3: Relative protein expression levels of key proteins involved in autophagosome formation in liver over the dehydration/rehydration cycle measured using immunoblotting. For more information, see Figure 2.2. 


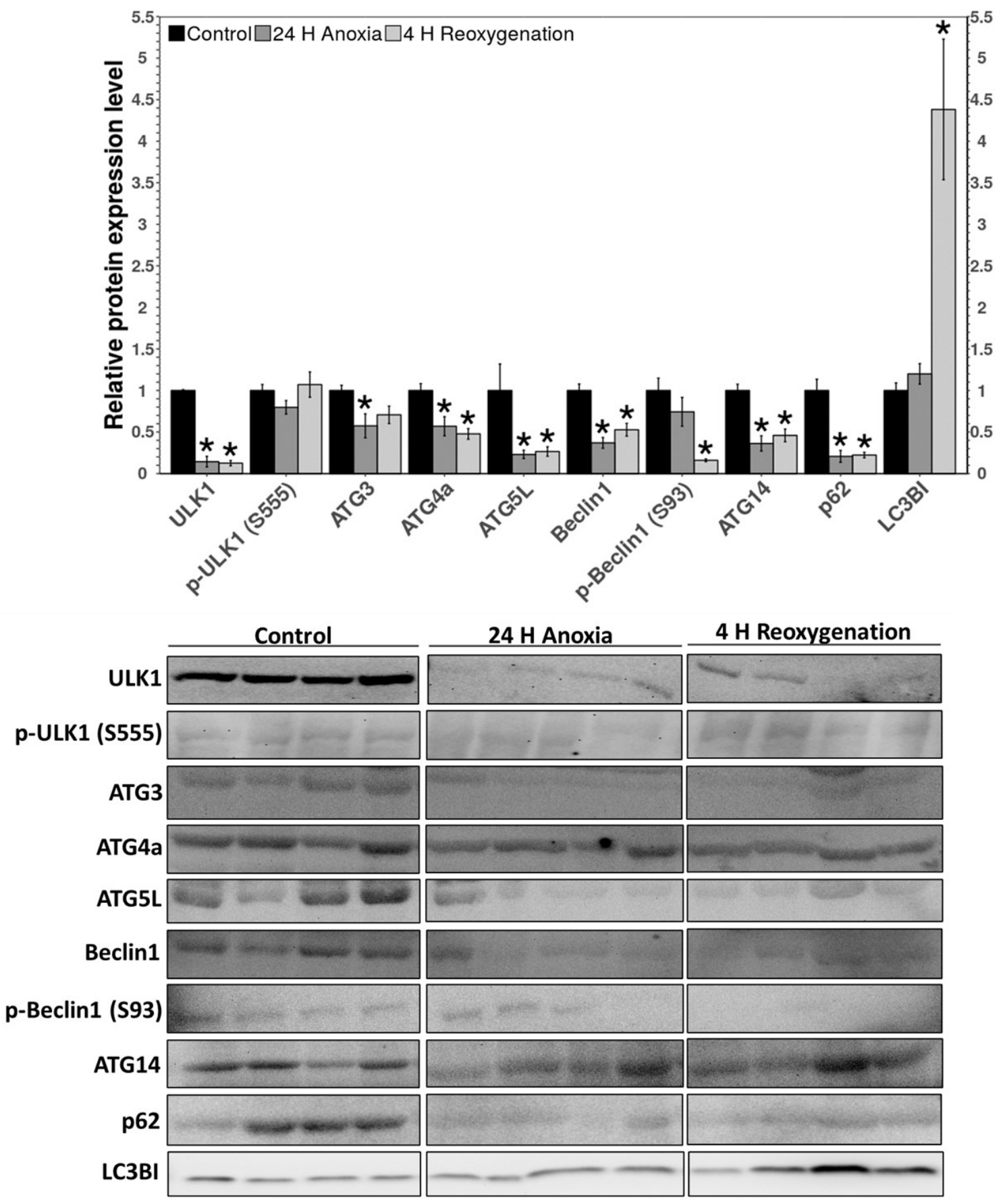

Figure 2.4: Relative protein expression levels of key proteins involved in autophagosome formation in skeletal muscle over the anoxia/reoxygenation cycle measured using immunoblotting. For more information, see Figure 2.2. 


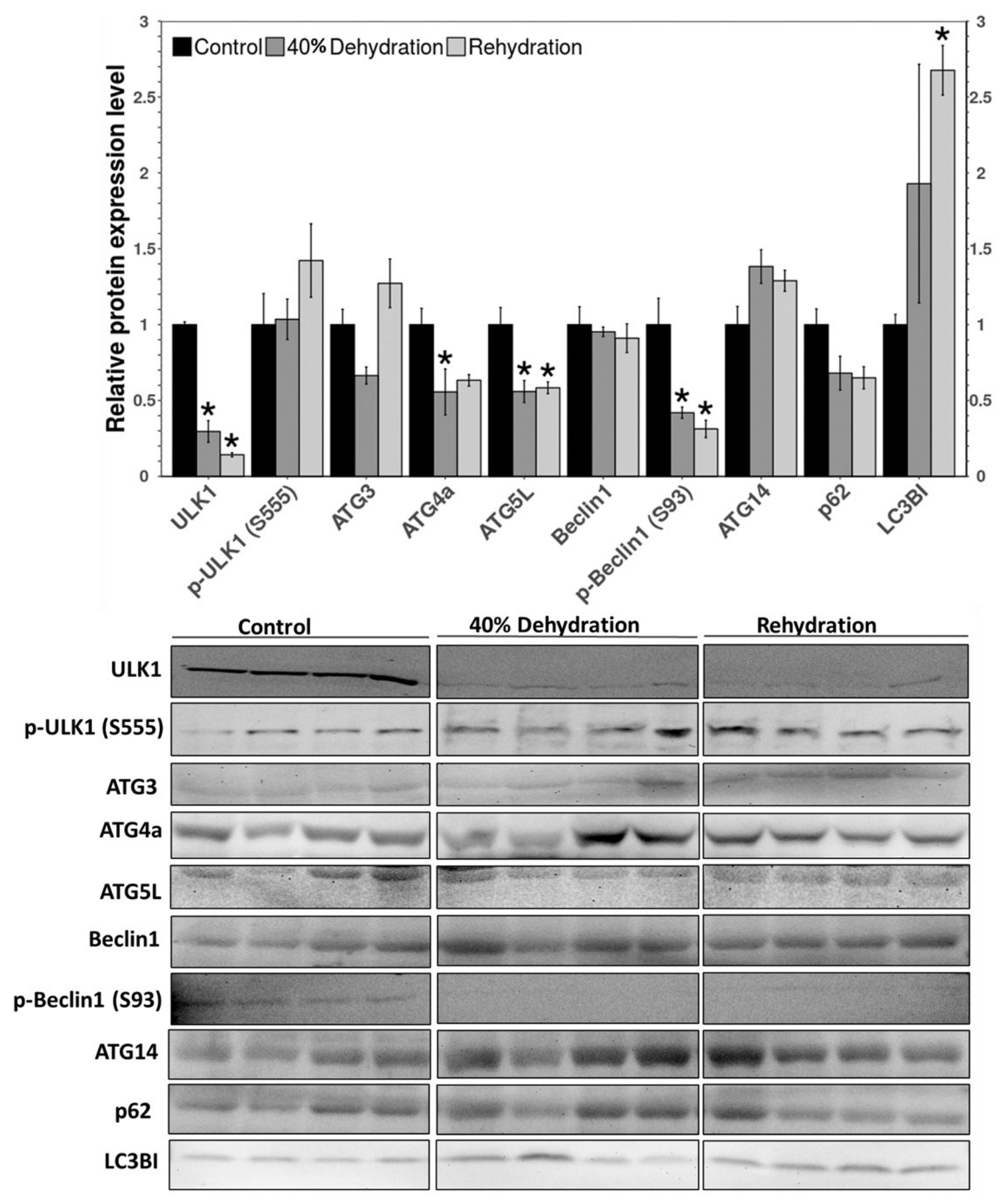

Figure 2.5: Relative protein expression levels of key proteins involved in autophagosome formation in skeletal muscle over the dehydration/rehydration cycle measured using immunoblotting. For more information, see Figure 2.2. 


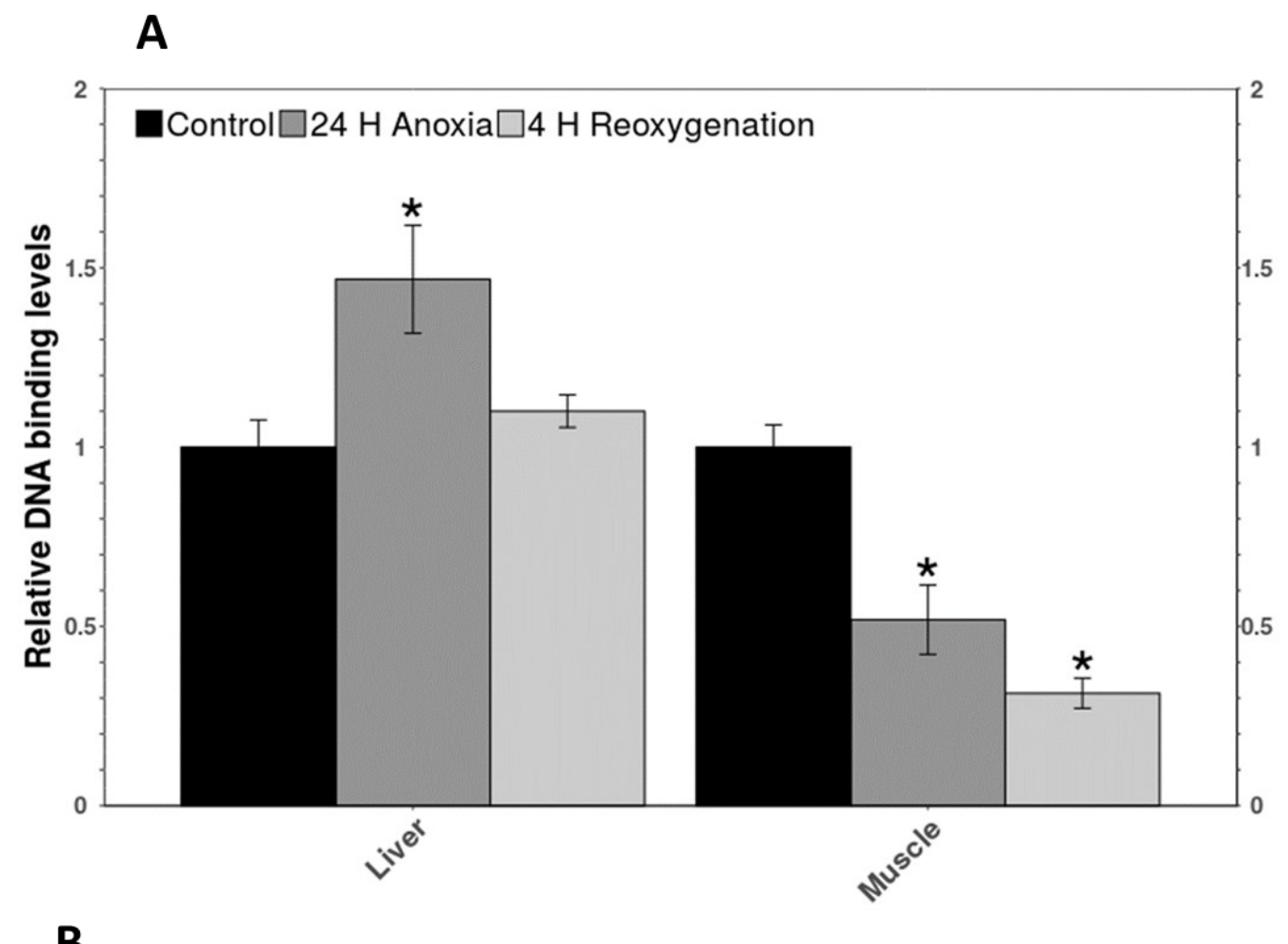

B

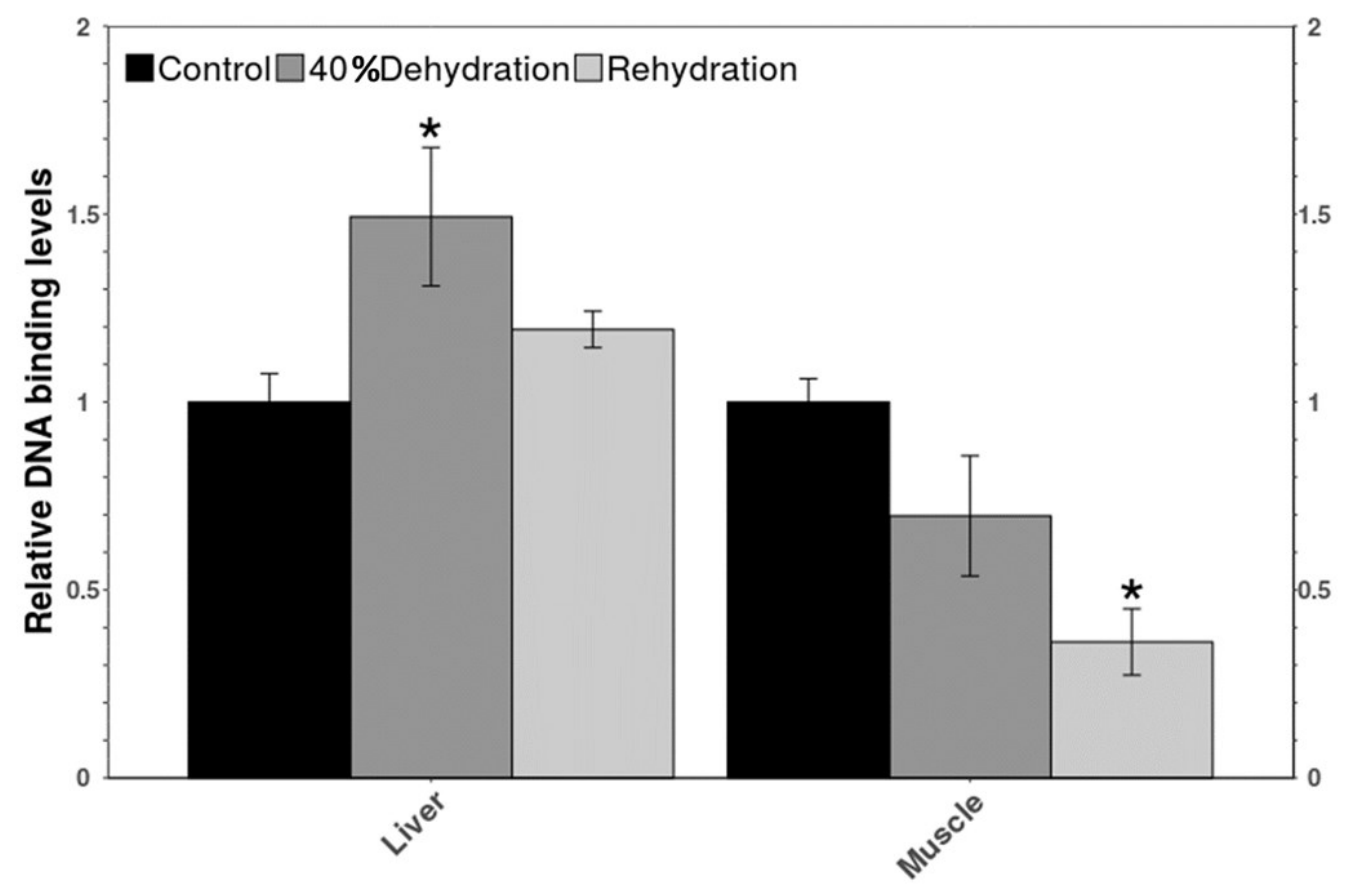

Figure 2.6: Relative DNA binding activity of TFEB to its consensus sequence in liver and muscle measured by DPI-ELISA. Measurements were done using tissues of wood frogs exposed to A) anoxia/reoxygenation, and B) dehydration/rehydration cycle. For more information, see Figure 2.2. 

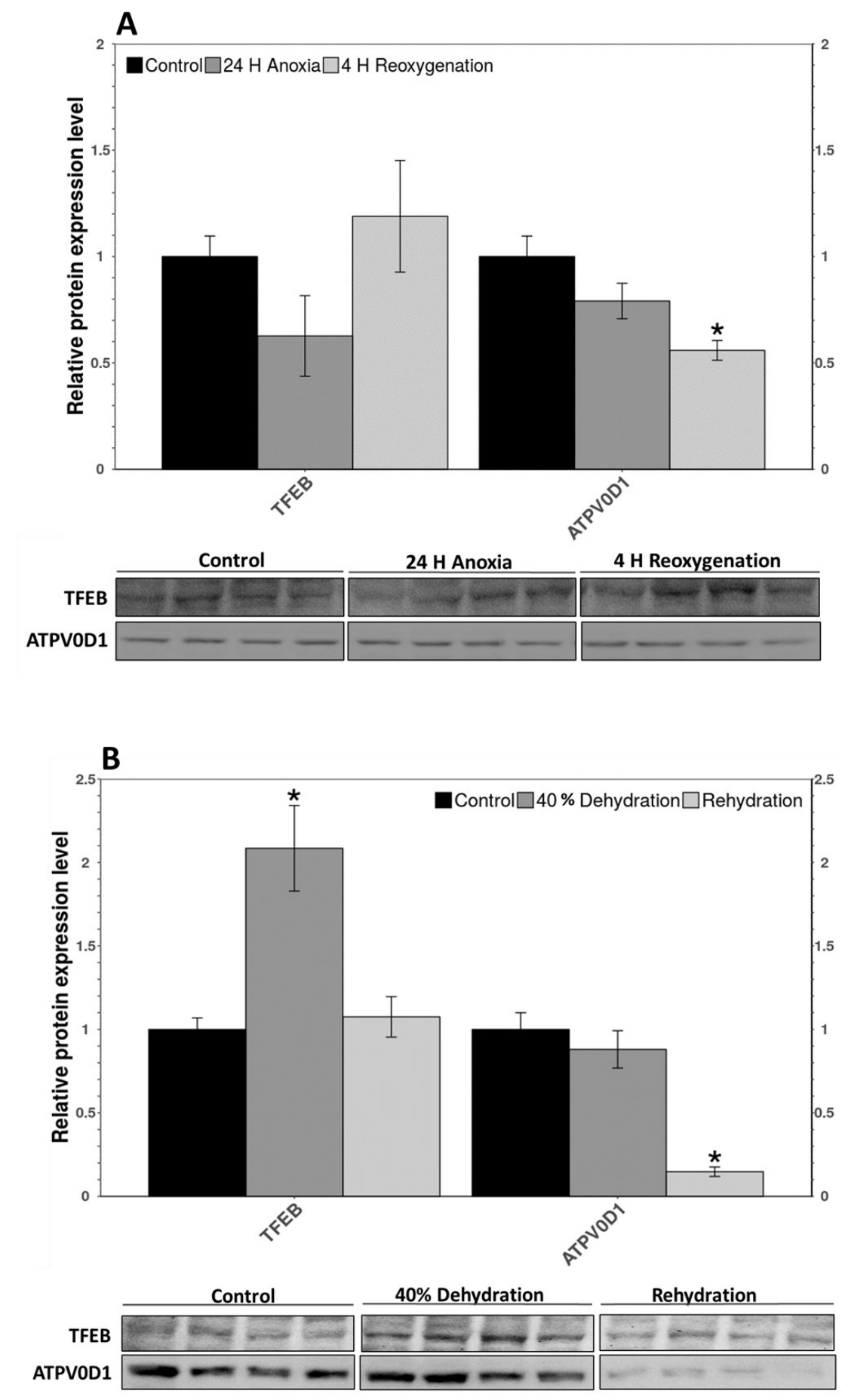

Figure 2.7: Relative protein expression levels of TFEB and one of its downstream targets, ATP6V0D1, in liver measured by immunoblotting. Measurements were done using tissues of wood frogs exposed to A) anoxia/reoxygenation, and B) dehydration/rehydration cycle. For more information, see Figure 2.2. 

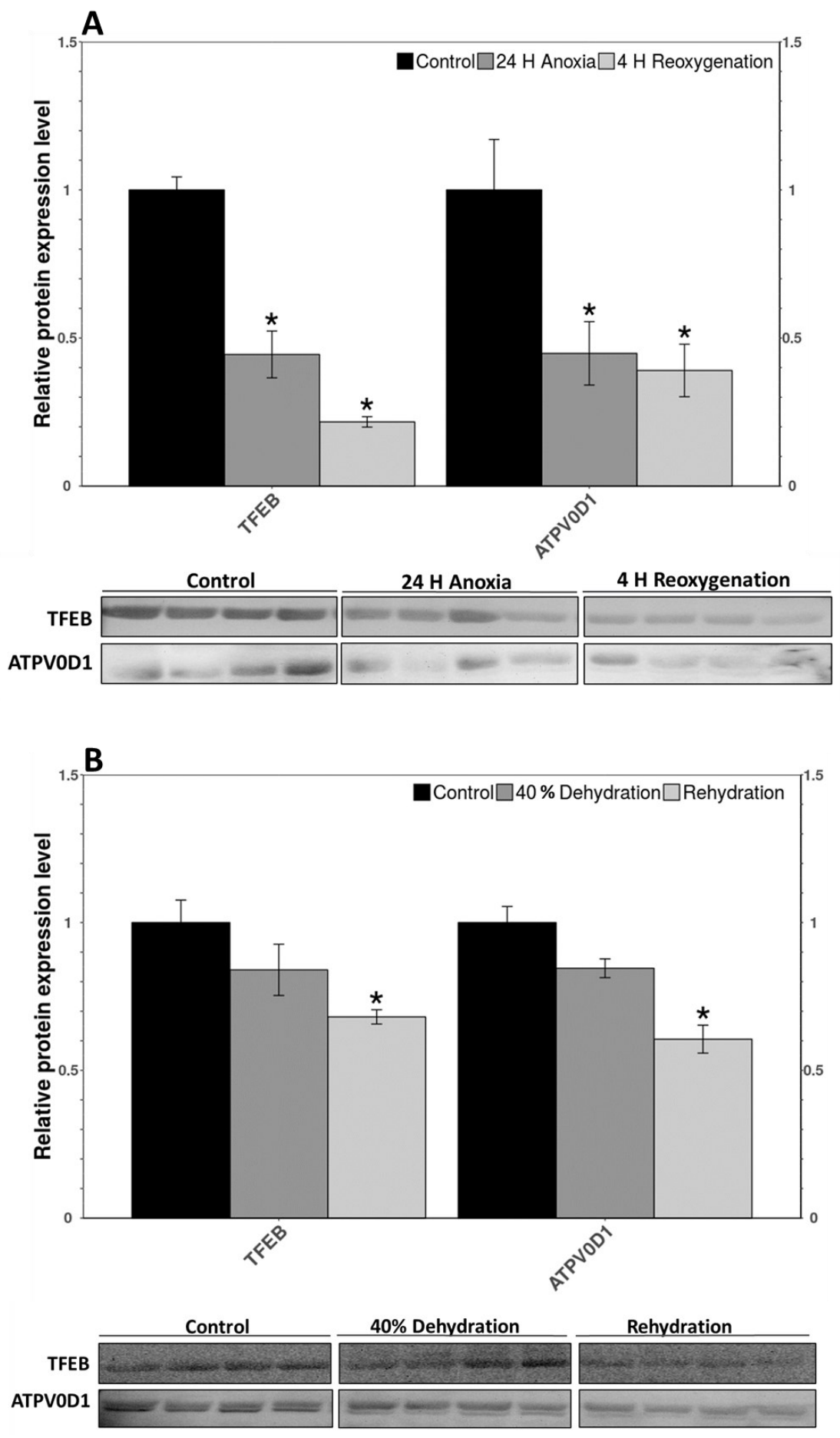

Figure 2.8: Relative protein expression levels of TFEB and one of its downstream targets, ATP6V0D1 in muscle measured by immunoblotting. Measurements were done using tissues of wood frogs exposed to A) anoxia/reoxygenation, and B) dehydration/rehydration cycle. For more information, see Figure 2.2. 

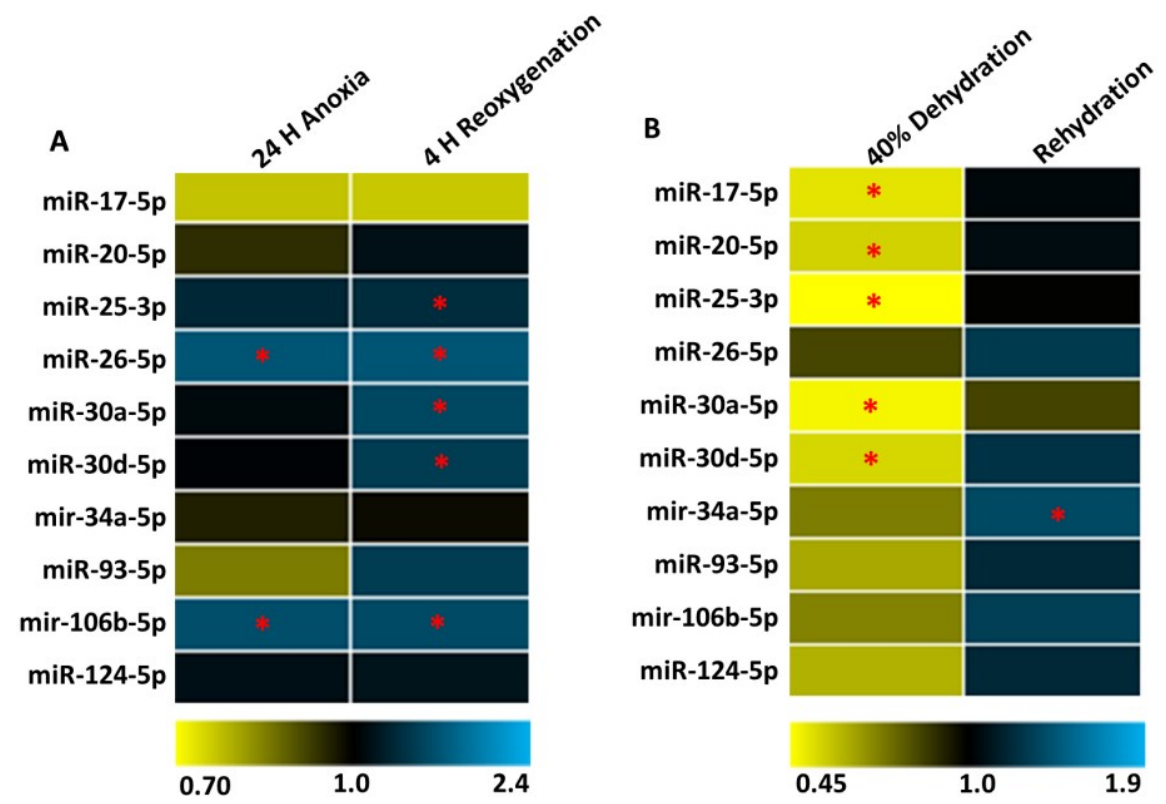

Figure 2.9: Heatmap of relative transcript levels of select autophagy-regulating miRNAs in liver measured using RT-qPCR. Data are presented as heat maps for A) anoxia/reoxygenation and B) dehydration/rehydration cycles. Significance difference from the control $(\mathrm{p}<0.05)$ is denoted by an asterisk $(*)$ as determined using a one-way ANOVA with Dunnett's post-hoc test. 

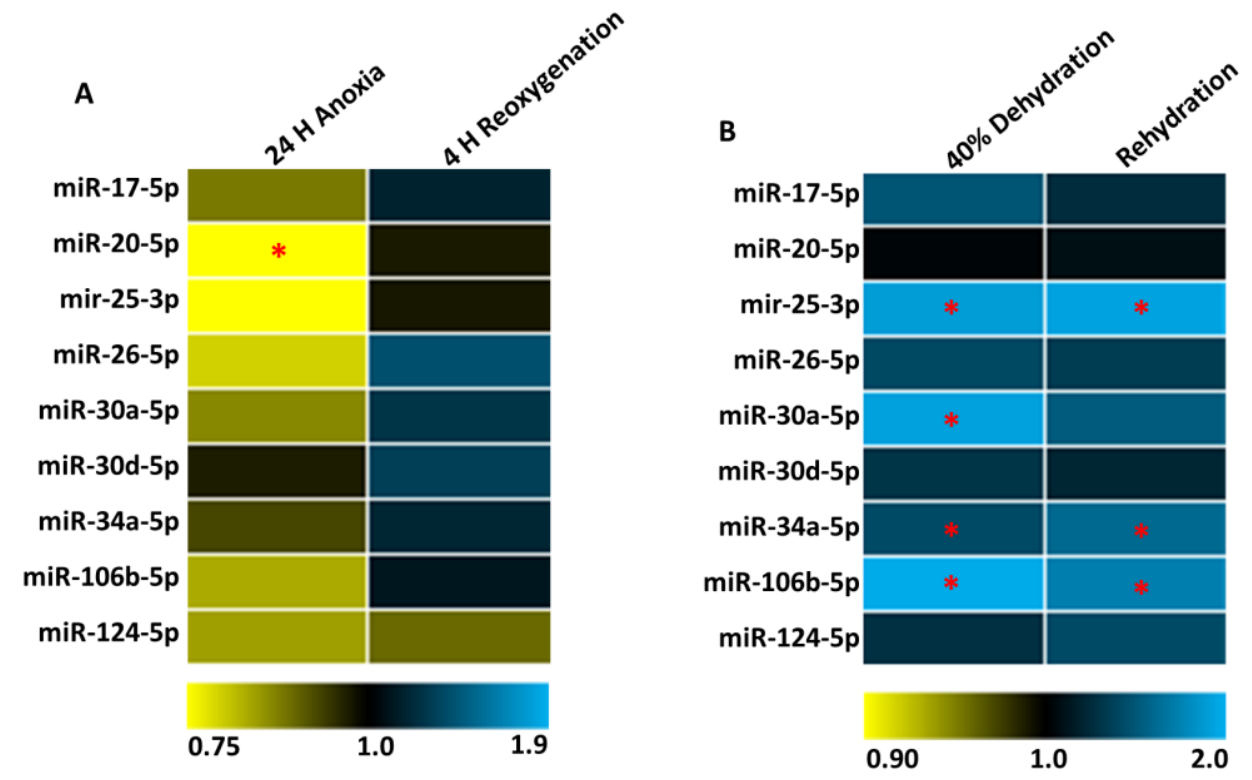

Figure 2.10: Heatmap of relative transcript levels of select autophagy-regulating miRNAs in skeletal muscle measured using RT-qPCR. Data are presented as heat maps for the A) anoxia/reoxygenation and B) dehydration/rehydration cycles. For more information, see Figure 2.9. 
A

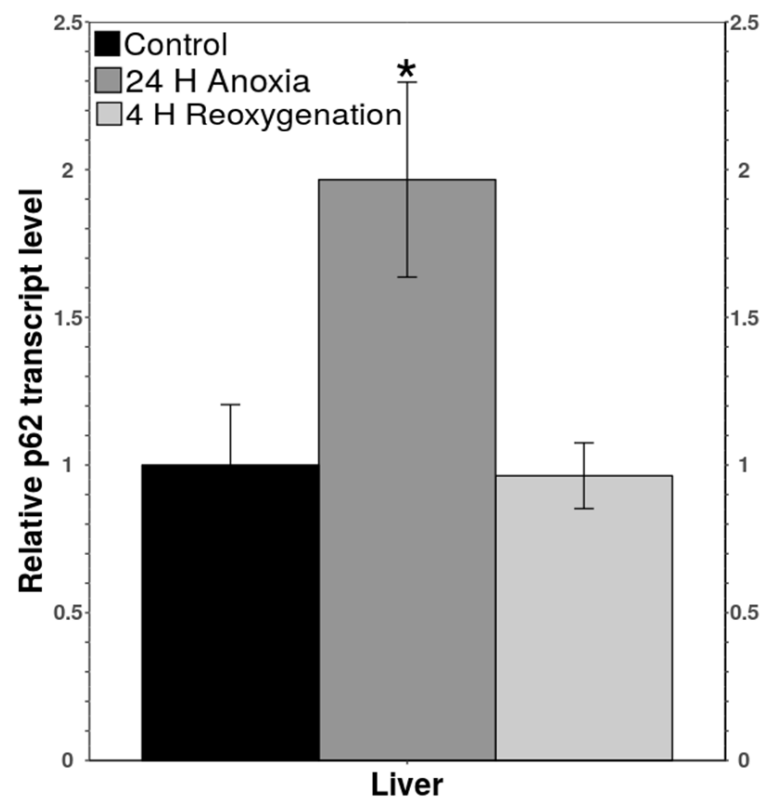

B

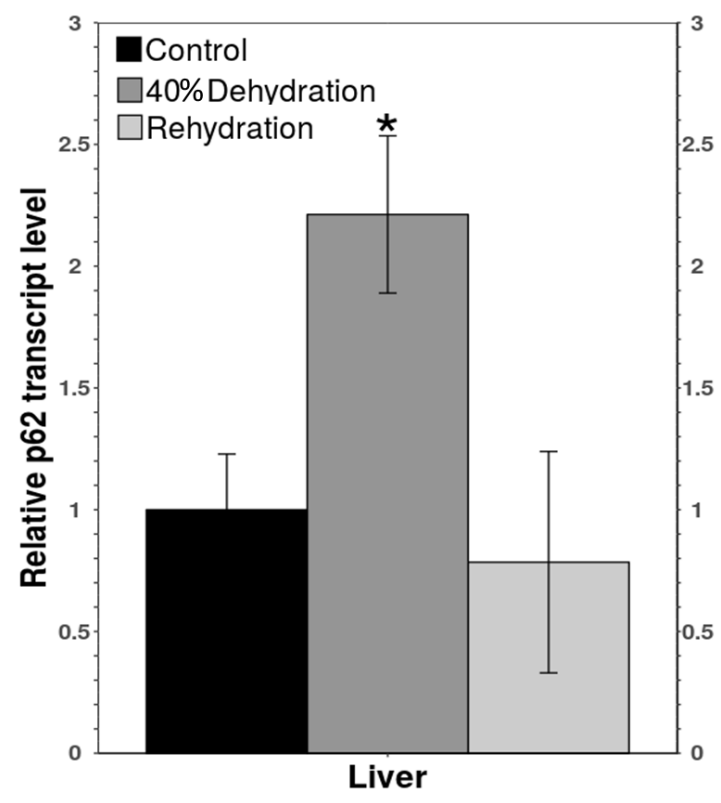

Figure 2.11: Relative transcript levels of p62 (sqstm1) in liver of wood frogs measured using RT-qPCR. Measurements were done using liver of wood frogs exposed to A) anoxia/reoxygenation, and B) dehydration/rehydration cycle. For more information, see Figure 2.2. 
A

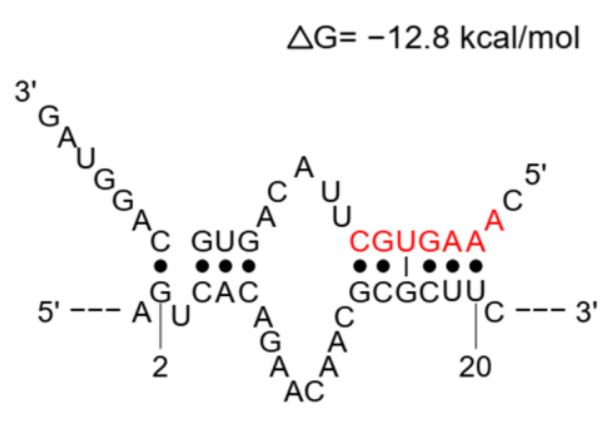

3'-UTR seed
B

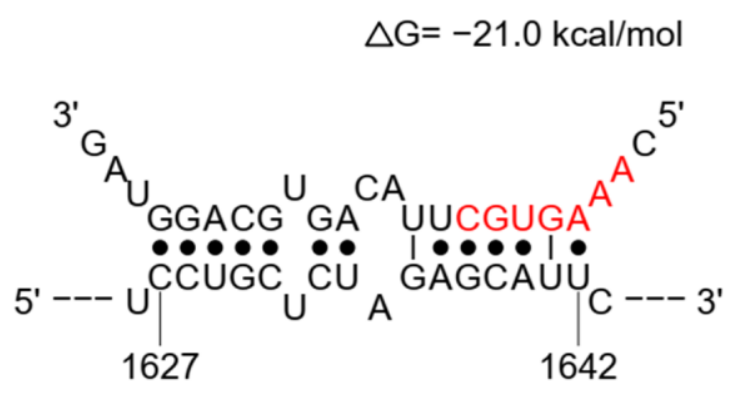

3'-UTR seedless

Figure 2.12: Schematic diagram showing the predicted binding sites of mmu-miR-17-5p to different regions of the $p 62$ transcript. The prediction of miR-17-5p binding to (A) seed-site and (B) seedless-site of the p62 (sqstm1) transcript was using the Xenopus laevis transcript sequence. The diagram was generated using the Sfold 2.2 STarMiR bioinformatics program. The seed-region (CGUGAAA) of mmu-miR-17-5p is shown in red. MiRNA binding to the seed-site showed a logistical probability of 0.579 with $\Delta \mathrm{G}=-12.8 \mathrm{kcal} \mathrm{mol}-1$, whereas binding to the seedless-site showed a logistical probability of 0.831 with $\Delta \mathrm{G}=-21.0 \mathrm{kcal} \mathrm{mol}-1$. 
Chapter 3: RAGE

management: ETS1- EGR1

mediated transcriptional

networks regulate

angiogenesis in wood frogs 
RAGE management: ETS1- EGR1 mediated transcriptional networks regulate angiogenesis in wood frogs

Rasha Al-attar ${ }^{1}$, Kenneth B. Storey ${ }^{1}$

${ }^{1}$ Institude of Biochemistry and Department of Biology, Carleton University, Ottawa, ON, Canada (K1S-5B6)

Corresponding Author:

Kenneth B. Storey

Kenstorey@cunet.carleton.ca

Telephone: +1 (613) 520-2600 x3678

Keywords: Angiogenesis, wood frogs, EGR1, ETS1, freeze/thaw, anoxia/reoxygenation, dehydration/rehydration, RAGE, AGE 


\subsection{Abstract}

Freeze-tolerant species, such as wood frogs (Rana sylvatica), are susceptible to multiple co-occurring stresses that they must overcome to survive. Freezing is accompanied by mechanical stress and dehydration due to ice crystal formation in the extracellular space, ischemia/anoxia due to interruption in blood flood, and hyperglycemia due to cryoprotective measures. Wood frogs can survive dehydration, anoxia, and high glucose stress independently of freezing, thereby creating a multifactorial model for studying freeze-tolerance. Oxidative stress and high glucose levels favors the production of prooxidant molecules and advanced glycation end product (AGE) adducts that could cause substantial cellular damage. In this study, the involvement of the high mobility group box 1 (HMGB1)-AGE/RAGE (receptor for AGE) axis and the regulation of ETS1 and EGR1mediated angiogenic responses were investigated in liver of wood frogs expose to freeze/thaw, anoxia/reoxygenation and dehydration/rehydration treatments. HMGB1 and not AGE-adducts are likely to induce the activation of ETS1 and EGR1 via the RAGE pathway. The increase in nuclear localization of both ETS1 and EGR1, but not DNA binding activity in response to stress hints to a potential spatial and temporal regulation in inducing angiogenic factors. Freeze/thaw and dehydration/rehydration treatments increase the levels of both pro- and anti-angiogenic factors, perhaps to prepare for the distribution of cryoprotectants or enable the repair of capillary damaged capillaries and wounds when needed. Overall, wood frogs appear to anticipate the need for angiogenesis in response to freezing and dehydration but not anoxic treatments, probably due to mechanical/architectural stresses associated with the two former conditions. 


\subsection{Introduction}

The freeze-tolerant wood frog, Rana sylvatica, is one of the few vertebrate species that can tolerate full body freezing in winter [1]. With over $65-70 \%$ of its total body water frozen in winter, these animals experience no breathing, no heartbeat, no brain conductivity or muscle movement but these functions resume to normal only hours after thawing [1]. Freezing is the integration of multiple stresses that occur simultaneously and must be adequately dealt with to ensure survival. For example, to prevent damage to intracellular organs and cell membrane integrity, wood frogs dehydrate their cells to confine ice crystal formation strictly to the extracellular and interstitial spaces [2]. As a result, wood frogs experience extreme dehydration, which is associated with increased osmolality, ionic strength, and substantial decrease in cell volume [3]. Moreover, freezing also interrupts blood delivery to organs as the heart stops beating and blood freezes, therefore wood frog organs are left anoxic [4]. Inadequate oxygen availability under these conditions initiates a biomolecular response that favors anaerobic metabolism, accompanied by a strong reduction in metabolic rate to match energy demand with supply [1]. The strict regulation of energy allocation allows for pro-survival pathways to be induced $[5,6]$ and processes that are energy expensive and potentially injurious to the cell to be reduced [7]. As a cryoprotective measure, wood frogs substantially increase their blood glucose levels from $5 \mathrm{mM}$ under normal conditions to $\sim 300 \mathrm{mM}$ during freezing and become extremely hyperglycemic [2]. Interestingly, wood frogs can survive anoxia, dehydration, and glucose-loading even when unfrozen, thereby providing a unique model for studying freezing and its associated stresses independently. 
With glucose used as a cryoprotective agent in response to freezing and dehydration $[3,8]$, glucose-induced cytotoxicity becomes imminent. Indeed, high levels of glucose could result in macromolecule glycation, a non-enzymatic process where a covalent bond is created between the free amino groups of amino acids $(\mathrm{N}$-terminal amino acids, lysine or arginine) and the carbonyl group of a reducing sugar (in this case glucose) in a process called the Maillard reaction to form a Schiff base [9]. After isomerization, the Schiff base is converted into a stable Amadori product. Fragmentation of these products through retro-aldol reactions generates a variety of carbonyl species that are highly reactive called glycation intermediaries. These intermediate molecules can undergo further oxidation, dehydration, and degradation reactions to form highly stable advanced glycation end products (AGEs) [10]. These AGE products can also react with amino acid residues to form AGE-protein adducts or protein crosslinks [10]. Carbonyl groups can also be generated during lipid catabolism (e. g. malondialdehyde), forming the advanced lipoxidation end products (ALEs) [10]. Although originating from different sources, AGEs and ALEs cannot always be distinguished from each other. For example, the most common AGE compound, N-e-carboxymethyllysine (CML) [11], can be generated following both lipid and carbohydrate catabolism [12]. Alternatively, excessive glucose in the presence of oxidative stress can undergo autoxidation and yield highly reactive carbonyl-containing compounds that can also be conjugated to proteins [10]. In short-lived proteins, glycation results in the formation of Amadori products only. This is because AGE formation is a very slow process and can take up to months or years; therefore, AGE formation predominantly affects long lived proteins such as 
collagen. However, hyperglycemia or oxidative stress can accelerate the formation of AGEs to hours, thereby also affecting short-lived macromolecules [13].

Previous study on wood frogs demonstrated that despite high levels of glucose during freezing, glycated hemoglobin levels decreased after 48 hours of freezing and levels continued to decrease following 3-7 days of thawing [14]. Interestingly, the same study reported an elevation in glycated serum albumin levels 7 days post-thawing, suggesting that perhaps wood frogs have a unique mechanism for selective protein glycation [14]. AGE adducts, along with other secretory molecules, can bind to the receptor for advanced glycation end products (RAGE) and initiate a cascade of intracellular events that results in the repression or induction of numerous signaling pathways [15]. RAGE is a cell surface receptor from the immunoglobulin superfamily, and it recognizes its ligands based on their three-dimensional structures, rather than specific amino acid epitopes [15]. Along with AGEs, the high mobility group box 1 (HMGB1) is another widely acknowledged ligand for RAGE [16]. Under normal conditions, this non-histone DNA binding protein resides in the nucleus, but upon stimulation with oxidative stress, it becomes acetylated and is secreted into the cytoplasm [17]. There, it can interact with other regulatory proteins to promote or repress numerous signaling processes. For example, in response to various stimuli, HMGB1 has been shown to promote autophagy by binding to and activating Beclin1, an important autophagy initiator [18]. HMGB1 can also translocate to the extracellular space via secretory vesicles and bind to various receptors including RAGE [19]. Association of AGEs and RAGE has been implicated in regulating inflammation, autophagy and angiogenesis amongst other processes [15,20]. Angiogenesis, in theory, could be a 
beneficial response to freezing and dehydration as it would speed up the delivery of cryoprotects such as glucose and urea to different organs. Interestingly, a cDNA array screen in our laboratory demonstrated an increase in RAGE levels in wood frog heart following freezing [21]. Therefore, it was of interest to study whether a) AGEs accumulate in wood frogs during freeze/thaw, anoxia/reoxygenation, dehydration/rehydration cycles and b) if so, can RAGE induce a transcriptional response under these conditions.

Binding of ligands to RAGE activates several kinases that ultimately lead to the activation of various transcription factors such as the E26 transformation-specific sequence 1 (ETS-1) and the early growth response 1 (EGR1) under conditions of hypoxia or oxidative stress $[22,23]$ (Figure 3.1). Upon ligand binding to the extracellular domain of RAGE, its cytoplasmic domain interacts with diaphanous-1 (mDia-1), a protein that is necessary to transduce signals to downstream kinases. Indeed, activation of mDia-1 was necessary for protein kinase $\mathrm{C} \beta \mathrm{II}$ activation and its subsequent phosphorylation of ERK1/2 and JNK under low oxygen conditions resulting in the phosphorylation and activation of the ELK-1 transcription factor [22,23]. This facilitates ELK-1 cytoplasmic to nuclear translocation, and induction of the egrl gene [24]. After being translated in the cytoplasm, EGR1 enters the nucleus and induces the transcription of various genes involved in tissue repair, inflammation and angiogenesis [25]. The transcriptional regulation by EGR1 depends on two co-repressors, NAB1 (NGFI-A binding-1, constitutive) and NAB2 (inducible), which physically interact with EGR1 to prevent its association with DNA and transcriptional activity [26,27]. The mechanism of ETS1 activation is similar to the mechanism of EGR1 activation. Following ERK1/2 activation, 
this kinase phosphorylates ETS1, a process that is necessary to facilitate its cytoplasmic to nuclear translocation [28].

Both EGR1 and ETS1 are involved in angiogenesis; the formation of new blood vessels stemming from pre-existing ones [29-31]. While angiogenesis is a normal physiological process during development and adulthood, its inappropriate initiation could manifest in serious pathology such as cancer [32]. By contrast, activation of angiogenesis in ischemic heart or during tissue injury has been shown reduce the progression of cardiac infarction [33], and accelerate would healing [34]. Under normal conditions, endothelial cells (ECs) are in their quiescent state and reside in the luminal surface of the vessels. Upon stimulation by inflammation or injury, several growth factors are released including but not limited to vascular endothelial growth factor (VEGF), transforming growth factors (TGFs), endothelial growth factors (EGFs), tumor necrosis factor $\alpha(\mathrm{TNF} \alpha)$, angiogenin, angiopoietin, and fibroblast growth factors (FGFs) [34,35]. These growth factors bind to receptors (e.g. VEGF receptors, FGF receptors, tyrosine kinase with immunoglobulin-like receptors, and EGF-like domain 1/2 receptors, Tie1, Tie2, and integrin receptors) found on the EC surface of the parent vessel, and promote the induction and release of proteolytic enzymes that degrade the basement membrane surrounding the parent vessel [35]. Following a chemotactic gradient toward growth factors, EC cells will proliferate and sprout until the two or more sprouts fuse together to form a lumen. Oxygenated blood with flow through the lumen to supply local tissue with oxygen, and when levels return to normal, growth factor secretion (mainly VEGF) decreases and returns to baseline. Pericytes are then recruited to promote the maturation and stabilization of the newly formed capillary. While angiogenesis is a complex process 
and requires a coordinated response from multiple signaling pathways, the underlying mechanism remains the same: to detect secreted pro-angiogenic ligands from secreting vessels by receptors found on the parent vessels. Here, the role of AGE/HMGB1/RAGE axis in regulating angiogenesis in response to environmental stressors in the wood frog was studied. Current results show that angiogenic responses are likely induced by the HMGB1/RAGE axis, and that ETS1 and EGR1 regulate the expression of angiogenic targets in a temporal manner in response to freezing and dehydration in frogs.

\subsection{Materials and methods}

Animal treatment

Animal treatment was performed as previously published [36,37]. Briefly, adult male wood frogs were captured at breeding ponds near Oxford Mills, Ontario and transported on ice to Carleton University's animal care facility. Following a tetracycline bath and a two-week acclimation period at $5^{\circ} \mathrm{C}$, the frogs were divided into 7 groups. The control group was sampled following the acclimation period, whereas the remaining groups ( $\sim 25$ frogs per group) destined for the freeze/thaw treatments were placed in plastic containers (3-4 frogs per container) previously lined with damp paper towel, and housed in incubators set to $-4^{\circ} \mathrm{C}$ for 45 minutes. This process is done to promote a gradual decrease in body temperature and initiate the ice nucleation process. Following incubation, the temperature of the incubator was increased to $-2.5^{\circ} \mathrm{C}$ and wood frogs were maintained at this temperature for 24 hours. This incubation period was chosen because it was previously reported that wood frogs reach maximum body ice content and exhibit no signs of breathing, movement, heartbeat, or brain conductivity following 24 hours freezing [38]. The frozen group were sampled at this time point, and the other frozen 
group was transferred to a $5^{\circ} \mathrm{C}$ incubator for the next 8 hours to facilitate wood frog thawing. This incubation period was chosen because wood frogs have been shown to regain most of their physiological functions at that time [38]. The thawed group was sampled at this time point. The anoxia/reoxygenation and dehydration/rehydration treatments were performed as previously published [36].

All animals were euthanized by pithing. Tissues were excised quickly and immediately flash frozen in liquid nitrogen to preserve the metabolic profile in each condition before storage at $-80^{\circ} \mathrm{C}$. All animal treatment protocols were approved by Carleton University's Animal Care Committee (protocol no. 13683), and all procedures were performed following the guidelines set by the Canadian Council on Animal Care.

\section{Total protein extraction}

Total protein extraction was performed as previously described [37]. Briefly, $\sim 50$ mg of frozen liver tissue from control, 24 hour freezing, 8 hour thaw, 24 hour anoxia, 4 hour reoxygenation, $40 \%$ dehydration and rehydration was homogenized 1:5 (w:v) with lysis buffer (EMD Millipore, catalogue no. 43-045) containing $1 \mathrm{mM} \mathrm{Na}_{3} \mathrm{VO}_{4}, 10 \mathrm{mM} \beta$ glycerophosphate, $10 \mathrm{mM} \mathrm{NaF}$, and $10 \mu \mathrm{L} / \mathrm{mL}$ of protease inhibitor cocktail (Bioshop, catalogue no. PIC001.1) using a Polytron homogenizer for $\sim 20$ seconds. All samples were placed on ice for $\sim 40$ minutes with intermitted vortexing every 10 minutes. Samples were then centrifuged at $10,000 \mathrm{xg}$ at $4^{\circ} \mathrm{C}$ for 20 minutes, and the supernatant containing the soluble protein fraction was collected. The total protein concentration of all samples was measured using the Bradford method (Bio-Rad, catalogue no. 500-0006), and the concentration was adjusted to $10 \mu \mathrm{g} / \mu \mathrm{L}$ before storage at $-80^{\circ} \mathrm{C}$ using the respective cytoplasmic and nuclear extraction buffers. 


\section{Cytoplasmic-nuclear fractionation}

To assess the localization of transcription factors and other secretory proteins, a cytoplasmic-nuclear fractionation procedure was performed. Approximately $50 \mathrm{mg}$ of frozen tissue was homogenized 1:5 (w:v) with 1x buffer A (10 mM HEPES, $10 \mathrm{mM} \mathrm{KCl,}$ $10 \mathrm{mM}$ EDTA, $20 \mathrm{mM} \beta$-glycerol phosphate (pH 7.9), $10 \mu \mathrm{L}$ of $100 \mathrm{mM}$ DTT per mL of buffer and $10 \mu \mathrm{L}$ of protease inhibitor cocktail per $\mathrm{mL}$ of buffer) using a Dounce homogenizer for a total of $\sim 3-4$ stokes. Samples were then incubated on ice for 25 minutes to allow for adequate extraction before centrifugation at $12,000 \mathrm{rpm}$ for 15 minutes at $4{ }^{\circ} \mathrm{C}$. The supernatant was collected and stored as the cytoplasmic fraction.

The pellets were resuspended in $\sim 300 \mu \mathrm{L}$ of $5 \mathrm{X}$ buffer B (100 mM HEPES, $2 \mathrm{M}$ $\mathrm{NaCl}, 5 \mathrm{mM}$ EDTA; $50 \%$ (v:v) glycerol, $100 \mathrm{mM} \beta$-glycerol phosphate (pH 7.9), $10 \mu \mathrm{L}$ of $100 \mathrm{mM}$ DTT and $10 \mu \mathrm{L}$ of protease inhibitor per $\mathrm{mL}$ of buffer), and sonicated for 10 seconds. All samples were incubated on ice for 10 minutes, before being centrifuged at $14,000 \mathrm{rpm}$ for 10 minutes at $4{ }^{\circ} \mathrm{C}$. The supernatant was collected and stored at $-80^{\circ} \mathrm{C}$ as the nuclear fraction.

The concentration of both cytoplasmic and nuclear fraction was measured using the Bradford method, and the sample concentration was normalized to $5 \mu \mathrm{g} / \mu \mathrm{L}$. The successfulness of the fractionation procedure was determined by western blotting, where the enrichment of $\beta$-tubulin (cytoplasmic marker) and histone $\mathrm{H} 3$ (nuclear marker) in the cytoplasmic and nuclear fractions respectively was demonstrated in the respective fractions.

Western blotting 
The concentration of total proteins as well as cytoplasmic and nuclear fractions for all samples was measured using a Bradford assay and standardized to a constant concentration immediately before the start of protein treatment. All samples were mixed with equal volumes of $2 \mathrm{X}$ SDS loading buffer $(0.2 \% \mathrm{w}: \mathrm{v}$ bromophenol blue, $4 \% \mathrm{w}: \mathrm{v}$ SDS, $10 \%$ v:v 2- $\beta$-mercaptoethanol, $20 \% \mathrm{v}: \mathrm{v}$ glycerol and $100 \mathrm{mM}$ Tris-base $\mathrm{pH} 6.8$ ), and boiled for 5 minutes in a water bath. Subsequently, samples were cooled on ice and stored at $-20^{\circ} \mathrm{C}$ for future use.

The western blot procedure was performed as previously described [37]. The following antibodies were purchased from Abclonal: Ac-HMGB1 K29 (A16002), NAB1 (A15692), NAB2 (A5827), FGF2 (A0235), ANGPT1(A7877), ANGPT2 (A0698), Tie1(A3334), Tie2(A0743), VEGFR1, VE-cad (A0734). ETS1(Sc-55581) and EGR1 (Sc-189) antibodies were purchased from Santa-Cruz biotechnology. RAGE(GTX23611) and VEGF(GTX129844) antibodies were purchase from GeneTex and the HMGB1(PCRP-HMGB1-3A7) antibody was purchase from the University of Iowa hybridoma bank.

Advanced glycation end-product (AGE) measurement

Wood frogs are exposed to high levels of hyperglycemia in response to freezing and dehydration, and this could promote protein glycation resulting in RAGE activation. Therefore, total levels of AGEs formed under each condition was measured using a commercially available OxiSelect ${ }^{\mathrm{TM}}$ Advanced Glycation End Product (AGE) Competitive ELISA Kit (Cell BioLabs, catalogue no. STA-817) following the manufacturer's protocol. All reagents except for phosphate buffered saline (137 mM $\mathrm{NaCl}, 10 \mathrm{mM} \mathrm{Na}_{2} \mathrm{HPO}_{4}, 2.7 \mathrm{mM} \mathrm{KCl}$, and $2 \mathrm{mM} \mathrm{KH}_{2} \mathrm{PO}_{4}, \mathrm{pH}$ 7.4) were provided by the 
manufacturer. AGE conjugates $(10 \mu \mathrm{g} / \mathrm{mL})$ were diluted in PBS and mixed in a 1:1 ratio with $1 \mathrm{x}$ conjugate diluent also diluted in PBS. A volume of $100 \mu \mathrm{L}$ of the resulting mixture was added to each well, and the plate was incubated at $4^{\circ} \mathrm{C}$ overnight without agitation. Following incubation, excess AGE conjugates were decanted, and the wells were washed twice with $1 \mathrm{X}$ PBS. To block unreactive sites, $200 \mu \mathrm{L}$ of assay diluent was added to each well and the plate was blocked at room temperature for 1 hour. Following incubation, excess assay diluent was discarded and $50 \mu \mathrm{L}$ of total liver extracts $(55 \mu \mathrm{g})$ diluted with $1 \mathrm{X}$ PBS containing $0.1 \%$ BSA, or AGE-BSA standard was added to each well in duplicate. The plate was incubated for 10 minutes at room temperature on a rocker, before the addition of $50 \mu \mathrm{L}$ of anti-AGE antibody to each well. The plate was incubated at room temperature for 1 hour while rocking and subsequently washed $3 \mathrm{x}$ with $250 \mu \mathrm{L}$ of wash buffer. A volume of $100 \mu \mathrm{L}$ of secondary antibody conjugated to HRP was added to each well and the plate was incubated for 1 hour at room temperature while rocking. Following incubation, the wells were washed three times and incubated with 100 $\mu \mathrm{L}$ of substrate solution for $\sim 10$ minutes at room temperature while rocking. Following color development, the reaction was stopped by adding $100 \mu \mathrm{L}$ of stop solution to each well and the plate was read at $450 \mathrm{~nm}$. The amount of AGE protein adducts in the sample was calculated using the standard curve.

DNA-protein interaction enzyme linked immune sorbent assay (DPI-ELISA)

To assess the DNA binding activity of ETS1 and EGR1 to their consensus sequence, a DPI-ELISA technique using DNA probes specific to these transcription factors was performed following a previously established method [39]. DNA oligonucleotides containing the consensus sequence of ETS1 (forward, 5'-Biotin- 
GATCTCGAGCAGGAAGTTCGA-3',

CTAGAGCTCGTCCTTCAAGCT-5') and EGR1 (forward- 5'-Biotin GGATCCAAGCGGGGGCGAGCGGGGGCGA-3', complementary $\quad 3{ }^{\prime}-$ CCTAGGTTCGCCCCCGCTCGCCCCCGCT-5') were purchased from Integrated DNA Technologies (Coralville, IA, USA). The concentration of 1:1000 was used for anti-ETS1 and anti-EGR1 antibodies and a concentration of 1:2000 anti-rabbit-HRP antibody was used in this experiment. Probe specificity for each transcription factor was tested by performing the same assay but omitting either the probes, primary antibody, or proteins from the reaction. Varying concentration of pooled samples was used to determine the optimum protein concentration for each transcription factor under each condition.

\section{Total RNA isolation and cDNA synthesis}

Total RNA extraction was carried out as previously described [40]. First strand cDNA synthesis and mRNA quantification was done following a previously established protocol [41]. In a final volume of $10 \mu \mathrm{L}, 1 \mu \mathrm{g}$ of RNA and $1 \mu \mathrm{L}$ of $200 \mathrm{ng} / \mu \mathrm{L}$ Oligo (DT) (Sigma Genosys, TX, USA) were combined and incubated in a thermocycler (iCycler, Bio-Rad) set to $65{ }^{\circ} \mathrm{C}$ for 5 minutes. Samples were cooled on ice for few minutes, combined with $1 \mu \mathrm{L}$ of dNTP (BioShop), $1 \mu \mathrm{L}$ of MMLV Reverse Transcriptase (Invitrogen), $2 \mu \mathrm{L}$ of $100 \mathrm{mM}$ DTT and $4 \mu \mathrm{L}$ of $5 \mathrm{x}$ First Strand Buffer (Invitrogen), and incubated for 60 minutes at $42{ }^{\circ} \mathrm{C}$. Following cDNA synthesis, all samples were cooled on ice and serially diluted before storage at $-20{ }^{\circ} \mathrm{C}$.

The forward and reverse primers for ets 1 were designed using a conserved region of the coding sequence found in several species and purchased from Integrated DNA Technologies. For transcript quantification, $18 \mu \mathrm{L}$ of master mix $\left(10.12 \mu \mathrm{L}\right.$ of $\mathrm{ddH}_{2} \mathrm{O}, 4$ 
$\mu \mathrm{L}$ of $1 \mathrm{M}$ Trehalose, $0.5 \mu \mathrm{L}$ of $100 \%$ formamide, $0.5 \mu \mathrm{L}$ of forward and $0.5 \mu \mathrm{L}$ of reverse primers $(0.3 \mathrm{nmol} / \mu \mathrm{L}), 0.16 \mu \mathrm{L}$ of $25 \mathrm{mM}$ dNTPs, $0.125 \mu \mathrm{L}$ of $5 \mathrm{U} / \mu \mathrm{L}$ Taq Polymerase (BioShop, catalogue no. TAQ001.1), $0.1 \mu \mathrm{L}$ of 100x SYBR Green diluted with DMSO, and $2 \mu \mathrm{L}$ of $10 \mathrm{X}$ q-RT-PCR buffer $(500 \mathrm{mM} \mathrm{KCl,} 100 \mathrm{mM}$ Tris- $\mathrm{HCl} \mathrm{pH}$ 8.5, $20 \mathrm{mM} \mathrm{MgCl} 2,2 \mathrm{mM}$ dNTP, $1.5 \%$ Triton X-100 and $100 \mathrm{nM}$ Fluorescein) was added to $2 \mu \mathrm{L}$ of cDNA, and amplification was carried out using the CFX Connect ${ }^{\mathrm{TM}}$ Real-Time PCR Detection System (Bio-Rad, cat. No. 1855201) according to MIQE guidelines [42]. The amplification protocol for ets 1 is as follows: $95{ }^{\circ} \mathrm{C}$ for 3 minutes, 39 cycles of $95{ }^{\circ} \mathrm{C}$ for 20 seconds, $56.7{ }^{\circ} \mathrm{C}$ for 20 seconds and $72{ }^{\circ} \mathrm{C}$ for 20 seconds. The amplification protocol for the reference gene, $\beta$-actin, was performed according to previously optimized parameters [43]. The following primers were used: $\beta$-actin Forward (5'AGAAGTCGTGCCAGGCATCA-3'), Reverse (5'-AGGAGGAAGCTATCCGTGTT3') and ets 1 Forward (5'-ATCAAGCAGGAGGTGGTGTC-3') And Reverse (5'GATTGGTCCACTGCCTGTGT-3').

\section{Quantification and statistics}

For western blot measurements, band intensities obtained from the enhanced chemiluminescent signal and the corresponding Coomassie Blue-stained membranes were analyzed using GeneTools (Syngene, Frederick, MD, USA). To account for loading variation, band densities from the enhanced chemiluminescent signal were standardized against a group of stably expressed proteins within the same lane. This method of analysis is more reliable than using a single housekeeping protein [44]. Fold changes in response to stress and recovery are reported relative to the control group (set to 1). Absorbance values from DPI-ELISA and AGE measurement were measured at $450 \mathrm{~nm}$, 
with $655 \mathrm{~nm}$ set as the reference wavelength. Relative DNA binding of ETS1 and EGR1 under different conditions are reported relative to the control, which was set to 1 . Values obtained from AGE measurement were converted to protein amount using an AGE-BSA standard curve and are reported as levels of AGE adducts for each condition. Relative mRNA transcript levels were calculated by linearizing the raw $\mathrm{Cq}$ values $\left(2^{-\mathrm{Cq}}\right)$, and ets 1 values were standardized against values obtained for $\beta$-actin. Fold-change during stress and recovery are reported relative to the control, which was set to 1 . Histograms for all experiments represent the mean relative protein expression (mean $\pm \mathrm{SEM}$ ) measured in $\mathrm{n}=4$ independent biological replicates. Statistically significant difference compared to the control $(\mathrm{p}<0.05)$ were calculated using a One-Way ANOVA and analyzed using the Dunnett's post-hoc test. All statistical analyses and graphical presentations were performed using the RBIOPLOT statistical and graphical R package [45].

\subsection{Results}

\section{Relative expression levels of RAGE and its ligands}

Protein levels of RAGE, HMGB1 and Ac-HMGB1 (K29) were measured using western blotting in liver of wood frogs undergone freeze/thaw, anoxia/reoxygenation and dehydration/rehydration treatments. For freeze/thaw experiments, total levels of HMGB1 (T-HMGB1) were unchanged in response to freezing but increased by $2.8 \pm 0.6$-fold after thawing (Figure 3.2A). Levels of acetylated HMGB1 (Ac-HMGB1 K29), the cytoplasmic form of HMGB1, increased by $1.4 \pm 0.07$-fold following freezing but returned to baseline after thawing. Protein levels of RAGE rose by $2.7 \pm 0.7$-fold after freezing, and showed a reduction following an $8 \mathrm{H}$ thaw, although the decrease was not deemed statistically significant. 
Anoxia/reoxygenation treatment results demonstrate that T-HMGB1 is significantly increased by $1.5 \pm 0.1$-fold following anoxia exposure, but levels returned to control values after reoxygenation (Figure 3.2B). Levels of Ac-HMGB1 K29 remained unchanged in response to treatment, whereas levels of RAGE significantly rose by $2.5 \pm$ 0.2-fold and $2.3 \pm 0.14$-fold following anoxia/reoxygenation treatment. Dehydration/rehydration treatment showed a similar response as anoxia/reoxygenation treatment. Here, levels of T-HMGB1 rose by $1.8 \pm 0.1$-fold in response to dehydration before decreasing to near control values after rehydration (Figure 3.2C). Like previous results, dehydration/rehydration had no effects on HMGB1 acetylation, whereas levels of RAGE rose by $10.9 \pm 0.7$-fold and $7.6 \pm 0.9$-fold, respectively.

Total levels of AGE in liver from different conditions was measured using a commercially available kit and the amount of AGE-adduct was calculated based on a BSA-AGE standard curve. Under normal conditions, wood frog liver had $4.4 \pm 0.5$ $\mu \mathrm{g} / \mathrm{mL}$ of AGE adducts. This level decreased significantly down to $2.3 \pm 0.2 \mu \mathrm{g} / \mathrm{mL}$ and $2.7 \pm 0.6 \mu \mathrm{g} / \mathrm{mL}$ in response to freezing and thawing (Figure 3.3). In response to anoxia, levels decreased to $1.8 \pm 0.2 \mu \mathrm{g} / \mathrm{mL}$ but rebound to near control values $(4.7 \pm 0.2 \mu \mathrm{g} / \mathrm{mL})$ after reoxygenation. A similar response was observed during dehydration where levels decreased to $2.2 \pm 0.2 \mu \mathrm{g} / \mathrm{mL}$ but rebound to near control values after rehydration ( $4.4 \pm$ $0.5 \mu \mathrm{g} / \mathrm{mL})$.

Relative expression levels of ETS1 and EGR1

Using qRT-PCR, transcript levels of ets 1 were measured across all conditions. Relative to the control, transcript levels of ets 1 rose by $1.7 \pm 0.2$-fold and by $2.0 \pm 0.2$ fold after freezing and thawing respectively (Figure 3.4A). The same pattern was seen in 
response to anoxia and reoxygenation where transcript levels rose by $2.1 \pm 0.3$-fold and by $2.0 \pm 0.3$-fold, respectively (Figure 3.4B). In contrast, transcript levels of ets 1 decreased significantly by $91 \pm 1.4 \%$ and by $84 \pm 2.3 \%$ in response to dehydration and rehydration, respectively (Figure 3.4C).

Using western blotting, the levels of ETS1 and EGR1, two transcription factors that are activated downstream of RAGE and are involved in angiogenesis were measured $[29,30,46]$. Relative to the control, total protein levels of ETS1 increased significantly by $3.5 \pm 0.6$-fold in response to freezing but returned to normal upon thawing (Figure 3.5A). EGR1 protein levels rose by $2.1 \pm 0.17$-fold after freezing and increased further by $3.2 \pm$ 0.4-fold after thawing. Anoxia and reoxygenation had no influence on total protein expression of ETS1 or EGR1 (Figure 3.5B). In response to dehydration, levels of ETS1 remained the same but decreased by $58 \pm 3 \%$ after rehydration. Dehydration and rehydration treatments did not appear to affect the total protein expression of EGR1 in liver (Figure 3.5C).

Relative nuclear localization level of ETS1, EGR1 and two repressors of EGR1

Following cytoplasmic and nuclear fractionation, the nuclear fraction of all conditions was used in western blot analysis to assess the relative nuclear localization of ETS1, EGR1, NAB1 and NAB2, where the latter two proteins are EGR1 repressors. In response to freezing, the nuclear localization of ETS1 increased by $2.3 \pm 0.6$-fold but returned to near control values after thawing (Figure 3.6A). Levels of EGR1 rose significantly in response to freeze/thaw treatment by $3.0 \pm 0.5$-fold and by $3.1 \pm 0.6$-fold, respectively. Nuclear levels of NAB1 increased by $1.4 \pm 0.04$-fold in response to freezing 
but decreased by $37 \pm 9 \%$ after thawing. NAB2 levels also increased by $1.9 \pm 0.2$-fold after freezing but returned to near control values after thawing.

Anoxic treatment increased ETS1 nuclear localization by $1.4 \pm 0.1$-fold, but levels returned to normal after reoxygenation (Figure 3.6B). There was no significant change in nuclear localization levels of EGR1 or NAB1, but NAB2 levels increased by $2.1 \pm 0.2$ fold and by $3.5 \pm 0.4$-fold respectively following anoxia and reoxygenation. In response to dehydration and rehydration treatment, nuclear accumulation levels of ETS1 increased by $2.3 \pm 0.3$-fold and by $2.1 \pm 0.3$-fold (Figure 3.6C), whereas the same treatment did not show a statistically significant change in EGR1 nuclear levels. Nuclear levels of NAB1 decreased by $39 \pm 6.1 \%$ and by $37 \pm 7.3 \%$ in response to dehydration and rehydration, respectively. For NAB2, exposure to dehydration did not have an effect on its nuclear localization, but rehydration treatments reduced its levels by $67 \pm 7.1 \%$.

\section{DNA binding activity of ETS1 and EGR1}

To determine whether ETS1 and EGR1 were transcriptionally active, a DPIELISA was performed. Freezing and thawing treatments resulted in a significant reduction in ETS1 DNA binding activity by $53 \pm 4.5 \%$ and $50 \pm 11 \%$ respectively, whereas no effect was seen on EGR1 DNA binding in response to either treatment (Figure 3.7A). Similar results were obtained after anoxia/reoxygenation treatment where relative ETS1 DNA binding activities decreased by $81 \pm 5 \%$ and by $61 \pm 6 \%$ respectively. (Figure 3.7B), The same treatment had no effect on EGR1 DNA binding. Dehydration also resulted in a significant decrease in ETS1 DNA binding by $53 \pm 7 \%$, but levels return to near normal after rehydration (Figure 3.7C). In response to both 
dehydration and rehydration, levels of EGR1 DNA binding increased by $1.4 \pm 0.05$-fold and by $1.7 \pm 0.1$-fold, respectively.

Protein expression levels of angiogenic factors regulated by ETS1 and EGRI

Using western blotting, protein expression levels of select angiogenic markers previously reported to be under the transcriptional control of ETS1 and EGR1 were measured. In response to freezing and thawing, protein levels of VEGF remained the same, whereas levels of FGF2 decreased by $80 \pm 4.3 \%$ after freezing but increased by 1.8 \pm 0.1 -fold after thawing, relative to the control group (Figure 3.8). Protein levels of ANGPT1 increased by $1.9 \pm 0.2$-fold after freezing but returned to baseline after thawing, whereas levels of ANGPT2 increased in response to both treatments by $1.5 \pm 0.03$-fold and by $1.8 \pm 0.2$-fold, respectively. Levels of Tie1 showed no statistical change in response to freezing but increased by $2.0 \pm 0.05$-fold after thawing. Similar trends were seen in Tie2 protein levels where a $5.1 \pm 0.3$-fold and $3.9 \pm 0.3$-fold increase was seen in response freezing and thawing, respectively. Protein levels of VEGFR1 also increased by $3.3 \pm 0.3$-fold after freezing but returned to near control values after thawing, whereas levels of VE-cad increased in response to both treatments by $1.3 \pm 0.1$-fold and $1.3 \pm 0.1$ fold, respectively.

Anoxia and reoxygenation treatment showed a slightly different response (Figure 3.9). In response to anoxia and reoxygenation, protein levels of VEGF decreased by $34 \pm$ $9 \%$ and by $41 \pm 5 \%$ respectively, relative to the control. There were no statistically significant changes in FGF2, ANGPT1 or VEGFR1 in response to either treatment. Levels of ANGPT2 did not show a significant decrease after anoxia treatment, but levels decreased significantly by $34 \pm 5 \%$ in response to reoxygenation. Total levels of Tiel 
increased in response to both treatments by $2.4 \pm 0.4$-fold and by $4.3 \pm 0.2$-fold, respectively. Tie2 protein levels increased by $1.8 \pm 0.1$-fold after anoxia treatment and further by $2.1 \pm 0.1$-fold after reoxygenation. Levels of VE-cad did not show a statistically significant change in response to anoxia, but levels decreased by $72 \pm 5 \%$ after reoxygenation.

Dehydration and rehydration treatment, to a certain degree, showed similar responses as the freeze/thaw experiments. Levels of VEGF were not affected by dehydration but decreased by $59 \pm 5 \%$ in response to rehydration (Figure 3.10). No significant changes were seen in FGF2 or VEGFR1 levels in response to either condition. Levels of ANGPT1 increased by $2.5 \pm 0.2$-fold after dehydration and by $2.0 \pm 0.04$-fold after rehydration. Similar results were observed in ANGPT2 where levels increased by $2.0 \pm 0.1$-fold and by $1.5 \pm 0.2$-fold in response to dehydration and rehydration, respectively. Levels of Tie1 increased by $3.8 \pm 0.3$-fold and by $2.6 \pm 0.5$-fold in response to dehydration and rehydration, respectively. Tie2 protein levels also showed the same trends where levels increased by $2.3 \pm 0.2$-fold and by $1.7 \pm 0.2$-fold in response to the dehydration, rehydration treatments. VE-cad levels increased significantly by $1.3 \pm 0.08$ fold in response to dehydration but returned to baseline values after rehydration.

\subsection{Discussion}

Wood frogs are resilient to many environmental stresses and can be used as model organisms to decipher the key molecular events in many complications associated with oxidative stress. Here, the involvement of the HMGB1/RAGE/AGE axis in promoting transcriptional responses to angiogenesis was studied in liver of wood frogs exposed to freeze/thaw, anoxia/reoxygenation and dehydration/rehydration treatments. HMGB1, a 
pro-inflammatory molecule, is predominantly a nuclear protein and is only secreted to the cytoplasm or extracellular space upon undergoing posttranslational modification in response to stress [17]. Among other posttranslational modifications, acetylation of HMGB1 was shown to be necessary for its nuclear export. Indeed, a single acetylation event at either of HMGB1's two nuclear localization signals can promote its nuclear export and cytoplasmic accumulation [17,47]. In addition to participating in multiple cytoplasmic events [48], and binding to extracellular receptors [49], HMGB1 has been studied extensively for its interaction with RAGE in hyperglycemic or pro-oxidative stress conditions, since this interaction can promote numerous downstream events such as inflammation, tissue repair, and angiogenesis [50-52]. In this study, total levels of liver HMGB1 showed an upwards trend in the 24 hour frozen condition, but this change was not statistically significant with respect to the control group (Figure 3.2A) Instead, HMGB1 levels rose significantly after recovery (Figure 3.2A). Interestingly, levels of acetylated HMGB1 (K29) increased significantly following freezing and returned to near control values after recovery. K29 is located within the nuclear localization signal and a single acetylation event on this residue has been shown to promote nuclear export [17]. Cytoplasmic HMGB1 can be exported to the extracellular space in two ways: 1) via pyroptosis, a proinflammatory program of cell death [53] or 2) via engulfment in secretory lysosome that will exocytose with cell membrane [19]. Given that wood frogs experience no sign of apoptosis under stress [7], it is logical to assume that acetylated HMGB1 probably reaches the extracellular membrane by exocytosis of secretory lysosomes. In correlation with HMGB1 acetylation, levels of RAGE also significantly increased in response to freezing before returning to near normal values after thawing 
(although a decreasing trend is observed, the change was not statistically significant). The increase in total HMGB1 but not in its acetylated form in response to thawing suggests that perhaps HMGB1 is retained in the nucleus. HMGB1 plays a significant role in maintaining the dynamics of the chromatin structure, transcription, and DNA repair. As such, its increase during thawing and probably nuclear retention is perhaps due to its nuclear specific functions [54]. Altogether, this suggests that perhaps acetylated HMGB1 following freezing associates with RAGE to induce downstream regulatory signals.

HMGB1 and RAGE responses to anoxia/reoxygenation (Figure 3.2B) and dehydration/rehydration (Figure 3.2C) were similar. In both cases, total levels of HMGB1 showed a significant increase during stress but returned to control levels during recovery; HMGB1 levels remained the same and RAGE levels increased following both stresses and both recoveries. Given that HMGB1 can be hyperacetylated at multiple sites, it is possible that in response to anoxia/reoxygenation and dehydration/rehydration cycles, a site other than K29 was acetylated [17]. Indeed, studies have shown that hypoxia or ischemia/reperfusion in hepatocytes promotes HMGB1 acetylation and its nuclear export [55]. Moreover, differential phosphorylation and methylation, along with changes in the redox state of HMGB1 have also been implicated in its nuclear export [56,57]. Reduced HMGB1 has been shown to bind to RAGE, whereas oxidized HMGB1 was shown to increase cytotoxicity and apoptosis [58]. Taking these studies into consideration, it is possible that posttranslational modifications other than acetylation and changes to HMGB1 redox status may regulate its translocation from the nucleus to the cytoplasm or extracellular space in anoxic or dehydrated wood frog liver. The increase in RAGE proteins in response to anoxia/reoxygenation and dehydration/rehydration treatments was 
also expected (Figure 3.2 B, 3.2 C respectively), as previous studies have reported the involvement of hyperglycemia and oxidative stress in promoting the expression of this receptor [59].

The experiments that measured the total levels of AGE adduct showed interesting results (Figure 3.3). In response to freeze/thaw treatment, anoxia and dehydration, total levels of AGE-adducts decreased significantly but returned to baseline after reoxygenation and rehydration. This was unexpected, as freezing, thawing and dehydration are accompanied by extreme hyperglycemia $[3,8]$. A previous study in wood frogs showed that levels of glycated serum albumin were unchanged in response to freezing and only increased 7 days post thawing [14]. Although speculative, it is possible that wood frogs show differential glycation patterns with respect to the nature of the protein. It is also possible that the duration of treatments in this study were not long enough to induce AGE formation. Indeed, the first step of AGE formation is the production of Schiff bases (reversible) and Amadori products, which are often short-lived [60]. Moreover, given the strong antioxidant capacity of wood frogs, it is possible that enhanced antioxidant responses during treatment prevent the formation or accumulation of AGEs. Antioxidant metabolites and antioxidant enzymes including superoxide dismutase, catalase, glutathione peroxidase, as well as carbonyl metabolizing enzymes such as aldehyde dehydrogenase, aldo-keto reductase, carbonyl reductase and glutathione S-transferases play a significant role in AGE removal [61]. Indeed, levels of aldo-keto reductases, glutathione S-transferases, and other antioxidants are significantly upregulated in response to stress in wood frogs $[6,62,63]$. As such, it is possible that the antioxidant response generated during stress and early hours of recovery, neutralizes, 
and/or prevents the formation of AGEs in wood frogs. Although levels are significantly decreased across different conditions, AGE-adducts exist in detectable levels in the cell; therefore, it is possible that even the limited number of AGE-adducts are enough to elicit a RAGE response.

Activation of RAGE by its ligands results in the induction and activation of the ETS1 and EGR1 transcription factors, and both are involved in the early stress response $[22,23]$. Transcript levels of ets 1 increased in response to freezing and thawing in wood frogs (Figure 3.4A). Protein levels of ETS1 and EGR1 rose significantly in response to freezing, and levels of EGR1 remained high even after thawing (Figure 3.5A), a trend that correlates with RAGE activation during freezing. While levels of ets 1 in response to freezing correlate with its protein levels, there is a discrepancy between the two levels after thawing. This is perhaps because high levels of the ETS1 transcription factor are not required after thawing, and that ETS1 activity is regulated by other mechanisms such as posttranscriptional modifications. The discrepancy between the transcript levels and protein levels after thawing could be due to regulation by microRNAs. MicroRNAs are small non-coding, single-stranded RNA species that bind to the transcript and prevent its translation [64]. Indeed, miR-1 and miR-499 were reported to suppress the translation of ets- 1 by regulating its sequestration away from the translational machinery or degradation [65]. Therefore, it is likely that the discrepancy seen in this study is due to regulation of the ets 1 transcripts at the posttranscriptional level. The increase in EGR1 total protein levels, despite unchanging levels of RAGE during thawing, could be due to its accumulation during freezing or early stages of thawing. Indeed, the transcription of egrl is mediated by ELK-1, and is dependent on JNK-mediated ELK-1 phosphorylation and 
nuclear localization [24]. In fact, JNK activity increased 90 minutes after thawing in wood frog liver [66]. Therefore, it is possible that induction of JNK activity in early hours of freezing is associated with EGR1 expression later stages of thawing as seen in the current study.

Transcript levels of ets 1 in response to anoxia/reoxygenation treatments showed the same response as exposure to freezing/thawing, where levels under both conditions increased significantly (Figure 3.4B). However, ets 1 transcript levels were significantly decreased in response to dehydration and rehydration (Figure 3.4C). There was no change in levels of ETS1 or EGR1 in response to anoxia/reoxygenation treatments (Figure 3.5B). By contrast, in response to dehydration, EGR1 remained the same and ETS1 levels decreased after rehydration (Figure 3.5C). It appears that ets 1 translation is also regulated at the posttranscriptional level in response to anoxia/reoxygenation, perhaps to provide the cell with a reservoir of ETS1 proteins when needed. However, in response to dehydration, ETS1 transcription factors appear to be regulated by posttranslational modifications or subcellular localization, and not at the transcriptional level. Given that RAGE transactivates ETS1 via phosphorylation, it is likely that perhaps ETS1 phosphorylation status is elevated under those conditions to facilitate its nuclear translocation. Indeed, JNK, one of the kinases involved in ETS1 phosphorylation was shown to be elevated in response to short- and long-term anoxia in liver of hatching turtles [67]. Similarly, ERK2, another kinase that phosphorylates and activates ETS1 is also upregulated in response to early dehydration in liver of Xenopus laevis [68]. Therefore, it is possible that an increase in kinase activity in early hours of dehydration is responsible for translocating ETS1 to the nucleus. 
Wood frogs and other hypometabolic species induce the expression of specific transcription factors when conditions are optimal, before the full onset of freezing or long-term anoxia/rehydration but maintain these factors in their inactive form until they are needed. This can be achieved by reversible protein modifications, a regulatory mechanism that is widely used in animals that undergo metabolic rate depression, which not only regulates enzymatic activities, but also controls subcellular localization of proteins [69]. As such, nuclear levels of ETS1, EGR1 and two EGR1 co-repressors were analyzed to determine whether this mode of regulation is used by these factors. In response to freezing, nuclear levels of ETS1 increased significantly but rebounded to control values after thawing (Figure 3.6A). This trend was similar to HMGB1-RAGE activation under the same conditions. On the other hand, the levels of EGR1 were elevated after both freezing and thawing, whereas levels of NAB1 increased after freezing but decreased after thawing. Moreover, levels of NAB2 also increased following freezing, but returned to normal after thawing (Figure 3.6A). An increase in nuclear ETS1 and EGR1 levels is consistent with RAGE-mediated activation of these two transcription factors. Both ETS1 and EGR1 are early response genes and are either induced and or transactivated in response to stresses such as hypoxia $[24,70]$. Not surprisingly, amongst many targets, these transcription factors induce the expression of numerous genes such as HIF-1, tissue factor, apoptosis markers, and angiogenic markers that are crucial for adapting to, or eliminating damage during, early onset of unfavorable conditions $[24,70,71]$. However, their timely activation (or suppression) is crucial for launching an appropriate response. For example, hypoxia can stimulate ETS1 expression, but it can also enhance ETS1 protein degradation via the ubiquitin proteasome system; 
and this is necessary for keeping a strict regulation on its transcriptional activity [70]. Indeed, EGR1 regulation is likely tightly controlled by its two co-repressors since they are both upregulated in wood frog livers following freezing. NAB1 is an active repressor that is tethered to the promoter region of certain genes to prevent their transcription [26]; however, NAB1 can also physically interact with EGR1 to decrease its transcriptional activity [27]. The NAB2 co-repressor also interacts with and inhibits EGR1 transcription, and is itself regulated by EGR1 in a negative feedback loop [27]. As such, it is possible that having both $\mathrm{NAB} 1$ and $\mathrm{NAB} 2$ in the nucleus is necessary to regulate the expression of certain EGR1 induced genes in a temporal manner without removing EGR1 from the nucleus.

Nuclear localization of ETS1 and EGR1 responded in a similar manner to anoxia/reoxygenation and dehydration/rehydration treatments (Figure 3.6B, 3.6C). Nuclear accumulation of ETS1 increased in response to anoxia but returned to baseline levels after reoxygenation (Figure 3.6B). Interestingly, levels of EGR1 and NAB1 remained the same, but levels of NAB2 significantly increased in response to both anoxia and reoxygenation. This suggests that perhaps at 24-hour anoxia and 4-hour reoxygenation, transcriptional activity of EGR1 is interrupted by NAB2, to prevent the untimely transcription of downstream targets. In response to dehydration and rehydration, nuclear accumulation of ETS1 increased, and while EGR1 showed similar trends, the changes were not statistically significant (Figure 3.6C). Interestingly, levels of NAB1 were decreased in response to dehydration and rehydration, while levels of NAB2 showed no change in response to dehydration but increasing after rehydration. Nonetheless, it is likely that nuclear accumulation of ETS1 and to some degree EGR1 
could occur to ensure appropriate and time-dependent expression of select downstream genes.

To assess this hypothesis, the DNA binding activity of both EGR1 and ETS1 was measured (Figure 3.7). Interestingly, despite an increase in ETS1 nuclear accumulation during freezing, the DNA binding activity of ETS1 significantly decreased after freezing and thawing (Figure 3.7A). Similar decreases in ETS1 DNA binding were seen in response to anoxia/reoxygenation and dehydration (Figure 3.7B, 3.7C). As mentioned earlier, the presence of these transcription factors in the nucleus does not necessarily suggest of active transcription at that time, specifically. It is possible that the nuclear accumulation of ETS1 was required for early hours of freezing, but because it is also required for early or late hours of thawing, it remains in the nucleus but in its inactive form to provide temporal-dependent gene expression [72]. Different posttranslational modifications or regulatory proteins could facilitate this process. Indeed, an early study showed that Sp100 physically interacts with ETS1 to enhance its transcriptional activity [73]. In addition, alternative calcium-dependent phosphorylation of four serine residues in ETS1 inhibit its DNA binding activity significantly [74]. Therefore, with respect to the evidence provided, it is possible that despite having ETS1 available in the nucleus, it may be present in its inactive form [72]. Comparably, EGR1-mediated gene expressions appeared to be regulated by its two co-repressors. While DNA binding levels remained unchanged in response to freeze/thaw (Figure 3.7A) and anoxia/reoxygenation (Figure 3.7B), EGR1 DNA binding levels increased in response to both dehydration and rehydration (Figure 3.7C). Though nuclear EGR1 levels increased in the livers of frozen and thawed wood frogs, NAB1 and NAB2 also increased, which could potentially 
prevent EGR1 DNA binding from increasing relative to the control group under the same conditions. Similarly, NAB2 levels increased in the nucleus in response to anoxia/reoxygenation to levels, which was consistent with unchanging EGR1 DNA binding levels following anoxia/reoxygenation. The decrease in levels of NAB1 in response to dehydration/rehydration treatments and $\mathrm{NAB} 2$ in response to rehydration also supports an increase in the DNA binding activity of EGR1 under the same conditions (Figure 3.6C). Therefore, EGR1, like ETS1 appears to be regulated by factors present in the nucleus to provide time-dependent gene expression activities.

The complex nature of angiogenesis requires highly regulated transcriptional responses that must occur in a spatial and a temporal manner for successful vessel formation [75]. Angiogenesis relies on signal integration from different growth factors, receptors, and adhesion molecules among other pathways. In sprouting angiogenesis, new blood vessels are formed from parent vessels in multiple steps including the proteolytic degradation of the basement membrane of capillaries, proliferation of ECs and their migration, EC tubulogenesis, fusion of the vessels and their pruning, and finally stabilization of the vascular pericytes [76]. While many transcription factors are involved, ETS1 and EGR1 have been reported to induce the transcription of several pro-angiogenic genes and are involved in wound healing and tissue repair [29-31,77-79]. The first evidence of potential angiogenic responses in wood frogs came from a study that reported an increase in fibrinogen levels in liver following early hours of freezing, dehydration, rehydration, but not in response to anoxia, possibly to facilitate wound healing [80]. Fibrinogen is cleaved into fibrin, and this is necessary for allowing EC spreading and angiogenesis. VEGF, a necessary proangiogenic protein, binds at saturating levels to both 
fibrinogen and fibrin clots to provide directionality to the site of angiogenesis [81]. Moreover, FGF2, another secreted protein involved in promoting endothelial cell proliferation has also been shown to bind to both fibrinogen and fibrin [81]. In fact, the presence of both VEGF and FGF2 function synergistically to promote angiogenesis and their attachment to fibrinogen or fibrin provides the localization and the spatial orientation information needed for maximum angiogenic responses under many conditions including wound healing $[81,82]$. Other studies report that the promoter region of VEGF contains an ETS1 binding site, whereas FGF2 expression is regulated by EGR1 [31,77]. Given our previous conclusions that ETS1 and EGR1 may be temporally regulated in response to stress in wood frogs, it is possible that their temporal regulation is involved in a timely expression of both VEGF and FGF2.

While levels of VEGF did not change in response to freezing or thawing, levels of FGF2 decreased in response to freezing but increased drastically after thawing (Figure 3.8). Since EGR1's DNA binding activity was not affected by the same treatment (Figure 3.7A), it is possible that alternative inhibitory mechanisms are involved to prevent FGF2 transcription. Indeed, while the synergistic effects of VEGF and FGF2 are needed for enhanced angiogenesis, each factor can also induce this process on their own $[81,82]$. Interestingly, the angiogenic response of wood frog livers to anoxia and reoxygenation was opposite of the response to freezing and thawing, where the levels of VEGF decreased significantly under both conditions and the levels of FGF2 remained consistent (Figure 3.9). Decreased VEGF levels correlate with decreased ETS1 DNA binding after anoxia and reoxygenation (Figure 3.7B). Therefore, it is likely that ETS1 is involved in regulating this process at that time point. While FGF2 levels remained the same in 
response to dehydration and rehydration treatments, VEGF levels only decreased in response to rehydration (Figure 3.10). The inconsistency between the DNA binding levels of ETS1 and EGR1, and protein levels of VEGF and FGF2 could be due to their temporal regulation at different durations of stress. Indeed, many of the changes required at the full onset of stress, are done either early on following exposure or are induced at later hours of recovery when energy levels are high. Alternatively, although these proteins are differentially regulated in response to each stress, their levels are not entirely depleted, and may still perform angiogenic activities albeit at lower capacity. It is possible that the expression of VEGF and FGF2 occur at either early hours of freezing, dehydration or anoxia to expand the vascular before delivering cryoprotective glucose to different organs, or later during the recovery stage in case of wound healing and dealing with injuries.

ANGPT1 and ANGPT2 are two other factors associated with angiogenesis $[83,84]$. These two secreted glycoproteins are ligands to Tie1 and Tie2, two receptor tyrosine kinases that are expressed mainly in the vascular endothelium $[84,85]$. ANGPT1 was shown to promote vascular remodeling, vascular enlargement, and stimulate EC migration, proliferation and eventual differentiation [84]. Moreover, ANGPT1 decreases the permeability of EC and stabilizes the newly formed vasculature by recruiting pericytes and smooth muscle cells to the newly formed blood vessel [86]. ANGPT2 is an antagonist to ANGPT1 that promotes vascular destabilization, enhances sprouting and branching in response to VEGF, and disrupts pericyte recruitment [87]. As such, ANGPT2-induced destabilization of the vasculature promotes angiogenesis in response to VEGF, but in the absence of VEGF, ANGPT2 causes vessel regression $[85,88]$. Their 
activities must be carefully regulated to ensure appropriate angiogenesis, as one promotes the onset of the process while the other stabilizes the newly formed vessels.

Interestingly, ANGPT1 expression has been shown to enhance the expression and activation of EGR1, which in turn induced pro-angiogenic factors such as FGF2, interleukin 8, VEGFR1 and the platelet derived growth factor B [30]. In addition, EGR1 was also shown to promote the expression of several matrix metalloproteinases, which are necessary for angiogenesis [46]. ETS1 was shown to bind to the promoter region of ANGPT2, and induce its expression [83]. Moreover, other members of the ETS transcription family were shown to regulate the expression of both Tie1 and Tie2 during angiogenesis [29]. The results of this study show that freezing increased ANGPT1 levels, whereas both freezing and thawing increased ANGPT2 levels (Figure 3.8). Tie1 and VEGFR1 levels only increased in response to thawing, whereas Tie2 levels increased under both treatment conditions (Figure 3.8). The presence of both ANGPT1 and ANGPT2 and their receptors presents a tug-of-war scenario between promoting angiogenesis and restricting it. Indeed, ANGPT2 can only promote angiogenesis in presence of VEGF $[85,88]$. Moreover, while VEGF binds to VEGFR1 with higher affinity, the kinase activity of this receptor is relatively weaker than that of VEGFVEGFR2 [89]. Given the high affinity of VEGF for VEGFR1, VEGFR1 can act as a suppressor of angiogenesis by sequestering the available VEGF in a spatial manner until its needed $[89,90]$. Therefore, even though VEGF levels did not change in response to treatment, it is possible that the basal amount present is necessary to promote low levels of angiogenic responses to fulfill wood frog requirement for blood vessel formation or 
wound healing when needed and that VEGFR1 provides the required VEGF sequestration.

In response to dehydration and rehydration, levels of ANGPT1, ANGPT2, Tie1 and Tie2 increased under both conditions. Like freezing, the presence of both proangiogenic and antiangiogenic factors provides the cell with a choice of inducing or repressing angiogenesis whenever needed in response to dehydration/rehydration treatments. Indeed, formation of ice crystals during freezing and significant reduction in cell volume during dehydration could result in capillary damage or tissue injury; therefore, having proangiogenic factors at hand could result in new vessel formation or capillary and tissue repair when needed. Responses to anoxia/reoxygenation treatments were somewhat different. While ANGPT1 and VEGFR1 levels remain the same in response to anoxia and reoxygenation (Figure 3.9), levels of ANGPT2 show a decrease in response to reoxygenation, whereas levels of Tie1 and Tie2 increase in response to both conditions (Figure 3.9). These results suggest that perhaps angiogenesis or tissue repair is occurring at minimal level in response to anoxia and reoxygenation. This is because despite not having an increase in growth factors (VEGF, FGF2, ANGPT2), levels of Tie 1 and Tie2 increased; suggesting that perhaps the low levels of secreted growth factors available is binding to and activating downstream angiogenic signals. While plausible, further investigation is warranted.

VE-cad is an adhesion molecule specific to EC and interacts homophilically with neighboring cells to provide strength and stability to the endothelium. Binding of VEGF to VEGFR2 activates its tyrosine kinase domain, and through a sequence of events, results in the phosphorylation of VE-cad [91]. This phosphorylation event results in 
internalization of VE-cad and the destabilization of the endothelium [92]. As such, levels of VE-cad are reduced in response to angiogenesis to destabilize the endothelium for subsequent growth. The proximal promoter region of VE-cad harbors an ETS-binding site, and a previous study showed that ETS1 carries the transactivation of this promoter [93]. Results in this study show that total protein levels of VE-cad increased in response to freezing, thawing (Figure 3.8), and dehydration (Figure 3.10), but was reduced in response to reoxygenation (Figure 3.9). The increase in VE-cad correlates with the increase in ETS1 nuclear localization seen in this study in response to freeze/thaw and dehydration/rehydration, but not with ETS1 DNA binding activity. Oppositely, the reduction in DNA binding activity of ETS in response to reoxygenation correlates with protein levels of VE-cad. The discrepancy between the binding can be explained by two reasons. First, as mentioned earlier, angiogenesis is a complex process that occurs in a spatiotemporal manner with multiple activators and repressors at play [75]. Angiogenic responses in wood frogs might occur in response to early periods of freezing or dehydration to facilitate the distribution of cryoprotective glucose to different organs and upon respective recoveries to heal injured tissue or capillaries. As such it is possible that ETS1 activation in the early hours of freezing resulted in the induction of VE-cad and other angiogenic factors in anticipation and preparation for future events but is interrupted once enough factors are transcribed. With respect to reoxygenation, a decrease in both VE-cad could indicate pro-angiogenic responses, but that this response might occur at basal level depending on the availability of other growth factors. A second reason for the discrepancy between ETS1 binding and downstream targets could be regulated by posttranslational modifications and changes in subcellular localization. For 
example, given the limited energy available, it is possible that the low levels of proangiogenic factors available are post-translationally modified to maintain their activity at desired level. Indeed, VE-cad molecules are destabilized in response to phosphorylation in response to VEGFR2 [91]. Given that VEGFR2 proteins were below the detection limit in this study, it is possible that absence of this phosphorylation event is another mechanism to keep angiogenic activities at low level. In fact, high levels of VE-cad in response to freezing and anoxia together with absence of VEGFR2 is suggestive of a highly stable endothelium, and attenuated angiogenesis.

In conclusion, this study has demonstrated to that the HMGB1/AGE/RAGE axis might be involved in regulating angiogenesis by inducing or transactivating ETS1 and EGR1, two transcription factors that are involved in angiogenesis. Current results also show that both ETS1 and EGR1 appear to be induced in early hours of treatment (especially in response to freezing and dehydration) to upregulate pro- and antiangiogenic factors in preparation for potential vessel expansion or wound healing. The presence of both activators and repressors of angiogenesis enable the cell to suppress angiogenic responses when they are not required, but to induce this process when needed.

\section{Acknowledgement}

We thank J.M. Storey for editorial review of this manuscript. This work was supported by a Discovery Grant from the Natural Sciences and Engineering Research Council of Canada (Grant \#6793). K.B.S. holds the Canadian Research Chair in Molecular Physiology and R.A. holds an Ontario Graduate Scholarship. 


\subsection{References- Chapter 3}

[1] K.B. Storey, J.M. Storey, Molecular physiology of freeze tolerance in vertebrates, Physiol. Rev. 97 (2017) 623-665. https://doi.org/10.1152/physrev.00016.2016.

[2] K.B. Storey, J.M. Storey, Freeze tolerance in animals, Physiol. Rev. 68 (1988) $27-$ 84. https://doi.org/10.1152/physrev.1988.68.1.27.

[3] T.A. Churchill, K.B. Storey, Dehydration tolerance in wood frogs: A new perspective on development of amphibian freeze tolerance, Am. J. Physiol. Integr. Comp. Physiol. 265 (1993) R1324-R1332.

https://doi.org/10.1152/ajpregu.1993.265.6.R1324.

[4] C.P. Holden, K.B. Storey, Second messenger and cAMP-dependent protein kinase responses to dehydration and anoxia stresses in frogs., J. Comp. Physiol. B. 167 (1997) 305-12. https://doi.org/10.1007/s003600050078.

[5] C.W. Wu, K.K. Biggar, J. Zhang, S.N. Tessier, F. Pifferi, M. Perret, K.B. Storey, Induction of antioxidant and heat shock protein responses during torpor in the Gray Mouse Lemur, Microcebus murinus, Genomics, Proteomics Bioinforma. 13 (2015) 19-126. https://doi.org/10.1016/j.gpb.2015.03.004.

[6] D.R. Joanisse, K.B. Storey, Oxidative damage and antioxidants in Rana sylvatica, the freeze-tolerant wood frog., Am. J. Physiol. 271 (1996) R545-53. https://doi.org/10.1152/ajpregu.1996.271.3.R545.

[7] V.E.M. Gerber, S. Wijenayake, K.B. Storey, Anti-apoptotic response during anoxia and recovery in a freeze-tolerant wood frog (Rana sylvatica)., PeerJ. 4 (2016) e1834. https://doi.org/10.7717/peerj.1834.

[8] K.B. Storey, J.M. Storey, Biochemical adaption for freezing tolerance in the wood frog, Rana sylvatica, J. Comp. Physiol. B. 155 (1984) 29-36. https://doi.org/10.1007/BF00688788.

[9] J.W. Baynes, N.G. Watkins, C.I. Fisher, C.J. Hull, J.S. Patrick, M.U. Ahmed, J.A. Dunn, S.R. Thorpe, The Amadori product on protein: Structure and reactions., Prog. Clin. Biol. Res. 304 (1989) 43-67. http://www.ncbi.nlm.nih.gov/pubmed/2675036.

[10] T.J. Lyons, A.J. Jenkins, Glycation, oxidation, and lipoxidation in the development of the complications of diabetes: A carbonyl stress hypothesis., Diabetes Rev. (Alexandria, Va.). 5 (1997) 365-391.

[11] K. Ikeda, T. Higashi, H. Sano, Y. Jinnouchi, M. Yoshida, T. Araki, S. Ueda, S. Horiuchi, $\mathrm{N} \varepsilon$-(carboxymethyl)lysine protein adduct is a major immunological epitope in proteins modified with advanced glycation end products of the Maillard reaction $\dagger$, Biochemistry. 35 (1996) 8075-8083. https://doi.org/10.1021/bi9530550.

[12] T. Miyata, K. Kurokawa, C. Van Ypersele De Strihou, Advanced glycation and lipoxidation end products: Role of reactive carbonyl compounds generated during 
carbohydrate and lipid metabolism., J. Am. Soc. Nephrol. 11 (2000) 1744-52. http://www.ncbi.nlm.nih.gov/pubmed/10966500.

[13] A.W. Stitt, J.E. Moore, J.A. Sharkey, G. Murphy, D.A.C. Simpson, R. Bucala, H. Vlassara, D.B. Archer, Advanced glycation end products in vitreous: Structural and functional implications for diabetic vitreopathy., Invest. Ophthalmol. Vis. Sci. 39 (1998) 2517-23. http://www.ncbi.nlm.nih.gov/pubmed/9856760.

[14] J.A. MacDonald, T. Degenhardt, J.W. Baynes, K.B. Storey, Glycation of wood frog (Rana sylvatica) hemoglobin and blood proteins: In vivo and in vitro studies, Cryobiology. 59 (2009) 223-225. https://doi.org/10.1016/j.cryobiol.2009.06.008.

[15] A. Bierhaus, P.M. Humpert, M. Morcos, T. Wendt, T. Chavakis, B. Arnold, D.M. Stern, P.P. Nawroth, Understanding RAGE, the receptor for advanced glycation end products, J. Mol. Med. 83 (2005) 876-886. https://doi.org/10.1007/s00109005-0688-7.

[16] H. Yang, H. Wang, S.S. Chavan, U. Andersson, High mobility group box protein 1 (HMGB1): The prototypical endogenous danger molecule, Mol. Med. 21 (2015) S6-S12. https://doi.org/10.2119/molmed.2015.00087.

[17] B. Lu, D.J. Antoine, K. Kwan, P. Lundback, H. Wahamaa, H. Schierbeck, M. Robinson, M.A.D. Van Zoelen, H. Yang, J. Li, H. Erlandsson-Harris, S.S. Chavan, H. Wang, U. Andersson, K.J. Tracey, JAK/STAT1 signaling promotes HMGB1 hyperacetylation and nuclear translocation, Proc. Natl. Acad. Sci. 111 (2014) 3068-3073. https://doi.org/10.1073/pnas.1316925111.

[18] R. Kang, K.M. Livesey, H.J. Zeh, M.T. Lotze, D. Tang, HMGB1: A novel Beclin 1-binding protein active in autophagy, Autophagy. 6 (2010) 1209-1211. https://doi.org/10.4161/auto.6.8.13651.

[19] S. Gardella, C. Andrei, D. Ferrera, L. V. Lotti, M.R. Torrisi, M.E. Bianchi, A. Rubartelli, The nuclear protein HMGB1 is secreted by monocytes via a nonclassical, vesicle-mediated secretory pathway, EMBO Rep. 3 (2002) 995-1001. https://doi.org/10.1093/embo-reports/kvf198.

[20] L.G. Bucciarelli, M. Kaneko, R. Ananthakrishnan, E. Harja, L.K. Lee, Y.C. Hwang, S. Lerner, S. Bakr, Q. Li, Y. Lu, F. Song, W. Qu, T. Gomez, S.Z. Yu, F.Y. Shi, A.M. Schmidt, R. Ramasamy, Receptor for advanced-glycation end products: Key modulator of myocardial ischemic injury, Circulation. 113 (2006) 1226-1234. https://doi.org/10.1161/CIRCULATIONAHA.105.575993.

[21] K.B. Storey, Strategies for exploration of freeze responsive gene expression: Advances in vertebrate freeze tolerance, Cryobiology. 48 (2004) 134-145. https://doi.org/10.1016/j.cryobiol.2003.10.008.

[22] Y. Xu, F. Toure, W. Qu, L. Lin, F. Song, X. Shen, R. Rosario, J. Garcia, A.M. Schmidt, S.F. Yan, Advanced glycation end product (AGE)-receptor for age (RAGE) signaling and up-regulation of Egr-1 in hypoxic macrophages, J. Biol. Chem. 285 (2010) 23233-23240. https://doi.org/10.1074/jbc.M110.117457. 
[23] J.S. Chang, T. Wendt, W. Qu, L. Kong, Y.S. Zou, A.M. Schmidt, S.F. Yan, Oxygen deprivation triggers upregulation of early growth response-1 by the receptor for advanced glycation end products, Circ. Res. 102 (2008) 905-913. https://doi.org/10.1161/CIRCRESAHA.107.165308.

[24] S.F. Yan, J. Lu, Y.S. Zou, J. Soh-Won, D.M. Cohen, P.M. Buttrick, D.R. Cooper, S.F. Steinberg, N. Mackman, D.J. Pinsky, D.M. Stern, Hypoxia-associated induction of early growth response-1 gene expression, J. Biol. Chem. 274 (1999) 15030-15040. https://doi.org/10.1074/jbc.274.21.15030.

[25] J.-I. Pagel, E. Deindl, Early growth response 1-a transcription factor in the crossfire of signal transduction cascades., Indian J. Biochem. Biophys. 48 (2011) 226-35. http://www.ncbi.nlm.nih.gov/pubmed/22053691.

[26] A.H. Swirnoff, E.D. Apel, J. Svaren, B.R. Sevetson, D.B. Zimonjic, N.C. Popescu, J. Milbrandt, Nab1, a corepressor of NGFI-A (Egr-1), contains an active transcriptional repression domain, Mol. Cell. Biol. 18 (1998) 512-524. https://doi.org/10.1128/MCB.18.1.512.

[27] J. Kumbrink, M. Gerlinger, J.P. Johnson, Egr-1 induces the expression of its corepressor Nab2 by activation of the Nab2 promoter thereby establishing a negative feedback loop, J. Biol. Chem. 280 (2005) 42785-42793. https://doi.org/10.1074/jbc.M511079200.

[28] W.K. Wang, Q.H. Lu, J.N. Zhang, B. Wang, X.J. Liu, F.S. An, W.D. Qin, X.Y. Chen, W.Q. Dong, C. Zhang, Y. Zhang, M.X. Zhang, HMGB1 mediates hyperglycaemia-induced cardiomyocyte apoptosis via ERK/Ets-1 signalling pathway, J. Cell. Mol. Med. 18 (2014) 2311-2320.

https://doi.org/10.1111/jcmm.12399.

[29] A.M. Randi, A. Sperone, N.H. Dryden, G.M. Birdsey, Regulation of angiogenesis by ETS transcription factors., Biochem. Soc. Trans. 37 (2009) 1248-53. https://doi.org/10.1042/BST0371248.

[30] N.A. Abdel-Malak, M. Mofarrahi, D. Mayaki, L.M. Khachigian, S.N.A. Hussain, Early growth response-1 regulates angiopoietin-1-induced endothelial cell proliferation, migration, and differentiation, Arterioscler. Thromb. Vasc. Biol. 29 (2009) 209-216. https://doi.org/10.1161/ATVBAHA.108.181073.

[31] R.G. Fahmy, C.R. Dass, L.-Q. Sun, C.N. Chesterman, L.M. Khachigian, Transcription factor Egr-1 supports FGF-dependent angiogenesis during neovascularization and tumor growth, Nat. Med. 9 (2003) 1026-1032. https://doi.org/10.1038/nm905.

[32] T. Tonini, F. Rossi, P.P. Claudio, Molecular basis of angiogenesis and cancer, Oncogene. 22 (2003) 6549-6556. https://doi.org/10.1038/sj.onc.1206816.

[33] T. Johnson, L. Zhao, G. Manuel, H. Taylor, D. Liu, Approaches to therapeutic angiogenesis for ischemic heart disease, J. Mol. Med. 97 (2019) 141-151. https://doi.org/10.1007/s00109-018-1729-3. 
[34] P. Kumar, S. Kumar, E.P. Udupa, U. Kumar, P. Rao, T. Honnegowda, Role of angiogenesis and angiogenic factors in acute and chronic wound healing, Plast. Aesthetic Res. 2 (2015) 243. https://doi.org/10.4103/2347-9264.165438.

[35] M.G. Tonnesen, X. Feng, R.A.F. Clark, Angiogenesis in wound healing, J. Investig. Dermatology Symp. Proc. 5 (2000) 40-46. https://doi.org/10.1046/j.1087-0024.2000.00014.x.

[36] R. Al-attar, Y. Zhang, K.B. Storey, Osmolyte regulation by TonEBP/NFAT5 during anoxia-recovery and dehydration-rehydration stresses in the freeze-tolerant wood frog (Rana sylvatica), PeerJ. 5 (2017) e2797. https://doi.org/10.7717/peerj.2797.

[37] R. Al-Attar, S. Wijenayake, K.B. Storey, Metabolic reorganization in winter: Regulation of pyruvate dehydrogenase (PDH) during long-term freezing and anoxia., Cryobiology. 86 (2019) 10-18. https://doi.org/10.1016/j.cryobiol.2019.01.006.

[38] J.P. Costanzo, R.E. Lee, Avoidance and tolerance of freezing in ectothermic vertebrates, J. Exp. Biol. 216 (2013) 1961-1967. https://doi.org/10.1242/jeb.070268.

[39] R. Al-Attar, K.B. Storey, Effects of anoxic exposure on the nuclear factor of activated T cell (NFAT) transcription factors in the stress-tolerant wood frog., Cell Biochem. Funct. 36 (2018) 420-430. https://doi.org/10.1002/cbf.3362.

[40] S.G. English, H. Hadj-Moussa, K.B. Storey, MicroRNAs regulate survival in oxygen-deprived environments, J. Exp. Biol. 221 (2018) jeb190579. https://doi.org/10.1242/jeb.190579.

[41] M.P. Hoyeck, H. Hadj-Moussa, K.B. Storey, The role of MEF2 transcription factors in dehydration and anoxia survival in Rana sylvatica skeletal muscle, PeerJ. 5 (2017) e4014. https://doi.org/10.7717/peerj.4014.

[42] S.A. Bustin, V. Benes, J.A. Garson, J. Hellemans, J. Huggett, M. Kubista, R. Mueller, T. Nolan, M.W. Pfaffl, G.L. Shipley, J. Vandesompele, C.T. Wittwer, The MIQE guidelines: Minimum information for publication of quantitative realtime PCR experiments, Clin. Chem. 55 (2009) 611-622. https://doi.org/10.1373/clinchem.2008.112797.

[43] J. Zhang, K.B. Storey, Akt signaling and freezing survival in the wood frog, Rana sylvatica, Biochim. Biophys. Acta. 1830 (2013) 4828-37. https://doi.org/10.1016/j.bbagen.2013.06.020.

[44] S.L. Eaton, S.L. Roche, M. Llavero Hurtado, K.J. Oldknow, C. Farquharson, T.H. Gillingwater, T.M. Wishart, Total protein analysis as a reliable loading control for quantitative fluorescent western blotting, PLoS One. 8 (2013) e72457. https://doi.org/10.1371/journal.pone.0072457.

[45] J. Zhang, K.B. Storey, RBioplot: An easy-to-use R pipeline for automated statistical analysis and data visualization in molecular biology and biochemistry, 
PeerJ. 4 (2016) e2436. https://doi.org/10.7717/peerj.2436.

[46] T.L. Haas, D. Stitelman, S.J. Davis, S.S. Apte, J.A. Madri, Egr-1 mediates extracellular matrix-driven transcription of membrane type 1 matrix metalloproteinase in endothelium, J. Biol. Chem. 274 (1999) 22679-22685. https://doi.org/10.1074/jbc.274.32.22679.

[47] T. Bonaldi, F. Talamo, P. Scaffidi, D. Ferrera, A. Porto, A. Bachi, A. Rubartelli, A. Agresti, M.E. Bianchi, Monocytic cells hyperacetylate chromatin protein HMGB1 to redirect it towards secretion, EMBO J. 22 (2003) 5551-5560. https://doi.org/10.1093/emboj/cdg516.

[48] X. Zhu, J.S. Messer, Y. Wang, F. Lin, C.M. Cham, J. Chang, T.R. Billiar, M.T. Lotze, D.L. Boone, E.B. Chang, Cytosolic HMGB1 controls the cellular autophagy/apoptosis checkpoint during inflammation, J. Clin. Invest. 125 (2015) 1098-1110. https://doi.org/10.1172/JCI76344.

[49] J.S. Park, F. Gamboni-Robertson, Q. He, D. Svetkauskaite, J.-Y. Kim, D. Strassheim, J.-W. Sohn, S. Yamada, I. Maruyama, A. Banerjee, A. Ishizaka, E. Abraham, High mobility group box 1 protein interacts with multiple Toll-like receptors., Am. J. Physiol. Cell Physiol. 290 (2006) C917-24. https://doi.org/10.1152/ajpcell.00401.2005.

[50] A. Petrović, D. Bogojević, A. Korać, I. Golić, S. Jovanović-Stojanov, V. Martinović, S. Ivanović-Matić, J. Stevanović, G. Poznanović, I. Grigorov, Oxidative stress-dependent contribution of HMGB1 to the interplay between apoptosis and autophagy in diabetic rat liver, J. Physiol. Biochem. 73 (2017) 511521. https://doi.org/10.1007/s13105-017-0574-0.

[51] H. Wu, Z. Chen, J. Xie, L.-N. Kang, L. Wang, B. Xu, High mobility group box-1: A missing link between diabetes and its complications, Mediators Inflamm. 2016 (2016) 1-11. https://doi.org/10.1155/2016/3896147.

[52] S. Yang, L. Xu, T. Yang, F. Wang, High-mobility group box-1 and its role in angiogenesis, J. Leukoc. Biol. 95 (2014) 563-574.

https://doi.org/10.1189/jlb.0713412.

[53] M. Lamkanfi, A. Sarkar, L. Vande Walle, A.C. Vitari, A.O. Amer, M.D. Wewers, K.J. Tracey, T.-D. Kanneganti, V.M. Dixit, Inflammasome-dependent release of the alarmin HMGB1 in endotoxemia, J. Immunol. 185 (2010) 4385-4392. https://doi.org/10.4049/jimmunol.1000803.

[54] S.S. Lange, K.M. Vasquez, HMGB1: The jack-of-all-trades protein is a master DNA repair mechanic, Mol. Carcinog. 48 (2009) 571-580. https://doi.org/10.1002/mc.20544.

[55] J. Evankovich, S.W. Cho, R. Zhang, J. Cardinal, R. Dhupar, L. Zhang, J.R. Klune, J. Zlotnicki, T. Billiar, A. Tsung, High mobility group box 1 release from hepatocytes during ischemia and reperfusion injury is mediated by decreased histone deacetylase activity., J. Biol. Chem. 285 (2010) 39888-97.

https://doi.org/10.1074/jbc.M110.128348. 
[56] Y. Tang, X. Zhao, D. Antoine, X. Xiao, H. Wang, U. Andersson, T.R. Billiar, K.J. Tracey, B. Lu, Regulation of Posttranslational Modifications of HMGB1 During Immune Responses, Antioxid. Redox Signal. 24 (2016) 620-634. https://doi.org/10.1089/ars.2015.6409.

[57] C. Janko, M. Filipović, L.E. Munoz, C. Schorn, G. Schett, I. Ivanović-Burmazović, M. Herrmann, Redox modulation of HMGB1-related signaling, Antioxid. Redox Signal. 20 (2014) 1075-1085. https://doi.org/10.1089/ars.2013.5179.

[58] D. Tang, M.T. Loze, H.J. Zeh, III, R. Kang, The redox protein HMGB1 regulates cell death and survival in cancer treatment, Autophagy. 6 (2010) 1181-1183. https://doi.org/10.4161/auto.6.8.13367.

[59] D. Yao, M. Brownlee, Hyperglycemia-induced reactive oxygen species increase expression of the receptor for advanced glycation end products (RAGE) and RAGE ligands, Diabetes. 59 (2010) 249-255. https://doi.org/db09-0801 [pii];10.2337/db09-0801 [doi].

[60] M. Fournet, F. Bonté, A. Desmoulière, Glycation damage: A possible hub for major pathophysiological disorders and aging, Aging Dis. 9 (2018) 880. https://doi.org/10.14336/AD.2017.1121.

[61] E.M. Ellis, Reactive carbonyls and oxidative stress: Potential for therapeutic intervention, Pharmacol. Ther. 115 (2007) 13-24. https://doi.org/10.1016/j.pharmthera.2007.03.015.

[62] A. Gupta, K.B. Storey, Regulation of antioxidant systems in response to anoxia and reoxygenation in Rana sylvatica, Comp. Biochem. Physiol. Part B Biochem. Mol. Biol. 243-244 (2020) 110436. https://doi.org/10.1016/j.cbpb.2020.110436.

[63] C.-W. Wu, S.N. Tessier, K.B. Storey, Stress-induced antioxidant defense and protein chaperone response in the freeze-tolerant wood frog Rana sylvatica, Cell Stress Chaperones. 23 (2018) 1205-1217. https://doi.org/10.1007/s12192-0180926-x.

[64] K.K. Biggar, K.B. Storey, The emerging roles of microRNAs in the molecular responses of metabolic rate depression, J. Mol. Cell Biol. 3 (2011) 167-175. https://doi.org/10.1093/jmcb/mjq045.

[65] W. Wei, Z. Hu, H. Fu, Y. Tie, H. Zhang, Y. Wu, X. ZHeng, MicroRNA-1 and microRNA-499 downregulate the expression of the ets1 proto-oncogene in HepG2 cells, Oncol. Rep. 28 (2012) 701-706. https://doi.org/10.3892/or.2012.1850.

[66] S.C. Greenway, K.B. Storey, Activation of mitogen-activated protein kinases during natural freezing and thawing in the wood frog, Mol. Cell. Biochem. 209 (2000) 29-37. https://doi.org/10.1023/a:1007077522680.

[67] S.C. Greenway, K.B. Storey, Discordant responses of mitogen-activated protein kinases to anoxia and freezing exposures in hatchling turtles, J. Comp. Physiol. B Biochem. Syst. Environ. Physiol. 169 (1999) 521-527.

https://doi.org/10.1007/s003600050251. 
[68] A.I. Malik, K.B. Storey, Activation of extracellular signal-regulated kinases during dehydration in the African clawed frog, Xenopus laevis., J. Exp. Biol. 212 (2009) 2595-603. https://doi.org/10.1242/jeb.030627.

[69] R. Al-Attar, K.B. Storey, Suspended in time: Molecular responses to hibernation also promote longevity., Exp. Gerontol. 134 (2020) 110889. https://doi.org/10.1016/j.exger.2020.110889.

[70] N. Qiao, C. Xu, Y.-X. Zhu, Y. Cao, D.-C. Liu, X. Han, Ets-1 as an early response gene against hypoxia-induced apoptosis in pancreatic $\beta$-cells., Cell Death Dis. 6 (2015) e1650. https://doi.org/10.1038/cddis.2015.8.

[71] T. Oikawa, T. Yamada, Molecular biology of the Ets family of transcription factors, Gene. 303 (2003) 11-34. https://doi.org/10.1016/S0378-1119(02)01156-3.

[72] J.S. Yordy, R.C. Muise-Helmericks, Signal transduction and the Ets family of transcription factors, Oncogene. 19 (2000) 6503-6513. https://doi.org/10.1038/sj.onc.1204036.

[73] C. Wasylyk, S.E. Schlumberger, P. Criqui-Filipe, B. Wasylyk, Sp100 interacts with ETS-1 and stimulates its transcriptional activity, Mol. Cell. Biol. 22 (2002) 2687-2702. https://doi.org/10.1128/MCB.22.8.2687-2702.2002.

[74] D.O. Cowley, B.J. Graves, Phosphorylation represses Ets-1 DNA binding by reinforcing autoinhibition., Genes Dev. 14 (2000) 366-76. https://doi.org/10.1101/gad.14.3.366.

[75] K. Bentley, S. Chakravartula, The temporal basis of angiogenesis, Philos. Trans. R. Soc. B Biol. Sci. 372 (2017) 20150522. https://doi.org/10.1098/rstb.2015.0522.

[76] D. Ribatti, E. Crivellato, "Sprouting angiogenesis", a reappraisal, Dev. Biol. 372 (2012) 157-165. https://doi.org/10.1016/j.ydbio.2012.09.018.

[77] N. Hashiya, N. Jo, M. Aoki, K. Matsumoto, T. Nakamura, Y. Sato, N. Ogata, T. Ogihara, Y. Kaneda, R. Morishita, In vivo evidence of angiogenesis induced by transcription factor Ets-1[1] N. Hashiya, N. Jo, M. Aoki, K. Matsumoto, T. Nakamura, Y. Sato, N. Ogata, T. Ogihara, Y. Kaneda, R. Morishita, In vivo evidence of angiogenesis induced by transcription facto, Circulation. 109 (2004) 3035-3041. https://doi.org/10.1161/01.CIR.0000130643.41587.DB.

[78] W.K. Kim, Y. Kwon, M. Park, S. Yun, J.-Y. Kwon, H. Kim, Identification of specifically activated angiogenic molecules in HMGB-1-induced angiogenesis, BMB Rep. 50 (2017) 590-595. https://doi.org/10.5483/BMBRep.2017.50.11.129.

[79] M. Bryant, G.M. Drew, P. Houston, P. Hissey, C.J. Campbell, M. Braddock, Tissue repair with a therapeutic transcription factor., Hum. Gene Ther. 11 (2000) 2143-2158. https://doi.org/10.1089/104303400750001444.

[80] Q. Cai, K.B. Storey, Freezing-induced genes in wood frog (Rana sylvatica): fibrinogen upregulation by freezing and dehydration, Am. J. Physiol. Integr. Comp. Physiol. 272 (1997) R1480-R1492. https://doi.org/10.1152/ajpregu.1997.272.5.R1480. 
[81] A. Sahni, C.W. Francis, Vascular endothelial growth factor binds to fibrinogen and fibrin and stimulates endothelial cell proliferation, Blood. 96 (2000) 3772-3778. https://doi.org/10.1182/blood.V96.12.3772.h8003772_3772_3778.

[82] M.S. Pepper, S.J. Mandriota, M. Jeltsch, V. Kumar, K. Alitalo, Vascular endothelial growth factor (VEGF)-C synergizes with basic fibroblast growth factor and VEGF in the induction of angiogenesis in vitro and alters endothelial cell extracellular proteolytic activity, J. Cell. Physiol. 177 (1998) 439-452. https://doi.org/10.1002/(SICI)1097-4652(199812)177:3<439::AIDJCP7>3.0.CO;2-2.

[83] Y. Hasegawa, M. Abe, T. Yamazaki, O. Niizeki, K. Shiiba, I. Sasaki, Y. Sato, Transcriptional regulation of human angiopoietin-2 by transcription factor Ets-1, Biochem. Biophys. Res. Commun. 316 (2004) 52-58. https://doi.org/10.1016/j.bbrc.2004.02.019.

[84] N.P.J. Brindle, P. Saharinen, K. Alitalo, Signaling and functions of angiopoietin-1 in vascular protection., Circ. Res. 98 (2006) 1014-23. https://doi.org/10.1161/01.RES.0000218275.54089.12.

[85] T. Asahara, D. Chen, T. Takahashi, K. Fujikawa, M. Kearney, M. Magner, G.D. Yancopoulos, J.M. Isner, Tie2 Receptor Ligands, Angiopoietin-1 and Angiopoietin-2, Modulate VEGF-Induced Postnatal Neovascularization, Circ. Res. 83 (1998) 233-240. https://doi.org/10.1161/01.RES.83.3.233.

[86] S. Davis, T.H. Aldrich, P.F. Jones, A. Acheson, D.L. Compton, V. Jain, T.E. Ryan, J. Bruno, C. Radziejewski, P.C. Maisonpierre, G.D. Yancopoulos, Isolation of angiopoietin-1, a ligand for the TIE2 receptor, by secretion-trap expression cloning, Cell. 87 (1996) 1161-1169. https://doi.org/10.1016/S00928674(00)81812-7.

[87] E. Fagiani, P. Lorentz, L. Kopfstein, G. Christofori, Angiopoietin-1 and -2 exert antagonistic functions in tumor angiogenesis, yet both induce lymphangiogenesis, Cancer Res. 71 (2011) 5717-5727. https://doi.org/10.1158/0008-5472.CAN-104635.

[88] J. Holash, Vessel Cooption, Regression, and Growth in Tumors Mediated by Angiopoietins and VEGF, Science (80-. ). 284 (1999) 1994-1998. https://doi.org/10.1126/science.284.5422.1994.

[89] M. Shibuya, Vascular endothelial growth factor receptor-1 (VEGFR-1/Flt-1): a dual regulator for angiogenesis, Angiogenesis. 9 (2006) 225-230. https://doi.org/10.1007/s10456-006-9055-8.

[90] S. Hiratsuka, K. Nakao, K. Nakamura, M. Katsuki, Y. Maru, M. Shibuya, Membrane fixation of vascular endothelial growth factor receptor 1 ligand-binding domain is important for sasculogenesis and angiogenesis in Mice, Mol. Cell. Biol. 25 (2005) 346-354. https://doi.org/10.1128/MCB.25.1.346-354.2005.

[91] N. Lambeng, Y. Wallez, C. Rampon, F. Cand, G. Christé, D. Gulino-Debrac, I. Vilgrain, P. Huber, Vascular endothelial-cadherin tyrosine phosphorylation in 
angiogenic and quiescent adult tissues, Circ. Res. 96 (2005) 384-391.

https://doi.org/10.1161/01.RES.0000156652.99586.9f.

[92] K. Xiao, J. Garner, K.M. Buckley, P.A. Vincent, C.M. Chiasson, E. Dejana, V. Faundez, A.P. Kowalczyk, p120-catenin regulates clathrin-dependent endocytosis of Ve-cadherin, Mol. Biol. Cell. 16 (2005) 5141-5151.

https://doi.org/10.1091/mbc.e05-05-0440.

[93] E. Lelièvre, V. Mattot, P. Huber, B. Vandenbunder, F. Soncin, ETS1 lowers capillary endothelial cell density at confluence and induces the expression of VEcadherin, Oncogene. 19 (2000) 2438-2446.

https://doi.org/10.1038/sj.onc.1203563. 


\section{Figures}

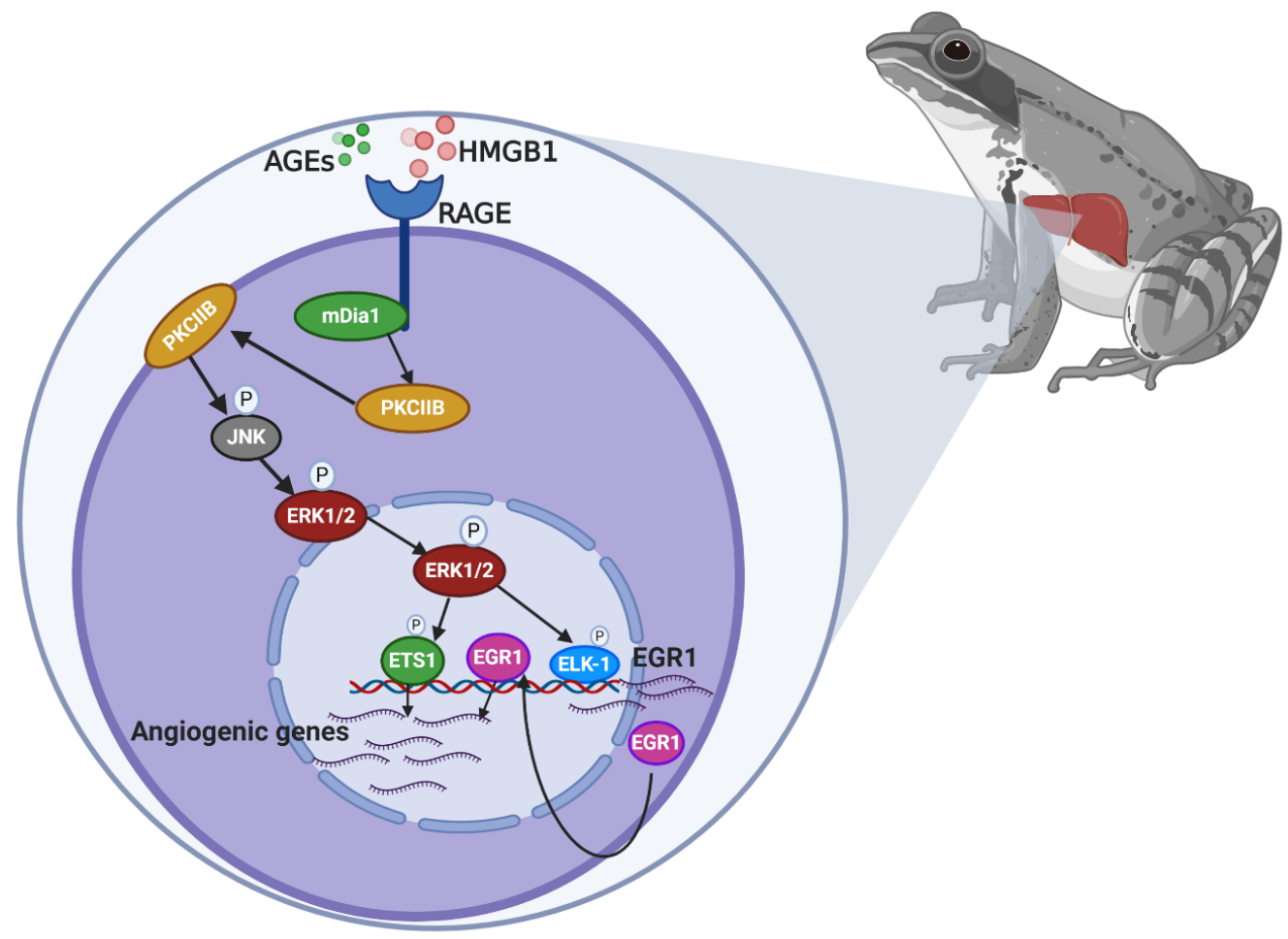

Figure 3.1: Schematic diagram of HMGB1/AGE/RAGE-induced transcriptional responses leading to angiogenic gene transcription. Upon binding of AGEs or HMGB1 to the extracellular domain of RAGE, the cytoplasmic domain of this receptor interacts with and activates mDial, which then promotes the translocation of PKC $\beta I$ to the cell membrane. This translocation allows the phosphorylation of JNK and subsequently ERK1/2, leading to the phosphorylation and activation of the transcription factors ETS1 and ELK-1. ELK-1 transcribed another transcription factor called EGR1, and upon its translation, EGR1 returns to the nucleus and together with ETS1 promote the expression of a gene network that regulates angiogenesis. Illustration was created by BioRender (https://biorender.com/). 

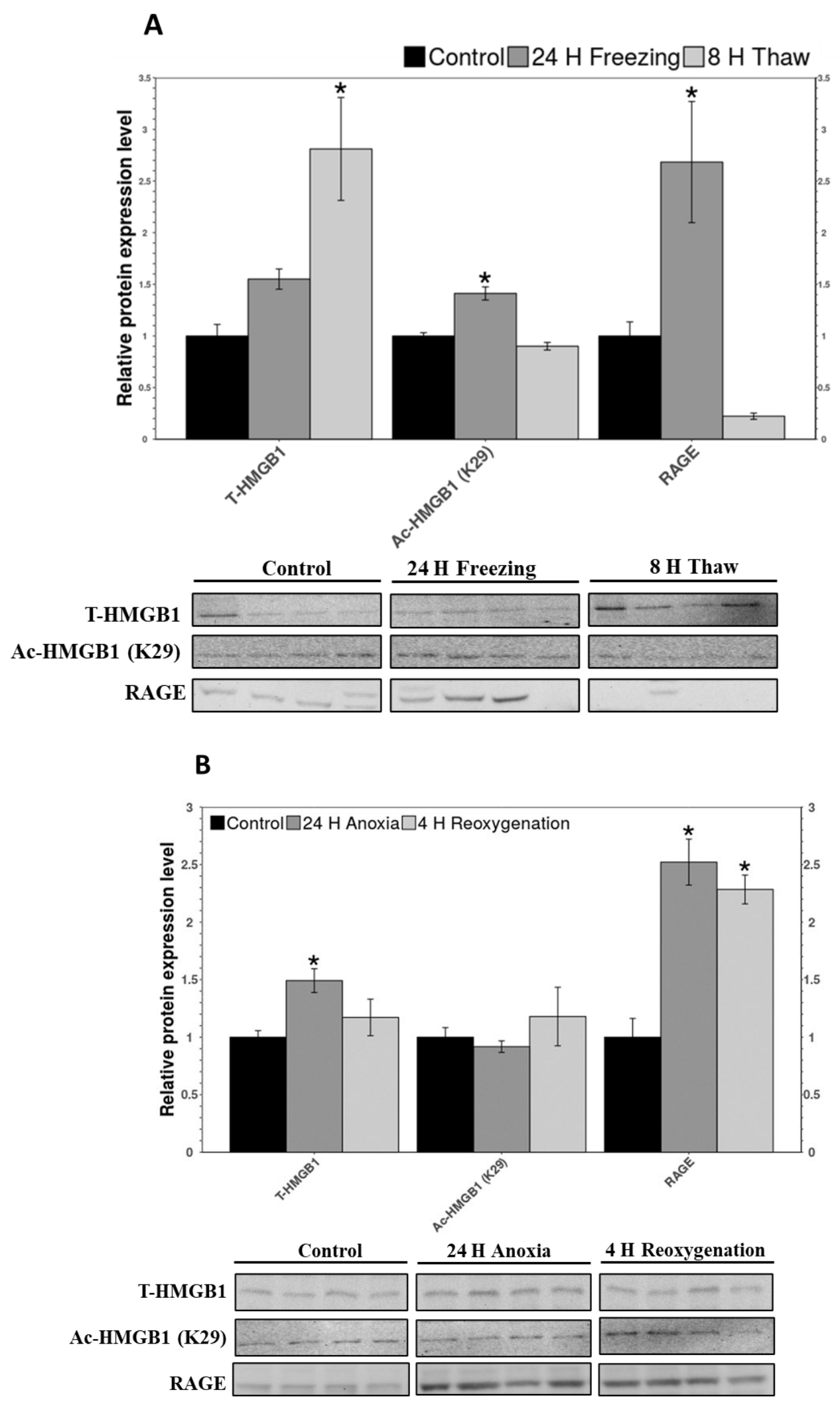


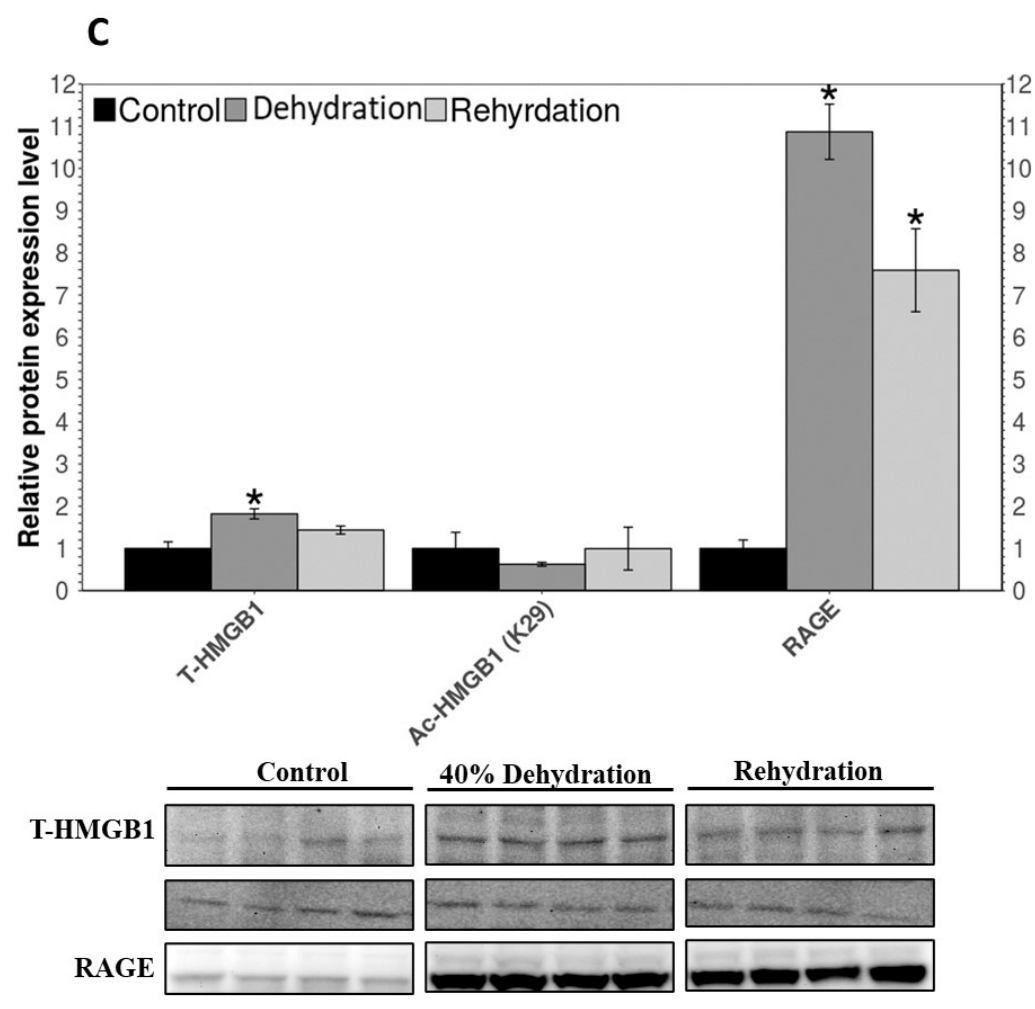

Figure 3.2: Relative protein expression of HMGB1, Ac-HMGB1 (K29) and RAGE measured in liver using immunoblotting. Measurements were done on liver from wood frogs exposed to A) freeze/thaw, B) anoxia/reoxygenation, and C) dehydration/rehydration treatments. Histogram represents mean \pm SEM of $n=4$ independent biological replicates. Significance from the control $(\mathrm{P}<0.05)$ is denote by $(*)$ as calculated using a One-Way ANOVA with a Dunnett post-hoc test. 


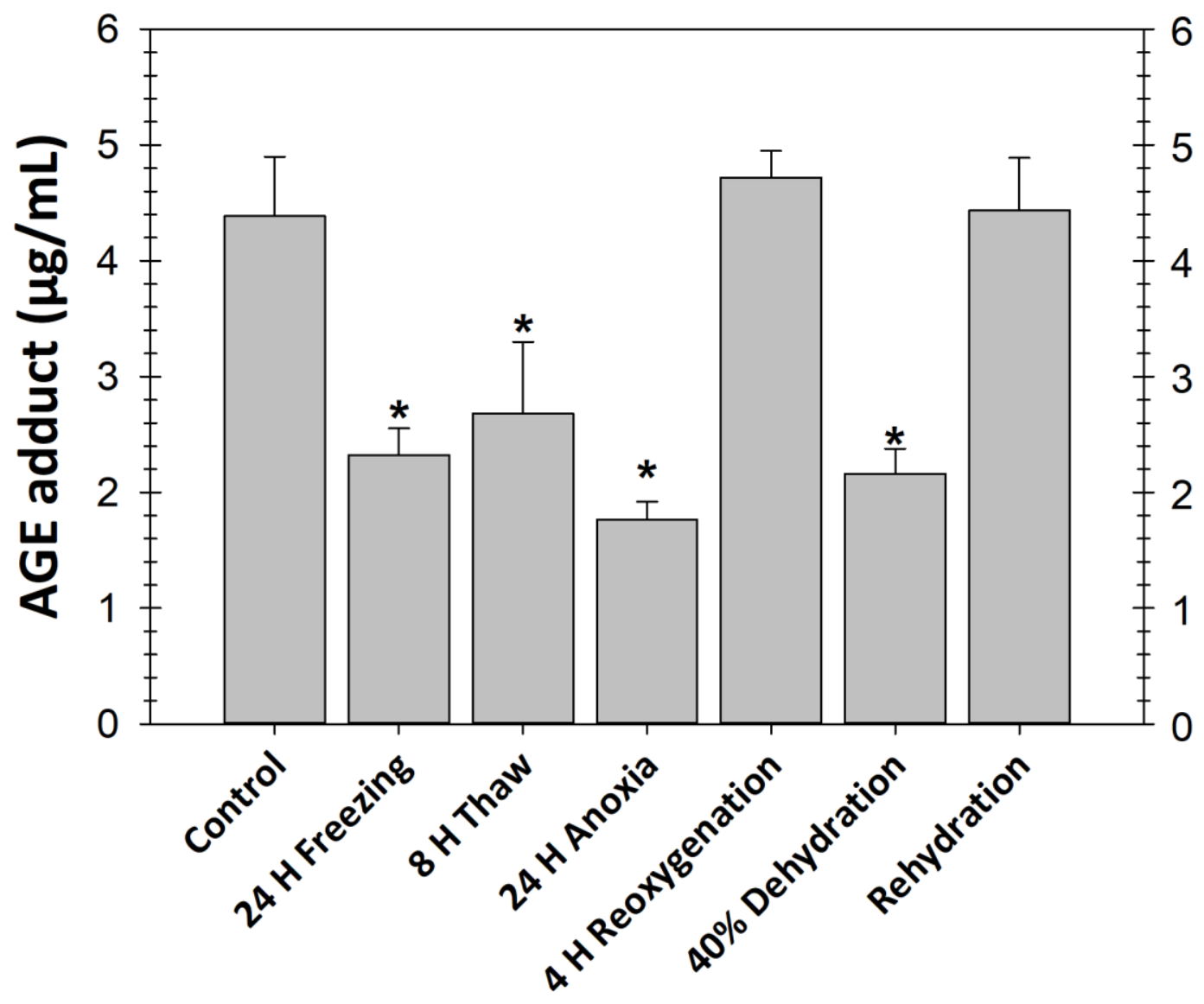

Figure 3.3: Levels of AGE-adducts present in livers measured using the OxiSelect ${ }^{\mathrm{TM}}$ Advanced Glycation End Product (AGE) Competitive ELISA Kit. Measurements were done using liver from wood frogs exposed to A) freeze/thaw, B) anoxia/reoxygenation, C) dehydration/rehydration treatments. For more information, please see Figure 3.2. 


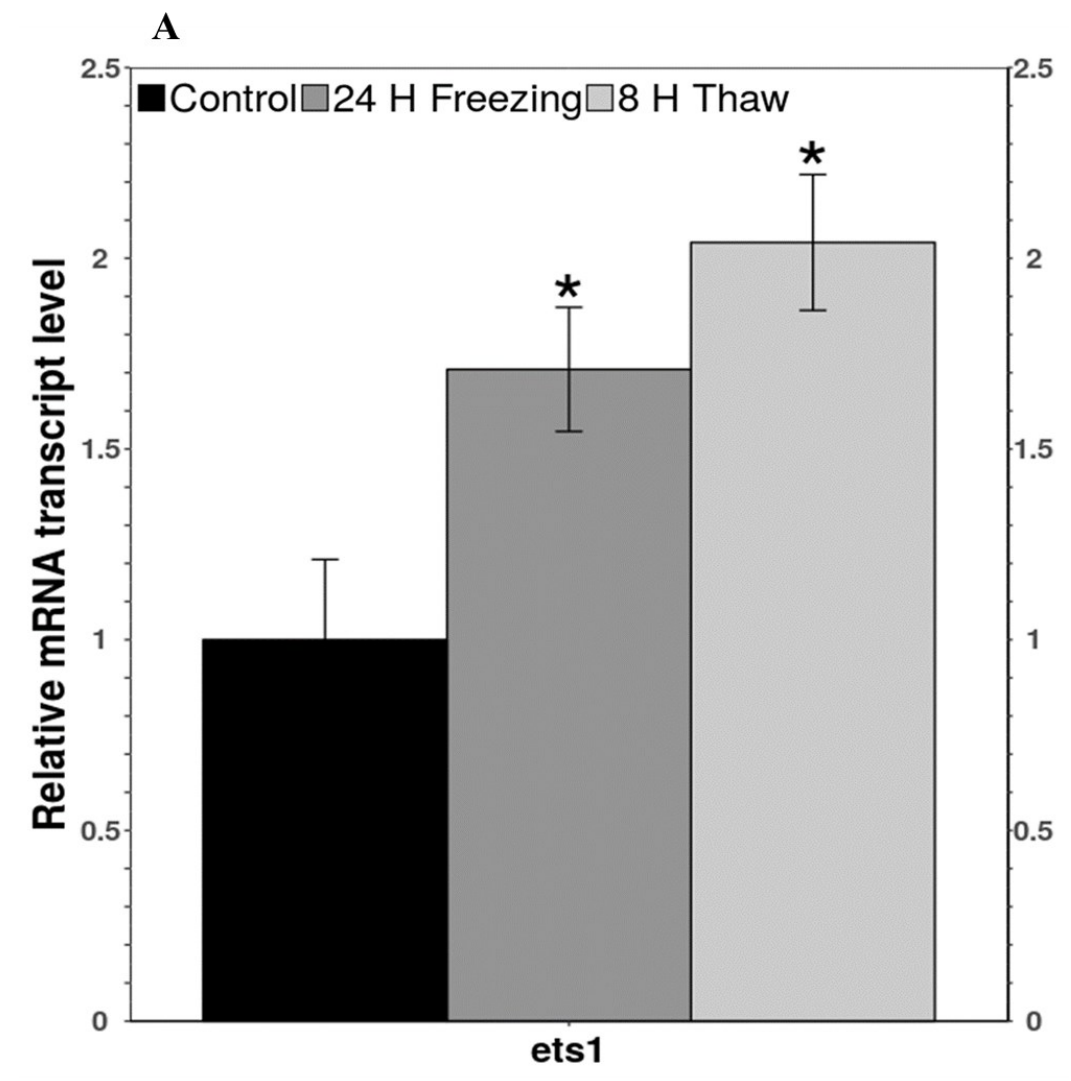

B

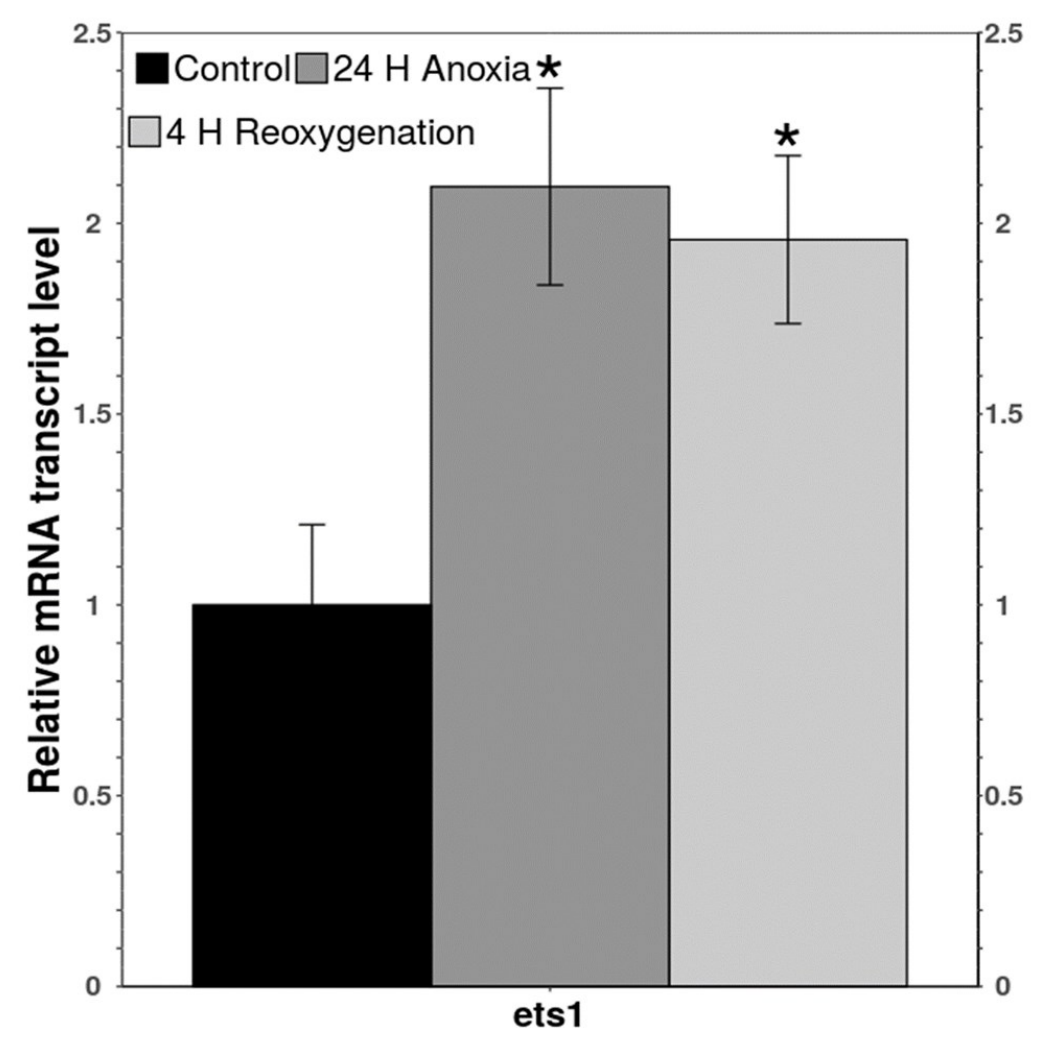




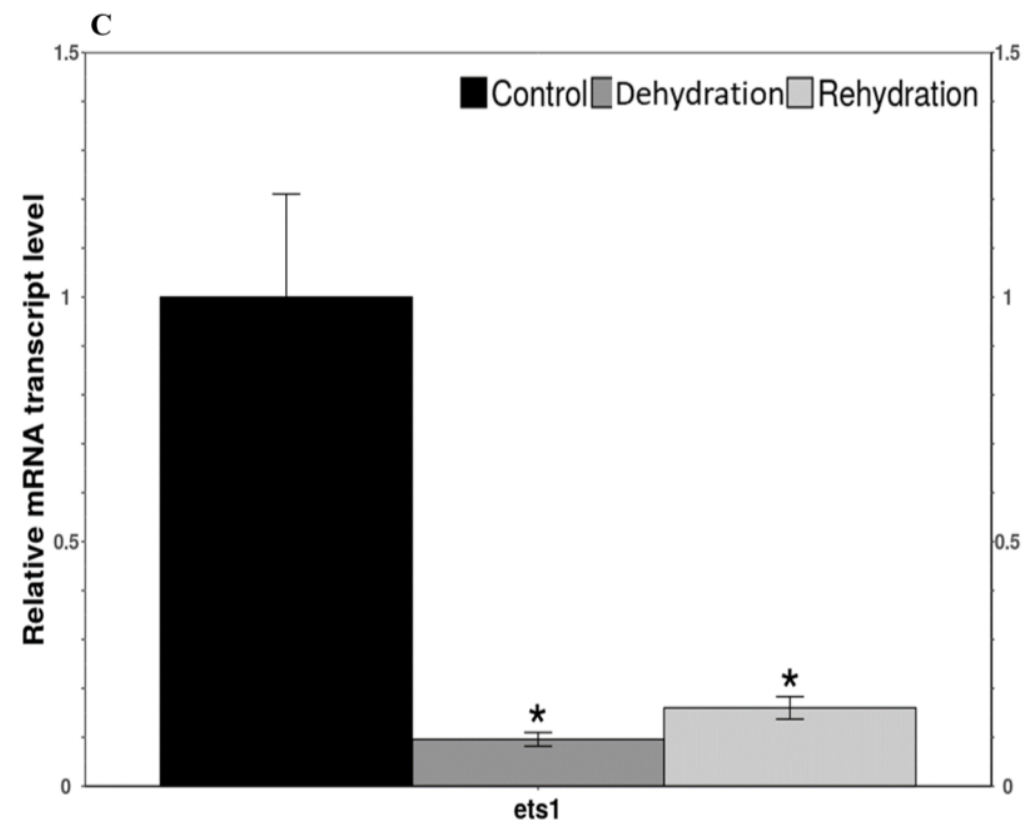

Figure 3.4: Relative transcript levels of ets 1 in liver measured by RT-qPCR. Measurements were done in liver of wood frogs expose to A) freeze/thaw, B) anoxia/reoxygenation, C) dehydration/rehydration treatments. For more information, please see Figure 3.2. 
A
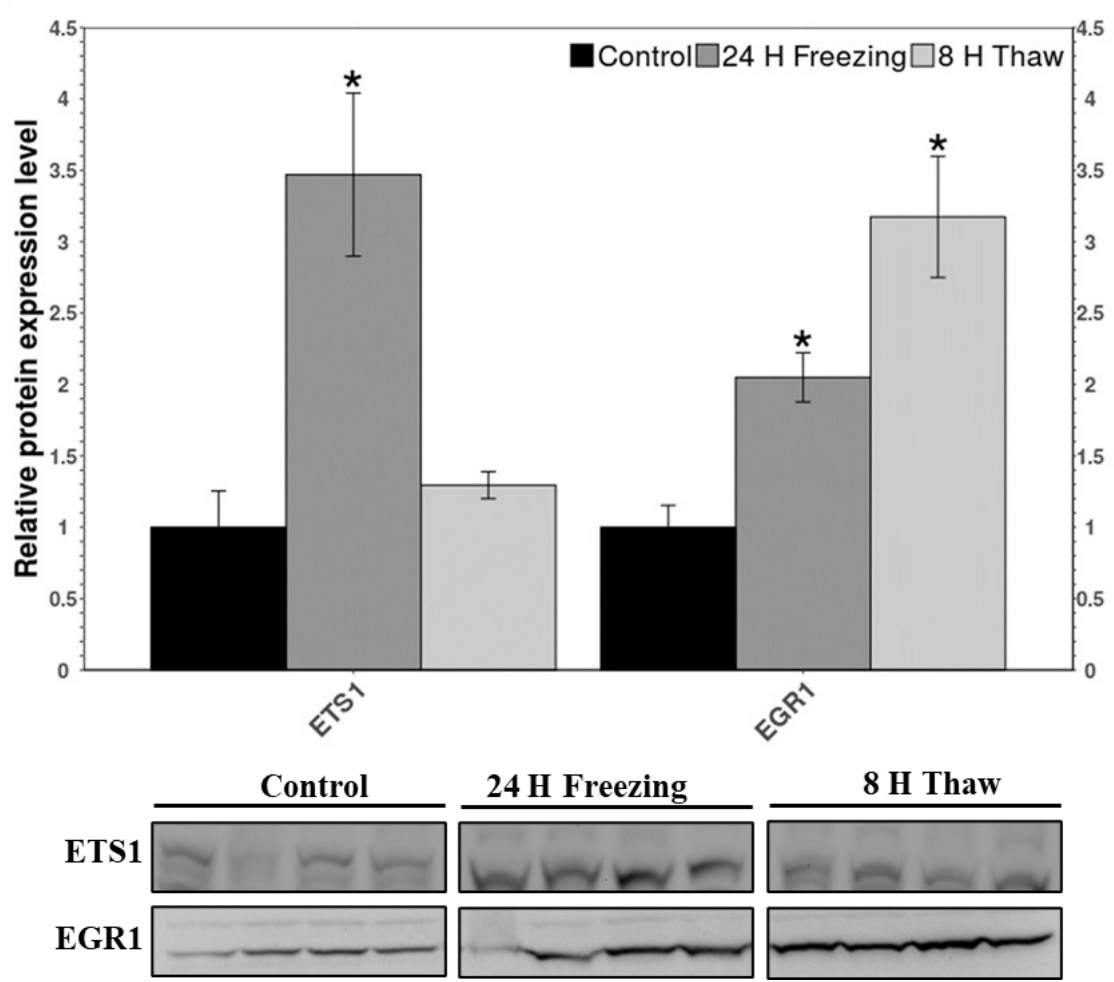

B
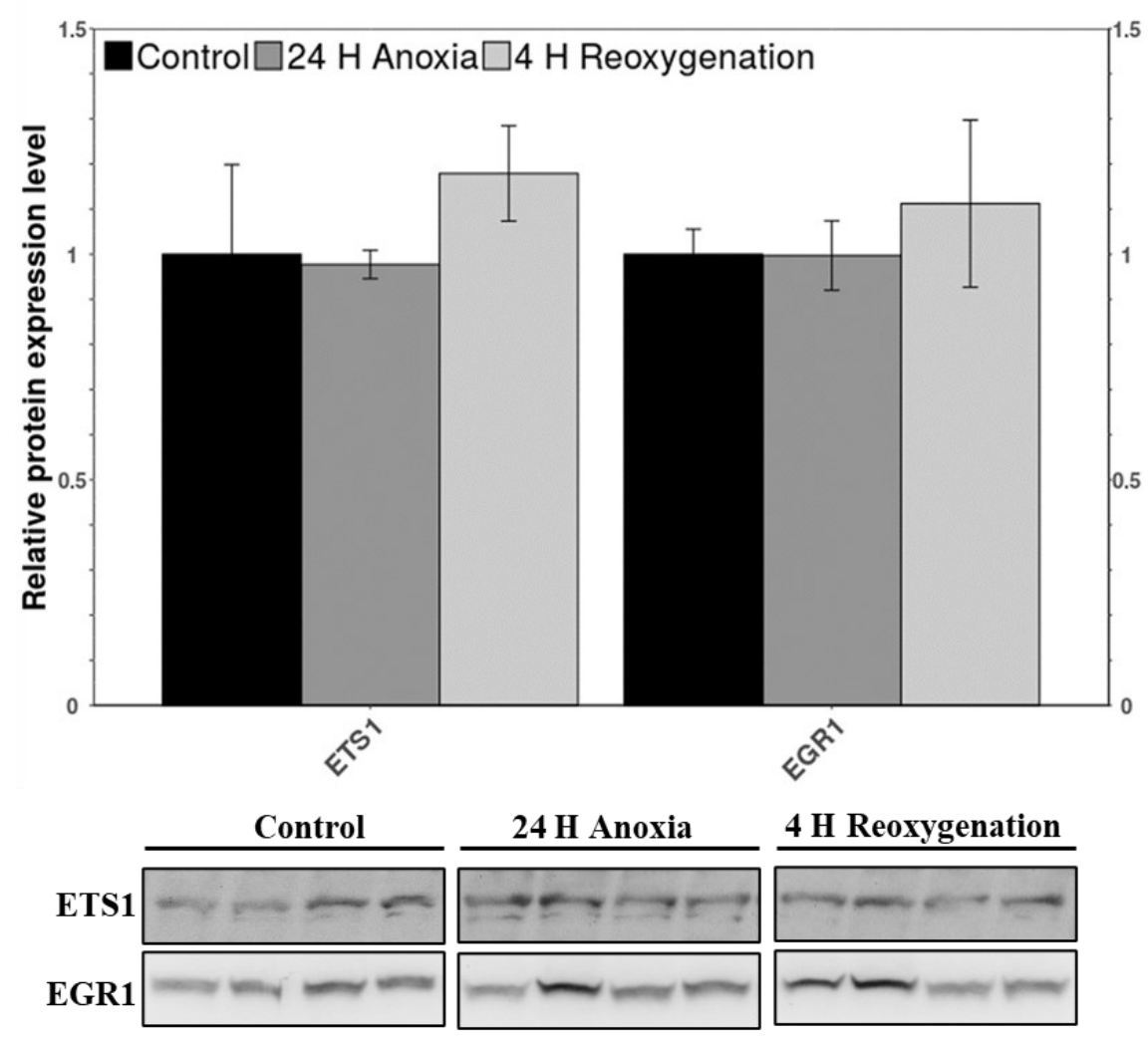


\section{C}
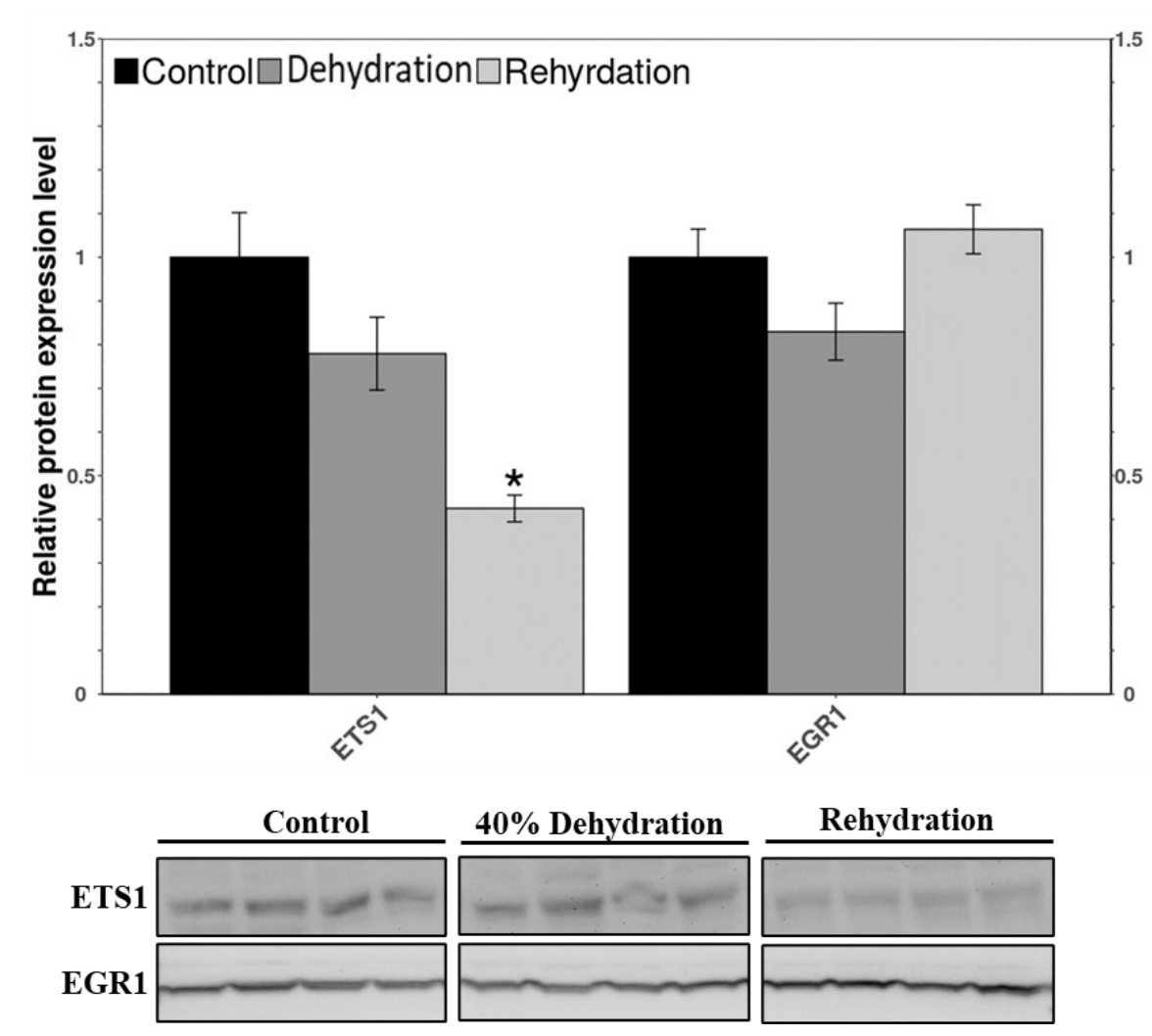

Figure 3.5: Total protein levels of ETS1 and EGR1 in liver measured using immunoblotting. Measurements were done using liver of wood frogs expose to A) freeze/thaw, B) anoxia/reoxygenation, C) dehydration/rehydration treatments. For more information, please see Figure 3.2. 
A
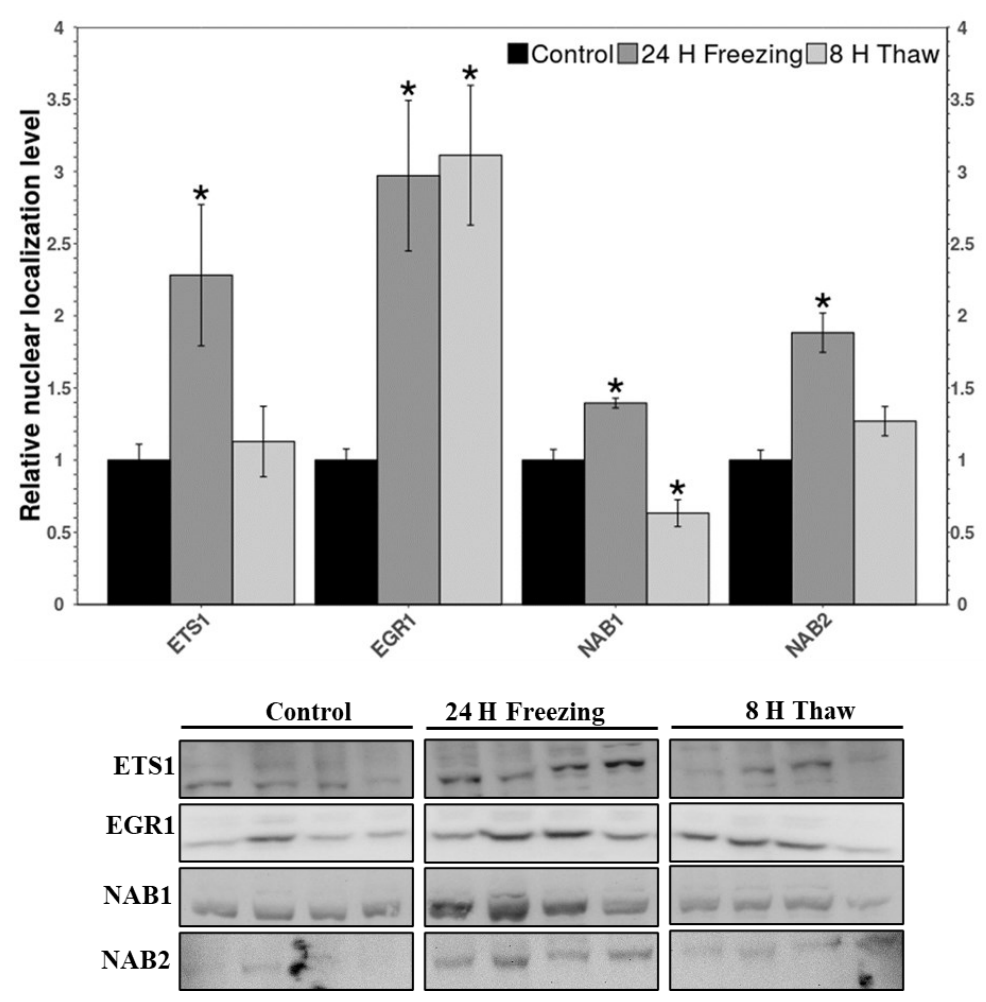

B
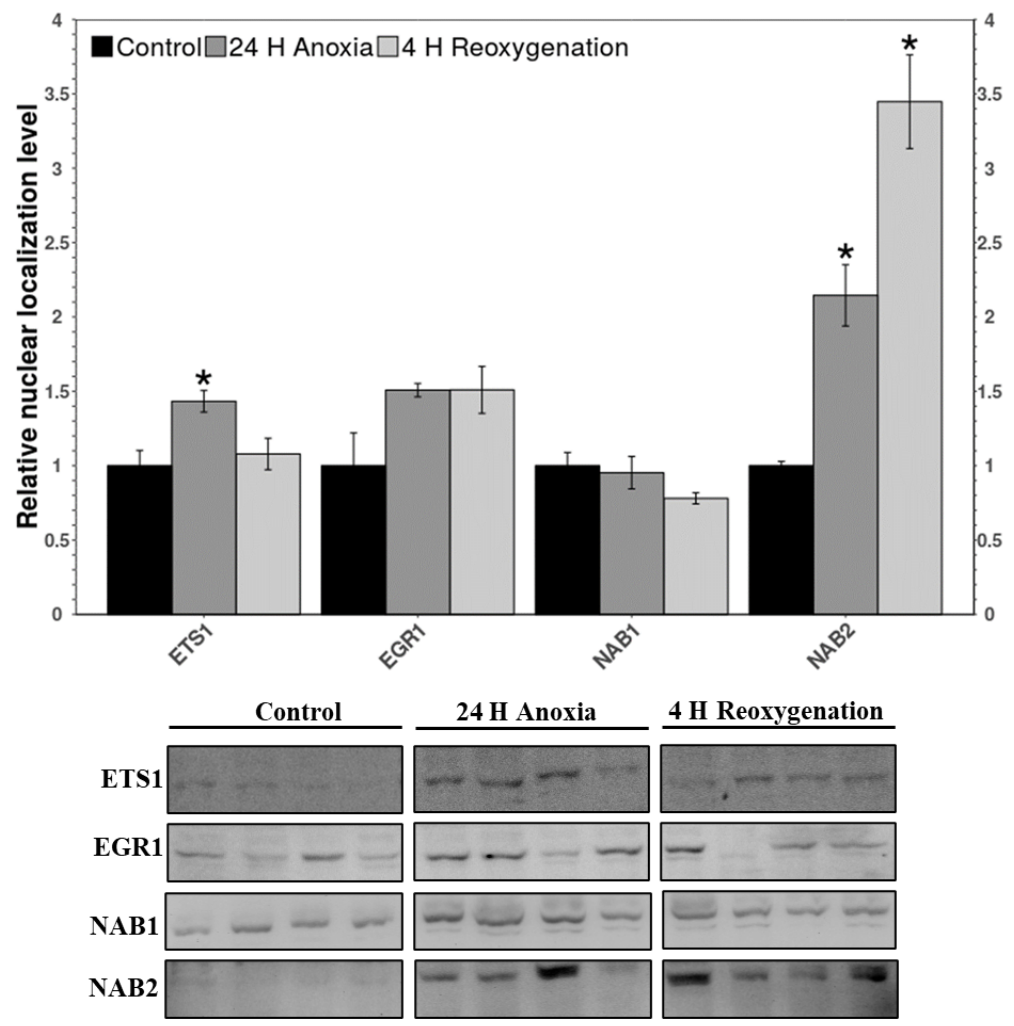


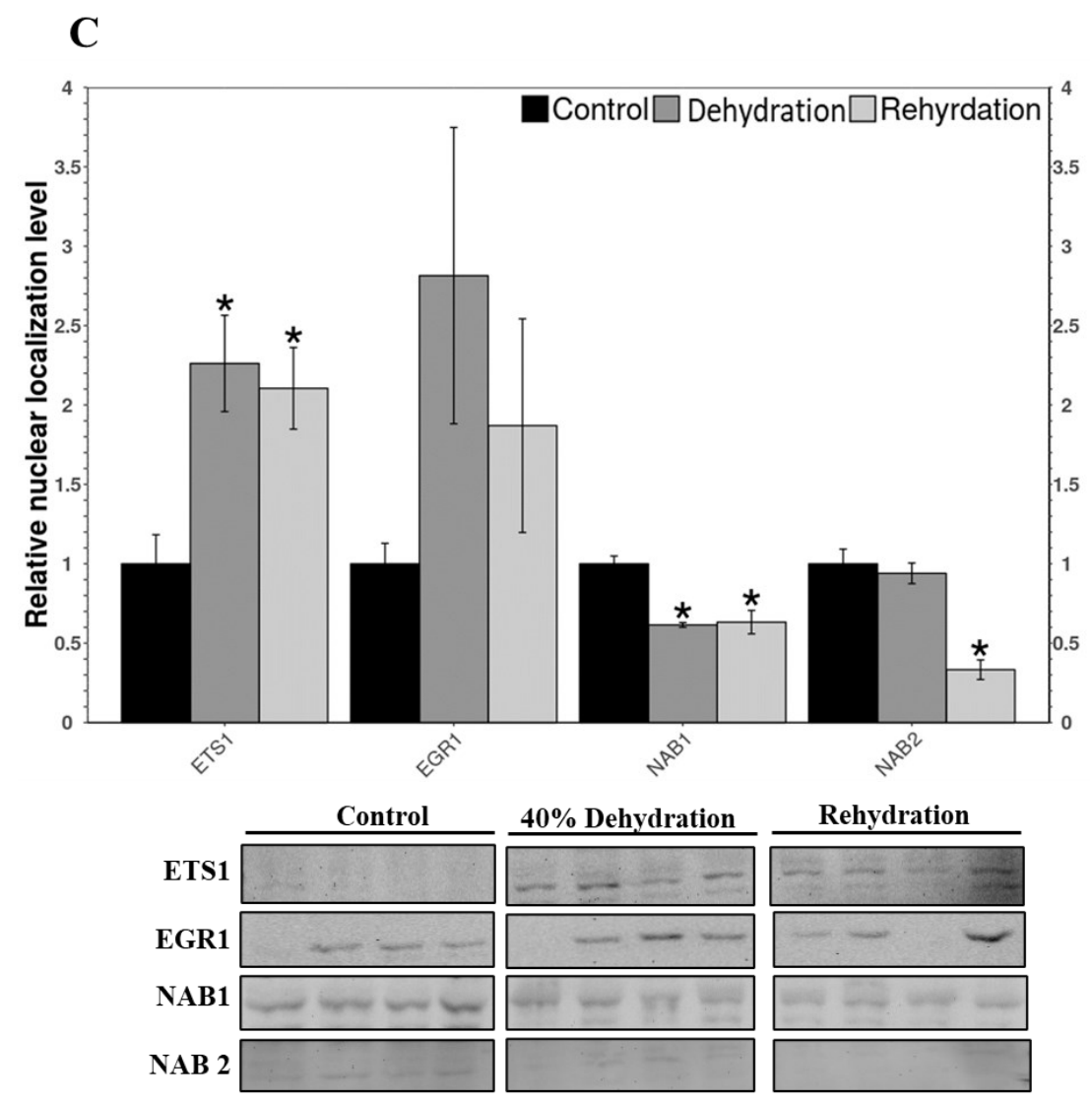

Figure 3.6: Nuclear localization levels of ETS1, EGR1 and two of EGR1 co-repressors $\mathrm{NAB} 1$ and NAB2 in liver measured using immunoblotting. Measurements were done in liver of wood frogs exposed to A) freeze/thaw, B) anoxia/reoxygenation, C) dehydration/rehydration treatments. For more information, please see Figure 3.2. 

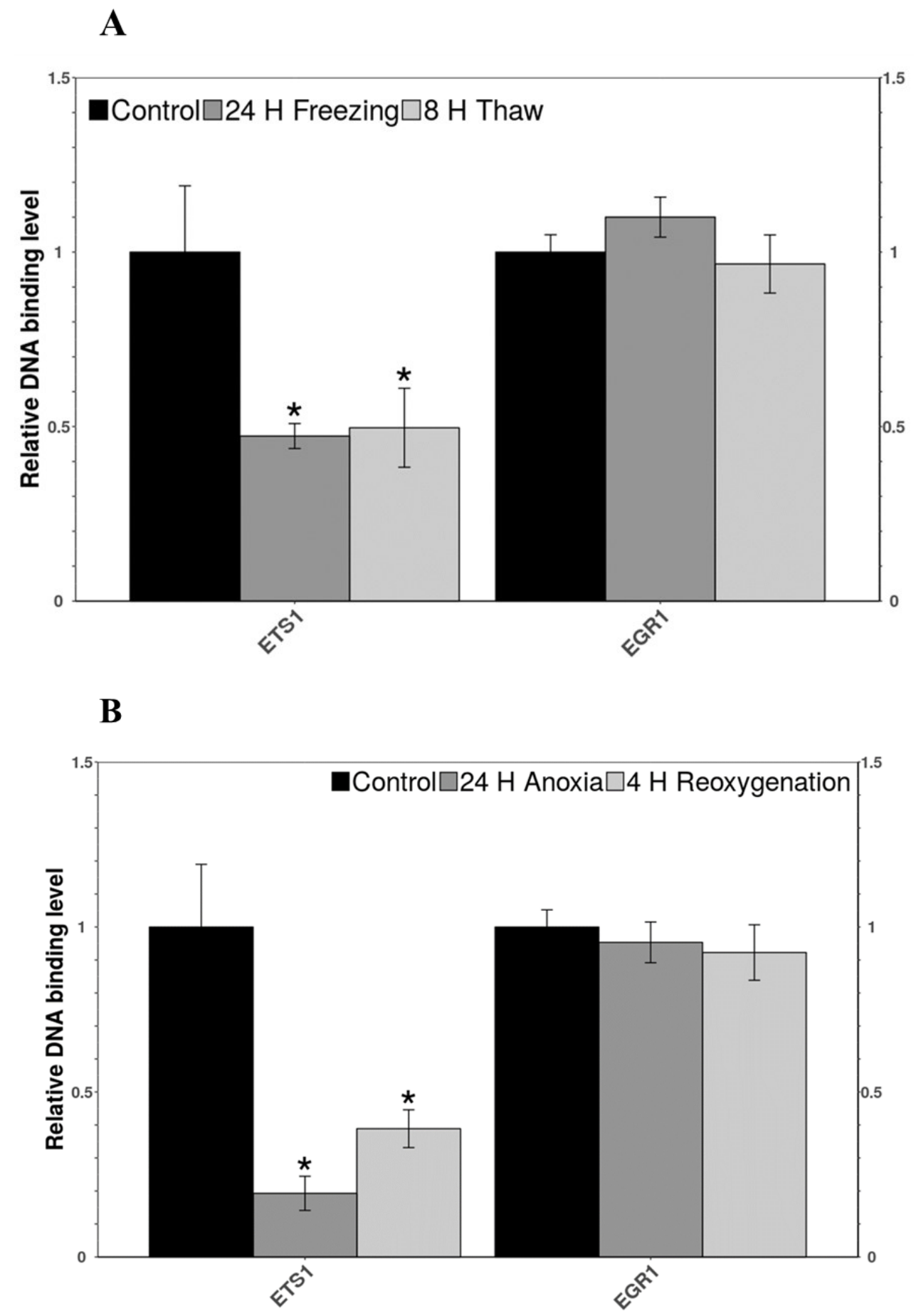


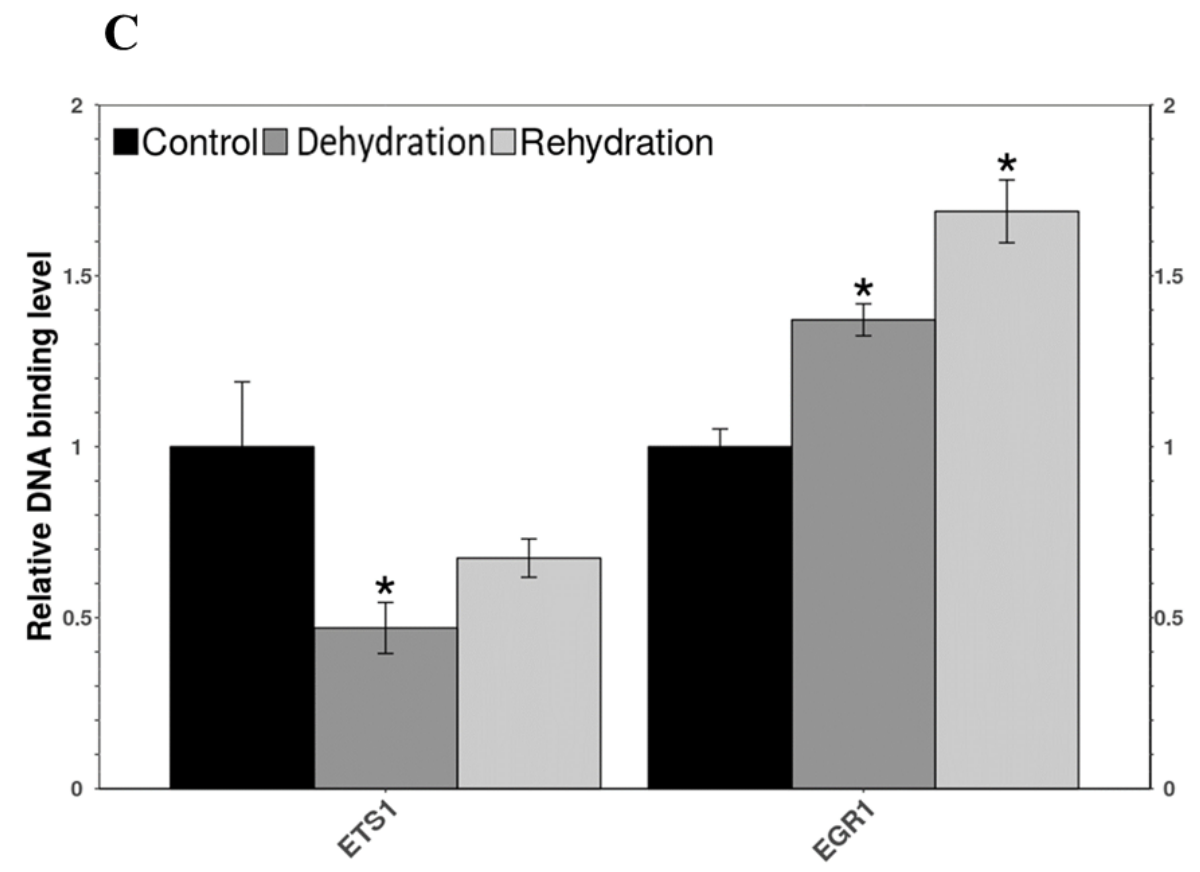

Figure 3.7: Relative DNA binding activity of ETS1 and EGR1 to their consensus sequence in liver measured by DPI-ELISA. Measurements were done using liver of wood frogs expose to A) freeze/thaw, B) anoxia/reoxygenation, C) dehydration/rehydration treatments. For more information, please see Figure 3.2. 

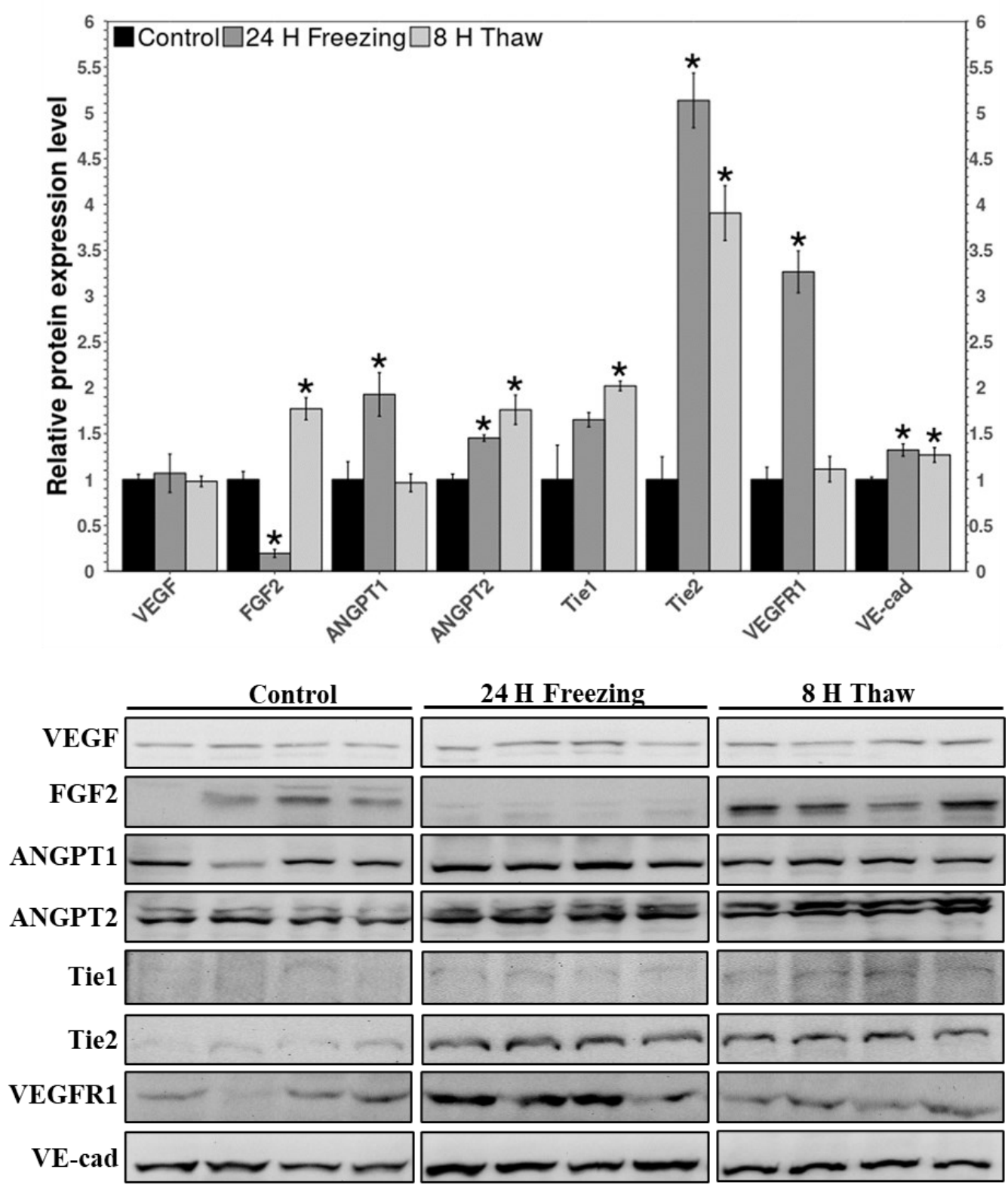

Figure 3.8: Relative protein levels of angiogenic factors in liver from wood frogs exposed to freeze/thaw treatments measured by immunoblotting. For more information, please see Figure 3.2. 


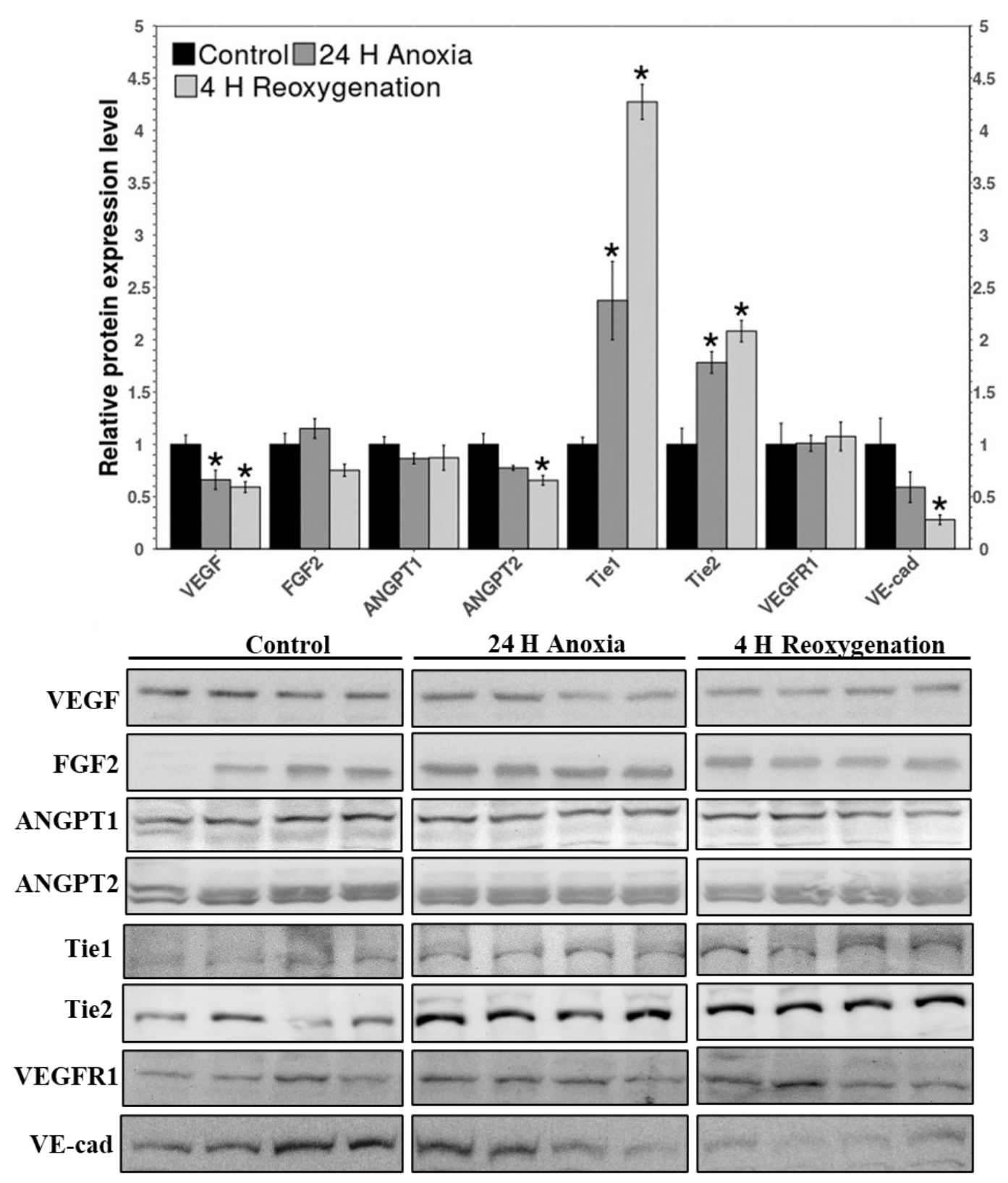

Figure 3.9: Relative protein levels of angiogenic factors in liver of wood frogs exposed to anoxia/reoxygenation treatments measured by immunoblotting. For more information, please see Figure 3.2. 

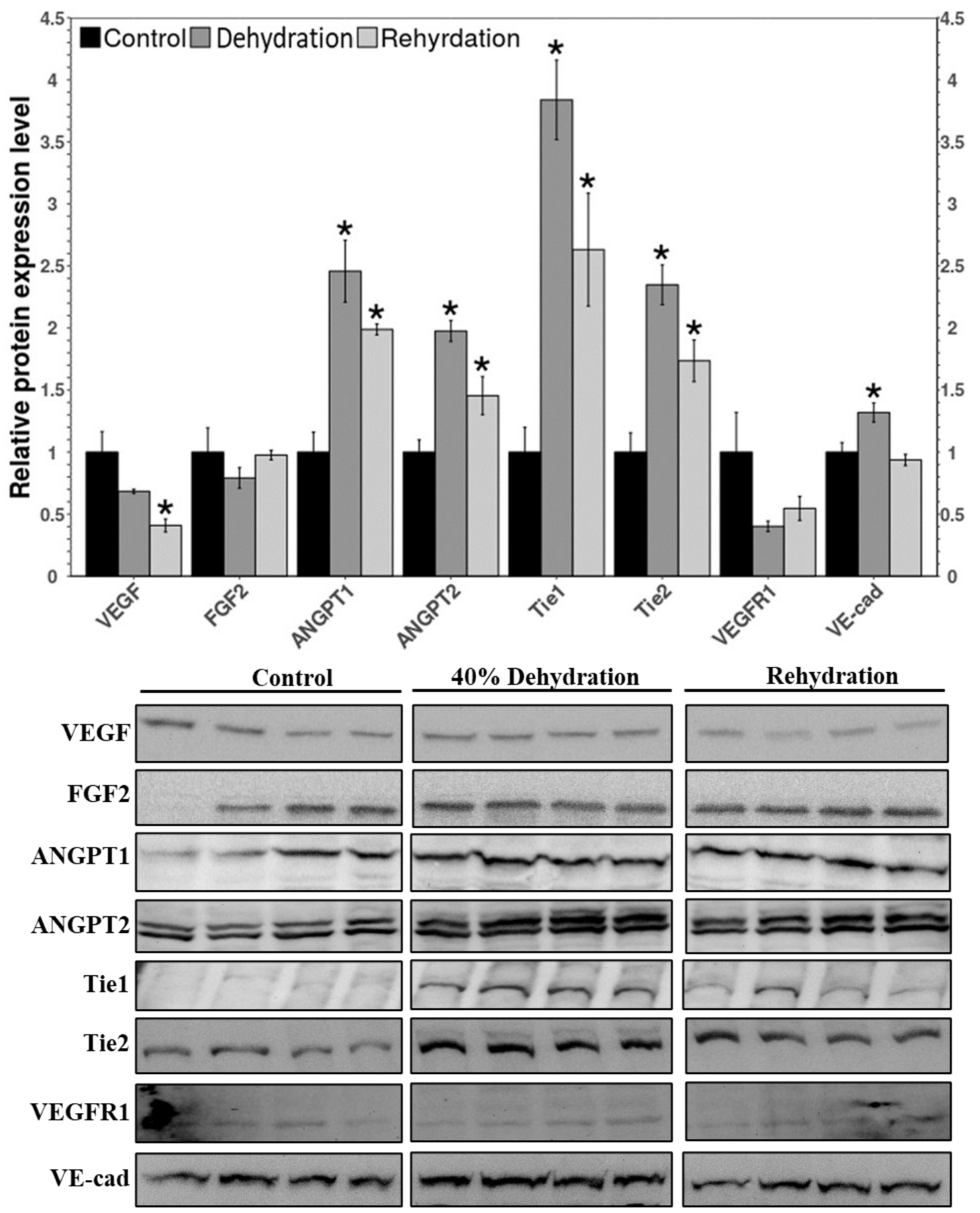

Figure 3.10: Relative protein levels of angiogenic factors in liver of wood frogs expose to dehydration/rehydration treatments measured by immunoblotting. For more information, please see Figure 3.2. 


\section{Chapter 4: RAGE against the stress: mitochondrial suppression in hypometabolic hearts}




\section{RAGE against the stress: mitochondrial suppression in hypometabolic hearts}

Rasha Al-attar ${ }^{1}$, Kenneth B. Storey ${ }^{1}$

${ }^{1}$ Institude of Biochemistry and Department of Biology, Carleton University, Ottawa, ON, Canada (K1S-5B6)

Corresponding Author:

Kenneth B. Storey

Kenstorey@cunet.carleton.ca

Telephone: +1 (613) 520-2600 x3678

Keywords: Metabolic rate depression, RAGE, AGE, ETS1, mitochondria, transcription, wood frog

This study has been published in Gene.

R. Al-Attar, K.B. Storey, RAGE against the stress: Mitochondrial suppression in hypometabolic hearts., Gene. 761 (2020) 145039.

https://doi.org/10.1016/j.gene.2020.145039. 


\subsection{Abstract}

The wood frog (Rana sylvatica) can tolerate full body freezing in winter. As a protective response, wood frogs dehydrate their cells and accumulate large quantities of glucose as an intracellular cryoprotectant. Freezing causes ischemia since blood delivery to organs is interrupted. Fascinatingly, wood frogs can tolerate dehydration, extreme hyperglycemia, and anoxia independently of freezing. In response to low oxygen levels, wood frogs strategically reduce their metabolic rates and allocate the finite amount of intracellular fuel available to pro-survival processes while reducing or interrupting all others. In this study, the involvement of advanced glycation end products (AGEs) and the high mobility group box 1 (HMGB1) protein in activating RAGE (AGE receptor) were investigated. The results show that freezing, anoxia and dehydration induced the expression of total HMGB1 and its acetylation in the heart. RAGE levels were induced in response to all stress conditions, which resulted in differential regulation of the ETS1 transcription factor. While the nuclear localization of total ETS1 was not affected, the DNA binding activity of total and its active form increased in response to freezing and dehydration but not in response to anoxia. Current results indicate that ETS1 acts as a transcriptional activator for peroxiredoxin 1 in response to freezing but acts as a transcriptional repressor of several nuclear-encoded mitochondrial genes in response to all stresses. Altogether, current results show that the HMGB1/RAGE axis may activate ETS1 in a stress-dependent manner and that activation could result in both transcriptional activation and/or repression in a stress-dependent manner. 


\subsection{Introduction}

Animals in the wild are faced with various unfavorable environmental conditions (e,g, cold temperatures, food scarcity, limited oxygen availability, droughts) that they must overcome in order to survive. The freeze-tolerant wood frog (Rana sylvatica), is one such animals that deals with cold temperatures by using a freeze-tolerant strategy [1]. These incredible animals can endure the freezing of up to $65-70 \%$ of their body water, and spend weeks in a state of suspended animation characterized by the cessation of heartbeat, breathing, muscle movement or brain conductivity, but resume normal functions once thawed [1]. The onset of freezing in wood frogs triggers the conversion of liver glycogen to glucose, and the distribution of huge quantities of this sugar to all organs in the body to be used as a cryoprotective agent [2]. In fact, blood glucose levels can rise from $5 \mathrm{mM}$ under normal conditions to $\sim 200-300 \mathrm{mM}$ during freezing, leaving wood frog organs in a state of extreme hyperglycemia [2]. Another cryoprotective measure used by wood frogs is their ability to endure cellular dehydration. As ice crystals start growing in extracellular spaces, water is drawn out of cells due to the rising osmolality of extracellular fluids leading to a drastic decrease in cell volume that is partly mitigated by the production and/or uptake of glucose as a counteracting osmolyte [3]. Secession of cardiac activity and the freezing of blood plasma ultimately halts oxygen delivery to organs, thereby leaving them in an anoxic state and necessitating a shift from aerobic to anaerobic metabolism for ATP production [4]. Given that the amount of energy generated anaerobically is far less than the amount generated under aerobic conditions, wood frogs also use metabolic rate depression (MRD) to minimize ATP and fuel consumption over what can be many weeks or months in the frozen state during the winter [1]. MRD is a finely tuned and a highly complex phenomenon where most energy 
expensive (e.g. the cell cycle) or detrimental processes (e.g. apoptosis) are suppressed, whereas pro-survival processes such as the cytoprotective and antioxidant responses are enhanced [5-7] (Figure 1).

Interestingly, wood frogs can tolerate hyperglycemia, dehydration, and anoxia independently of freezing, and thus they provide a unique model for deciphering the mechanisms of freeze tolerance in vertebrates. The accumulation of extreme amounts of glucose as a cryoprotective agent, occurring especially in response to freezing and dehydration (and to a lesser degree under anoxia) could result in glucose-induced cytotoxicity and damage to proteins. Indeed, high levels of glucose can result in nonenzymatic glycation of macromolecules where glucose moieties are covalently attached to the free amino groups of N-terminal amino acids, arginine or lysine residues in a nonenzymatic manner. Upon further processing these molecules are converted into highly stable advanced glycation end products (AGEs) [8]. AGEs can interact with various amino acid residues within a protein to generate protein crosslinks or AGE-protein adducts [8]. Generation of AGEs is a slow process and can take from months to years to accumulate and therefore most proteins that are affected by AGEs are long-lived proteins such as collagen. However, conditions that favor hyperglycemia or oxidative stress can reduce the time of AGE formation to a few hours, thereby also affecting the glycation of short-lived macromolecules [9].

High levels of glucose during freezing in wood frogs did not cause a change in glycated serum albumin levels; however, levels had increased 7 hours post thaw [10]. The same study also reported that levels of glycated hemoglobin decreased in response to freezing and remained low after 7 days of thawing. Interestingly, in vitro treatment of 
whole blood from wood frogs, leopard frogs and rats with $0.4 \mathrm{M}$ glucose resulted in an increase in glycated hemoglobin levels in all species, albeit levels in leopard frogs and wood frogs were higher than in the rat [10]. Altogether, these results suggest that perhaps wood frogs have a unique in vivo mechanism that prevents or reduces protein glycation in response to freezing. AGE-adducts and other secreted molecules can initiate cascades of events after binding to the receptor for AGE (RAGE) [11]. Another ligand recognized by RAGE is the high mobility group box 1 (HMGB1) protein [12]. Under normal conditions, this non-histone DNA binding protein binds chromatin structures to maintain their integrity; however, in response to oxidative stress, HMGB1 becomes acetylated and is exported to the cytoplasm and the plasma membrane [13]. Indeed, HMGB1 is engulfed in secretory vesicles and is exported into the extracellular space where it can bind to RAGE and other cell surface receptors such as toll-like-receptors to induce a cascade of events $[14,15]$. Previous cDNA array screening by our group demonstrated that levels of RAGE are significantly upregulated in response to freezing in wood frog heart [16]. Therefore, we investigated whether exposure to freezing, anoxia and dehydration increases RAGE ligands and if so, can RAGE increase transcriptional responses to promote adaptation under these conditions.

Binding of ligands to RAGE on the surface of the cell triggers the recruitment and association of diaphanous-1 (mDia-1) with the cytoplasmic domain of RAGE [17]. mDia1 was shown to be necessary for the activation of protein kinase C $\beta I I$ (PKC $\beta I I)$, its membrane association and subsequent activation of JNK and ERK1/2 kinases under hypoxic conditions [17,18]. Activated ERK1/2 can then phosphorylate the E26 transformation-specific sequence 1 (ETS1) transcription factor that results in its nuclear 
translocation from the cytoplasm [19]. ETS1 regulates numerous genes involved in angiogenesis, proliferation, autophagy, apoptosis, antioxidant defense, and energy metabolism, to name a few [20-24]. The ETS family of transcription factors has also been reported to act as transcriptional repressors [25]. Indeed, ETS1 overexpression in goldfish (a highly anoxia tolerant species) was shown to suppress the transcription of nicotinic acetylcholine receptor epsilon-subunit in muscle cells [26]. Moreover, overexpression of ETS1 was also shown to coincide with reduction in the expression level of several proteins involved in the electron transport chain including, but not limited to, NADH dehydrogenase (ubiquinone 1) $\alpha / \beta$ subcomplex 1 (NDUFAB1), cytochrome c1 (CYC1), and ATP synthase $\mathrm{H}^{+}$transporting mitochondrial F1 complex, $\alpha$ subunit 1 cardiac muscle (ATP5A1) $[23,24]$. All three proteins play a significant role in promoting mitochondrial bioenergetics and dysregulation of their expression could manifest in mitochondrial stress in mammalian cells [27-29]. Aside from mitochondrial bioenergetics, ETS1 is also involved in regulating the expression of a network of genes involved in glucose uptake and metabolism [23,24]. For example, overexpression of ETS1 was shown to reduce the expression of citrate synthase (CS), an important enzyme that initiates the tricarboxylic acid cycle (TCA) [23,24]. By contrast, ETS1 was also shown to promote the expression of glucose transporter 1 (GLUT1) [30]. This transporter localizes on the plasma membrane, is ubiquitous in most tissues including the heart, and is responsible for basal glucose uptake by cardiac cells [31].

The present study examines the involvement of AGE and HMGB1 in regulating the activity of the ETS1 transcription factor in wood frogs dealing with environmental stress (freezing, anoxia, dehydration). Current results demonstrate that the 
HMGB1/RAGE axis leads to the differential regulation of ETS1 in a stress-dependent manner. Furthermore, the DNA binding activity of active ETS1 can lead to transcriptional activation of PRX1 in response to freezing, but potentially inhibit PRX5 in response to dehydration and AOX1 in response to freezing and dehydration in the heart. Moreover, selected downstream targets encoding components of nuclear-encoded mitochondrial proteins, glucose uptake and metabolism appear to be negatively impacted by an increase in DNA binding by active ETS1.

\subsection{Materials and methods}

Animal treatment

Adult male wood frogs were captured from breeding ponds in the Ottawa area in early spring and transported to Carleton University. All animal experiments were performed as previously described [32,33]. Following treatment, all animals were euthanized by pithing and tissues were quickly collected and flash frozen in liquid nitrogen followed by long term storage at $-80^{\circ} \mathrm{C}$. All animal treatment protocols were

previously approved by the Carleton University Animal Care Committee (protocol \# 106935) and followed guidelines set by the Canadian Council on Animal Care.

\section{Total protein extraction}

Total protein extraction was performed as previously published [34]. The supernatant containing the soluble protein fraction was collected and protein concentration was measured using the Bradford method (Bio-Rad, catalogue no. 5000006). All samples were standardized to a constant concentration and frozen for long term storage at $-80^{\circ} \mathrm{C}$.

Cytoplasmic-nuclear fractionation 
For transcription factors to be transcriptionally active, they must localize to the nucleus. As such, nuclear localization of ETS1 was assessed by first isolating cytoplasmic versus nuclear fractions. Briefly, $\sim 50 \mathrm{mg}$ of frozen heart tissue was homogenized 1:5 (w:v) in 1x buffer A (10 mM HEPES pH 7.9, $10 \mathrm{mM} \mathrm{KCl}, 10 \mathrm{mM}$ EDTA, and $20 \mathrm{mM} \beta$-glycerol phosphate, with $10 \mu \mathrm{L}$ of $100 \mathrm{mM}$ DTT and $10 \mu \mathrm{L}$ of protease inhibitor cocktail added per $\mathrm{mL}$ of buffer) using a manual Dounce homogenizer with 5-6 strokes. All homogenates were incubated on ice for $\sim 25$ min to maximize extraction before being centrifuged at $12,000 \mathrm{rpm}$ for 15 min at $4^{\circ} \mathrm{C}$. Following centrifugation, the soluble fraction was collected and stored in at $-80^{\circ} \mathrm{C}$ as the cytoplasmic fraction. The pellet was resuspended in $\sim 250 \mu \mathrm{L}$ of buffer B (100 mM HEPES pH 7.9, $2 \mathrm{M} \mathrm{NaCl}, 5 \mathrm{mM}$ EDTA; 50\% v:v glycerol and $100 \mathrm{mM} \beta$-glycerol phosphate with $10 \mu \mathrm{L}$ of $100 \mathrm{mM}$ DTT and $10 \mu \mathrm{L}$ of protease inhibitor added per mL of buffer followed by sonication for $\sim 10$ sec before incubation on ice for $10 \mathrm{~min}$. Pellet homogenates were then centrifuged at $14,000 \mathrm{rpm}$ at $4^{\circ} \mathrm{C}$ for $10 \mathrm{~min}$. The supernatant containing the nuclear fraction was collected and stored at $-80^{\circ} \mathrm{C}$. To ensure successful fractionation, all fractions were subjected to western blot analysis. Both nuclear and cytoplasmic fractions were probed with antibodies to a nuclear only (histone H3) or cytoplasm only ( $\beta$-tubulin) protein to ensure the enrichment of each marker in its respective fraction.

\section{Western blotting}

Protein concentrations of total protein extracts and cytoplasmic/nuclear fractions were measured using the Bradford method and standardized to a constant concentration. Samples were treated and used for western blot analysis as previously described [35]. 
The following antibodies were used: HMGB1(PCRP-HMGB1-3A7, University of Iowa hybridoma bank), Ac-HMGB1 K29 (A16002, Abclonal), RAGE (GTX23611, GeneTex), PKCIIß S660 (9371, Cell Signaling), AOX1 (A3586, Abclonal), CYC1 (A10449, Abclonal), p-ETS1 T38 (E-Ab-21277, eLabScience), GLUT1 (A6982, Abclonal), PRX1 (A16412, Abclonal), PRX 5 (A1269, Abclonal), ERK2 (Sc-1647, SantaCruz), ATP5A1 (A5884, Abclonal), NDUFAB1 (A14657, Abclonal), CS (110624, GeneTex).

\section{Advanced glycation end-product (AGE) measurement}

Total levels of AGE-adducts formed under each condition was measured using a commercially available kit (OxiSelect ${ }^{\mathrm{TM}}$ Advanced Glycation End Product Competitive ELISA Kit, Cell BioLabs, catalogue no. STA-817) following a protocol provided by the manufacturer. AGE-conjugate $(10 \mu \mathrm{g} / \mathrm{mL})$ and conjugate diluent both provided by the kit were diluted to $1 \mathrm{X}$ with PBS and mixed in a 1:1 v:v ratio. A $100 \mu \mathrm{L}$ volume of the mixture was added to each well of the microplate and incubated overnight at $4^{\circ} \mathrm{C}$. Excess solution was discarded, and wells were washed with $100 \mu \mathrm{L}$ of PBS twice. A $200 \mu \mathrm{L}$ volume of assay diluent was added to each well to block unreactive sites, and the plate was incubated for $1 \mathrm{~h}$ at room temperature. Following blocking, excess assay diluent was removed, and $50 \mu \mathrm{L}$ of either heart extracts $(40 \mu \mathrm{g})$ or AGE-BSA standard was added to each well in duplicate. The concentration of total protein homogenates was again measured using the Bradford method and the concentration was standardized using phosphate buffered saline $\left(137 \mathrm{mM} \mathrm{NaCl}, 10 \mathrm{mM} \mathrm{Na}_{2} \mathrm{HPO}_{4}, 2.7 \mathrm{mM} \mathrm{KCl}\right.$, and $2 \mathrm{mM}$ $\mathrm{KH}_{2} \mathrm{PO}_{4}, \mathrm{pH}$ 7.4) containing $0.1 \% \mathrm{BSA}$. The plate was incubated at room temperature for 10 minutes with gentle shaking. Following incubation, $50 \mu \mathrm{L}$ of anti-AGE antibody was added to each well and the plate was incubated for another hour at room temperature with 
gentle agitation. Subsequently, excess solution was removed, wells were washed $3 \mathrm{x}$ with $250 \mu \mathrm{L}$ wash buffer and $100 \mu \mathrm{L}$ of secondary antibody conjugated to HRP was added to each well. The plate was incubated for $1 \mathrm{~h}$ at room temperature with gentle agitation before discarding excess secondary antibody. All wells were washed three times and incubated with a volume of $100 \mu \mathrm{L}$ of substrate solution for 10 min with gentle agitation at room temperature. Once the desired color intensity was reached, the reaction was terminated by adding $100 \mu \mathrm{L}$ of stop solution to each well. The plate was read at $450 \mathrm{~nm}$, and the amount of AGE-adducts in the sample was calculated using the standard curve generated.

DNA-protein interaction enzyme linked immunosorbent assay (DPI-ELISA)

The DNA binding activity of total ETS1 and p-ETS1 T38 (one of the active forms of ETS1) to the ETS consensus sequence was measured using a DPI-ELISA as previously explained [35]. DNA oligonucleotides containing the ETS1 consensus sequence (forward, 5'-Biotin-GATCTCGAGCAGGAAGTTCGA-3', complementary 3'CTAGAGCTCGTCCTTCAAGCT-5') were generated by Integrated DNA Technologies (Coralville, IA, USA). Primary antibodies (anti-ETS1 and anti-p-ETS1 T38) were diluted to 1:1000 and secondary antibody (anti-rabbit HRP) was diluted to 1:2000, both in PBST (PBS containing $0.05 \%$ Tween-20). The specificity of the probe for the ETS1 transcription factor was previously tested by performing the same assay in wells lacking either the probe, primary antibody, or the protein. Using a dilution curve of pooled heart samples, it was determined that the optimum concentration to be used in the assay is 25 $\mu \mathrm{g}$ of protein per well.

Comparison of the 3-dimentional structure of ETS1 
Many studies referenced here rely on findings in mammals, and non-amphibian models. Therefore, to ensure structural similarities between amphibian and mammalian ETS1, the protein sequence of Xenopus tropicalis (NP_001123840_1) and Human (P14921.1) was used to predict the 3-dimentional structure of this protein using the Protein Homology/Analogy Recognition Engine V2.0 (Pyre ${ }^{2}$ ) (http://www.sbg.bio.ic.ac.uk/phyre2/html/page.cgi?id=index). The generated files were then analyzed using the PyMOL Molecular Graphics System, Version 2.0 Schrödinger, LLC.

\section{Quantification and statistics}

Band intensities on membranes corresponding to the enhanced chemiluminescent signal and the corresponding Coomassie-blue stained membrane were quantified using GeneTools (Syngene, Frederick, MD, USA). Band intensities of enhanced chemiluminescence signals were standardized against the intensity a group of proteins in each lane that showed stable expression across all lanes; this was used to account for any loading variations that may have occurred. This method was shown to be more accurate than using a single housekeeping protein [36]. Changes in protein expression or localization were reported as fold-change relative to the control, which was set to 1 . For DPI-ELISA and AGE-adduct measurement, relative intensities of microplate well were measured at $450 \mathrm{~nm}$, with a reference wavelength of 655 . The relative DNA binding activity of total ETS1 and p-ETS1 T38 under different conditions was reported with respect to the control group which was set to 1. The amounts of AGE-adducts in hearts under each condition were calculated by converting the absorbance values into concentrations using an AGE-BSA standard curve. All histograms are presented as mean 
relative protein expression, protein localization or DNA binding activity \pm SEM for $n=4$ independent biological replicates. Statistical testing used a One-Way ANOVA with a Dunnett's post-hoc test. Significant difference from control was accepted if $P<0.05$. The SigmaPlot software (SYSTAT, San Jose, CA, US) was used for statistical analysis and graphical presentation [37].

\subsection{Results}

Expression levels of AGE-adducts and protein levels of RAGE and its ligands and downstream factors

Levels of AGE-adducts in hearts decreased down to $3.05 \pm 0.3 \mu \mathrm{g} / \mathrm{mL}$ in response to $24 \mathrm{~h}$ of freezing at $-2.5^{\circ} \mathrm{C}$, a significant reduction from the $4.5 \pm 0.3 \mu \mathrm{g} / \mathrm{mL}$ for heart from $5^{\circ} \mathrm{C}$ acclimated controls (Figure 2). However, despite showing a decreasing trend, there was no significant change in AGE-adduct levels in response to anoxia or dehydration. Relative total and post-translationally modified levels of different proteins were measured using western blotting. Levels of total HMGB1 (T-HMGB1) increased by $2.7 \pm 0.24$-fold, $2.7 \pm 0.07$-fold, and $2.2 \pm 0.21$-fold following freezing, anoxia and dehydration treatments (Figure 3). Levels of acetylated HMGB1 (Ac-HMGB1 K29) also increased in response to all treatments with levels rising by $4.8 \pm 0.34$-fold, $4.7 \pm 0.30$ fold, and $2.0 \pm 0.17$-fold in response to freezing, anoxia and dehydration, respectively. Total protein levels of RAGE, the receptor that binds AGEs and HMGB1 also increased under all conditions. RAGE levels increased by $1.7 \pm 0.12$-fold following freezing, by 1.9 \pm 0.08 -fold following anoxia and by $2.6 \pm 0.23$-fold following dehydration exposure. The phosphorylated form of PKCII $\beta$ (S660) did not show a statistically significant change in response to freezing or anoxia, but levels increased by $2.2 \pm 0.38$-fold in response to 
dehydration. Total levels of ERK2 remained the same in response to freezing, decreased by $33 \pm 7 \%$ in response to anoxia, and increased by $1.8 \pm 0.23$-fold following dehydration treatment. The total protein levels of ETS1 did not change in response freezing or anoxia but decreased by $22 \pm 6 \%$ after dehydration. The phosphorylated form of p-ETS1 (T38) also showed no change in response to freezing or anoxia but showed a significant increase by $2.8 \pm 0.57$-fold in response to dehydration.

Nuclear localization of ETS1 and DNA binding activity of ETS1

Levels of nuclear ETS1 did not change significantly in response to freezing, anoxia or dehydration (Figure 4). The DNA binding levels of total ETS1 and p-ETS1 (T38) showed similar trends. For total ETS1, the DNA binding activity increased by 1.57 \pm 0.12 -fold and by $1.44 \pm 0.085$-fold in response to freezing and dehydration respectively, but no changes was observed in response to anoxia treatment (Figure 5). The binding of p-ETS1 (T38) also increased by $2.2 \pm 0.26$-fold and $2.1 \pm 0.19$-fold in response to freezing and dehydration, respectively, while showing no significant change in response to anoxia exposure (Figure 5).

Comparison of ETS-1 structure

The primary sequences of ETS-1 from Xenopus tropicalis and humans were highly similar to each other. Therefore, we predicted that the 3-dimentional structures will also share high degree of similarity. Indeed, the predicted structure for Xenopus tropicalis (Figure 6A) and humans (Figure 6B) showed high degree of similarity when superimposed (Figure 6C), indicating that perhaps the two proteins will act similarly when bound to DNA (Figure 6D).

Relative expression of downstream targets 
To assess whether ETS1 is acting as a transcriptional activator or a transcriptional repressor, a list of targets containing the ETS1 binding domain within their promotor were curated using the ENCOCDE Transcription Factor Targets from Harmonizome (http://amp.pharm.mssm.edu/Harmonizome/). These targets were found to be regulated by ETS1 in multiple studies in both tissues and different cell lines. The first set of targets measured were the AOX1, PRX1 and PRX5 enzymes involved in ROS metabolism. Levels of AOX1 decreased in response to freezing (by $40 \pm 11 \%$ ) and dehydration (by $34 \pm 7.7 \%$ ) but remained unchanged after anoxia exposure (Figure 7). Levels of PRX1 increased by $1.9 \pm 0.23$-fold in response to freezing but showed no change in response to anoxia or dehydration treatments. Levels of PRX5 showed a decreasing trend in response to freezing and anoxia treatment but changes were not statistically significant; however, protein levels of PRX5 decreased by $54 \pm 3 \%$ in response to dehydration.

Levels of three proteins involved in the mitochondrial electron transport chain were also measured. Protein levels of ATP5A1 showed no change in response to freezing, anoxia or dehydration, whereas levels of NDUFAB1 decreased under all conditions (Figure 7). Protein levels of NDUFAB1 decreased by $70 \pm 3 \%$ in response to freezing, by $66 \pm 9 \%$ in response to anoxia, and by $72 \pm 4 \%$ in response to dehydration. Levels of CYC1 also decreased by $38 \pm 1.9 \%$ in response to freezing, showed no change in response to anoxia, but decreased by $40 \pm 7 \%$ in response to dehydration.

Levels of CS and GLUT1, two targets involved in glycolysis and glucose transport, respectively, were also measured. CS did not change in response to freezing or anoxia but showed a $53 \pm 7.4 \%$ decrease in response to dehydration (Figure 7 ). Levels of 
GLUT1 also decreased by $45 \pm 5.4 \%$ following freezing, by $65 \pm 7.2 \%$ in response to anoxia and by $57 \pm 19 \%$ in response to dehydration.

\subsection{Discussion}

Wood frogs are fascinating models for studying extreme stress tolerance in vertebrate species for they can independently tolerate anoxia, major dehydration and hyperglycemia, and in combination, have developed one of the most amazing vertebrate survival mechanisms - freeze tolerance [1]. High rates of glycogenolysis and glucose output from liver are detectable in wood frogs $<2$ min after the freezing exotherm is recorded, and concurrently, heart rate doubles to 8 beats/min as early as 1 minute postnucleation to facilitate glucose distribution as a cryoprotectant from the liver to all organs [38-40]. Elevated cardiac function persists for about $\sim 1$ hour post-nucleation and halts within $\sim 20 \mathrm{~h}$ as body ice content reaches its maximum [40]. Resumption of cardiac activity in early hours of thawing restores blood flow to other organs, and this is important for the wood frog's recovery because it has been hypothesized that resumption of tissue perfusion and the rise in energy demand by warming organs could potentially be the switch needed to change from anaerobic to aerobic metabolism [40]. Dehydration also elicits a hyperglycemic response where glucose levels increase to 1092, 1409 and $1263 \mathrm{nmol} / \mathrm{mg}$ of protein in brain, heart and liver of wood frog, respectively [3]. However, unlike freezing and dehydration, exposure to anoxia did not appear to initiate a hyperglycemic response in wood frogs probably due to the absence of mechanical stress on the cellular architecture [41].

Hyperglycemia and oxidative stress can result in drastic physiological and biochemical changes that lead to various outcomes in the cell. One of these is AGE 
formation that, under normal conditions, is a slow process and takes a significant amount of time (months up to years) before AGE accumulation results in irreversible damage. However, under conditions where hyperglycemia and oxidative stress co-occur, AGE formation is accelerated and levels can rise over only a few hours, thereby affecting even short-lived macromolecules [9]. Surprisingly, the present data showed that levels of AGE-adducts decreased significantly in response to freezing and, although they showed a decreasing trend in response to anoxia and dehydration, the changes observed were not statistically significant (Figure 2). Interestingly, in agreement with current results, a previous study demonstrated that levels of glycated hemoglobin in serum decreased in response to freezing [10]. The decrease in AGE-adduct levels during freezing could be due to the enhanced antioxidant response (both metabolites and detoxifying enzymes) that accompanies this condition. Antioxidant enzymes such as superoxide dismutase, glutathione peroxidase, catalase and carbonyl metabolizing enzymes such as aldo-keto reductases, aldehyde dehydrogenases, carbonyl reductases and glutathione S-transferases all participate in AGE clear-up from organs [42]. Indeed, freeze-tolerant wood frogs have higher basal antioxidant responses than non-freeze-tolerant frogs [7]. The same study reported that glutathione status in multiple tissues of wood frogs, including the heart, and lipid peroxidation as measured by thiobarbituric acid-reactive substances (TBARS) and by Fe(III)-xylenol orange complex formation also showed little to no change in multiple tissues in response to freezing [7]. This indicated that there was little to no increase in oxidative damage in wood frogs. Moreover, levels of glutathione peroxidase activity increased in all tissues in response to freezing, including the heart, which further indicates preparedness by wood frogs to deal with ROS generation [7]. In another study, 
levels of superoxide dismutase 2 increased in response to 4-hour freezing in wood frog heart but remained unchanged in response to 24-hour freezing, 24-hour anoxia or dehydration [43]. suggesting that perhaps that high basal levels of these antioxidant responses is sufficient to prevent excessive AGE-adduct formation. While freezing has been extensively studied regarding antioxidant defenses, such studies are lacking for anoxia or dehydration stressed wood frogs. Nonetheless, studies on other anoxia and dehydration tolerant frogs and turtles demonstrate an increase in antioxidant responses under these conditions [44-46]. As such, it is likely that wood frogs also elicit the same antioxidant responses to combat oxidative damage in response to anoxia and dehydration, which could result in lowering overall AGE levels.

HMGB1, normally a nuclear protein, can be post-translationally modified by acetylation, transported into the cytoplasm and eventually released into the extracellular matrix in response to oxidative stress or hyperglycemia [13,47]. The current results showed that total HMGB1 is significantly elevated in response to freezing, anoxia and dehydration (Figure 3) in wood frog heart. This elevation was paralleled by an increase in HMGB1 acetylation at K29 (Figure 3), which together suggests that, HMGB1 could potentially be translocated into the cytoplasm and subsequently into the extracellular matrix to bind to various cell surface receptors. A cDNA array analysis of gene expression by our group demonstrated an increase in RAGE transcript levels following freezing exposure in heart of wood frogs [16]. Congruent with these results, the current study reports an increase in RAGE protein levels in response to freezing, anoxia and dehydration (Figure 3), suggesting that perhaps these conditions favor the activation of RAGE signaling in wood frogs. Altogether, these results suggest that changes in either 
HMGB1 or AGE-adduct levels could be sufficient to bind to RAGE and initiate cascades of RAGE-dependent events that result in differential regulation of ETS1 transcriptional responses.

Binding of AGEs or HMGB1 to RAGE results in a cascade of events that lead to PKC $\beta I I$ activation by priming its phosphorylation and membrane anchoring, and leading to the activation of JNK and ERK1/2 kinases, respectively, under low oxygen conditions $[17,18,48]$. Activated ERK1/2 can then phosphorylate ETS1 to promote its nuclear translocation [19]. The present results showed no change in phosphorylation (activation) of PKC 3 II S660 in response to anoxia or freezing, but levels increased in response to dehydration (Figure 3). PKCs are responsible for regulating a large number of physiological changes such as cell polarity, proliferation and differentiation, migration and metabolism [49]; some of these are strictly attenuated under MRD. The trends seen for PKCßII S660 were similar to those for ERK2, where levels remained the same in response to freezing, slightly but significantly decreased in response to anoxia, and increased in response to dehydration (Figure 3). Signal transduction through PKCßII to ERK2 is mediated via JNK $[17,18]$. In response to freezing, JNK activity in the heart remained unchanged in wood frog [50], a result that correlates with the unchanged levels of active ERK2 (Figure 3). Moreover, while JNK activities have not been measured in anoxic wood frogs, studies on other anoxia tolerant models show an increase in JNK levels only in response to the early hours of anoxia exposure [51]. As such, perhaps the requirement for JNK-mediated activation of ERK2 is only needed in early stages of the stress and not when the animal is fully adapted 24-hour post-exposure. Altogether, although there is a lack of increase in ERK2 in response to freezing or anoxia, it is still 
likely that the basal level of ERK2 available can remain active and participate in ETS1 activation by phosphorylating this transcription factor.

The current study demonstrates no change in ETS1 levels in response to freezing and anoxia, but a small significant decrease in response to dehydration (Figure 3). ETS1 is an early response transcription factor that is induced or activated in response to the early stages of cellular stress, especially under condition of oxygen deprivation [52]. Regulating a remarkable range of signaling pathways, ETS1 is involved in many processes including, but not limited to, angiogenesis, apoptosis, energy metabolism, cellular energetics and autophagy [20,21,23,24]. As such, its timely expression and activation must be under strict regulation by various mechanisms to ensure that its transcriptional activities are stimulated appropriately. Given the involvement of ETS1 in many process, it is possible that at the time timepoints studies here, the regulation of ETS1 is not needed at the transcriptional level and that the amount of ETS1 available in cells is enough to carry out other processes as needed. The current study also investigated the phosphorylation status of ETS1 (T38) and results show that the phosphorylation level was not affected by freezing or anoxia but was significantly increased in response to dehydration (Figure 3). Posttranslational modifications are an energy-efficient way of regulating protein-enzyme activity, especially under conditions when ATP synthesis is attenuated. Indeed, numerous pathways and enzyme activities are regulated by reversible posttranslational modifications (PTMs) [34,53]. As shown in Table S1, ETS1 was predicted to have multiple phosphorylation sites, and was shown to undergo other forms of posttranslational modifications. However, due to limited commercial antibodies available that cross-react with wood frogs, we were unable to measure the levels of 
alternative modifications. Therefore, with respect to the data provided, it is possible that in addition to other modified forms, only basal levels of active p-ETS1 (T38) may be required under freezing and anoxic conditions, while more active ETS1 (T38) is needed to regulate gene expression during dehydration. An antibody that detects total ETS1 (both modified and unmodified) was used to assess the nuclear localization of this transcription factor. The results demonstrated that nuclear localization of ETS1 did not change significantly in response to freezing, anoxia or dehydration, although the trends were all decreasing (Figure 4). In response to a specific stimulus such as hypoxia, hyperglycemia, or oxidative stress, specific transcription factors are translocated to the nucleus to induce the expression of gene families required for mitigating the stress [52,54]. However, while some transcription factors are induced in response to stress, their downstream transcriptional activity may be temporally regulated. For example, freezing or dehydration could cause architectural damage to capillaries in an organ, but this is not always imminent. As such, maintaining a pool of transcription factors in the nucleus needed to deal with wound healing or capillary biogenesis is a pro-active measure to promote survival.

To determine whether the DNA binding activity of ETS1 in the heart is affected by stress in wood frogs, a DPI-ELISA was performed using antibodies that react with total ETS1 and one of its active forms, p-ETS1 (T38). Interestingly, whereas the nuclear localization of total ETS1 did not change in response to stress (Figure 4), the DNA binding by either ETS1 or p-ETS1 T38 increased in response to freezing and dehydration but was unchanged under anoxia (Figure 5). This suggests that in response to prolonged stress, the activity of ETS1 is regulated at the posttranslational level and that ETS1 
transcriptional activity plays a significant role in the cellular response to stress in wood frog heart. Not surprisingly, given the number of genes that this transcription factor regulates, and the hypometabolic consequences of the stresses imposed, using PTMs appears to be an effective and energy efficient way of controlling gene expression. The amphibian primary protein sequence (Figure S1) and its 3-dimentional structure (Figure 6) shares high degrees of similarity to that of human ETS1, which enabled us to use data from mammalian models to support our findings here in amphibians. Using the amphibian ETS1 as input, it was predicted that this transcription factor can be phosphorylated, SUMOylate and acetylated at multiple locations (Table S1). While it requires further validations, these predictions support our hypothesis that PTMs play a significant role in regulating ETS1 activity in the cell. Moreover, reports show that the DNA binding ability of ETS1 is enhanced in the presence of HMGB1 [22]. Therefore, the strong increase in total HMGB1 levels, as well as acetylated HMGB1, seen in this study (Figure 3) could support the enhanced DNA binding activity of p-ETS1 T38 in response to freezing or dehydration.

Several studies have reported that the ETS1 can act both as a transcriptional activator [22-24] and a transcriptional suppressor [23-26,30]. As such, based on available ChIP-seq information (see methods) and published data [23,24], a group of downstream targets known to be regulated by ETS1 were assembled and analyzed. The present results show that protein levels of AOX1 were significantly downregulated in response to freezing and dehydration (Figure 7). AOX1 is a cytosolic enzyme that plays an important role in xenobiotic metabolism, but has been shown recently to catalyzes the reduction of nitrites to $\mathrm{NO}$ in the presence of aldehyde containing substrates or NADH 
[55]. Interestingly, NO is known to regulate cardiac contractility and heart rate, and low levels of NO were shown to be protective against cardiac remodeling [56]. Whereas this could protect wood frog hearts during stress, it is possible that the activity of AOX1 is required in the early hours of stress exposure (pre-conditioning) or during the early hours of recovery (post-conditioning), and not when the heart is fully adapted following long term exposure to stress. In addition, oxidation of NADH by AOX1 can also result in the production of pro-oxidant species (ROS, RNS, superoxide anion radicals) that although necessary at low levels to initiate redox signaling, could be extremely pathogenic in high levels [57]. Therefore, given that frogs exposed to freezing, anoxia and dehydration are already at risk of oxidative stress, down-regulation of AOX1 could be a protective response in this situation. Interestingly, the DNA binding of both total and p-ETS1 T38 reflects the same trends as AOX1, indicating that ETS1 may be a transcriptional repressor of AOX1. In support of this argument, there are multiple ETS binding sites within the promotor region of the AOX1 gene $[23,24]$ and given its role in generating pro-oxidant species, it is possible that the transcriptional inhibition of AOX1 is mediated by ETS1. Further investigation is warranted to validate this hypothesis.

ETS1 has been also shown to regulate the expression of PRX1 and PRX5 [2224]. PRX1 is a typical 2-cysteine peroxidase localized to the cytoplasm, whereas PRX5 is an atypical 2-cysteine peroxidase that resides mainly in the cytoplasm and has a higher antioxidant activity in response to ROS [58]. Both of these peroxidases are stress responsive, and it was shown that their expression is mediated by ETS1 in response to hydrogen peroxide or hypoxia treatments [22]. Interestingly, the same study demonstrated that HMGB1 physically interact with ETS1 to enhance its transcriptional 
activity at PRX1 and PRX5 promotors. The current results show that expression of PRX1 was significantly regulated in response to freezing, whereas expression of PRX5 was significantly downregulated in response to dehydration (Figure 7). The DNA binding activity of ETS1 in response to freezing, correlates with an increase in the protein levels of PRX1 in response to the same treatment, therefore it is possible that this increase is mediated by ETS1. Moreover, PRX1 has been shown to play additional roles in cells by participating in cell signaling and chaperone activity. For example, in response to ionizing radiation, PRX1 was shown to interact with JNK to reduce its apoptotic activity, thereby promoting cell survival [59]. Moreover, in response to thermal stress, PRX1 has been shown to switch from being a peroxidase to acting as a protein chaperone that binds to denatured proteins [60]. In fact, the same study reported that PRX1 bound to CS in response to stress and prevented its denaturation and aggregation. As such, it is possible that the increase in PRX1 in response to freezing is not only a systemic response to combat AGE formation (as shown by decreasing levels during freezing) in the heart, but also acts to inhibit apoptosis or protein denaturation/aggregation during stress. Alternatively, although these antioxidant enzymes play roles in ROS detoxification, under conditions of MRD, the cell must strictly but strategically allocate its available energy to pro-survival processes only. Therefore, if other antioxidant enzymes with similar functions are up regulated, then inducing the expression of peroxiredoxins might not be necessary. Indeed, studies have reported that PRXs have overlapping detoxification activities with glutathione peroxidases and catalases. Importantly, the catalytic efficiency of PRXs $\left(\sim 10^{5} \mathrm{M}^{-1} \mathrm{~s}^{-1}\right)$ is substantially lower as compared to glutathione peroxidases $\left(10^{8} \mathrm{M}^{-1} \mathrm{~s}^{-1}\right)$ and catalases $\left(10^{6} \mathrm{M}^{-1} \mathrm{~s}^{-1}\right)[61,62]$. As such, it is 
possible that wood frogs reduce or maintain the basal expression of certain antioxidant enzymes in response to stress in favor of promoting the expression and activity of the more potent ones.

Overexpression of ETS1 has previously been shown to coincide with decreased expression of components of the mitochondrial electron transport chain including but not limited to NDUFAB1, CYC1 and ATP5A1 [23,24]. Mitochondria are the powerhouse of the cell and are central to energy metabolism under aerobic conditions. When oxygen levels are plentiful in cells, the mitochondria pump protons across the mitochondrial membrane; thereby generating a proton-motive force that allows $\mathrm{F}_{1} \mathrm{~F}_{0}$-ATPase to generate ATP from ADP [63]. However, under anerobic conditions the mitochondria become a liability as they become the major producers of ROS. In such circumstances, the $\mathrm{F}_{1} \mathrm{~F}_{0^{-}}$ ATPase commits "cellular treason" to maintain the proton-motive force by hydrolyzing ATP into ADP [63]; thereby limiting ATP availability in the cell when levels are already scarce. As such, animals that tolerate low oxygen conditions (hypoxia or anoxia) must limit ATP hydrolysis by either reducing the proton conductance of the inner mitochondrial membrane or inhibiting the activity of $\mathrm{F}_{1} \mathrm{~F}_{0}$-ATPase in order to survive [63]. A study on mitochondria isolated from the skeletal muscle of hypoxic frogs reported that due to decreased metabolic rates, the mitochondria exhibited a lower rate of proton leakage without affecting proton conductance [64]. Moreover, mitochondria isolated from anoxia tolerant frogs showed that although protein conductance was not altered in response to anoxia, the $\mathrm{F}_{1} \mathrm{~F}_{0}-\mathrm{ATPase}$ enzyme was greatly inhibited [63]. A decrease in $\mathrm{F}_{1} \mathrm{~F}_{0}$-ATPase activity was also reported in anoxic turtle brain, suggesting that this is an adaptive response [65]. In the present study, exposure to freezing, anoxia or dehydration 
did not affect the expression level of ATP5A1 (a component of the $\mathrm{F}_{1} \mathrm{~F}_{0}$-ATPase complex) (Figure 7), despite an increase in the DNA binding activity of ETS1 in response to freezing and dehydration (Figure 5). Given that the $\mathrm{F}_{1} \mathrm{~F}_{0}$-ATPase activity is hypothesized to be reduced in wood frogs under oxygen deprived conditions as an adaptive response, it is possible that $\mathrm{F}_{1} \mathrm{~F}_{0}$-ATPase inhibition occurs at another level or perhaps by PTMs under stress conditions. While possible, further investigation is warranted to validate this hypothesis.

The current study demonstrated a very strong reduction in levels of NDUFAB1 in response to freezing, anoxia and dehydration (Figure 7), which correlates with an increase in DNA binding of active ETS1 in freezing and dehydration but not anoxia (Figure 5). This protein plays a significant role in mitochondria by acting as an accessory protein for complex I-III, not only by stabilizing these complexes, but by promoting the synthesis of iron-sulfur centers needed in these subunits [27]. The importance of this protein in promoting cardio-protection against ischemia-reperfusion injury was attributed to its role in decreasing ROS production and stabilizing mitochondrial complexes [27]. While this is a protective response in stress intolerant animals, as discussed earlier, hypoxia/anoxia tolerant animals greatly reduce the activity of their mitochondria as an adaptive response to prevent disruption of the mitochondrial electrochemical gradient. As such, a reduction of NDUFAB1 levels in wood frogs under stress conditions, that may be mediated at the transcriptional level by ETS1-mediated promotor suppression, could contribute to the suppression of mitochondrial activity. Moreover, NDUFAB1 is also known as the mitochondrial acyl carrier protein and plays an essential role in mitochondria fatty acid synthesis [66]. This pathway is responsible for generating lipoic 
acid, a cofactor needed for the activity of several enzymes including but not limited to pyruvate dehydrogenase, oxoacid dehydrogenase, and $\alpha$-ketoglutarate dehydrogenase [66]. Whereas the activity of pyruvate dehydrogenase has not been studied in wood frog heart, freezing and anoxia exposure resulted in decreased pyruvate dehydrogenase complex activity in wood frog liver, an action that was linked to the efforts to attenuate glycolytic flux under these conditions, particularly when high rates of glycogenolysis must be directed into glucose synthesis and export as the cryoprotectant [34]. As such, a reduction in NDUFAB1 levels could play a role in suppressing the activity of the pyruvate dehydrogenase complex under stress conditions.

A reduction in $\mathrm{CYC} 1$ protein levels was also seen in response to freezing and dehydration (Figure 7) and correlates with the increase in DNA binding activity of ETS1 under the same conditions (Figure 5). This suggests that perhaps CYC1 transcription is inhibited by ETS1. In complex III (cytochrome bc1 complex) electrons are accepted from ubiquinol and are transferred to cytochrome $\mathrm{c}$, a process that also allows the translocation of protons across the inner mitochondrial membrane [67]. In this process, two electron molecules are sequentially transferred to $\mathrm{CYC1}$ via the Rieske iron-sulfur protein [67]. Theoretically, reduction or inhibition of the nuclear encoded mitochondrial genes could reduce ROS production under oxygen limiting conditions [67]. For example, low levels of cytochrome c would retain Rieske and CYC1 in their reduced forms, thereby preventing further oxidation of ubiquinol and subsequently the generation of ubisemiquinone, resulting in reduced ROS production at complex III. As such, the reduction in $\mathrm{CYCl}$ seen in this study is perhaps a regulatory mechanism by cells to attenuate ROS generation in wood frogs. This hypothesis is plausible since the electron 
transport chain has been reported to be in a more reduced form in response to stress in other hypoxia/anoxia tolerant organisms [63,64].

ETS1 also plays a role in metabolism by regulating a set of genes involved in glucose metabolism and uptake $[23,24]$. Interestingly, overexpression of ETS1 coincided with reduction in CS levels under hypoxic conditions, but silencing of ETS1 reduced levels of GLUT1 $[23,24,30]$. The current results show that levels of CS remained unchanged in response to freezing and anoxia but decreased significantly in response to dehydration (Figure 7). Although an increase in ETS1 DNA binding in response to freezing should have, in theory, decreased levels of CS as was seen in response to dehydration, it is possible that alternative regulatory mechanisms are involved in regulating CS activity or expression. Indeed, studies have shown a reduction in CS activity in response to anoxia in turtle brain [65], hypoxia in flour beetle [68], and hibernation in skeletal muscle of bears [69]; all of these reflect a lower oxidative capacity by the mitochondria. While the increase in active ETS1 DNA binding could potentially explain the regulation of CS at the transcriptional level in response to dehydration, it appears that CS protein levels and activity could be regulated at other stages downstream of its transcription. Indeed, reversible protein posttranslational modifications are an essential regulatory mechanism used to control enzymatic activity under hypometabolic states [53] but validation of such additional controls on CS is required. Lastly, levels of GLUT1 were greatly reduced in response to all three stresses in wood frog hearts (Figure 7), an outcome that also reflects active ETS1 DNA binding in response to freezing and dehydration (but not anoxia) (Figure 5). The importance of glucose as a cryoprotective and osmoprotective agent has been established in wood frogs in response to freezing and 
dehydration $[1,70]$. As such, it is expected that wood frogs induce the expression of glucose transporters in different tissues to promote the uptake of glucose. In fact, it has been shown that transcript levels of glut4, a transporter that is expressed in multiple tissues including the heart to accelerate glucose uptake, is significantly increased in response to anoxia in wood frog skeletal muscle [71]. Protein levels of GLUT2 also increase significantly in liver of wood frogs in response to freezing, hypoxia and glucose loading [72], suggesting that perhaps each tissue induces the expression of a certain GLUT in response to stress. Therefore, it is likely that levels of most or all GLUT isoforms are modulated under stress conditions to support the needs for glucose transport.

In conclusion, current results show that ETS1 is potentially activated by the HMGB1/RAGE axis. Moreover, enhanced DNA Binding activity by p-ETS1 T38 in response to freezing and dehydration could result in activating/suppressing a network of genes involved in the antioxidant response, mitochondrial energetics, and glucose metabolism. These adaptive responses appear to promote cytoprotection in wood frog heart that protects the tissue from freezing, anoxia, and dehydration stresses.

\section{Acknowledgements}

We thank J.M. Storey for editorial review of this manuscript. This study was supported by a Discovery Grant from the Natural Sciences and Engineering Research Council of Canada (Grant \#6793). K.B.S. holds the Canadian Research Chair in Molecular Physiology and R.A. holds an Ontario Graduate Scholarship. 


\subsection{References- Chapter 4}

[1] K.B. Storey, J.M. Storey, Molecular physiology of freeze tolerance in vertebrates, Physiol. Rev. 97 (2017) 623-665. https://doi.org/10.1152/physrev.00016.2016.

[2] K.B. Storey, J.M. Storey, Freeze tolerance in animals, Physiol. Rev. 68 (1988) $27-$ 84. https://doi.org/10.1152/physrev.1988.68.1.27.

[3] T.A. Churchill, K.B. Storey, Dehydration tolerance in wood frogs: A new perspective on development of amphibian freeze tolerance, Am. J. Physiol. Integr. Comp. Physiol. 265 (1993) R1324-R1332.

https://doi.org/10.1152/ajpregu.1993.265.6.R1324.

[4] C.P. Holden, K.B. Storey, Second messenger and cAMP-dependent protein kinase responses to dehydration and anoxia stresses in frogs., J. Comp. Physiol. B. 167 (1997) 305-12. https://doi.org/10.1007/s003600050078.

[5] V.E.M. Gerber, S. Wijenayake, K.B. Storey, Anti-apoptotic response during anoxia and recovery in a freeze-tolerant wood frog (Rana sylvatica)., PeerJ. 4 (2016) e1834. https://doi.org/10.7717/peerj.1834.

[6] J.M. Storey, K.B. Storey, In defense of proteins: Chaperones respond to freezing, anoxia, or dehydration stress in tissues of freeze tolerant wood frogs, J. Exp. Zool. Part A Ecol. Integr. Physiol. 331 (2019) 392-402. https://doi.org/10.1002/jez.2306.

[7] D.R. Joanisse, K.B. Storey, Oxidative damage and antioxidants in Rana sylvatica, the freeze-tolerant wood frog., Am. J. Physiol. 271 (1996) R545-53. https://doi.org/10.1152/ajpregu.1996.271.3.R545.

[8] T.J. Lyons, A.J. Jenkins, Glycation, oxidation, and lipoxidation in the development of the complications of diabetes: A carbonyl stress hypothesis., Diabetes Rev. (Alexandria, Va.). 5 (1997) 365-391.

[9] A.W. Stitt, J.E. Moore, J.A. Sharkey, G. Murphy, D.A.C. Simpson, R. Bucala, H. Vlassara, D.B. Archer, Advanced glycation end products in vitreous: Structural and functional implications for diabetic vitreopathy., Invest. Ophthalmol. Vis. Sci. 39 (1998) 2517-23. http://www.ncbi.nlm.nih.gov/pubmed/9856760.

[10] J.A. MacDonald, T. Degenhardt, J.W. Baynes, K.B. Storey, Glycation of wood frog (Rana sylvatica) hemoglobin and blood proteins: In vivo and in vitro studies, Cryobiology. 59 (2009) 223-225. https://doi.org/10.1016/j.cryobiol.2009.06.008.

[11] A. Bierhaus, P.M. Humpert, M. Morcos, T. Wendt, T. Chavakis, B. Arnold, D.M. Stern, P.P. Nawroth, Understanding RAGE, the receptor for advanced glycation end products, J. Mol. Med. 83 (2005) 876-886. https://doi.org/10.1007/s00109005-0688-7.

[12] H. Yang, H. Wang, S.S. Chavan, U. Andersson, High mobility group box protein 1 (HMGB1): The prototypical endogenous danger molecule, Mol. Med. 21 (2015) S6-S12. https://doi.org/10.2119/molmed.2015.00087. 
[13] B. Lu, D.J. Antoine, K. Kwan, P. Lundback, H. Wahamaa, H. Schierbeck, M. Robinson, M.A.D. Van Zoelen, H. Yang, J. Li, H. Erlandsson-Harris, S.S. Chavan, H. Wang, U. Andersson, K.J. Tracey, JAK/STAT1 signaling promotes HMGB1 hyperacetylation and nuclear translocation, Proc. Natl. Acad. Sci. 111 (2014) 3068-3073. https://doi.org/10.1073/pnas.1316925111.

[14] J.S. Park, F. Gamboni-Robertson, Q. He, D. Svetkauskaite, J.-Y. Kim, D. Strassheim, J.-W. Sohn, S. Yamada, I. Maruyama, A. Banerjee, A. Ishizaka, E. Abraham, High mobility group box 1 protein interacts with multiple Toll-like receptors., Am. J. Physiol. Cell Physiol. 290 (2006) C917-24. https://doi.org/10.1152/ajpcell.00401.2005.

[15] C.-Z. Wu, J.-J. Zheng, Y.-H. Bai, P. Xia, H.-C. Zhang, Y. Guo, HMGB1/RAGE axis mediates the apoptosis, invasion, autophagy, and angiogenesis of the renal cell carcinoma., Onco. Targets. Ther. 11 (2018) 4501-4510. https://doi.org/10.2147/OTT.S167197.

[16] K.B. Storey, Strategies for exploration of freeze responsive gene expression: Advances in vertebrate freeze tolerance, Cryobiology. 48 (2004) 134-145. https://doi.org/10.1016/j.cryobiol.2003.10.008.

[17] Y. Xu, F. Toure, W. Qu, L. Lin, F. Song, X. Shen, R. Rosario, J. Garcia, A.M. Schmidt, S.F. Yan, Advanced glycation end product (AGE)-receptor for age (RAGE) signaling and up-regulation of Egr-1 in hypoxic macrophages, J. Biol. Chem. 285 (2010) 23233-23240. https://doi.org/10.1074/jbc.M110.117457.

[18] J.S. Chang, T. Wendt, W. Qu, L. Kong, Y.S. Zou, A.M. Schmidt, S.F. Yan, Oxygen deprivation triggers upregulation of early growth response-1 by the receptor for advanced glycation end products, Circ. Res. 102 (2008) 905-913. https://doi.org/10.1161/CIRCRESAHA.107.165308.

[19] W.K. Wang, Q.H. Lu, J.N. Zhang, B. Wang, X.J. Liu, F.S. An, W.D. Qin, X.Y. Chen, W.Q. Dong, C. Zhang, Y. Zhang, M.X. Zhang, HMGB1 mediates hyperglycaemia-induced cardiomyocyte apoptosis via ERK/Ets-1 signalling pathway, J. Cell. Mol. Med. 18 (2014) 2311-2320.

https://doi.org/10.1111/jcmm.12399.

[20] Z. Gao, G.H. Kim, A.C. Mackinnon, A.E. Flagg, B. Bassett, J.U. Earley, E.C. Svensson, Ets1 is required for proper migration and differentiation of the cardiac neural crest., Development. 137 (2010) 1543-51. https://doi.org/10.1242/dev.047696.

[21] H. Pei, C. Li, Y. Adereth, T. Hsu, D.K. Watson, R. Li, Caspase-1 is a direct target gene of ETS1 and plays a role in ETS1-induced apoptosis, Cancer Res. 65 (2005) 7205-7213. https://doi.org/10.1158/0008-5472.CAN-04-3566.

[22] M. Shiota, H. Izumi, N. Miyamoto, T. Onitsuka, E. Kashiwagi, A. Kidani, G. Hirano, M. Takahashi, M. Ono, M. Kuwano, S. Naito, Y. Sasaguri, K. Kohno, Ets regulates peroxiredoxin 1 and 5 expressions through their interaction with the highmobility group protein B1, Cancer Sci. 99 (2008) 1950-1959. 
https://doi.org/10.1111/j.1349-7006.2008.00912.x.

[23] M.L. Verschoor, L.A. Wilson, C.P. Verschoor, G. Singh, Ets-1 Regulates Energy Metabolism in Cancer Cells, PLoS One. 5 (2010) e13565. https://doi.org/10.1371/journal.pone.0013565.

[24] M.L. Verschoor, C.P. Verschoor, G. Singh, Ets-1 global gene expression profile reveals associations with metabolism and oxidative stress in ovarian and breast cancers, Cancer Metab. 1 (2013) 17. https://doi.org/10.1186/2049-3002-1-17.

[25] G. Mavrothalassitis, J. Ghysdael, Proteins of the ETS family with transcriptional repressor activity, Oncogene. 19 (2000) 6524-6532. https://doi.org/10.1038/sj.onc.1204045.

[26] D. Goldman, M.K. Sapru, S. Stewart, J. Plotkin, T.A. Libermann, B. Wasylyk, K. Guan, Cloning and characterization of GETS-1, a goldfish Ets family member that functions as a transcriptional repressor in muscle, Biochem. J. 335 (1998) 267275. https://doi.org/10.1042/bj3350267.

[27] T. Hou, R. Zhang, C. Jian, W. Ding, Y. Wang, S. Ling, Q. Ma, X. Hu, H. Cheng, $\mathrm{X}$. Wang, NDUFAB1 confers cardio-protection by enhancing mitochondrial bioenergetics through coordination of respiratory complex and supercomplex assembly, Cell Res. 29 (2019) 754-766. https://doi.org/10.1038/s41422-019-0208$\mathrm{x}$.

[28] R. Zhang, T. Hou, H. Cheng, X. Wang, NDUFAB1 protects against obesity and insulin resistance by enhancing mitochondrial metabolism, FASEB J. 33 (2019) 13310-13322. https://doi.org/10.1096/fj.201901117RR.

[29] R. Ni, D. Zheng, S. Xiong, D.J. Hill, T. Sun, R.B. Gardiner, G.-C. Fan, Y. Lu, E.D. Abel, P.A. Greer, T. Peng, Mitochondrial calpain-1 disrupts ATP synthase and induces superoxide generation in type 1 diabetic hearts: A novel mechanism contributing to diabetic cardiomyopathy., Diabetes. 65 (2016) 255-68. https://doi.org/10.2337/db15-0963.

[30] X. Zhang, D. Wu, M. Aldarouish, X. Yin, C. Li, C. Wang, ETS-1: A potential target of glycolysis for metabolic therapy by regulating glucose metabolism in pancreatic cancer, Int. J. Oncol. 50 (2017) 232-240. https://doi.org/10.3892/ijo.2016.3770.

[31] D. Shao, R. Tian, Glucose transporters in cardiac metabolism and hypertrophy, in: Compr. Physiol., John Wiley \& Sons, Inc., Hoboken, NJ, USA, 2015: pp. 331351. https://doi.org/10.1002/cphy.c150016.

[32] Y. Zhang, R. Al-attar, K.B. Storey, TonEBP/NFAT5 regulates downstream osmoregulatory proteins during freeze-thaw stress in the wood frog, Cryobiology. 79 (2017) 43-49. https://doi.org/10.1016/j.cryobiol.2017.09.003.

[33] R. Al-attar, Y. Zhang, K.B. Storey, Osmolyte regulation by TonEBP/NFAT5 during anoxia-recovery and dehydration-rehydration stresses in the freeze-tolerant wood frog (Rana sylvatica), PeerJ. 5 (2017) e2797. 
https://doi.org/10.7717/peerj.2797.

[34] R. Al-Attar, S. Wijenayake, K.B. Storey, Metabolic reorganization in winter: Regulation of pyruvate dehydrogenase (PDH) during long-term freezing and anoxia., Cryobiology. 86 (2019) 10-18. https://doi.org/10.1016/j.cryobiol.2019.01.006.

[35] R. Al-Attar, K.B. Storey, Effects of anoxic exposure on the nuclear factor of activated T cell (NFAT) transcription factors in the stress-tolerant wood frog., Cell Biochem. Funct. 36 (2018) 420-430. https://doi.org/10.1002/cbf.3362.

[36] S.L. Eaton, S.L. Roche, M. Llavero Hurtado, K.J. Oldknow, C. Farquharson, T.H. Gillingwater, T.M. Wishart, Total protein analysis as a reliable loading control for quantitative fluorescent western blotting, PLoS One. 8 (2013) e72457. https://doi.org/10.1371/journal.pone.0072457.

[37] J. Zhang, K.B. Storey, RBioplot: An easy-to-use R pipeline for automated statistical analysis and data visualization in molecular biology and biochemistry, PeerJ. 4 (2016) e2436. https://doi.org/10.7717/peerj.2436.

[38] J.M. Storey, K.B. Storey, $\beta$-Adrenergic, hormonal, and nervous influences on cryoprotectant synthesis by liver of the freeze-tolerant wood frog Rana sylvatica, Cryobiology. 33 (1996) 186-195. https://doi.org/10.1006/cryo.1996.0019.

[39] K.B. Storey, J.M. Storey, Biochemical adaption for freezing tolerance in the wood frog, Rana sylvatica, J. Comp. Physiol. B. 155 (1984) 29-36. https://doi.org/10.1007/BF00688788.

[40] J.R. Layne, R.E. Lee, T.L. Heil, Freezing-induced changes in the heart rate of wood frogs (Rana sylvatica)., Am. J. Physiol. 257 (1989) R1046-9. https://doi.org/10.1152/ajpregu.1989.257.5.R1046.

[41] C.P. Holden, K.B. Storey, Signal transduction, second messenger, and protein kinase responses during freezing exposures in wood frogs, Am. J. Physiol. Integr. Comp. Physiol. 271 (1996) R1205-R1211. https://doi.org/10.1152/ajpregu.1996.271.5.R1205.

[42] E.M. Ellis, Reactive carbonyls and oxidative stress: Potential for therapeutic intervention, Pharmacol. Ther. 115 (2007) 13-24. https://doi.org/10.1016/j.pharmthera.2007.03.015.

[43] C.-W. Wu, S.N. Tessier, K.B. Storey, Stress-induced antioxidant defense and protein chaperone response in the freeze-tolerant wood frog Rana sylvatica, Cell Stress Chaperones. 23 (2018) 1205-1217. https://doi.org/10.1007/s12192-0180926-x.

[44] M. Hermes-Lima, K.B. Storey, Relationship between anoxia exposure and antioxidant status in the frog Rana pipiens, Am. J. Physiol. Integr. Comp. Physiol. 271 (1996) R918-R925. https://doi.org/10.1152/ajpregu.1996.271.4.R918.

[45] A. Bundgaard, A.M. James, W. Joyce, M.P. Murphy, A. Fago, Suppression of reactive oxygen species generation in heart mitochondria from anoxic turtles: The 
role of complex I S -nitrosation, J. Exp. Biol. 221 (2018) jeb174391. https://doi.org/10.1242/jeb.174391.

[46] A.I. Malik, K.B. Storey, Activation of antioxidant defense during dehydration stress in the African clawed frog, Gene. 442 (2009) 99-107. https://doi.org/10.1016/j.gene.2009.04.007.

[47] D. Yao, M. Brownlee, Hyperglycemia-induced reactive oxygen species increase expression of the receptor for advanced glycation end products (RAGE) and RAGE ligands, Diabetes. 59 (2010) 249-255. https://doi.org/db09-0801 [pii];10.2337/db09-0801 [doi].

[48] T.A. Leonard, B. Różycki, L.F. Saidi, G. Hummer, J.H. Hurley, Crystal structure and allosteric activation of protein kinase C BII, Cell. 144 (2011) 55-66. https://doi.org/10.1016/j.cell.2010.12.013.

[49] C. Rosse, M. Linch, S. Kermorgant, A.J.M. Cameron, K. Boeckeler, P.J. Parker, PKC and the control of localized signal dynamics, Nat. Rev. Mol. Cell Biol. 11 (2010) 103-112. https://doi.org/10.1038/nrm2847.

[50] S.C. Greenway, K.B. Storey, Activation of mitogen-activated protein kinases during natural freezing and thawing in the wood frog, Mol. Cell. Biochem. 209 (2000) 29-37. https://doi.org/10.1023/a:1007077522680.

[51] S.C. Greenway, K.B. Storey, Discordant responses of mitogen-activated protein kinases to anoxia and freezing exposures in hatchling turtles, J. Comp. Physiol. B Biochem. Syst. Environ. Physiol. 169 (1999) 521-527. https://doi.org/10.1007/s003600050251.

[52] N. Qiao, C. Xu, Y.-X. Zhu, Y. Cao, D.-C. Liu, X. Han, Ets-1 as an early response gene against hypoxia-induced apoptosis in pancreatic $\beta$-cells., Cell Death Dis. 6 (2015) e1650. https://doi.org/10.1038/cddis.2015.8.

[53] R. Al-Attar, K.B. Storey, Suspended in time: Molecular responses to hibernation also promote longevity., Exp. Gerontol. 134 (2020) 110889. https://doi.org/10.1016/j.exger.2020.110889.

[54] S.F. Yan, J. Lu, Y.S. Zou, J. Soh-Won, D.M. Cohen, P.M. Buttrick, D.R. Cooper, S.F. Steinberg, N. Mackman, D.J. Pinsky, D.M. Stern, Hypoxia-associated induction of early growth response-1 gene expression, J. Biol. Chem. 274 (1999) 15030-15040. https://doi.org/10.1074/jbc.274.21.15030.

[55] H. Li, T.K. Kundu, J.L. Zweier, Characterization of the magnitude and mechanism of aldehyde oxidase-mediated nitric oxide production from nitrite, J. Biol. Chem. 284 (2009) 33850-33858. https://doi.org/10.1074/jbc.M109.019125.

[56] R. Rastaldo, P. Pagliaro, S. Cappello, C. Penna, D. Mancardi, N. Westerhof, G. Losano, Nitric oxide and cardiac function, Life Sci. 81 (2007) 779-793. https://doi.org/10.1016/j.lfs.2007.07.019.

[57] T.K. Kundu, M. Velayutham, J.L. Zweier, Aldehyde oxidase functions as a superoxide generating NADH oxidase: An important redox regulated pathway of 
cellular oxygen radical formation, Biochemistry. 51 (2012) 2930-2939. https://doi.org/10.1021/bi3000879.

[58] A. Perkins, K.J. Nelson, D. Parsonage, L.B. Poole, P.A. Karplus, Peroxiredoxins: Guardians against oxidative stress and modulators of peroxide signaling, Trends Biochem. Sci. 40 (2015) 435-445. https://doi.org/10.1016/j.tibs.2015.05.001.

[59] Y.-J. Kim, W.-S. Lee, C. Ip, H.-Z. Chae, E.-M. Park, Y.-M. Park, Prx1 suppresses radiation-induced c-Jun NH 2 -rerminal kinase signaling in lung cancer cells through interaction with the glutathione $\mathrm{S}$-transferase $\mathrm{Pi} / \mathrm{c}-\mathrm{Jun} \mathrm{NH} 2$-terminal kinase complex, Cancer Res. 66 (2006) 7136-7142. https://doi.org/10.1158/00085472.CAN-05-4446.

[60] I. Hanukoglu, R. Rapoport, Routes and regulation of NADPH production in steroidogenic mitochondria, Endocr Res. 21 (1995) 231-241.

[61] B. Hofmann, H.-J. Hecht, L. Flohé, Peroxiredoxins, Biol. Chem. 383 (2002) 34764. https://doi.org/10.1515/BC.2002.040.

[62] A. Hillar, B. Peters, R. Pauls, A. Loboda, H. Zhang, A.G. Mauk, P.C. Loewen, Modulation of the activities of catalase-peroxidase HPI of Escherichia coli by sitedirected mutagenesis $\dagger$, Biochemistry. 39 (2000) 5868-5875. https://doi.org/10.1021/bi0000059.

[63] J. St-Pierre, M.D. Brand, R.G. Boutilier, Mitochondria as ATP consumers: Cellular treason in anoxia, Proc. Natl. Acad. Sci. 97 (2000) 8670-8674. https://doi.org/10.1073/pnas.140093597.

[64] J. St-Pierre, M.D. Brand, R.G. Boutilier, The effect of metabolic depression on proton leak rate in mitochondria from hibernating frogs, J. Exp. Biol. 203 (2000) $1469-1476$.

[65] M.E. Pamenter, C.R. Gomez, J.G. Richards, W.K. Milsom, Mitochondrial responses to prolonged anoxia in brain of red-eared slider turtles, Biol. Lett. 12 (2016) 20150797. https://doi.org/10.1098/rsbl.2015.0797.

[66] S. Brody, C. Oh, U. Hoja, E. Schweizer, Mitochondrial acyl carrier protein is involved in lipoic acid synthesis in Saccharomyces cerevisiae, FEBS Lett. 408 (1997) 217-220. https://doi.org/10.1016/S0014-5793(97)00428-6.

[67] R.D. Guzy, P.T. Schumacker, Oxygen sensing by mitochondria at complex III: The paradox of increased reactive oxygen species during hypoxia, Exp. Physiol. 91 (2006) 807-819. https://doi.org/10.1113/expphysiol.2006.033506.

[68] L. Wang, S. Cui, Z. Liu, Y. Ping, J. Qiu, X. Geng, Inhibition of mitochondrial respiration under hypoxia and increased antioxidant activity after reoxygenation of Tribolium castaneum, PLoS One. 13 (2018) e0199056. https://doi.org/10.1371/journal.pone.0199056.

[69] B. Chazarin, K.B. Storey, A. Ziemianin, S. Chanon, M. Plumel, I. Chery, C. Durand, A.L. Evans, J.M. Arnemo, A. Zedrosser, J.E. Swenson, G. GauquelinKoch, C. Simon, S. Blanc, E. Lefai, F. Bertile, Metabolic reprogramming 
involving glycolysis in the hibernating brown bear skeletal muscle, Front. Zool. 16 (2019) 12. https://doi.org/10.1186/s12983-019-0312-2.

[70] T.A. Churchill, K.B. Storey, Metabolic responses to dehydration by liver of the wood frog, Rana sylvatica, Can. J. Zool. 72 (1994) 1420-1425. https://doi.org/10.1139/z94-188.

[71] M.P. Hoyeck, H. Hadj-Moussa, K.B. Storey, The role of MEF2 transcription factors in dehydration and anoxia survival in Rana sylvatica skeletal muscle, PeerJ. 5 (2017) e4014. https://doi.org/10.7717/peerj.4014.

[72] A.J. Rosendale, R.E. Lee, J.P. Costanzo, Effect of physiological stress on expression of glucose transporter 2 in liver of the wood frog, Rana sylvatica, $\mathrm{J}$. Exp. Zool. Part A Ecol. Genet. Physiol. 321 (2014) 566-576.

https://doi.org/10.1002/jez.1885. 


\section{Figure}

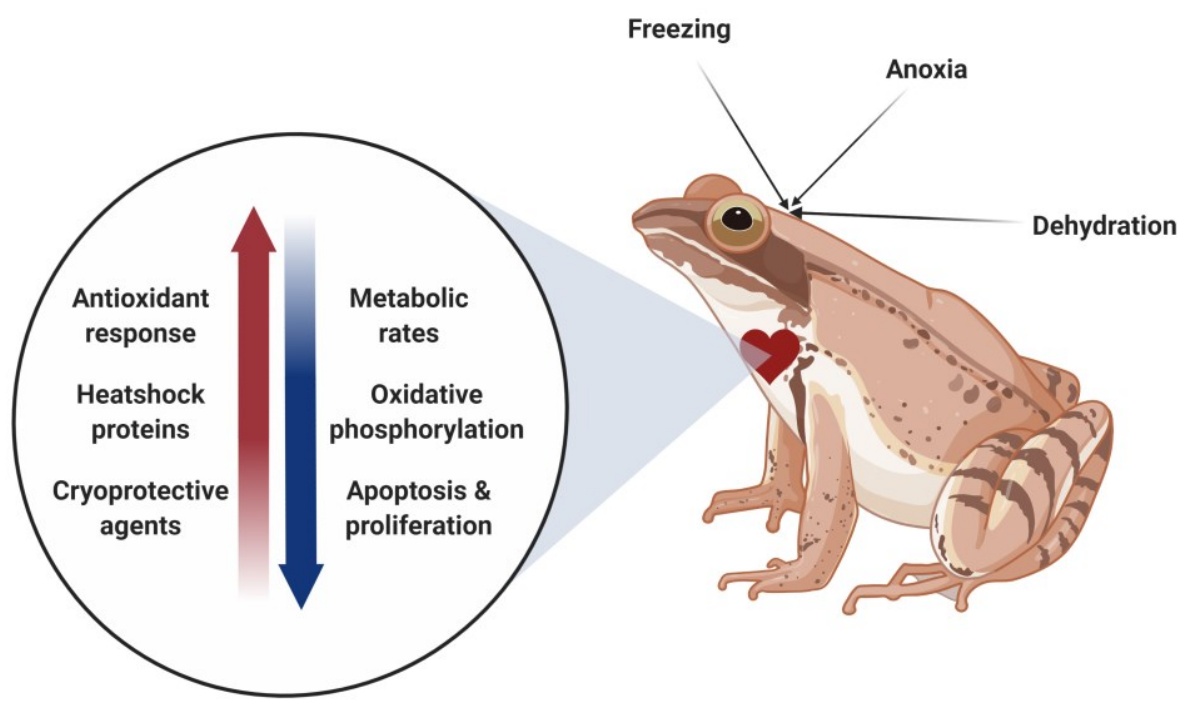

Figure 4.1: Schematic diagram representing physiological and molecular responses that occur during metabolic rate depression in wood frogs. Illustration was created by BioRender (https://biorender.com/). 


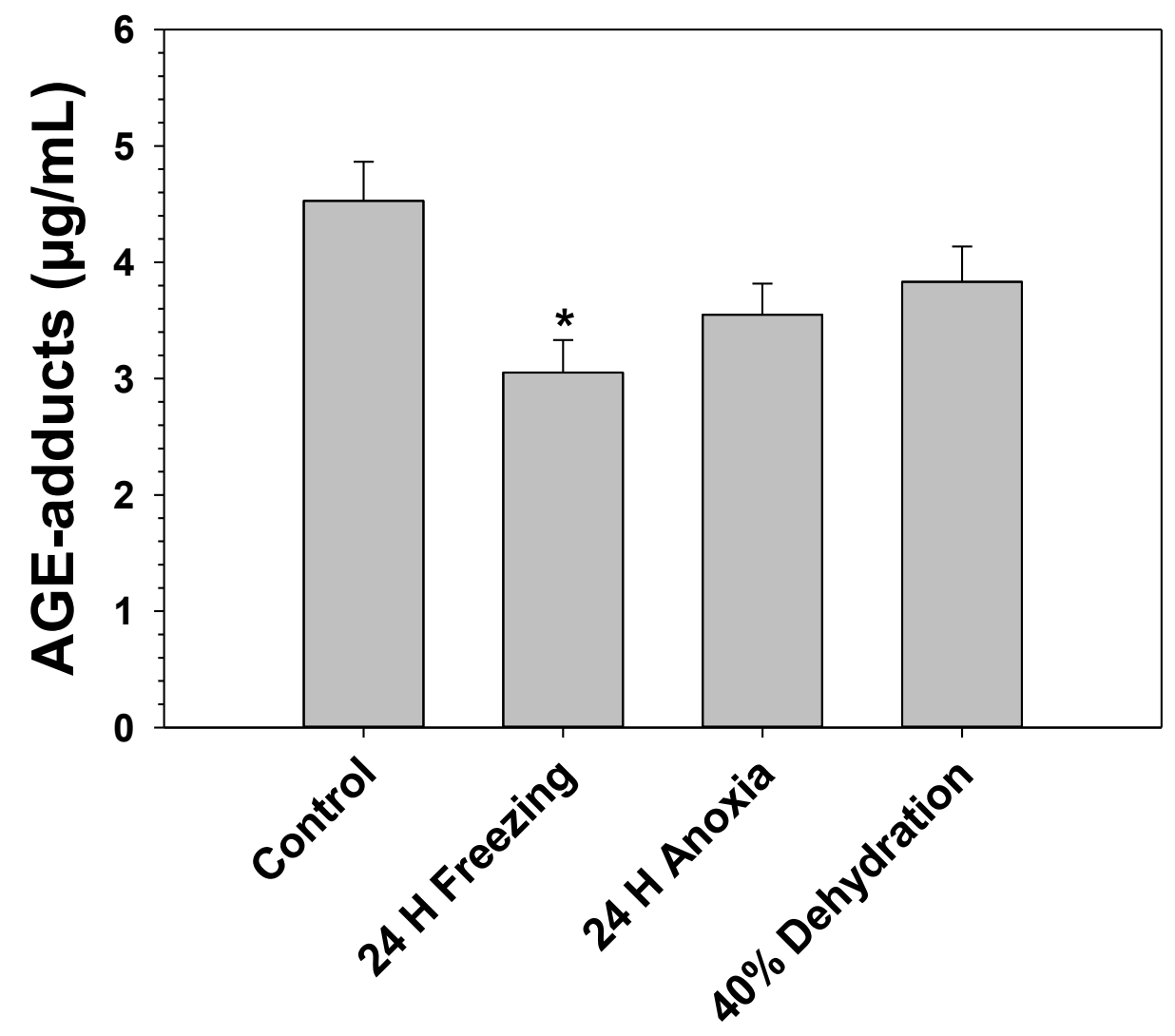

Figure 4.2: Levels of AGE-adducts in wood frog heart in response to freezing, anoxia or dehydration measured using a commercially available assay. Histogram shows mean \pm SEM of $\mathrm{n}=4$ independent biological replicates from different frogs. Statistical significance from the control is denoted by $\left(^{*}\right)(\mathrm{P}<0.05)$ and was calculated using a oneway ANOVA with a Dunnett post-hoc test. 

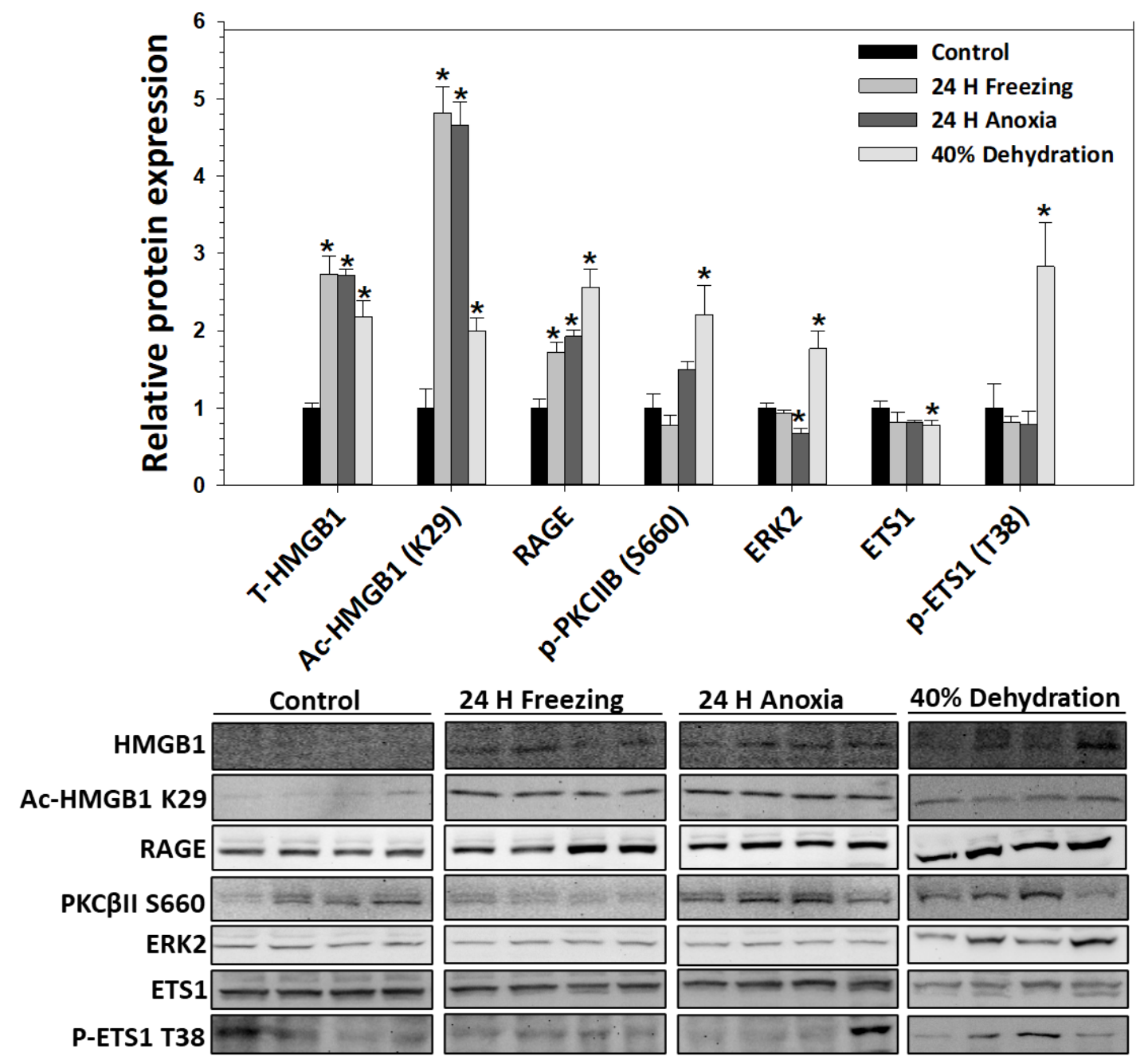

Figure 4.3: Protein levels of upstream RAGE activators and downstream RAGEregulated targets measured using western blotting. For more information, please see Figure 2. 


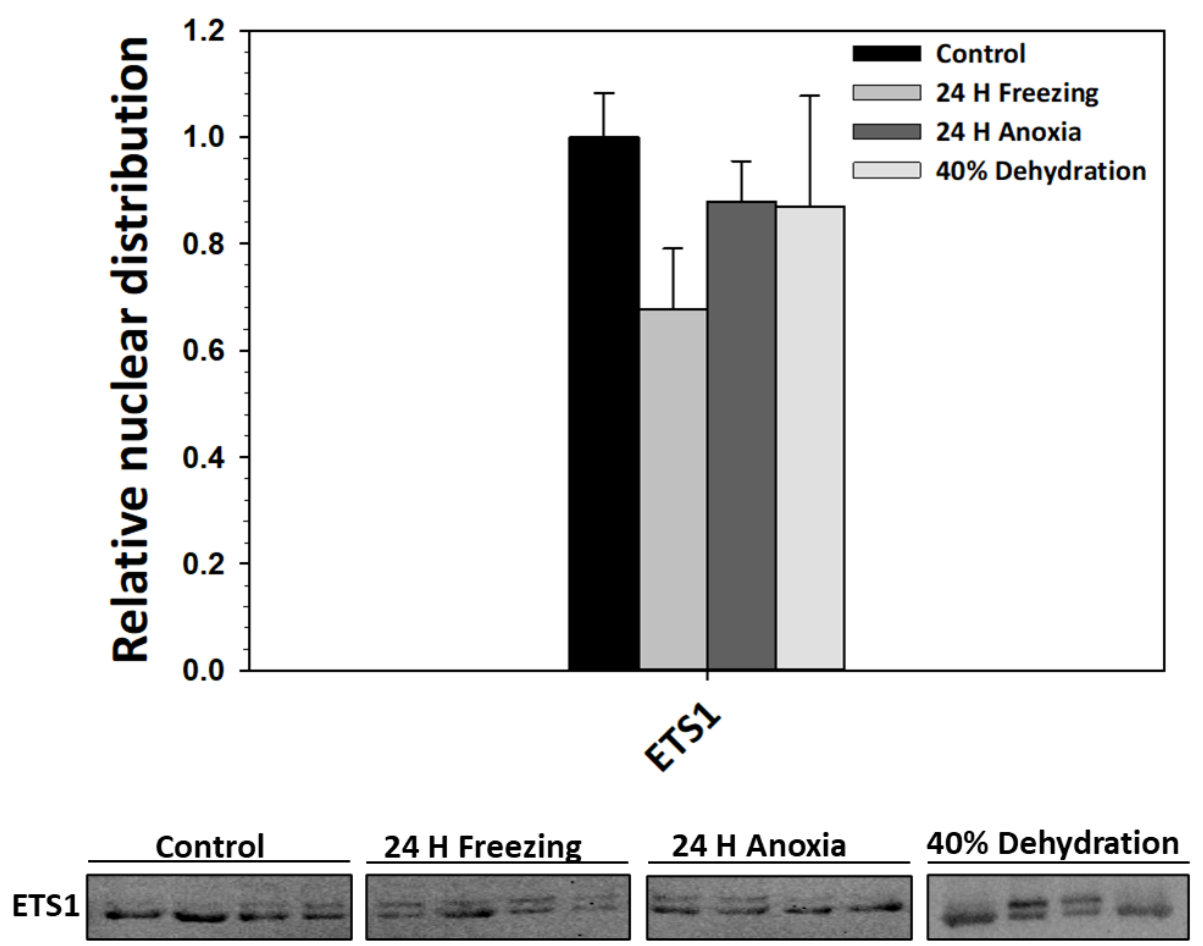

Figure 4.4: Relative nuclear localization of ETS1 proteins in response to freezing, anoxia or dehydration measured by western blotting in wood frog heart. For more information, see Figure 4.2. 


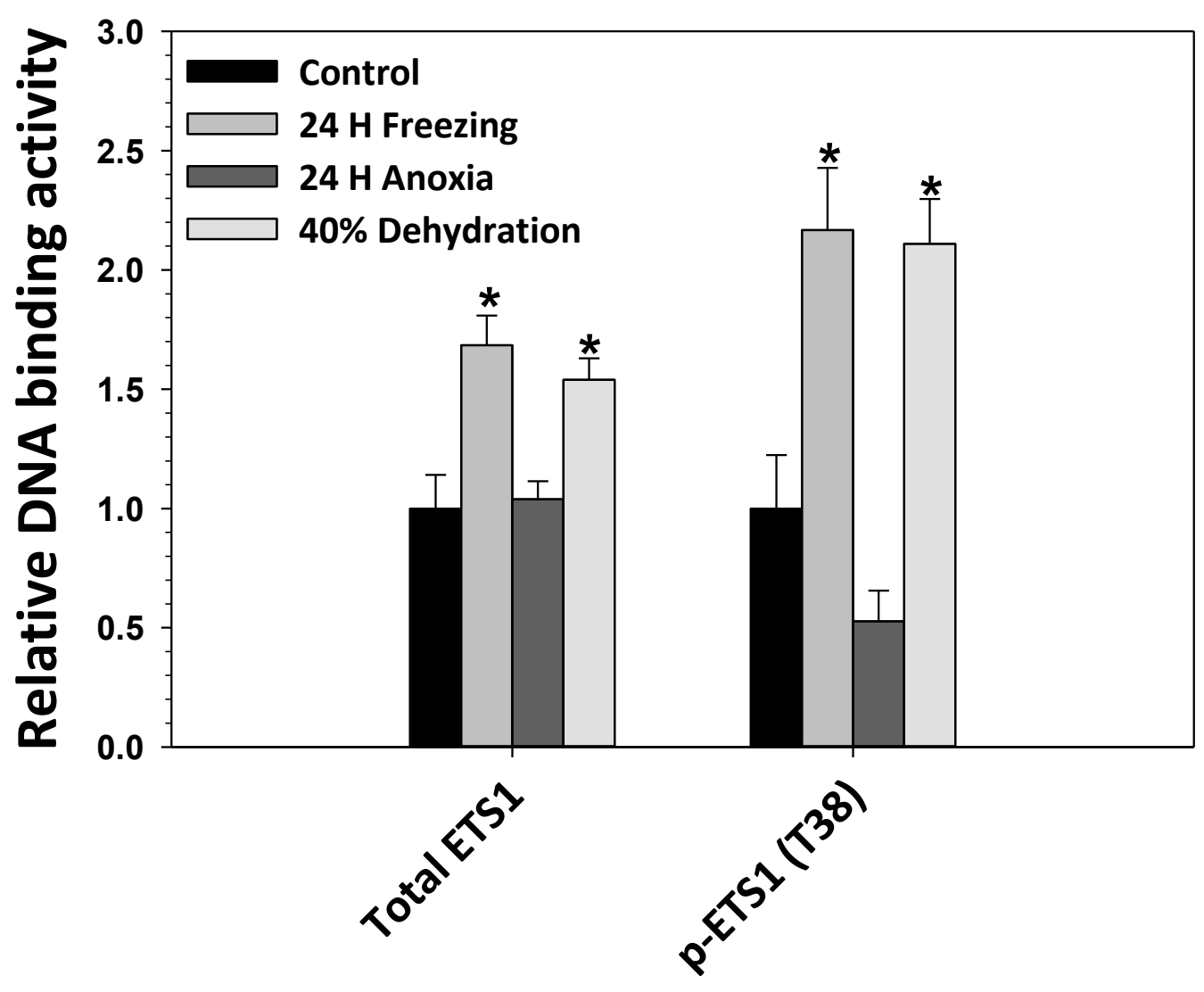

Figure 4.5: Relative DNA binding activity of p-ETS1 (T38) in response to freezing, anoxia or dehydration in wood frog heart. For more information, see Figure 4.2. 


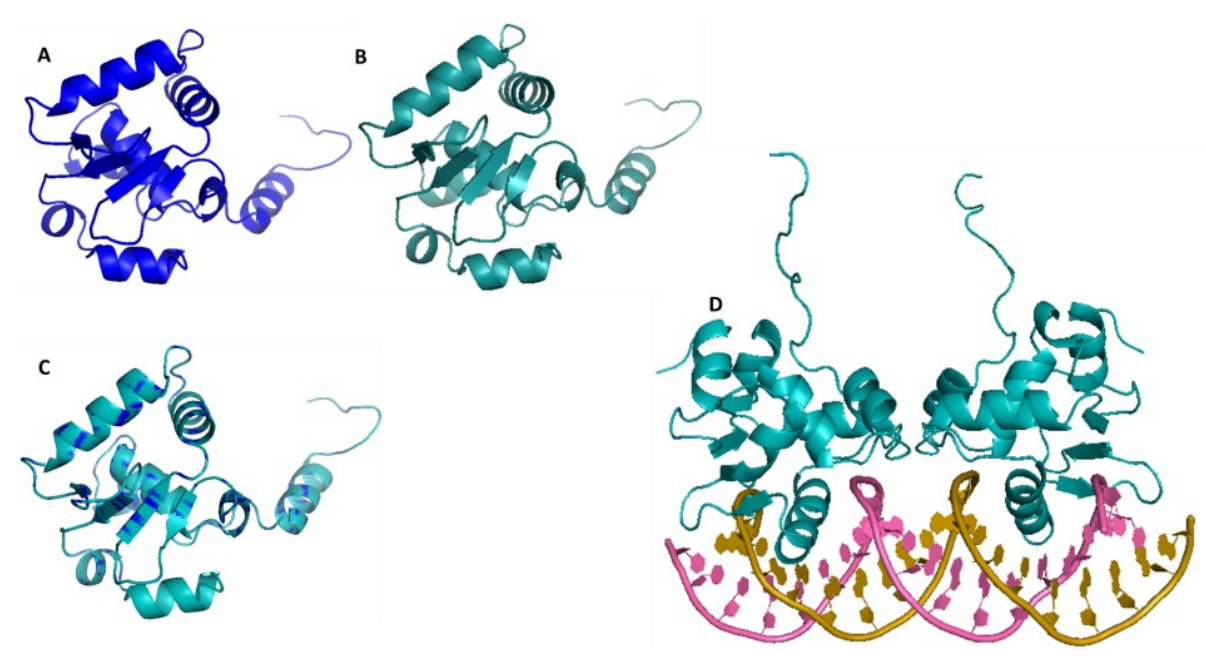

Figure 4.6: Schematic diagram showing the similarities between the ETS1 monomer generated using the protein sequence of Xenopus tropicalis (NP_001123840_1) and Humans (P14921.1). Using the online Phyre2 program, the ETS1 structure for Xenopus tropicalis (A) and human (B) were predicted. Using Pymol, both figures were superimposed (C) to show conservancy between the two structures. D) The Crystal structure of ETS1 homodimer DNA complex was retrieved from the PDB protein data bank (2nny), manipulated using Pymol. 

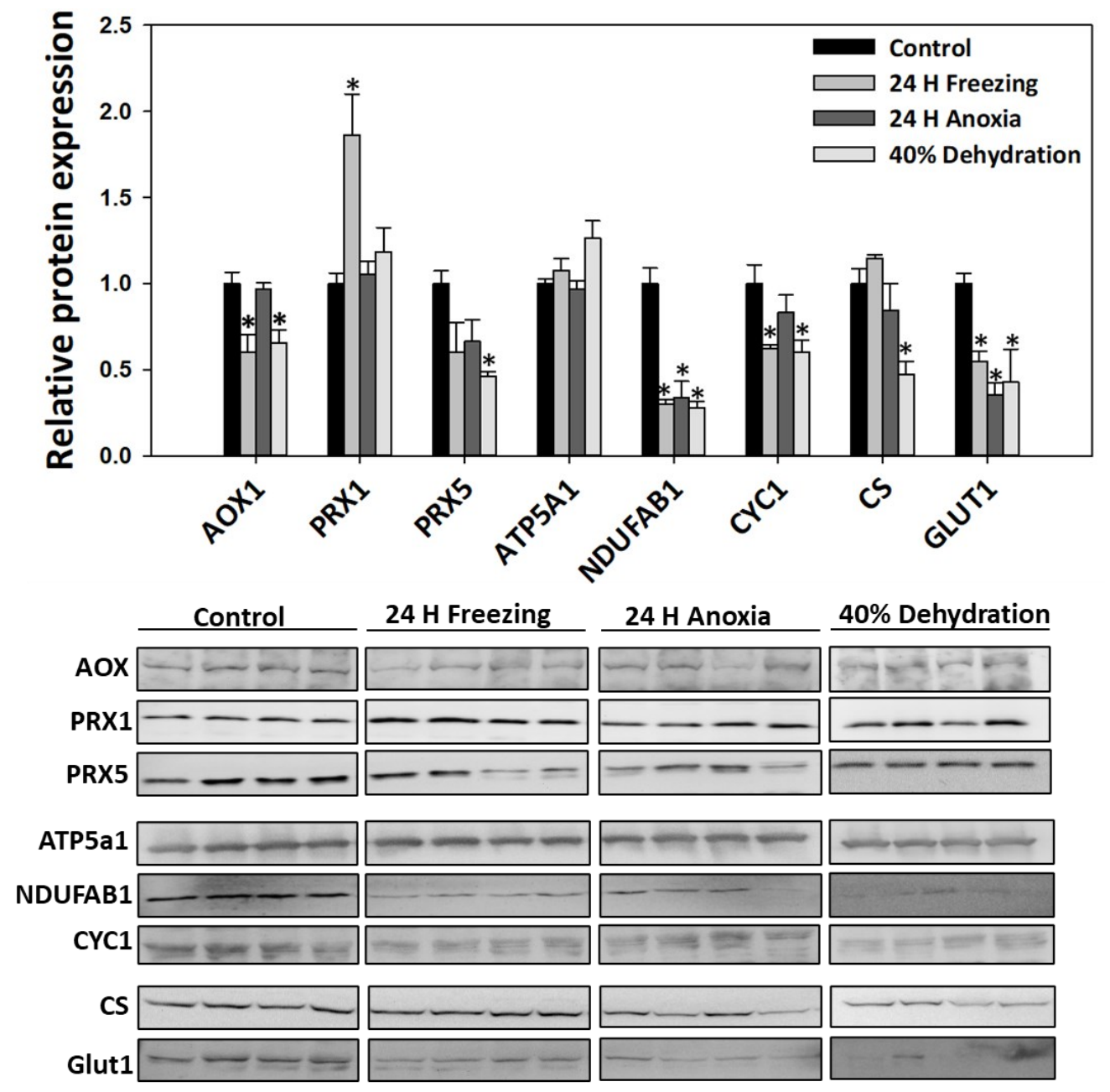

Figure 4.7: Relative protein expression levels of select ETS1 downstream targets measured by western blotting in wood frog heart. For more information, see Figure 4.2. 


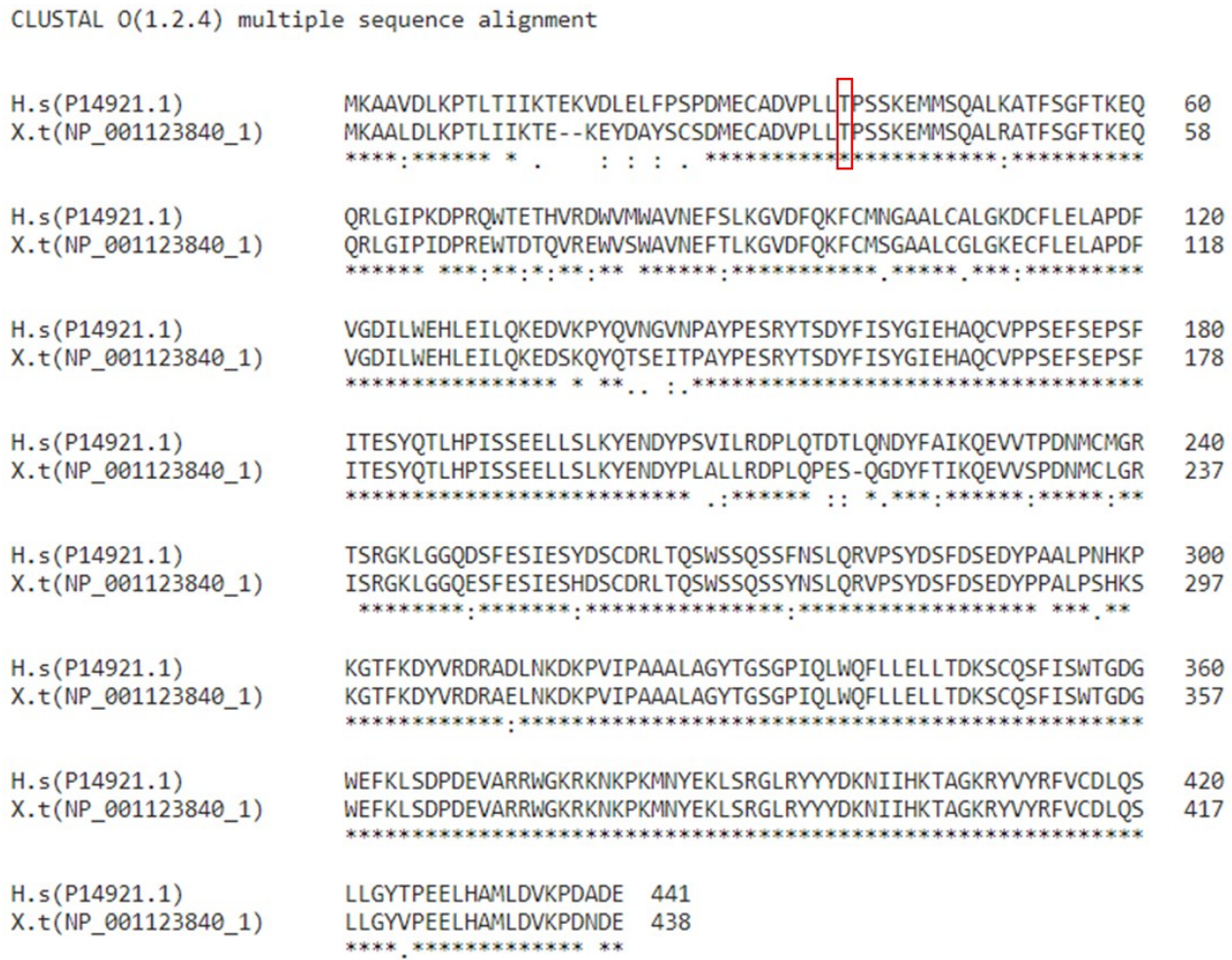

Figure S4.1: Alignment showing similarities between the ETS1 protein sequence of Xenopus tropicalis (NP_001123840_1) and Humans (P14921.1), generated using Clustal Omega. Conserved phosphorylation site T38 is shown in red. 
Table S4.1: Shows different posttranslational modifications that could modulate ETS-1 activity. Confirmed phosphorylation site information was retrieved from PhosphoSitePlus ${ }^{\circledR}$ (https://www.phosphosite.org/). Potential SUMOylation and acetylation sites on Xenopus laevis ETS-1 were predicted using the Bio CUCKOO program (http://gps.biocuckoo.cn/).

\begin{tabular}{lll} 
Protein & Modification & Site \\
\hline ETS1 & Phosphorylation & T38, T251, S257, S276, S282, Y283, S285 \\
& Acetylation & K2, K89, K433 \\
& SUMOylating & K14, K198, K224, K433 \\
&
\end{tabular}




\section{Chapter 5: General discussion}




\subsection{General discussion}

Harsh environmental conditions could be challenging to some animals living in the wild. Extreme temperatures, limited food availability, or oxygen deprivation could result in death. Animals that survive these unfavorable conditions do so by employing sophisticated adaptive mechanisms that include responses at the physiological, biochemical, and molecular levels. Of interest to this thesis was understanding the molecular mechanisms behind vertebrate freezing tolerance by using the freeze-tolerant wood frog, Rana sylvatica, as a model organism. Understanding the plasticity involved in supporting survival under these conditions will increase our knowledge and could potentially help in finding solutions to various medical complications such as diabetes, ischemic stroke, or prolonging organ storage time before transplantations by designing interventions that mimic those found in natural adaptive responses.

Freezing is a multifactorial stress, and requires adaptations to deal with hyperglycemia, dehydration, and anoxia [1,2]. Each of these conditions are associated with series of complications that if not dealt with appropriately could result in cell death. For example, ice crystal growth could damage the lumen of the capillaries, leading to the loss of vascular integrity, resulting in internal bleeding upon thawing [3,4]. Extreme dehydration could also rupture cell membranes, and extreme increases in cytoplasmic ionic strength could result in disruption in various cellular processes [5]. Prolonged anoxia (or ischemia in case of freezing) deprives cells from oxygen, increases the levels of reactive oxygen species (ROS), and prevents waste cleanup from organs. Moreover, while accumulating large quantities of glucose as cryoprotectant is beneficial in cells, glucose is prone to autooxidation and under conditions of oxidative stress, could result in 
the formation of advanced glycation end products (AGEs) [6,7]. In addition to enduring these stresses, recovery from each condition is also associated with potential damage, chiefly an outburst of ROS production upon reperfusion, that must be mitigated to ensure survival. The commonality amongst freezing, anoxia and dehydration stresses is the low or absent availability of oxygen, which forces cells to switch from aerobic metabolism to generating energy in an anaerobic manner. Although copious amounts of glucose are available (especially under freezing or dehydration conditions, less so in response to anoxia), the amount of energy generated anaerobically is far less than the amount generated under aerobic conditions and cannot provide enough energy to sustain normal metabolic rates. As such, wood frogs are hypothesized to reduce their metabolic rates to a fraction of normal in a process called metabolic rate depression (MRD) to balance energy production with energy utilization in their cells. While data on metabolic rates are scarce for wood frogs, and extremely difficult to measure for frozen animals, other ectotherms are known to show significant reductions in their overall metabolism in response to anoxia. For example, adult turtles (Chrysemys picta belli) submerged in anoxic water at $3^{\circ} \mathrm{C}$ reduce their metabolism by $\sim 90 \%$ and survive for months by relying only on their internal fuels [8]. Similarly, frogs that overwinter in ice-locked ponds, or toads or adult frogs that live in arid regions in the world reduce their metabolism down to $\sim 10 \%$ of their normal rates, to deal with unfavorable environmental conditions $[9,10]$. Moreover, some species of lizards (Lacerta vivipara) have also been reported to decrease their metabolic rates drastically in response to freezing [11], although the accuracy of measuring metabolic rates from a frozen organisms is debatable. 
Regulation of MRD occurs at multiple biological levels in stress, tissue, and time dependent manner. In chapter 2, regulation of autophagy was studied to document responses to 24-hour anoxia, 4-hour reoxygenation, $40 \%$ dehydration, and full rehydration in wood frog liver and skeletal muscle. Autophagy is an energy expensive, catabolic process where defective or dysfunctional cytoplasmic organelles and macromolecules are engulfed and degraded to generate metabolic substrates in response to different stimuli [12]. There are conflicting studies on the benefits of autophagy in response to different conditions. For example, it was shown that excessive autophagy in hippocampal neurons in response to hypoxia/ischemia resulted in autophagic cell death, and that inhibiting autophagy reversed this process [13]. Another study reported that inhibition of autophagy exacerbated ischemia-induced liver damage, and induction of this process reduced ROS levels and prevented necrotic cell death [14]. Not surprisingly, activation of autophagy depends on the amount of energy available in the cell since this process is highly energy expensive and requires the activation of numerous complexes; a process that may not be possible in conditions under many environmental stress conditions.

Autophagy is a multistage process that is divided into four phases: initiation, nucleation, elongation, and fusion [15]. Each of these stages requires the formation of complexes, and studies have used some of the proteins involved in these complexes as markers of autophagic flux. The induction of these proteins depends on the activity of specific transcription factors including but not limited to Transcription factor EB (TFEB) $[16,17]$. Studies of this transcription factor revealed that TFEB binds to various genes involved in autophagosome formation and lysosomal biogenesis and, therefore, for 
autophagy to be active, this transcription factor must be active [16,17]. At the posttranscriptional level, autophagy is also regulated by various microRNAs that strictly regulate the translation of various mRNA transcripts encoding essential proteins involved in the complexes used for autophagosome formation and lysosome formation [18]. At the protein level, autophagy or lysosomal biogenesis are regulated by various posttranslational modifications (PTMs) [19]. Indeed, autophagosome formation is regulated by the Unc-51 like autophagy activating kinase 1 (ULK1), which is either activated by AMPK (AMP activated protein kinase)-mediated phosphorylation at S555 or inhibited by mTOR (mechanistic target of rapamycin)-mediated phosphorylation at S758 [20,21]. Other components of autophagy (autophagy regulating proteins, ATGs) are also subject to PTMs, namely phosphorylation, acetylation, ubiquitination and lipidation, where the specificity of each PTM can dictate protein function [22,23]. In Chapter 2, regulation of autophagy was investigated at the transcriptional level by studying the activity of TFEB, at the post-transcriptional level by studying various autophagy regulating microRNAs (autophagomiRs), and at the post-translational level by reversible protein phosphorylation.

In general, exposure to anoxia or reoxygenation reduced the protein levels of the majority of autophagy markers, with the exception of ULK1 where total levels as well as levels of its active (p-ULK1 S555) and inactive (P-ULK1 S758) forms increased under both conditions. Responses to dehydration showed different trends with levels of select autophagy markers (p-beclin 1 S93), p62 and the LC3BII/I ratio increased significantly compared to the control group. These changes were also accompanied by an increase in total, active and inactive forms of ULK1 in the same tissue in response to dehydration. 
Changes observed under anoxia/reoxygenation and dehydration/rehydration coincided with the DNA binding activity of TFEB, a transcription factor that carries out the transcription of numerous targets including, but not limited to, the transcription of p62. However, despite an increase in the DNA binding levels of TFEB in response to anoxia and dehydration, levels of ATPV0D1 (a lysosomal marker under TFEB control) remained the same. Interestingly, control of autophagy at the post-transcriptional level showed a tissue and stress-dependent regulation. For example, while only 2 microRNAs were increased in response to anoxia in wood frog liver, the majority of the microRNAs measured showed a decreasing trend in response to dehydration, a finding that correlates with the increasing trends of their downstream targets under the same conditions. Altogether these changes suggest that autophagosome formation in liver is greatly attenuated in response to anoxia/reoxygenation (probably at the transcriptional level) but responds to dehydration at the post-translational level. Responses to dehydration by liver do suggest the formation of small numbers of autophagosomes, probably in an effort to engulf and process damaged cytoplasmic cargo under stress, but the evidence suggests that fusion with lysosomes is postponed until more favorable conditions arise.

Interestingly, the comparable analysis in skeletal muscle showed significant reductions in most autophagosome and lysosome targets in response to anoxia/reoxygenation as well as dehydration/rehydration, where total levels of ULK1 were significantly reduced in response to all conditions. Regulated reduction in protein levels during anoxia/reoxygenation appears to occur at the transcriptional level, as supported by the decrease in TFEB transcriptional activity in response to these conditions. Indeed, it was reported that activation of EZH2 (enhancer of zeste homolog 
2), a methyl transferase enzyme that inhibits gene expression by methylating promoter regions, targets several autophagy genes and causes their silencing [24]. Likewise, inactivation of EZH2 relieves that inhibition and induces the expression of several proautophagy proteins including, but not limited to, ATG5 and ATG7 [24]. Moreover, the arginine methyltransferase 1 (CRM1) was shown to induce autophagy by enhancing the transcriptional activity of TFEB, thereby promoting the expression of autophagy and lysosomal genes [25]. Studies of epigenetic regulation of anoxia and dehydration tolerance in wood frogs are beginning to be evaluated; however, studies on other hypometabolic models have demonstrated the involvement of epigenetics in regulating multiple aspects of MRD, where markers that promote global gene suppression are induced in response to stress [26-29]. Regulation of autophagy at the transcriptional level in muscles from anoxic frogs is also supported by a general decrease in levels of autophagomiRs. In theory, if the downstream targets are not transcribed, then no regulation is required to inhibit their mRNA translation at the post-transcriptional level.

Results for skeletal muscle from dehydrated/rehydrated frogs showed a different response. Whereas the DNA binding activity of TFEB did not change in response to dehydration, it decreased significantly in response to rehydration, and combined with decreases in protein levels of select downstream targets, this suggests that perhaps regulation of autophagy occurs at the post-transcriptional level in this tissue. Indeed, there was an overall increase in the expression of autophagomiRs in muscle from dehydrated/rehydrated wood frogs, thereby further supporting the hypothesis that microRNAs are in part responsible for reducing autophagic activities in muscle from frogs exposed to dehydration/rehydration treatments. 
Maintaining very low levels of autophagic flux or inhibiting autophagy all together appears to be an appropriate response that supports MRD for many reasons. First, autophagy is induced in response to multiple stimuli including but not limited to nutrient deprivation or oxidative stress. As discussed, wood frogs experience no nutrient limitations as they have access to high levels of glucose (more so during dehydration/rehydration) during stress. Although the primary function of glucose is cryoprotection, it could also be used for energy production when required. Moreover, despite facing conditions that promote oxidative stress, wood frogs and other animals capable of undergoing MRD, have higher levels of antioxidant enzymes, heat shock proteins, and antioxidant metabolites [30-34]. In fact, a study demonstrated that freezetolerant species such as Rana sylvatica, have higher basal levels of antioxidant enzymes compared to a less stress-tolerant aquatic frog [34]. Moreover, there are no studies to date that report any measurable damage in wood frogs following stress or recovery. As such, even if faced with a surge in oxidants, the wood frog's ability to launch an antioxidant response renders it unassailable to oxidative damage.

Second, the induction of autophagosome formation and lysosomal biogenesis requires a plethora of complexes that, although recyclable, are energetically expensive to synthesize and maintain. Given that protein translation is greatly suppressed during MRD [35], maintaining the translation of complexes involved in autophagy becomes extremely energy expensive. Moreover, even if these proteins are translated adequately and in a timely manner, most autophagy proteins depend on post-translational modifications for activation. Indeed, acetylation (the conjugation of an acetyl group, donated from acetylCoA, to a protein) plays a significant role in activating various autophagy proteins [36]. 
Pyruvate dehydrogenase, the enzyme complex that generates acetyl-CoA, is significantly inhibited during freezing and anoxia in wood frogs [37]. This reduction depletes the cell of its pool of free acetyl-CoA molecules to use for activating various autophagy proteins and, thus, provides another line of evidence that autophagy is significantly reduced under stress conditions. Third, some animals capable of undergoing MRD have been reported to have higher basal autophagic flux, that could partly be responsible for stress survival [38]. While more studies are warranted, it is possible that wood frogs also have higher basal levels of autophagy compared to non-stress tolerant frogs. A significant reduction in autophagic flux appears to be a pro-survival mechanism used by wood frogs in response to anoxia/reoxygenation, dehydration and rehydration treatments. Altogether, results in Chapter 2 support the first hypothesis proposed in this thesis; that, autophagosome formation and lysosomal biogenesis is greatly attenuated in response to stress in wood frogs, and the degree of this attenuation occurs in a tissue and stress dependent manner.

In Chapter 3, the involvement of RAGE in regulating transcriptional responses to angiogenesis (the formation of new blood vessels sprouting from pre-existing ones) was studied in liver of wood frogs exposed to 24-hour freezing, 8-hour thawing, 24-hour anoxia, 4-hour aerobic recovery, $40 \%$ dehydration and full rehydration. Freezing, thawing, dehydration and or rehydration treatments could potentially rupture capillaries, resulting in loss of vascular integrity in wood frog organs. Moreover, although protective mechanisms are put in place, the presence of ice crystals in the extracellular spaces (such as the lumen of capillaries) could result in tissue damage during freezing and excessive bleeding upon recovery. In addition, forming new blood vesicles early in response to 
stress could also potentially help with the distribution of glucose from the liver to all other organs in the body. In addition, numerous studies have reported the benefits of activating angiogenesis in response to stress. For example, induction of angiogenesis in hearts exposed to ischemic stress or in response to tissue injury reduced the progression of cardiac infarction and accelerated wound healing $[39,40]$. As such, it was of interest to determine whether angiogenesis occurs in wood frogs, and if so, what molecular mechanisms regulate this process.

As mentioned earlier, freezing, thawing, dehydration, and rehydration (and to a lesser degree anoxia and reoxygenation) are hyperglycemic events, and wood frogs generate large quantities of glucose to use as a cryo- or osmo-protectant under these conditions $[5,41]$. However, despite offering protective benefits, excessive glucose levels in presence of oxidative stress results in the production and accumulation of AGEs [6,7]. Indeed, a previous study in our laboratory showed that whereas levels of glycated serum albumin did not change in response to freezing, levels had increased significantly 7 days post-thawing [42]. Another protein that is involved in regulating angiogenesis is the high mobility group box 1 (HMGB1) [43]. Under normal conditions, this non-histone, DNAbinding protein resides in the nucleus, but upon stimulation, it becomes acetylated and is exported into the cytoplasm [44]. In the cytoplasm, HMGB1 can regulate various processes, including autophagy, by binding to and activating Beclin 1 [45]. Indeed, the unchanged levels of acetylated HMGB1 seen in Chapter $\mathbf{3}$ in response to anoxia, reoxygenation, dehydration, and rehydration correlates with low levels of Beclin 1 activation, and ultimately a reduction in autophagosome formation in liver of wood frogs as seen in Chapter 2. Once in the cytoplasm, acetylated HMGB1 will associate with 
secretory vesicles and get transported into the extracellular space where it can associate with different receptors including the receptor for AGE (RAGE) [46]. Association of AGE and HMGB1 results in the activation of RAGE and subsequent signal transduction events that lead to the expression and activation of the early growth response 1 (EGR1) and the E26 transformation-specific sequence 1 (ETS1) transcription factors [47,48]. These transcription factors regulate numerous genes, including various pro- and antiangiogenic targets.

Results from Chapter 3 demonstrate that AGE generation is greatly reduced in response to freezing, thawing, anoxia, and dehydration, probably owning to the strong activation of potent antioxidant responses under these conditions in wood frogs. Indeed, numerous studies have demonstrated the up regulation of various antioxidant enzymes including, but not limited to, aldo-keto reductases and glutathione S-transferases, as well as antioxidant metabolites in response to stress in wood frogs $[33,34,49]$. These enzymes have been shown to promote AGE clearance and prevent the accumulation of AGEadducts in the cell [50]. Although low, the presence of these adducts could still result in the activation of RAGE. In Chapter 3, protein levels of RAGE were shown to be strongly induced in response to freezing, anoxia, reoxygenation, dehydration and rehydration in wood frog liver. Together, results in Chapter $\mathbf{3}$ demonstrate the potential activation of RAGE signaling under stress conditions, which prompted further investigation of angiogenesis.

The results presented in this thesis show that the nuclear levels and DNA binding activity of ETS1 and EGR1 are regulated in a stress-dependent manner. For example, 
while the nuclear levels of ETS1 were upregulated in response to freezing, anoxia, dehydration, and rehydration, EGR1 levels were upregulated only in response to freezing and thawing. Moreover, two transcriptional repressors of EGR1, NAB1 (NGFI-A binding-1, constitutive) and NAB2 (inducible), also appeared to be localized to the nucleus in a stress-dependent manner. Whereas promoting the nuclear localization of a transcription factor along with its inhibitor may seem counterintuitive, it may be a strategic response by wood frogs. This is because wood frogs, along with many animals that undergo MRD, induce the expression of essential and pro-survival factors necessary for long term stress survival or in response to stages of recovery, before the full onset of stress when energy levels are high. However, wood frogs also have mechanisms that allow them to maintain the activity of these factors at minimum until they are required in the cell. Indeed, the transcriptional responses controlled by ETS1 and EGR1 also demonstrate this phenomenon, more so in response to freezing and dehydration. For example, angiopoietin 1 (ANGPT1) and ANGPT2 are two important factors that regulate angiogenesis by associating with receptors Tie1 and Tie2 [51,52]. However, whereas ANGPT1 promotes vascular remodeling, vascular enlargement, and endothelial cell (EC) migration, and stabilization of newly formed vasculature, ANGPT2 promotes vascular destabilization, enhances sprouting and disrupts the recruitment of pericytes, thereby preventing the stabilization of the vasculature [51,53]. Whereas both ANGPTs are needed for angiogenesis, their presence should be regulated in an inverse manner to first promote vascular destabilization and sprouting during the initiation of angiogenesis (ANGPT2), and then promote vascular remodeling, EC migration and vascular stabilization at the end of angiogenesis (ANGPT1). Wood frogs upregulate both factors in anticipation of 
potential damage in response to freezing and dehydration but less so in response to anoxia treatment. Indeed, dehydration and freezing and their respective recoveries could result in architectural damage to capillaries or cause tissue injury. Therefore, having both these factors available can enable frogs to use them as necessary whenever needed. Other angiogenic factors such as VEGFR1 (vascular endothelial growth factor receptor 1) and VE-cad (vascular endothelial cadherin) are also regulated in the same manner. The angiogenic responses by ETS1 and EGR1 appear to be regulated in a temporal manner, where the induction of downstream angiogenic factors appear to occur before the full onset of stress in anticipation to potential damage that may occur during/after stress or in early stages of recovery. Altogether, results shown in Chapter $\mathbf{3}$ demonstrate the potential involvement of the HMGB1/RAGE axis in inducing and activating transcriptional responses by ETS1 and EGR1 in a time- and stress-dependent manner, resulting in the induction of both pro- and anti-angiogenic factors in wood frogs. These findings support the original hypothesis, where temporal induction of angiogenesis as a preparative response may be necessary to repair damaged capillaries or promote wound healing in wood frogs, particularly after a prolonged freezing bout.

In Chapter 4, the involvement of RAGE signaling in inducing ETS1 activation was examined in wood frog heart after exposure to 24-hour freezing, 24-hour anoxia or $40 \%$ dehydration. In addition to regulating angiogenesis, ETS1 also regulates the expression of other genes encoding various antioxidant enzymes, components of the mitochondrial electron transport chain, glucose uptake and energy metabolism [54-57]. As a transcriptional activator, ETS1 was shown to promote the expression of aldehyde oxidase 1 (AOX1), peroxiredoxin 1, (PRX1), PRX5, glucose transporter 1 (GLUT1) [54- 
56]. Interestingly, in addition to its role as a transcriptional activator, ETS1 can also act as a transcriptional repressor of several downstream genes [58,59]. For example, studies have shown that induction of ETS1 resulted in the downregulation of select nuclearencoded mitochondrial genes such as the NADH dehydrogenase (ubiquinone 1) $\alpha / \beta$ subcomplex 1 (NDUFAB1), cytochrome c1 (CYC1), and ATP synthase $\mathrm{H}^{+}$transporting mitochondrial F1 complex, $\alpha$ subunit 1 cardiac muscle (ATP5A1) $[55,56]$. Mitochondria are the powerhouse of the cell and are responsible for generating the majority of ATP available under aerobic conditions. However, when oxygen is scarce, mitochondria become a liability to cells since they become the major producers of ROS. Under these conditions, the $\mathrm{F}_{1} \mathrm{~F}_{0}-\mathrm{ATPase}$ commits "cellular treason" and uses a portion of the available ATP to maintain the proton-motive force in the cell [60]. As such, animals that survive under oxygen-deprived conditions have adapted a mechanism that allows them to limit ATP hydrolysis by inhibiting the activity of the $\mathrm{F}_{1} \mathrm{~F}_{0}$-ATPase, or by reducing proton conductance of the inner mitochondrial membrane [60]. Indeed, the activity of $\mathrm{F}_{1} \mathrm{~F}_{0}$-ATPase was shown to be significantly reduced in brains of anoxic turtles [61], and in mitochondria isolated from frogs exposed to anoxia [60]. Therefore, it is possible that this inhibition can be at least partially traced to the transcriptional level via downregulation of the transcription of nuclear encoded mitochondrial proteins.

In Chapter 3, the involvement of HMGB/AGE/RAGE axis in activating ETS1 was investigated in wood frog liver and in Chapter 4, the involvement of the same pathway in inducing the activity of ETS1 was examined in the heart of wood frogs. A preliminary study by our group, using cDNA arrays, demonstrated that exposure to freezing induced the expression of RAGE in wood frog hearts [1]. Hearts from freeze or 
dehydration exposed frogs accumulate large quantities of glucose, as such, they are also prone to accumulating large quantities of AGEs and AGE-adducts. Results in Chapter 4 demonstrate that AGE levels are reduced in response to freezing but are unaffected by exposure to anoxia or dehydration. Moreover, both total protein and acetylated HMGB1 levels were elevated in response to all treatment conditions, which coincides with the same increasing trends seen in RAGE protein levels. These results indicate that HMGB1, and to a lesser degree AGE, could induce a RAGE-mediated response. However, investigation into downstream targets of RAGE that function upstream of ETS1 activation demonstrated two things: (1) ETS1 phosphorylation in response to dehydration may be mediated by the canonical way of RAGE/ETS1 pathway activation, and (2) ETS1 activation may be regulated in a temporal manner by alternative factors in response to freezing and anoxia. Regardless of the activation mode, results in Chapter 4 demonstrated that both total ETS1 and the active form (p-ETS1 T38) show strong DNA binding activity in response to freezing and dehydration but not in response to anoxia treatment. This pattern of activation correlated well with the expression patterns of AOX1 under the same conditions. However, in this case, ETS1 appears to act as a transcriptional repressor to AOX1. This is not surprising, given the role of AOX1 in inducing the production of superoxide anions, reactive nitrogen species, and ROS in the cell [62]. Therefore, to eliminate the production of these prooxidant species, it is logical for wood frogs to use ETS1 as a transcriptional repressor to prevent the expression of AOX1 in the heart in an effort to reduce oxidative damage. It appears that ETS1 induced the expression of PRX1 in response to freezing but not anoxia or dehydration. In addition, PRX5 expression patterns were not affected by ETS1 activation, and in fact 
were reduced in response to dehydration. Under metabolically depressed states, it is possible that cells would prioritize the production of more potent antioxidant enzymes in larger quantities, rather than activating less potent ones such as PRX5. In response to all three conditions, ETS1 appears to reduce the expression of NDUFAB1 and CYC1; which correlate with results presented in other published studies. These two proteins are components of the electron transport chain, and given that the activity of the electron transport chain is greatly reduced in response to low oxygen levels, it is logical to assume that decreasing the transcription of these subunits by ETS1 is a protective response initiated by wood frogs. However, increased DNA binding of ETS1 in response to freezing and dehydration had no effect on the expression profile of ATP5A1, a component of the F1F0-ATPase complex. Given that previous studies on anoxic frogs report significant reduction in the activity of this complex, it is possible that wood frogs attenuate its activity downstream of its transcription, potentially at the post-translational level by PTMs. Similarly, overexpression of ETS1 should inhibit the expression citrate synthase (CS) $[55,56]$. In Chapter 4, it was demonstrated that activation of ETS1 resulted in a reduction of CS expression only in response to dehydration and had no effect on its expression in response to freezing despite an increase in ETS1 DNA binding activity observed under the same condition. This demonstrated that CS could be potentially regulated at the post-translational level in wood frogs, downstream of ETS1 activation. Lastly, the increase in ETS1 DNA binding should have increased the expression of GLUT1, but the opposite was observed; when DNA binding levels of ETS1 increased, levels of GLUT1 decreased. As mentioned earlier, under a hypmetabolic state when energy levels are limited, inducing the expression of more potent proteins that do a 
similar job is favored over inducing the expression of similar targets with lower activity levels. As such, given that transcript levels of glut 4 were induced in the heart, then it may be unnecessary for wood frogs to alter the expression of GLUT1 if its function is similar to another highly expressed transporter [63]. Therefore, while ETS1 could have in theory activated GLUT1, it is possible that in the case of wood frogs, it acted as a transcriptional repressor to prevent the expression of GLUT1 in the heart to conserve energy. While these assumptions are logical, further experiments are needed to confirm these suggestions. Altogether, results in Chapter 4 partly support the original hypothesis where ETS1 was proposed to act as a transcriptional activator of some antioxidant enzymes, but act as a transcriptional repressor of select nuclear-encoded mitochondrial proteins, antioxidant enzymes, and metabolic targets.

\subsection{Future directions}

While this thesis provides a comprehensive overview on the regulation of several signaling pathways in wood frogs in response to freezing, anoxia or dehydration, along with the respective recoveries from these stresses, further experiments could provide a more definitive answer to the proposed hypotheses. First, the results of Chapter $\mathbf{2}$ clearly demonstrate a significant reduction in autophagosome formation and lysosomal biogenesis in response to anoxia/reoxygenation and to a lesser degree in response to dehydration and rehydration in liver, and in response to both anoxia/reoxygenation and dehydration/rehydration in skeletal muscle. However, visualizing the numbers of autophagic vacuoles formed could provide a more definitive answer to autophagic flux under these conditions. For example, whereas our results indicate a significant reduction, the levels of the proteins involved in both autophagosome formation and lysosomal 
biogenesis are not fully depleted. As such, it is still possible for cells to maintain a low level of autophagic flux to clear engulfed cargo. Therefore, visualizing different stages of autophagosome formation using transmission electron microscopy (TEM) would provide more information about autophagic flux in wood frogs. This method has been widely used in autophagy research to detect different autophagic compartments in cells $[38,64]$. Although identifying autophagic vacuoles is extremely difficult and requires a welltrained individual, using this method as compared to other microscopy methods is preferred because it does not require the utilization of highly specific antibodies, which may not be commercially available or specific to frogs. In combination with the current results shown in Chapter 2, having TEM data would add another layer of confidence in assuming that autophagic flux is either inhibited or occurs at very low levels in wood frogs exposed to stress.

Given that the wood frogs are currently not genome sequenced, it is extremely difficult to detect the transcript levels of select downstream markers. Although proteins are the functional units of the cell, having the transcript levels would enable us to determine the biological level at which these targets are being regulated. This may soon be a possibility since the wood frog genome is currently being sequenced. Having a comprehensive list of all targets regulated by certain transcription factors would be useful, because it will allow researchers to investigate how a given pathway is regulated at the transcriptional level and whether a defect in transcription is responsible for downstream events. As such, chromatin immunoprecipitation and subsequent massive parallel sequencing (ChIP-seq) can be used to generate a genome-wide map of gene promotor regions that are occupied by specific transcription factors studied in this thesis 
(TFEB, ETS1 and EGR1) [65]. In combination with transcription factor localization studies, DNA binding activity and measurement of protein levels of select targets, ChIPseq data can provide insightful information on whether the targets of interest are regulated at the transcriptional level by the transcription factor in question. Combining the ChIP-seq data from ETS1 and measuring the transcript levels of select downstream genes would provide more information on whether ETS1 acts as a transcriptional activator or a transcriptional repressor to these targets. Moreover, the obtained ChIP-seq information could provide insight into novel gene promotors that are being occupied by a specific transcription factor; thereby creating new avenues for future research.

Lastly, as demonstrated by target prediction programs in Chapter 4, ETS1 (and other transcription factors) can be post-translationally modified, and the identity of these modifications can affect the intracellular localization and the transcriptional activity of this transcription factor. Immunoprecipitation (IP) is a relatively easy and cost-effective way of purifying a specific protein from a sample using commercially available antibodies [66]. Combining the IP products with western blotting using pan antibodies that detect various modifications reported by the prediction software (e. g. methylation, nitrosylation, acetylation, ubiquitination, SUMOylation, etc) could provide a general idea of the identity and the relative levels of these modifications on ETS1 in response to stress. Moreover, using the same IP products to measure the DNA binding levels of a given transcription factor by utilizing an antibody that detects a specific PTM, could in theory give information on whether this modification has a role in regulating the DNA binding activity of the transcription factor in question. Altogether, performing these simple experiments could lead to finding novel PTMs for a given transcription factor, and 
increase our understanding of the various modifications used by wood frogs to regulate enzymatic activities in response to stress.

\subsection{Concluding remarks}

In conclusion, the findings in this thesis provide novel information on the regulatory mechanisms involved in controlling autophagy, angiogenesis, energy metabolism and select antioxidant defense responses in wood frogs exposed to environmental stress. With regards to autophagy, the results show that autophagosome formation and lysosomal biogenesis is greatly attenuated in response to anoxia/reoxygenation and to a lesser degree in response to dehydration and rehydration in liver, whereas greater inhibition of these two processes in response to anoxia/reoxygenation and dehydration/rehydration was seen in skeletal muscle. Moreover, current results also show that angiogenesis is regulated in a temporal and stress-dependent manner, where wood frogs increase the expression of certain pro- and anti-angiogenic factors in anticipation of potential damage to capillaries or injuries in tissues. Lastly, results in this thesis also show that reduction in the activity of the electron transport chain could be regulated, in part, at the transcriptional level, where increased ETS1 DNA binding would inhibit the expression of nuclear encoded mitochondrial proteins. Similarly, activation of ETS1 also results in inhibition of enzymes involved in ROS production, while promoting the expression of antioxidant enzymes that alleviate this process. Overall, findings in this study demonstrate the highly regulated and extremely sophisticated mechanisms involved in controlling metabolic rate depression and stress survival in wood frogs. 


\subsection{References- Chapter 5}

[1] K.B. Storey, Strategies for exploration of freeze responsive gene expression: Advances in vertebrate freeze tolerance, Cryobiology. 48 (2004) 134-145. https://doi.org/10.1016/j.cryobiol.2003.10.008.

[2] K.B. Storey, J.M. Storey, Freeze tolerant frogs: Cryoprotectants and tissue metabolism during freeze-thaw cycles, Can. J. Zool. 64 (1986) 49-56. https://doi.org/10.1139/z86-008.

[3] K.B. Storey, J.M. Storey, Physiology, biochemistry and molecular biology of vertebrate freeze tolerance: The wood frog, in: B.J. Fuller, N. Lane, E.E. Benson (Eds.), Life a Frozen State, CRC Press, Boca Raton, 2004: pp. 243-274.

[4] K.B. Storey, J.M. Storey, Molecular physiology of freeze tolerance in vertebrates, Physiol. Rev. 97 (2017) 623-665. https://doi.org/10.1152/physrev.00016.2016.

[5] T.A. Churchill, K.B. Storey, Dehydration tolerance in wood frogs: A new perspective on development of amphibian freeze tolerance, Am. J. Physiol. Integr. Comp. Physiol. 265 (1993) R1324-R1332.

https://doi.org/10.1152/ajpregu.1993.265.6.R1324.

[6] A.W. Stitt, J.E. Moore, J.A. Sharkey, G. Murphy, D.A.C. Simpson, R. Bucala, H. Vlassara, D.B. Archer, Advanced glycation end products in vitreous: Structural and functional implications for diabetic vitreopathy., Invest. Ophthalmol. Vis. Sci. 39 (1998) 2517-23. http://www.ncbi.nlm.nih.gov/pubmed/9856760.

[7] M. Fournet, F. Bonté, A. Desmoulière, Glycation damage: A possible hub for major pathophysiological disorders and aging, Aging Dis. 9 (2018) 880. 
https://doi.org/10.14336/AD.2017.1121.

[8] C. V. Herbert, D.C. Jackson, Temperature effects on the responses to prolonged submergence in the turtle Chrysemys picta bellii. II. Metabolic rate, blood acidbase and ionic changes, and cardiovascular function in aerated and anoxic water, Physiol. Zool. 58 (1985) 670-681.

https://doi.org/10.1086/physzool.58.6.30156071.

[9] P.H. Donohoe, T.G. West, R.G. Boutilier, Respiratory, metabolic, and acid-base correlates of aerobic metabolic rate reduction in overwintering frogs, Am. J.

Physiol. Integr. Comp. Physiol. 274 (1998) R704-R710.

https://doi.org/10.1152/ajpregu.1998.274.3.R704.

[10] K.B. Storey, Life in the slow lane: Molecular mechanisms of estivation., Comp. Biochem. Physiol. A. Mol. Integr. Physiol. 133 (2002) 733-54. https://doi.org/10.1016/S1095-6433(02)00206-4.

[11] Y. Voituron, B. Verdier, C. Grenot, The respiratory metabolism of a lizard (Lacerta vivipara) in supercooled and frozen states, Am. J. Physiol. Integr. Comp. Physiol. 283 (2002) R181-R186. https://doi.org/10.1152/ajpregu.00378.2001.

[12] Z. Zhang, M. Guo, S. Zhao, W. Xu, J. Shao, F. Zhang, L. Wu, Y. Lu, S. Zheng, The update on transcriptional regulation of autophagy in normal and pathologic cells: A novel therapeutic target, Biomed. Pharmacother. 74 (2015) 17-29. https://doi.org/10.1016/j.biopha.2015.06.003.

[13] M. Koike, M. Shibata, M. Tadakoshi, K. Gotoh, M. Komatsu, S. Waguri, N. Kawahara, K. Kuida, S. Nagata, E. Kominami, K. Tanaka, Y. Uchiyama, 
Inhibition of autophagy prevents hippocampal pyramidal neuron death after hypoxic-ischemic injury, Am. J. Pathol. 172 (2008) 454-469. https://doi.org/10.2353/ajpath.2008.070876.

[14] K. Sun, X. Xie, Y. Liu, Z. Han, X. Zhao, N. Cai, S. Zhang, J. Song, L. Wei, Autophagy lessens ischemic liver injury by reducing oxidative damage, Cell Biosci. 3 (2013) 26. https://doi.org/10.1186/2045-3701-3-26.

[15] C. He, D.J. Klionsky, Regulation mechanisms and signaling pathways of autophagy, Annu. Rev. Genet. 43 (2009) 67-93. https://doi.org/10.1146/annurevgenet-102808-114910.

[16] M. Palmieri, S. Impey, H. Kang, A. di Ronza, C. Pelz, M. Sardiello, A. Ballabio, Characterization of the CLEAR network reveals an integrated control of cellular clearance pathways, Hum. Mol. Genet. 20 (2011) 3852-3866. https://doi.org/10.1093/hmg/ddr306.

[17] M. Sardiello, M. Palmieri, A. di Ronza, D.L. Medina, M. Valenza, V.A. Gennarino, C. Di Malta, F. Donaudy, V. Embrione, R.S. Polishchuk, S. Banfi, G. Parenti, E. Cattaneo, A. Ballabio, A Gene Network Regulating Lysosomal Biogenesis and Function, Science (80-. ). (2009). https://doi.org/10.1126/science.1174447.

[18] L.B. Frankel, A.H. Lund, MicroRNA regulation of autophagy, Carcinogenesis. 33 (2012) 2018-2025. https://doi.org/10.1093/carcin/bgs266.

[19] Y. Xie, R. Kang, X. Sun, M. Zhong, J. Huang, D.J. Klionsky, D. Tang, Posttranslational modification of autophagy-related proteins in macroautophagy, 
Autophagy. 11 (2015) 28-45. https://doi.org/10.4161/15548627.2014.984267.

[20] W. Tian, W. Li, Y. Chen, Z. Yan, X. Huang, H. Zhuang, W. Zhong, Y. Chen, W. Wu, C. Lin, H. Chen, X. Hou, L. Zhang, S. Sui, B. Zhao, Z. Hu, L. Li, D. Feng, Phosphorylation of ULK1 by AMPK regulates translocation of ULK1 to mitochondria and mitophagy, FEBS Lett. 589 (2015) 1847-1854. https://doi.org/10.1016/j.febslet.2015.05.020.

[21] L. Shang, S. Chen, F. Du, S. Li, L. Zhao, X. Wang, Nutrient starvation elicits an acute autophagic response mediated by Ulk1 dephosphorylation and its subsequent dissociation from AMPK, Proc. Natl. Acad. Sci. 108 (2011) 4788-4793. https://doi.org/10.1073/pnas.1100844108.

[22] D.G. McEwan, I. Dikic, The three musketeers of autophagy: Phosphorylation, ubiquitylation and acetylation, Trends Cell Biol. 21 (2011) 195-201. https://doi.org/10.1016/j.tcb.2010.12.006.

[23] T. Hanada, N.N. Noda, Y. Satomi, Y. Ichimura, Y. Fujioka, T. Takao, F. Inagaki, Y. Ohsumi, The Atg12-Atg5 conjugate has a novel E3-like activity for protein lipidation in autophagy, J. Biol. Chem. 282 (2007) 37298-37302. https://doi.org/10.1074/jbc.C700195200.

[24] R. Li, X. Yi, X. Wei, B. Huo, X. Guo, C. Cheng, Z.-M. Fang, J. Wang, X. Feng, P. Zheng, Y.-S. Su, J.F. Masau, X.-H. Zhu, D.-S. Jiang, EZH2 inhibits autophagic cell death of aortic vascular smooth muscle cells to affect aortic dissection, Cell Death Dis. 9 (2018) 180. https://doi.org/10.1038/s41419-017-0213-2.

[25] H.-J.R. Shin, H. Kim, S. Oh, J.-G. Lee, M. Kee, H.-J. Ko, M.-N. Kweon, K.-J. 
Won, S.H. Baek, AMPK-SKP2-CARM1 signalling cascade in transcriptional regulation of autophagy, Nature. 534 (2016) 553-557.

https://doi.org/10.1038/nature18014.

[26] R. Al-Attar, K.B. Storey, Suspended in time: Molecular responses to hibernation also promote longevity., Exp. Gerontol. 134 (2020) 110889. https://doi.org/10.1016/j.exger.2020.110889.

[27] L.J. Hawkins, K.B. Storey, Histone methylation in the freeze-tolerant wood frog (Rana sylvatica), J. Comp. Physiol. B. 188 (2018) 113-125. https://doi.org/10.1007/s00360-017-1112-7.

[28] J. Zhang, L.J. Hawkins, K.B. Storey, DNA methylation and regulation of DNA methyltransferases in a freeze-tolerant vertebrate, Biochem. Cell Biol. (2019) 1-9. https://doi.org/10.1139/bcb-2019-0091.

[29] S.N. Tessier, B.E. Luu, J.C. Smith, K.B. Storey, The role of global histone posttranslational modifications during mammalian hibernation, Cryobiology. 75 (2017) 28-36. https://doi.org/10.1016/j.cryobiol.2017.02.008.

[30] C.W. Wu, K.K. Biggar, J. Zhang, S.N. Tessier, F. Pifferi, M. Perret, K.B. Storey, Induction of antioxidant and heat shock protein responses during torpor in the Gray Mouse Lemur, Microcebus murinus, Genomics, Proteomics Bioinforma. 13 (2015) 19-126. https://doi.org/10.1016/j.gpb.2015.03.004.

[31] K. Storey, Storey, Heat shock proteins and hypometabolism: adaptive strategy for proteome preservation, Res. Rep. Biol. 2011 (2011) 57. https://doi.org/10.2147/RRB.S13351. 
[32] J.M. Storey, K.B. Storey, In defense of proteins: Chaperones respond to freezing, anoxia, or dehydration stress in tissues of freeze tolerant wood frogs, J. Exp. Zool. Part A Ecol. Integr. Physiol. 331 (2019) 392-402. https://doi.org/10.1002/jez.2306.

[33] C.-W. Wu, S.N. Tessier, K.B. Storey, Stress-induced antioxidant defense and protein chaperone response in the freeze-tolerant wood frog Rana sylvatica, Cell Stress Chaperones. 23 (2018) 1205-1217. https://doi.org/10.1007/s12192-0180926-x.

[34] D.R. Joanisse, K.B. Storey, Oxidative damage and antioxidants in Rana sylvatica, the freeze-tolerant wood frog., Am. J. Physiol. 271 (1996) R545-53. https://doi.org/10.1152/ajpregu.1996.271.3.R545.

[35] K.B. Storey, J.M. Storey, Metabolic rate depression in animals: Transcriptional and translational controls, Biol. Rev. 79 (2004) 207-233. https://doi.org/10.1017/S1464793103006195.

[36] Á. Bánréti, M. Sass, Y. Graba, The emerging role of acetylation in the regulation of autophagy, Autophagy. 9 (2013) 819-829. https://doi.org/10.4161/auto.23908.

[37] R. Al-Attar, S. Wijenayake, K.B. Storey, Metabolic reorganization in winter: Regulation of pyruvate dehydrogenase (PDH) during long-term freezing and anoxia., Cryobiology. 86 (2019) 10-18. https://doi.org/10.1016/j.cryobiol.2019.01.006.

[38] S. Zhao, L. Lin, G. Kan, C. Xu, Q. Tang, C. Yu, W. Sun, L. Cai, C. Xu, S. Cui, High autophagy in the naked mole rat may play a significant role in maintaining good health, Cell. Physiol. Biochem. 33 (2014) 321-332. 
https://doi.org/10.1159/000356672.

[39] T. Johnson, L. Zhao, G. Manuel, H. Taylor, D. Liu, Approaches to therapeutic angiogenesis for ischemic heart disease, J. Mol. Med. 97 (2019) 141-151. https://doi.org/10.1007/s00109-018-1729-3.

[40] M.G. Tonnesen, X. Feng, R.A.F. Clark, Angiogenesis in wound healing, J. Investig. Dermatology Symp. Proc. 5 (2000) 40-46. https://doi.org/10.1046/j.1087-0024.2000.00014.x.

[41] K.B. Storey, J.M. Storey, Biochemical adaption for freezing tolerance in the wood frog, Rana sylvatica, J. Comp. Physiol. B. 155 (1984) 29-36. https://doi.org/10.1007/BF00688788.

[42] J.A. MacDonald, T. Degenhardt, J.W. Baynes, K.B. Storey, Glycation of wood frog (Rana sylvatica) hemoglobin and blood proteins: In vivo and in vitro studies, Cryobiology. 59 (2009) 223-225. https://doi.org/10.1016/j.cryobiol.2009.06.008.

[43] S. Yang, L. Xu, T. Yang, F. Wang, High-mobility group box-1 and its role in angiogenesis, J. Leukoc. Biol. 95 (2014) 563-574. https://doi.org/10.1189/jlb.0713412.

[44] J. Evankovich, S.W. Cho, R. Zhang, J. Cardinal, R. Dhupar, L. Zhang, J.R. Klune, J. Zlotnicki, T. Billiar, A. Tsung, High mobility group box 1 release from hepatocytes during ischemia and reperfusion injury is mediated by decreased histone deacetylase activity., J. Biol. Chem. 285 (2010) 39888-97. https://doi.org/10.1074/jbc.M110.128348. 
[45] R. Kang, K.M. Livesey, H.J. Zeh, M.T. Lotze, D. Tang, HMGB1: A novel Beclin 1-binding protein active in autophagy, Autophagy. 6 (2010) 1209-1211. https://doi.org/10.4161/auto.6.8.13651.

[46] S. Gardella, C. Andrei, D. Ferrera, L. V. Lotti, M.R. Torrisi, M.E. Bianchi, A. Rubartelli, The nuclear protein HMGB1 is secreted by monocytes via a nonclassical, vesicle-mediated secretory pathway, EMBO Rep. 3 (2002) 995-1001. https://doi.org/10.1093/embo-reports/kvf198.

[47] Y. Xu, F. Toure, W. Qu, L. Lin, F. Song, X. Shen, R. Rosario, J. Garcia, A.M. Schmidt, S.F. Yan, Advanced glycation end product (AGE)-receptor for age (RAGE) signaling and up-regulation of Egr-1 in hypoxic macrophages, J. Biol. Chem. 285 (2010) 23233-23240. https://doi.org/10.1074/jbc.M110.117457.

[48] J.S. Chang, T. Wendt, W. Qu, L. Kong, Y.S. Zou, A.M. Schmidt, S.F. Yan, Oxygen deprivation triggers upregulation of early growth response-1 by the receptor for advanced glycation end products, Circ. Res. 102 (2008) 905-913. https://doi.org/10.1161/CIRCRESAHA.107.165308.

[49] A. Gupta, K.B. Storey, Regulation of antioxidant systems in response to anoxia and reoxygenation in Rana sylvatica, Comp. Biochem. Physiol. Part B Biochem. Mol. Biol. 243-244 (2020) 110436. https://doi.org/10.1016/j.cbpb.2020.110436.

[50] E.M. Ellis, Reactive carbonyls and oxidative stress: Potential for therapeutic intervention, Pharmacol. Ther. 115 (2007) 13-24. https://doi.org/10.1016/j.pharmthera.2007.03.015.

[51] N.P.J. Brindle, P. Saharinen, K. Alitalo, Signaling and functions of angiopoietin-1 
in vascular protection., Circ. Res. 98 (2006) 1014-23.

https://doi.org/10.1161/01.RES.0000218275.54089.12.

[52] T. Asahara, D. Chen, T. Takahashi, K. Fujikawa, M. Kearney, M. Magner, G.D. Yancopoulos, J.M. Isner, Tie2 Receptor Ligands, Angiopoietin-1 and Angiopoietin-2, Modulate VEGF-Induced Postnatal Neovascularization, Circ. Res. 83 (1998) 233-240. https://doi.org/10.1161/01.RES.83.3.233.

[53] S. Davis, T.H. Aldrich, P.F. Jones, A. Acheson, D.L. Compton, V. Jain, T.E. Ryan, J. Bruno, C. Radziejewski, P.C. Maisonpierre, G.D. Yancopoulos, Isolation of angiopoietin-1, a ligand for the TIE2 receptor, by secretion-trap expression cloning, Cell. 87 (1996) 1161-1169. https://doi.org/10.1016/S00928674(00)81812-7.

[54] M. Shiota, H. Izumi, N. Miyamoto, T. Onitsuka, E. Kashiwagi, A. Kidani, G. Hirano, M. Takahashi, M. Ono, M. Kuwano, S. Naito, Y. Sasaguri, K. Kohno, Ets regulates peroxiredoxin 1 and 5 expressions through their interaction with the highmobility group protein B1, Cancer Sci. 99 (2008) 1950-1959. https://doi.org/10.1111/j.1349-7006.2008.00912.x.

[55] M.L. Verschoor, L.A. Wilson, C.P. Verschoor, G. Singh, Ets-1 Regulates Energy Metabolism in Cancer Cells, PLoS One. 5 (2010) e13565. https://doi.org/10.1371/journal.pone.0013565.

[56] M.L. Verschoor, C.P. Verschoor, G. Singh, Ets-1 global gene expression profile reveals associations with metabolism and oxidative stress in ovarian and breast cancers, Cancer Metab. 1 (2013) 17. https://doi.org/10.1186/2049-3002-1-17. 
[57] X. Zhang, D. Wu, M. Aldarouish, X. Yin, C. Li, C. Wang, ETS-1: A potential target of glycolysis for metabolic therapy by regulating glucose metabolism in pancreatic cancer, Int. J. Oncol. 50 (2017) 232-240. https://doi.org/10.3892/ijo.2016.3770.

[58] G. Mavrothalassitis, J. Ghysdael, Proteins of the ETS family with transcriptional repressor activity, Oncogene. 19 (2000) 6524-6532. https://doi.org/10.1038/sj.onc.1204045.

[59] D. Goldman, M.K. Sapru, S. Stewart, J. Plotkin, T.A. Libermann, B. Wasylyk, K. Guan, Cloning and characterization of GETS-1, a goldfish Ets family member that functions as a transcriptional repressor in muscle, Biochem. J. 335 (1998) 267275. https://doi.org/10.1042/bj3350267.

[60] J. St-Pierre, M.D. Brand, R.G. Boutilier, Mitochondria as ATP consumers: Cellular treason in anoxia, Proc. Natl. Acad. Sci. 97 (2000) 8670-8674. https://doi.org/10.1073/pnas.140093597.

[61] M.E. Pamenter, C.R. Gomez, J.G. Richards, W.K. Milsom, Mitochondrial responses to prolonged anoxia in brain of red-eared slider turtles, Biol. Lett. 12 (2016) 20150797. https://doi.org/10.1098/rsbl.2015.0797.

[62] T.K. Kundu, M. Velayutham, J.L. Zweier, Aldehyde oxidase functions as a superoxide generating NADH oxidase: An important redox regulated pathway of cellular oxygen radical formation, Biochemistry. 51 (2012) 2930-2939. https://doi.org/10.1021/bi3000879.

[63] M.P. Hoyeck, H. Hadj-Moussa, K.B. Storey, The role of MEF2 transcription 
factors in dehydration and anoxia survival in Rana sylvatica skeletal muscle, PeerJ. 5 (2017) e4014. https://doi.org/10.7717/peerj.4014.

[64] E.-L. Eskelinen, F. Reggiori, M. Baba, A.L. Kovács, P.O. Seglen, Seeing is believing: The impact of electron microscopy on autophagy research, Autophagy. 7 (2011) 935-956. https://doi.org/10.4161/auto.7.9.15760.

[65] R. Mundade, H.G. Ozer, H. Wei, L. Prabhu, T. Lu, Role of ChIP-seq in the discovery of transcription factor binding sites, differential gene regulation mechanism, epigenetic marks and beyond, Cell Cycle. 13 (2014) 2847-2852. https://doi.org/10.4161/15384101.2014.949201.

[66] B. Kaboord, M. Perr, Isolation of proteins and protein complexes by immunoprecipitation, in: Methods Mol. Biol., 2008: pp. 349-364. https://doi.org/10.1007/978-1-60327-064-9_27. 


\section{Appendix A: List of current and future publications}




\section{$\underline{\text { Manuscript published in peer reviewed journals }}$}

1. R. Al-attar, Y. Zhang, K.B. Storey, Osmolyte regulation by TonEBP/NFAT5 during anoxia-recovery and dehydration-rehydration stresses in the freeze-tolerant wood frog (Rana sylvatica), PeerJ. 5 (2017) e2797. doi:10.7717/peerj.2797.

2. Y. Zhang, R. Al-attar, K.B. Storey, TonEBP/NFAT5 regulates downstream osmoregulatory proteins during freeze-thaw stress in the wood frog, Cryobiology. 79 (2017) 43-49. doi:10.1016/j.cryobiol.2017.09.003.

3. L.J. Hawkins*, R. Al-attar*, K.B. Storey, Transcriptional regulation of metabolism in disease: From transcription factors to epigenetics, PeerJ. 6 (2018) e5062. doi:10.7717/peerj.5062. (*equal contribution)

4. R. Al-Attar, K.B. Storey, Effects of anoxic exposure on the nuclear factor of activated T cell (NFAT) transcription factors in the stress-tolerant wood frog., Cell Biochem. Funct. $\quad 36$ (2018) 420-430. doi:10.1002/cbf.3362.

5. R. Al-Attar*, S. Wijenayake*, K.B. Storey, Metabolic reorganization in winter: regulation of pyruvate dehydrogenase $(\mathrm{PDH})$ during long-term freezing and anoxia., Cryobiology. 86 (2019) 10-18. doi:10.1016/j.cryobiol.2019.01.006. (*equal contribution)

6. J.-Y. Zhang, B.E. Luu, D.-N. Yu, L.-P. Zhang, R. Al-attar, K.B. Storey, The complete mitochondrial genome of Dryophytes versicolor: Phylogenetic relationship among Hylidae and mitochondrial protein-coding gene expression in response to freezing and anoxia, Int. J. Biol. Macromol. 132 (2019) 461-469. doi:10.1016/j.ijbiomac.2019.03.220.

7. R. Al-attar*, C.-W. Wu*, K.K. Biggar, K. Storey, Carb-loading: Freeze-induced activation of the glucose-responsive ChREBP transcriptional network in wood frogs, Physiol. Biochem. Zool. (2019) 706463. https://doi.org/10.1086/706463. (* equal contribution)

8. R. Al-attar, C.L. Childers, V.C. Nguyen, M.E. Pamenter, K.B. Storey, Differential protein phosphorylation is responsible for hypoxia-induced regulation of the Akt/mTOR pathway in naked mole rats, Comp. Biochem. Physiol. Part A Mol. Integr. Physiol. 242 (2020) 110653. https://doi.org/10.1016/j.cbpa.2020.110653. (Special issue)

9. R. Al-Attar, K.B. Storey, Suspended in time: Molecular responses to hibernation also promote longevity., Exp. Gerontol. 134 (2020) 110889.

https://doi.org/10.1016/j.exger.2020.110889.(Special issue, invited review)

10. R. Al-Attar, K.B. Storey, RAGE against the stress: Mitochondrial suppression in hypometabolic hearts., $\quad$ Gene. $\quad 761 \quad$ (2020) 145039. https://doi.org/10.1016/j.gene.2020.145039.

11. S.R. Green, R. Al-Attar, A.E. McKechnie, S. Naidoo, K.B. Storey, Role of Akt signaling pathway regulation in the speckled mousebird (Colius striatus) during torpor displays tissue specific responses, Cell. Signal. 75 (2020) 109763.

https://doi.org/10.1016/j.cellsig.2020.109763. 
Manuscripts currently under review in peer-reviewed journals

1. R. Saleem, R. Al-attar, K.B. Storey, Activation of pro-survival pathways of the north American Myotis lucifugus, during hibernation, Cryobiology (Cryo_2020_16, in revision)

Non-manuscript peer-reviewed contributions

1. R. Al-attar, K. Storey, Surviving winter: NFATs regulate cryoprotection in freezetolerant Rana sylvatica, Cryobiology. 73 (2016) 430. https://doi.org/10.1016/j.cryobiol.2016.09.120.

2. S. Wijenayake, R. Al-attar, S. Tessier, K. Storey, Hibernation vs freezing: The tale of metabolic reorganization in winter, Cryobiology. 73 (2016) 427. https://doi.org/10.1016/j.cryobiol.2016.09.111.

3. R. Al-attar, K. B. Storey, Organ Cryopreservation: Nature's Protocol For Freezing Organs, Sci. Trends. (2018). https://doi.org/10.31988/SciTrends.26890.

\section{Commentary written about my work}

1. O. Birceanu, Recovering from oxygen deprivation, froggy style!, J. Exp. Biol. 222 (2019) jeb192658. https://doi.org/10.1242/jeb.192658

\section{Completed projects (not submitted)}

\section{0}

1. R. Al-attar, K. B. Storey, Multifactorial regulation of autophagy during anoxia and dehydration in stress-tolerant wood frogs- Data collection complete

2. R. Al-attar, O. Aguilar, K. B. Storey, Regulation of autophagy during freezing in wood frogs- Data collection complete

3. Gupta, R. Al-attar, K.B. Storey, Regulation of apoptosis and antioxidant response during hypoxia in jumbo squids- Data collection complete

4. R. Al-attar*, L. J. Hawkins*, N. Bedard, S. S. Wing, K. B. Storey, Differential regulation of microRNAs in the liver of USP19 null mice after dexamethasone injection- Data collection complete (*equal contribution)2021

\section{1}

5. S. English, R. Al-attar, K.B. Storey, NFAT5-mediated osmoregulation in dehydration tolerant Xenopus laevis- Data collection complete

6. S. Mahrous, J. Robert, R. Al-attar, K.B. Storey, GATA4-mediated transcriptional network in skeletal and cardiac muscle of wood frogs- Data collection complete

\section{2}

7. R. Al-attar, K. B. Storey, RAGE management: Cyto-nuclear cross talk under oxidative stress- Data collection complete

8. R. Al-attar, K. B. Storey, Involvement of RAGE signaling in angiogenesis- Data collection complete

9. Y. Zhang, R. Al-attar, K. B. Storey, NFAT signaling during dehydration in wood 
frogs- Data collection complete

10. J. Robert, R. Al-attar, K.B. Storey, Anti-apoptotic responses during freezing and dehydration in wood frogs- Data collection complete

11. S. Wijenayake, R. Al-attar, K.B. Storey, Post-translational modification of the pyruvate dehydrogenase complex in hyperglycemic conditions- Data collection complete

2023

12. R. Al-attar, S. Green, A. E. McKechnie, S. Naidoo, K. B. Storey, Involvement of MAPK signaling in regulating apoptosis in torpid speckled mousebirds- Data collection complete

13. S. Green, R. Al-attar, A. E. McKechnie, S. Naidoo, K. B. Storey, Post-translational regulation of the pyruvate dehydrogenase complex during torpor in speckled mousebirds- Data collection complete

\section{4}

14. R. Al-attar*, A.J. Watts*, K. B. Storey, Involvement of microRNA in regulating anoxia-tolerance in tree frogs- Data collection complete (*equal contribution)

15. S. Mahrous, R. Al-attar, K. B. Storey, Regulation of the antioxidant response in gray tree frogs- Data collection complete

16. R. Al-attar, K. B. Storey, Regulation of the NFAT transcriptional response during freezing in wood frogs- Data collection complete 
Appendix B: List of Scientific communications at meetings 
1. Al-attar, R., K. B. Storey, "Multifaceted regulation of autophagy and lysosomal biogenesis supports environmental stress-tolerance in wood frogs". The $10^{\text {th }}$ International Congress of Comparative Physiology and Biochemistry, Ottawa, Ontario, Canada, August 5-9, 2019 (Oral presentation).

2. R. Al-attar, K. B. Storey, "RAGE management: Cyto-nuclear cross talk under oxidative stress". The $10^{\text {th }}$ International Congress of Comparative Physiology and Biochemistry, Ottawa, Ontario, Canada, August 5-9, 2019 (Oral presentation).

3. R. Al-attar, S. Mahrous, J. Robert, K. B. Storey, "GATA4-mediated gene expression promotes muscle remodeling during stress in the freeze-tolerant wood frog, Rana sylvatica". The $21^{\text {st }}$ annual Chemistry and Biochemistry Graduate Research Conference, Montreal, Quebec, Canada, Nov 9 ${ }^{\text {th }}$, 2018. (Poster presentation)

4. R. Al-attar, K. B. Storey, Regulation of autophagy-related proteins in the freezetolerant wood frog, Rana sylvatica. $13^{\text {th }}$ annual meeting of the International Conference on Genomics (ICG-13), Shenzhen, China, Oct 24-28, 2018. (Poster presentation- Recipient of the Outstanding Poster Award)

5. R. Al Al-attar, L. J. Hawkins, N. Bedard, S. S. Wing, K. B. Storey, Differential regulation of microRNAs in the liver of USP10 null mice during fasting. $2^{\text {nd }}$ annual Toronto RNA Enthusiasts Day symposium, Toronto, Ontario, Canada, August 2, 2017. (Poster presentation)

6. R. Al-attar, K. B. Storey, Surviving anoxia: Regulation of the autophagyrelated proteins in the freeze-tolerant wood frog, Rana sylvatica. $60^{\text {th }}$ Annual national meeting of the Canadian Society for Molecular Biosciences conference, Ottawa, Ontario, Canada, May 16-21, 2017. (Poster presentation)

7. R. Al-attar, K. B. Storey, Surviving winter: NFATs regulate cryoprotection in freeze-tolerant Rana sylvatica. $53^{\text {rd }}$ Annual international meeting of the Society for Cryobiology, Ottawa, Ontario, Canada, July 23-27, 2016. (Oral presentation, Invited speaker)- Cryobiology 73, 430 (2016)

8. S. Wijenayake, R. Al-attar, K. B. Storey, Hibernation vs Freezing: The Tale of Metabolic Reorganization in Winter. $53^{\text {rd }}$ Annual Meeting of the Society for Cryobiology, Ottawa, Ontario, Canada, July 23-27, 2016. (Oral presentation)Cryobiology 73, 427 (2016)

9. R. Al-attar, K. B. Storey, Surviving diabetes: The tale of NFATs and the frozen frog. Ottawa-Carleton Institute of Biology Symposium, Ottawa, University, Ottawa, Ontario, Canada, April 5, 2016. ( $3^{\text {rd }}$ prize winter for oral presentations).

10. R. Al-attar, K. B. Storey, The Role of NFATs in Molecular Adaptation to Stress in Wood Frogs. Ottawa-Carleton Student Northern Research Symposium 2015, Carleton University, Ottawa, Ontario, Canada, March 6, 2015. ( $\mathbf{1}^{\text {st }}$ prize-poster presentations).

11. R. Al-attar, S. Wijenayake, K. B. Storey, Pyruvate Dehydrogenase Kinase (1-4) Regulation under Freezing, Anoxia and Dehydration in Liver \& Muscle of Rana Sylvatica. $12^{\text {th }}$ Annual Ottawa-Carleton Institute of Biology Symposium, Carleton University, Ottawa, Ontario, Canada, April 29, 2015. (Poster presentation) 
Appendix C: List of
antibodies and immunoblot
conditions 
Table C1: Immunoblot antibody and protein information.

\begin{tabular}{|l|l|l|l|}
\hline Target & $\begin{array}{l}\text { Molecular } \\
\text { weight (kDa) }\end{array}$ & Gel (\%) & $\begin{array}{l}\text { Secondary } \\
\text { antibody } \\
\text { (1:5000) }\end{array}$ \\
\hline ULK1 & 120 & 8 & Anti-rabbit \\
\hline $\begin{array}{l}\text { p-ULK1 } \\
\text { (S555) }\end{array}$ & 124 & 8 & Anti-rabbit \\
\hline $\begin{array}{l}\text { p-ULK1 } \\
\text { S758 }\end{array}$ & 121 & 8 & Anti-rabbit \\
\hline ATG4a & 45 & 12 & Anti-rabbit \\
\hline TFEB & 49 & 12 & Anti-rabbit \\
\hline ATG14 & 58 & 10 & Anti-rabbit \\
\hline LC3B & 15 & 15 & Anti-rabbit \\
\hline ATPV6VD1 & 45 & 12 & Anti-rabbit \\
\hline ATG3 & 36 & 15 & Anti-rabbit \\
\hline ATG5L & 36 & 15 & Anti-rabbit \\
\hline Beclin1 & 56 & 12 & Anti-rabbit \\
\hline $\begin{array}{l}\text { p-Beclin1 } \\
\text { (S93) }\end{array}$ & 58 & 12 & Anti-rabbit \\
\hline p62 & 50 & 12 & Anti-rabbit \\
\hline $\begin{array}{l}\text { Ac-HMGb1 } \\
\text { (K29) }\end{array}$ & 29 & 15 & Anti-rabbit \\
\hline NAB1 & 51 & 15 & Anti-rabbit \\
\hline NAB2 & 53 & 15 & Anti-rabbit \\
\hline FGF2 & 17 & 15 & Anti-rabbit \\
\hline ANGPT1 & 58 & 10 & Anti-rabbit \\
\hline ANGPT2 & 57 & 10 & Anti-rabbit \\
\hline Tie1 & 125 & 8 & Anti-rabbit \\
\hline Tie2 & 127 & 8 & Anti-rabbit \\
\hline VEGFR1 & 77 & 8 & Anti-rabbit \\
\hline Ve-cad & 90 & 8 & Anti-rabbit \\
\hline ETS1 & 50 & 15 & Anti-rabbit \\
\hline EGR1 & 54 & 12 & Anti-rabbit \\
\hline RAGE & 42 & 15 & Anti-rabbit \\
\hline VEGF & 35 & 15 & Anti-rabbit \\
\hline HMGB1 & 26 & 15 & Anti-mouse \\
\hline PKCII 3660 & 76 & 12 & Anti-rabbit \\
\hline AOX1 & 145 & 6 & Anti-rabbit \\
\hline CYC1 & 34 & 15 & Anti-rabbit \\
\hline p-ETS1 T38 & 51 & 15 & Anti-rabbit \\
\hline GLUT1 & 54 & 12 & Anti-rabbit \\
\hline PRX1 & 22 & 15 & Anti-rabbit \\
\hline PRX5 & 27 & 15 & Anti-rabbit \\
\hline ERK2 & 42 & 15 & Anti-mouse \\
\hline ATP5A1 & 59 & 10 & Anti-rabbit \\
\hline & & & \\
\hline
\end{tabular}




\begin{tabular}{|l|l|l|l|}
\hline NDUFAB1 & 17 & 15 & Anti-rabbit \\
\hline CS & 51.8 & 12 & Anti-rabbit \\
\hline
\end{tabular}




\section{Appendix D: Representative blots}


A

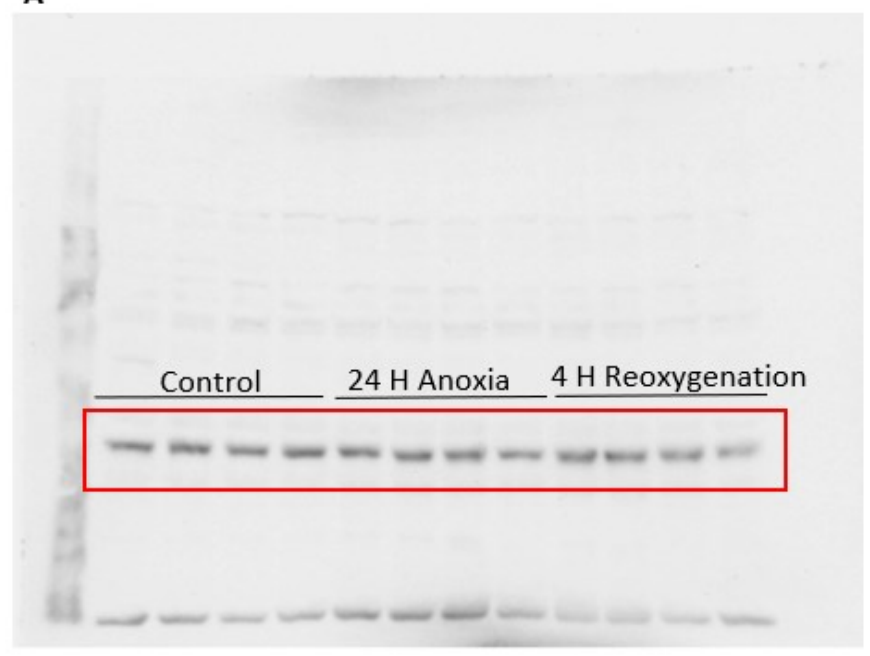

B

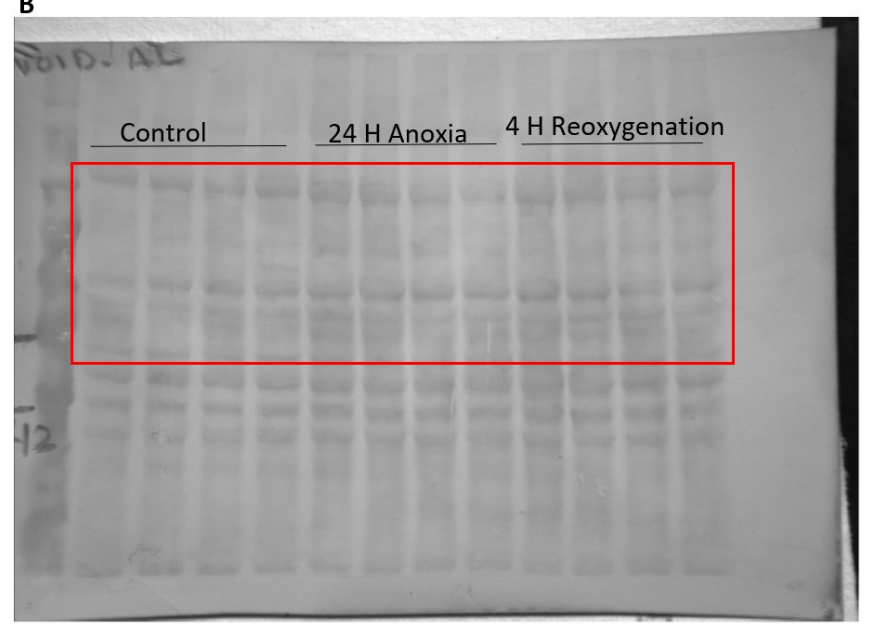

Figure D1: Representative immunoblot showing the A) enhanced chemiluminescent band and B) Coomassie stained membrane of the ATP6V0D1 protein in liver of frogs exposed to control, 24-hour anoxic, and 4-hour reoxygenated treatments. Red boxes represent the bands quantified. 


\section{Appendix E: Standard curve for AGE-BSA}




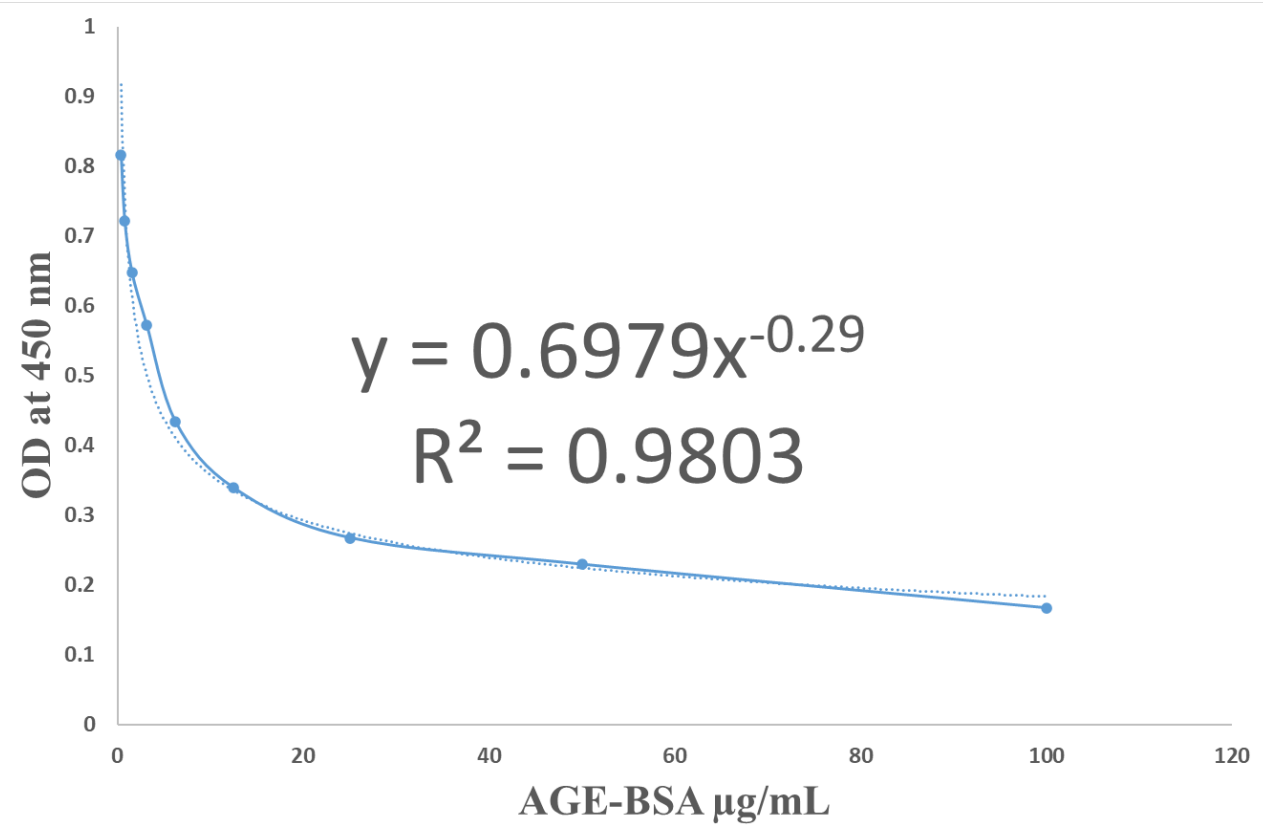

Figure E1: Standard curve generated using AGE-BSA standard following the manufacturer's protocol. 
Appendix F: Extra data 
Due to abrupt closure of laboratories because of COVID-19, the fourth data chapter is incomplete. Missing measurements are targets of early apoptosis markers (measured using the MILLIPLEX MAP Early Apoptosis Magnetic Bead 7-plex Kit - Cell Signaling Multiplex Assay (MilliporeSigma, cat no. 48-669MAG). This kit has been purchased previously and will be used by Aakriti Gupta upon laboratory reopening at her convenience. Aakriti will receive second authorship for her contribution.

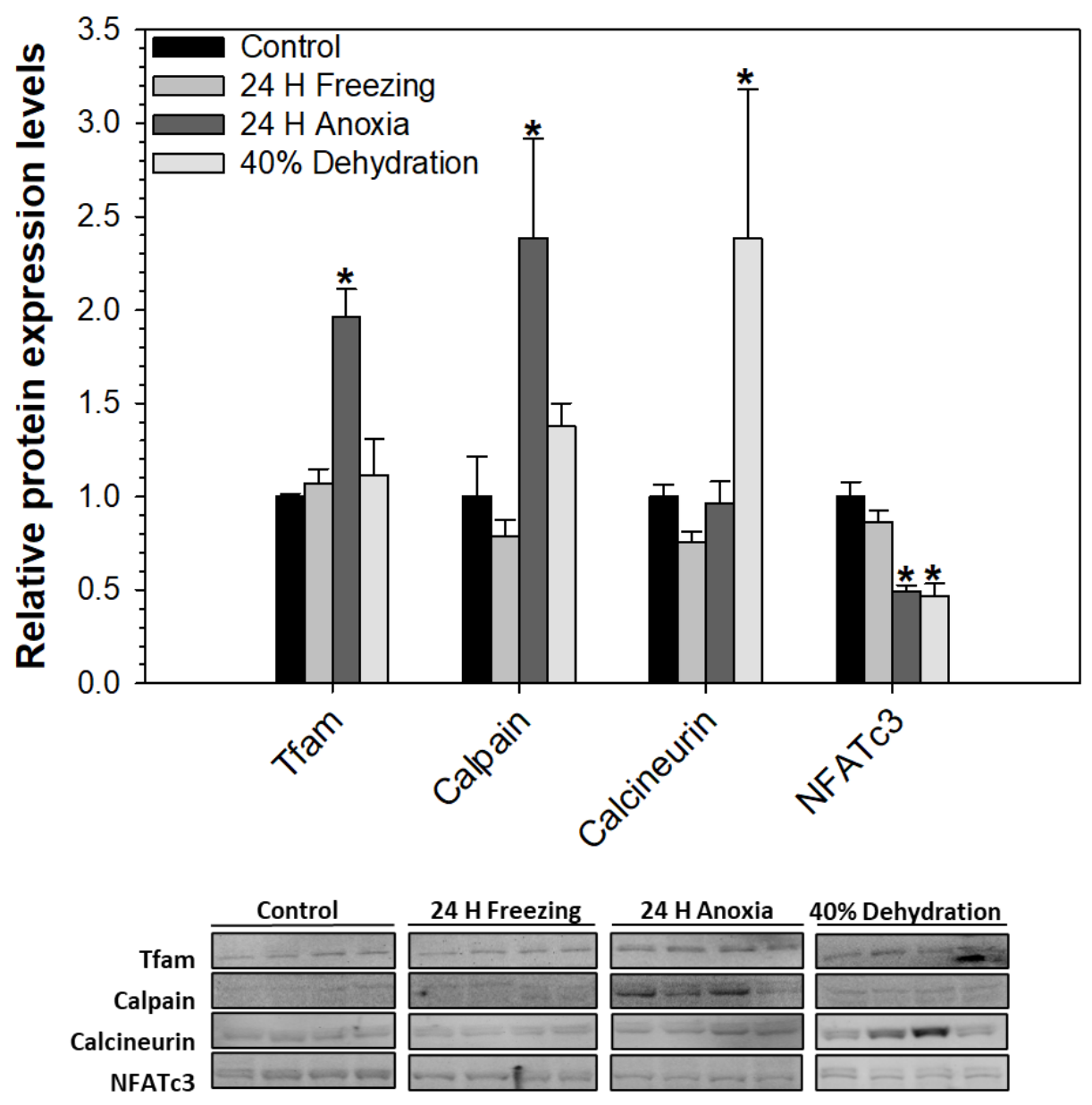

Figure F1: Analysis of upstream regulations of NFATc3 measured in wood frog heart in response to freezing, anoxia or dehydration measured by immunoblotting. Histogram shows mean \pm SEM of $n=4$ independent biological replicates from different frogs. Statistical significance from the control is denoted by $(*)(\mathrm{P}<0.05)$ and was calculated using a one-way ANOVA with a Dunnett post-hoc test. 

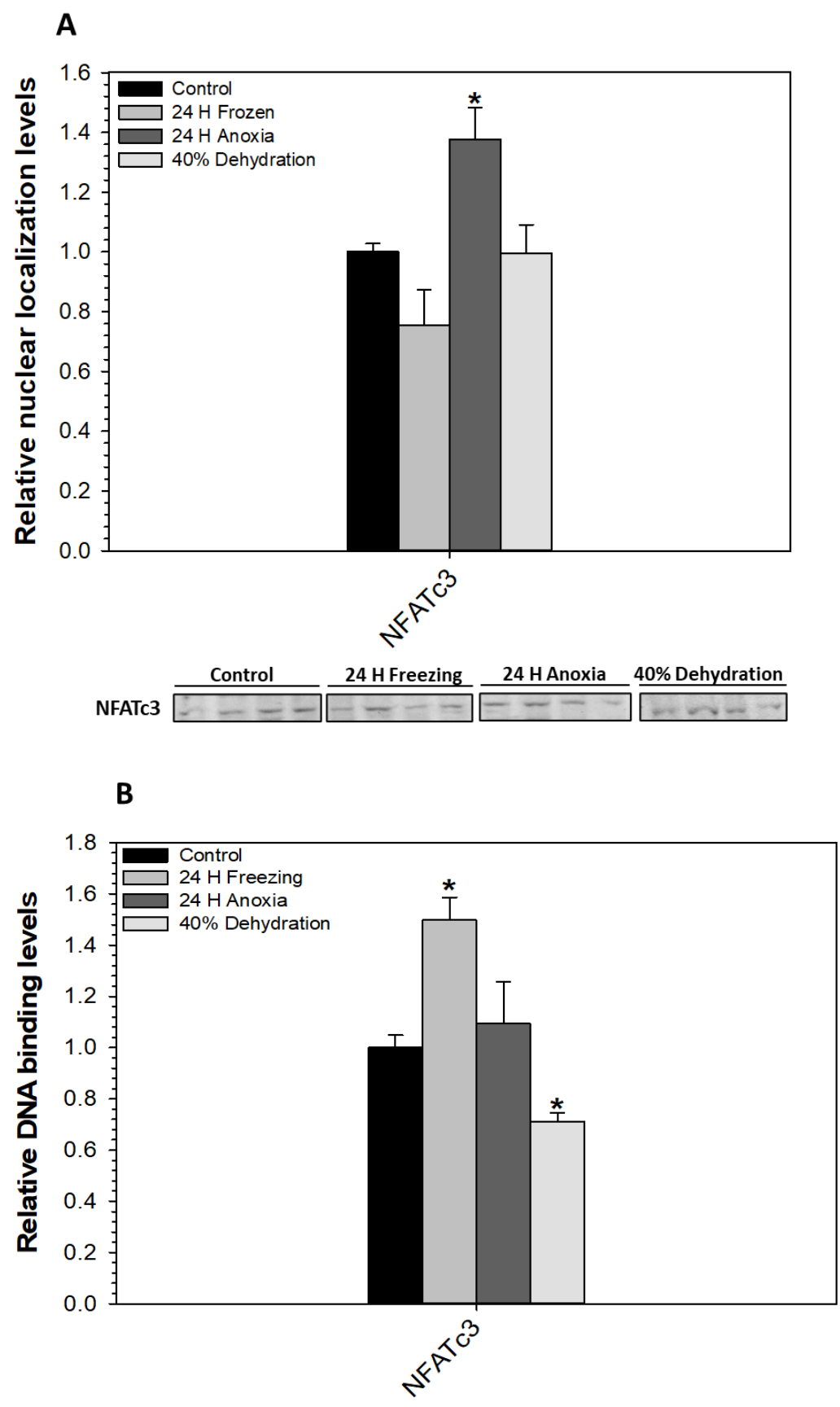

Figure F2: Relative nuclear levels of NFATc3 (A), and B) the DNA binding activity of total NFATc3 in heart of wood frogs exposed to freezing, anoxia and dehydration measured by immunoblotting. Please see Figure 1 for more information. 

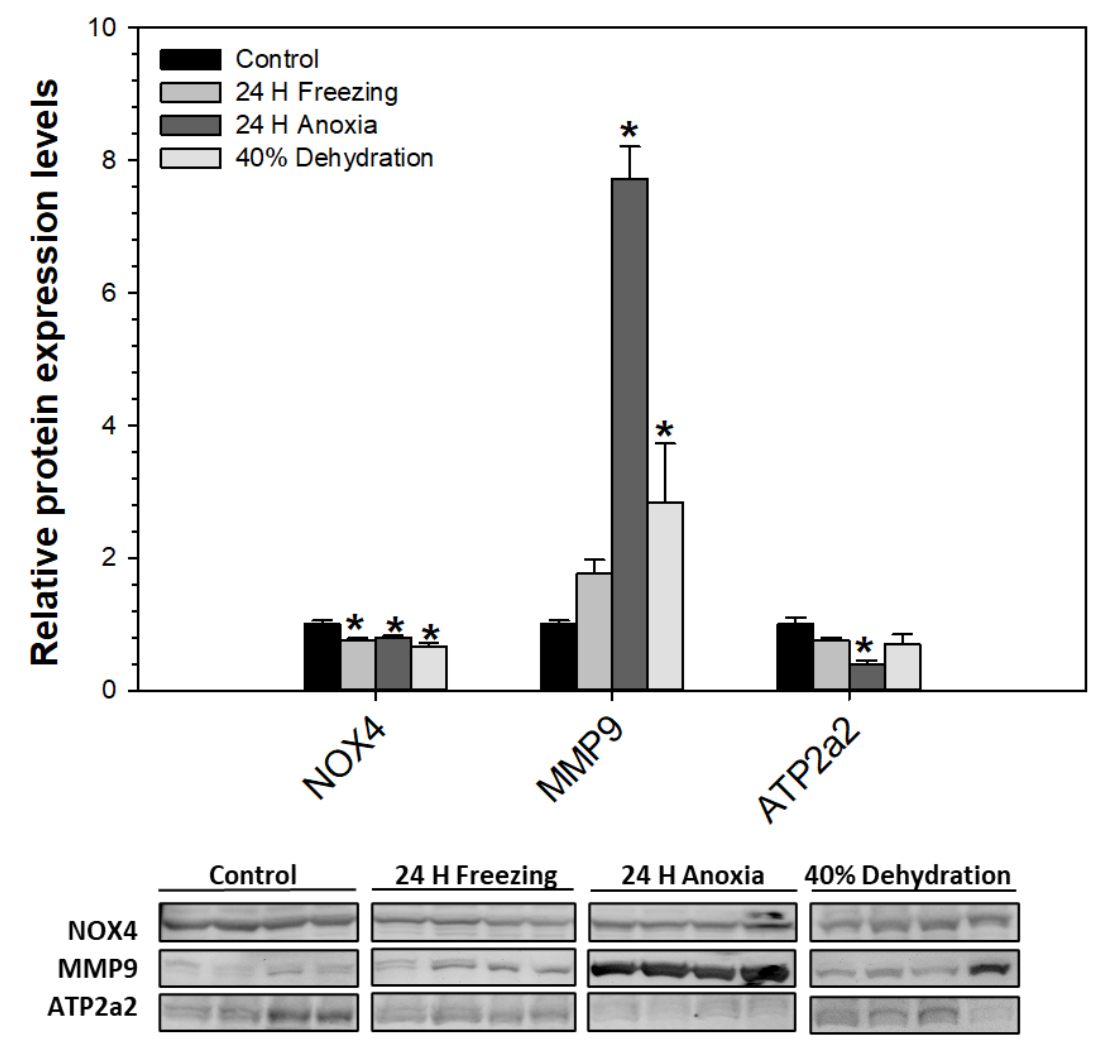

Figure F3: Relative protein levels of downstream targets of NFATc3 and Tfam in wood frog heart exposed to freezing, anoxia and dehydration measured by immunoblotting. Please see Figure 1 for more information. 

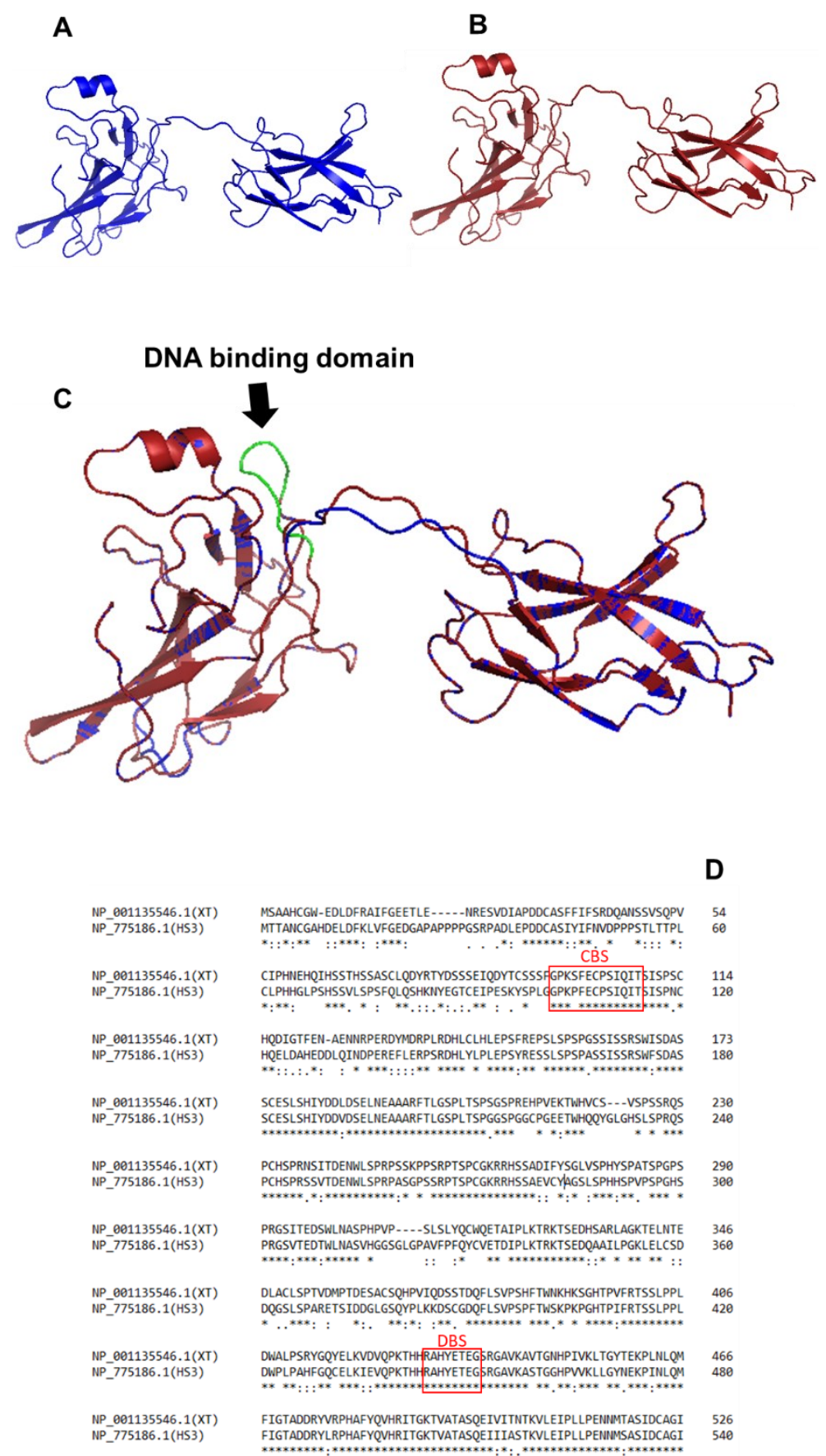

Figure F4: Schematic diagram showing the similarities between the partial NFATc3 protein sequence of Xenopus tropicalis (NP_001135546.1) and splice variant 3 of humans (N P_775186.1). Using the online Phyre2 program, the NFATc3 structure for Xenopus laevis (A) and the splice variant 3 of humans (B) were predicted. Using Pymol, both figures were superimposed $(\mathrm{C})$ to show conservancy between the two sequences. Using Clustal Omega, the protein sequence of NFATc3 from both organisms was aliened (D), and the Calcineurin binding site (CBS) and DNA binding Site (DBS) were annotated based on information retreated from literature. 
That's a wrap! And what an amazing/memorable journey it's been!

Thank you, Ken, Jan, Dr. DeRosa, Dr. Pamenter, and my examiners for reading my thesis (3)

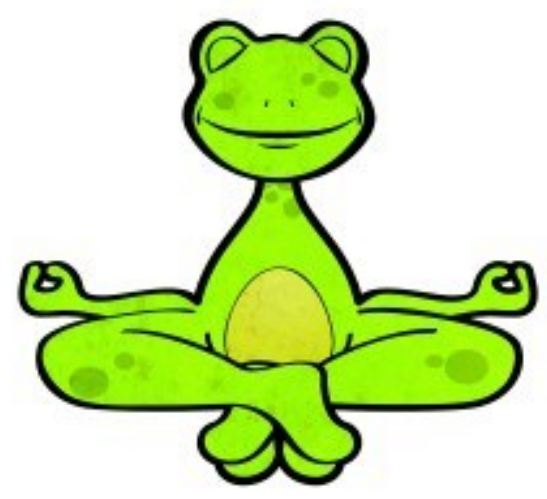

